\title{
Propositions
}

1. A single electric trigger always has multiple effects. (this thesis)

2. Electrostatic and hydration repulsion are key for electrochemical desorption of proteins. (this thesis)

3. Optimal optimization is for optimists.

4. Even though we all see the same data, we may not see the same picture.

5. Our brain is predisposed with prejudices and discrimination but our intellect lets us go beyond.

6. Gender equality can only be reached if we stop defining ourselves and others by gender.

Propositions belonging to this thesis, entitled

Electrochemical separation: From ions to proteins

Pina Atalanta Fritz

Wageningen, 9. August 2019 


\section{ELECTROCHEMICAL SEPARATION: FROM IONS TO PROTEINS}




\section{Thesis committee}

\section{Promotors}

Prof. Dr Karin Schroën

Personal chair at the Laboratory of Food Process Engineering

Wageningen University \& Research

Prof. Dr Remko Boom

Professor of Food Process Engineering

Wageningen University \& Research

\section{Co-promotor}

Prof. Dr Mary B. Chan-Park

Professor at the School of Chemical and Biomedical Engineering Nanyang Technological University, Singapore

\section{Other members}

Prof. Dr Albert van der Wal, Wageningen University \& Research

Prof. Dr Xu Rong, Nanyang Technological University, Singapore

Dr Sissi de Beer, University of Twente, The Netherlands

Dr Luc Scheres, Surfix BV, Wageningen, The Netherlands

This thesis was conducted under the auspices of the Graduate School SCBE (School of Chemical and Biomedical Engineering) at Nanyang Technological University, Singapore, and the Graduate School VLAG (Advanced studies in Food Technology, Agrobiotechnology, Nutrition and Health Science) at Wageningen University, The Netherlands, as part of the NTU-WU Joint PhD Program. 


\title{
ELECTROCHEMICAL SEPARATION: FROM IONS TO PROTEINS
}

\author{
Pina Atalanta Fritz
}

Thesis

submitted in fulfilment of the requirements for the joint degree of doctor between

Nanyang Technological University

by the authority of the Rector Magnificus, Prof. Dr Wang Xin

and Wageningen University

by the authority of the Rector Magnificus, Prof. Dr A. P. J. Mol,

in the presence of the

Thesis Committee appointed by the Academic Boards of both universities

to be defended in public

on Friday, December 13th 2019

at 11 a.m. in the Aula of Wageningen University 


\section{Pina Atalanta Fritz}

Electrochemical separation: From ions to proteins 216 pages

Joint PhD thesis,

Nanyang Technological University, Singapore,

and Wageningen University, Wageningen, the Netherlands (2019).

With references, with summary in English.

ISBN: 978-94-6395-127-2

DOI: https://doi.org/10.18174/500781 


\section{CONTENT}

\section{FIELD OF RESEARCH}

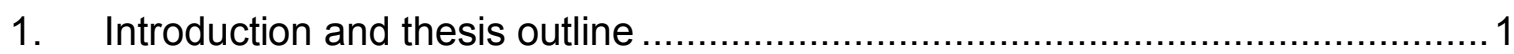

2. Electrochemically driven adsorptive separation techniques: From ions to proteins and cells in liquid streams .................................................... 7

\section{ION SEPARATION}

3. Exergy analysis of membrane capacitive deionization (MCDI)

4. Polyelectrolyte-activated carbon composite electrodes for inverted membrane capacitive deionization (iMCDI)

5. Zwitterionic polymer modified porous carbon for high performance and antifouling capacitive desalination

\section{PROTEIN SEPARATION}

6. Steering protein and salt adsorption and desorption by an electrical switch applied to tailor made electrodes

7. Controlling electrostatic and hydration interactions for protein adsorption and desorption

8. Simultaneous silicon oxide growth and electrophoretic deposition of graphene oxide.

\section{FINDINGS IN PERSPECTIVE}

9. General discussion 163

\section{MISCELANEOUS}

References

Summary

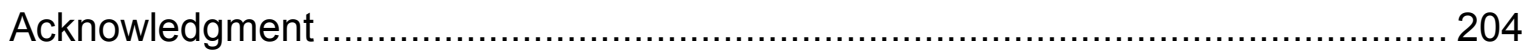

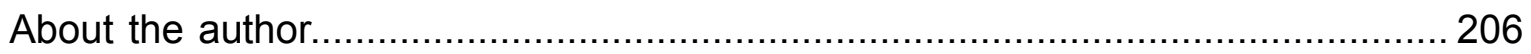

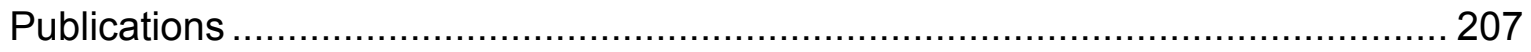

Training activities, list of conferences and teaching ...................................... 208 



\section{CHAPTER 1}

INTRODUCTION

AND

THESIS OUTLINE 


\section{INTRODUCTION AND THESIS OUTLINE}

Separations are at the heart of creating and producing new products. Before new products can be made, constituents need to be either isolated or fractionated from mixtures, and undesirable impurities need to be removed. For example, before we can enjoy a scoop of strawberry ice cream, milk needs to be centrifuged and filtered to obtain the cream (Tamime, 2008), sugar needs to be separated from the sugar beet juice and crystallized (Jenkins, 1966), and leaves need to be removed from strawberries. All these steps refer to specific separation techniques based on size, charge, color, solubility, etc.

In general, separation can be defined as a process to obtain at least two distinct products from a mixture depending on their physical or chemical specifications (Yoon et al., 2019). Five separation principles can be distinguished: the addition, or creation of an extra phase, as well as, the application of a barrier, a solid agent, or an external field (Figure 1.1). The first category (1) includes liquid-liquid extraction and stripping and the separation is based on the difference in miscibility of the molecules in the original phase and the newly added one. A new phase may be created by heat transfer to or from a mixture as is the case in distillation or crystallization (2). An example of a barrier is a membrane that allows the permeation of some components, while other components are retained (3). This can be done using porous or non-porous membranes, which can be used to separate colloidal particles or molecules. The underlying principle for typical components present in foods is size exclusion (e.g. proteins) or specific solubility and diffusivity in the membrane material (e.g. flavor molecules). Selective separation can also be achieved by interactions with inert or reactive solid agents as used in chromatography (4), or by applying an external force and/or gradient (5; e.g. pressure, electric field, magnetic field) as in centrifugation, electrophoresis, and electrodialysis (Seader et al., 1998).

All separation processes cost considerable energy, and are on large scale still dependent on the use of classic non-renewable energy sources such as natural oil and gas. Currently there is a strong drive toward the use of renewable energy sources, and it may be expected that the energy source of the future is electricity, generated through a mix of bio-based, solar, wind, tidal, etc. techniques. Thus, any separation 
technique that would inherently be compatible with this may hold advantages over others.

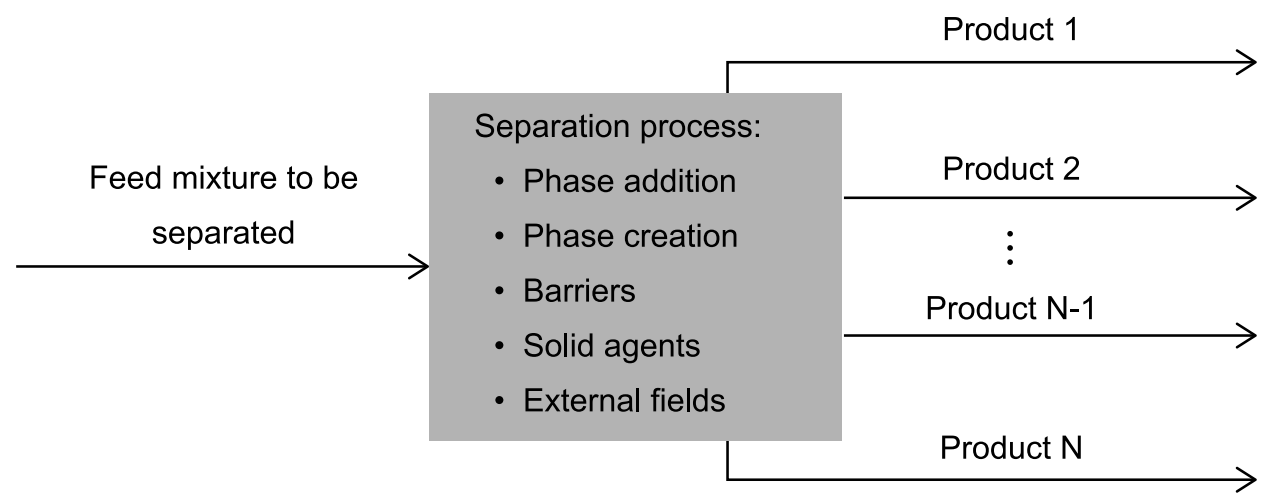

Figure 1.1. General schematic of separation processes and most common separation principles (Seader et al., 1998).

Away from the energy sourcing perspective, electric stimuli are also interesting in separation processes because their facile control enables quick cycling between the accumulation and regeneration stages. With a 'push of a button' the surface charge of an electrode can be changed from positive to negative or vice versa and thus influence the adsorption directly and quickly (Suss et al., 2015). In comparison to adsorptive separation processes that do not use electric driving forces (e.g. chromatography) the application of an electric stimulus avoids the use of solvents otherwise required to regenerate the solid agent: thus the electric potential replaces the chemical potential difference to desorb the target components.

Advanced processes often use a combination of separation principles; e.g., electrochemical ion separation processes such as capacitive deionization (CDI) make use of an external electric field, combined with a solid agent (the electrode) and often a barrier (ion exchange membranes). The electric field drives the ions towards the electrodes and accumulates them in the electric double layer when a constant potential or current is applied (Figure 1.2, left panels). The ions are released by reversing or switching off the potential or current. To make the process more efficient, ion exchange membranes are used to avoid co-ion expulsion (Suss et al., 2015; Yoon et al., 2019). 
Capacitive deionization

(CDI)
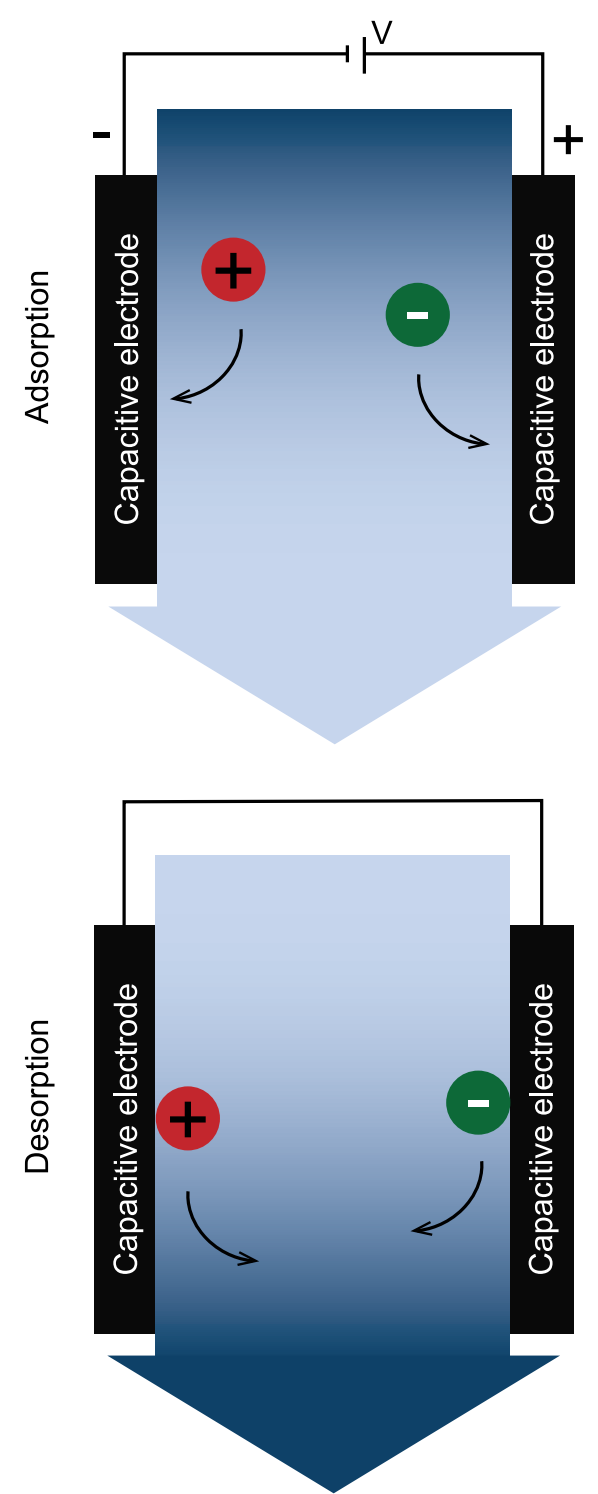

Inverted

capacitive deionization

(iCDI)
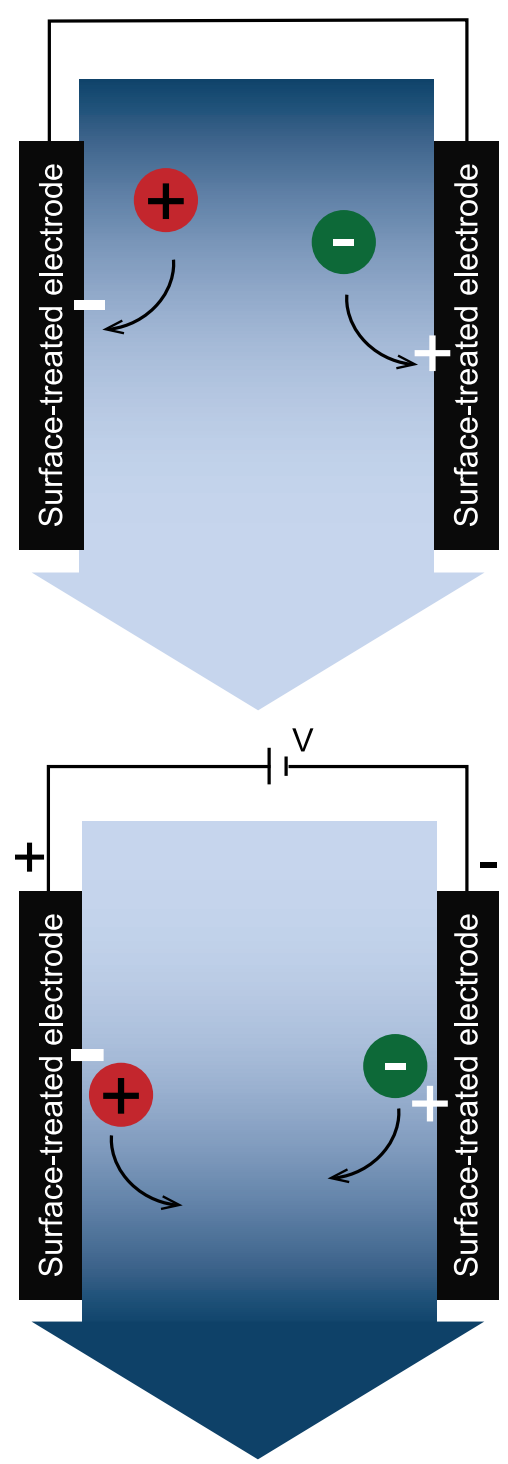

Figure 1.2. Schematic of capacitive deionization (left) and inverted capacitive deionization (right).

Where CDI is efficient and effective for the separation of ions (the electric power scales with the amount of separated ions), it is less efficient at higher ionic strengths, which would typically be present during food (ingredient) processing. In addition, the selective removal of specific molecules from complex mixtures is still poor. As an alternative, inverted CDI (iCDI) has been developed (Figure 1.2, right panels), in which ions adsorb passively onto a surface in the absence of electric current. Electric power is only needed to desorb the ions, which can be relatively efficient since the release only requires ionic transport over a small distance (in the order of the double 
layer thickness) (Gao et al., 2015a). At the same time, it may allow for specific interactions between the target molecules and the adsorptive surface if modified accordingly, resulting in the selective removal of specific components.

Although electrochemical separation processes for ionic separations have gained significant attention, and the understanding of the underlying principles has increased drastically in the past decade, insight about separation of larger molecules such as proteins is still very scarce. Therefore, in this thesis we focus on selective isolation of proteins using electrochemical principles. This includes the identification and quantification of the underlying phenomena, and definition of guidelines to design processes based on these principles, also for practical applications.

The use of electric stimuli for the separation of ions and proteins is reviewed in Chapter 2. In addition, measurement techniques are discussed that can be used to analyze the effects of the electric stimuli on the adsorption and desorption of macromolecules. Some of these techniques are used in later chapters.

In Chapter 3 we present an exergy analysis of membrane CDI using activated carbon electrodes. This concept is further extended to electrodes containing charged polymers in Chapter 4. Both the exergy analysis as well as the incorporation of polystyrene sulfonate and poly(diallyl dimethyl ammonium chloride) using a styrene butadiene rubber as binder are new approaches for CDI. In addition to that, we introduce a novel processing type, which will be referred to as inverted membrane CDI. In Chapter 5 we describe the desalination of protein containing streams using hierarchical carbon materials and highlight surface modification with zwitterionic polymer brushes to reduce protein fouling and thus improve electrode lifetime.

In Chapter 6 we take this one step further, and show how protein and salt adsorption to cationic and anionic polyelectrolytes can be reversed using an electric potential. The impact of the electrode potential on protein ad- and desorption was further studied using surface plasmon resonance in Chapter 7 and related changes in electrostatic and hydration repulsion, induced by the electrode potential, were analyzed using atomic force spectroscopy and electrowetting. Protein adsorption to carbon materials can be measured by reflectometry, and for that we prepared graphene oxide coated silicon substrates in Chapter 8.

The general discussion in Chapter 9 summarizes the main findings in this thesis and puts them in the wider context of separation processes, and gives a perspective towards future developments needed to bring the technology to fruition. 



\section{CHAPTER 2}

ELECTROCHEMICALLY DRIVEN

ADSORPTIVE SEPARATION TECHNIQUES:

FROM IONS TO PROTEINS AND CELLS

IN LIQUID STREAMS

Separation processes are of utmost importance for most industrial processes in the chemical, food, and pharma sectors. Electrochemical separation technologies gain more and more attention especially for the desalination of water, as they potentially lead to a reduction in the environmental footprint of the process. For salt, many different electrochemical desalination architectures and electrode designs have been proposed. Also other target molecules have been investigated, such as carboxylates, amino acids, proteins and whole cells. We note that the efforts are still scattered; therefore in this review we bundle and summarize the technological state of the art, and the application possibilities for capacitive as well as faradaic separation technologies for various targets. From this it is clear that there are still a lot of options that have been underused so far, and that electrochemical separation processes could play a key role in the separation processes needed for a sustainable future.

This chapter has been submitted as:

P. A. Fritz, R. M. Boom, and K. Schroën, Electrochemically driven adsorptive separation techniques: from ions to proteins and cells in liquid streams. 


\subsection{Introduction}

Numerous separation methods such as precipitation/crystallization, extraction, adsorption, and membrane filtration have been developed for purification of valuable products from liquid streams, or removal of impurities (Mandal and Kulkarni, 2011). Based on theoretical evaluations, adsorptive separation is most efficient for the removal or isolation of minor components (Giddings, 1991) and is commonly applied to remove pollutants (e.g. heavy metals) from water (Ali, 2014) or to isolate products of high value (e.g. proteins and peptides) (Geng and Wang, 2008; Hage et al., 2012). It is also applied on very large scales, as for example in the separation of glucosefructose (Cheng and Lee, 1992), olefins and paraffins (Herden et al., 1984) or isomeric mixtures (Guo and Zheng, 2009).

During adsorption a target molecule in solution first diffuses across a hydrodynamic boundary layer surrounding the stationary solid support, possibly followed by diffusion into the support, and finally by ad- and desorption to and from this interface (Qiu et al., 2009). Key factors for ad- and desorption are the polarity and charge of the surface and target molecule (Van der Waals, electrostatic, hydration forces), whereas also complexation, ion exchange and microprecipitation can play a role (Hokkanen and Bhatnagar, 2016). Thus, different molecules in the liquid (mobile) phase have different affinities for the stationary phase and can either be physi-, chemior electrosorbed.

In most cases, adsorption is an exothermic process and desorption is endothermic. For ions, typical adsorption energies to an ion exchange resin range between 0.6 and $25 \mathrm{~kJ}^{-1} \mathrm{~mol}^{-1}$, and the heat of adsorption can be as low as -24 kJ.mol-1 (Inglezakis and Zorpas, 2012; Sahai, 2000) depending on the charge of the ion and the substrate. If the adsorbent is more complex as in the case of proteins, multiple contact points in combination with structural rearrangements of the molecule leads to heats of adsorption as low as $-1200 \mathrm{~kJ} \cdot \mathrm{mol}^{-1}$ at initial adsorption (Arai and Norde, 1990). Other sources report binding free energies between -20 and $-50 \mathrm{~kJ} \cdot \mathrm{mol}^{-1}$ for individual amino acids (Kokh et al., 2010), and interaction energies of

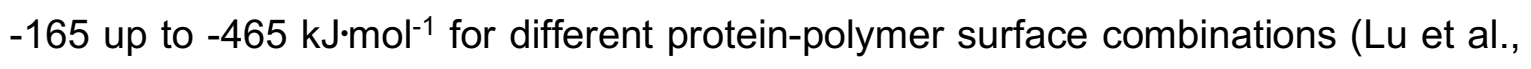
1992). 
Surface interactions can be influenced by the temperature, $\mathrm{pH}$, and ionic strength of the solution and for the product release by desorption, these environmental conditions need to be changed. In chromatography, this requires the use of extreme $\mathrm{pH}$ or ionic strengths (e.g. acidic or basic solutions or solvents) that may have a negative effect on sustainability of the process, and on the quality of the product. Recent innovations in "green chromatography" propose the use of stimuli responsive resins, that change their physical properties upon the application of other external stimuli such as temperature, $\mathrm{pH}$, magnetic- or electric fields to improve separation processes (Terefe et al., 2014). Although this influences the ad- desorption part of the process, it does not influence the diffusion part, and that can be rather time consuming. In order to accelerate this, an additional driving force would be needed such as an electric field.

In this review, we focus on the use of an electric field as stimulus in adsorptive separation processes, in which either capacitive or faradaic currents are used to store charged molecules in the double layer, or remove them, or to alter the stationary phase properties. Different technologies have been developed that make use of this concept: capacitive deionization, electrochemically modulated liquid chromatography (potentiostatic chromatography, elution voltammetry) (Blaedel and Strohl, 1965; Collins and Arrigan, 2009; Eads and Payne, 1964; Ge et al., 1991; Yamini et al., 2014), electrochemical solid phase (micro)extraction (Breadmore, 2007; Collins and Arrigan, 2009; Liljegren et al., 2002; Yamini et al., 2014), and electrokinetic trapping (Yamini et al., 2014) but the available options that could be envisioned are far from exhausted with this list. All these processes employ a surface to adsorb or immobilize the target molecules, and still a lot of progress can be made in terms of modulating the interactions occurring close to the surface, either through surface modification, or by influencing the interactions that take place at close range.

To be clear, we exclude permeation based electrochemical membrane techniques such as electro-dialysis, electro-osmosis, electro-microfiltration and electrokinetic membrane extraction, and refer interested readers through to the following reviews (Breadmore, 2007; Collins and Arrigan, 2009; Kubáň et al., 2010; Morales-Cid et al., 2010; Nikitas, 2000; Pedersen-bjergaard and Rasmussen, 2008; Petersen et al., 2011; Yamini et al., 2014). 


\subsection{Surface interactions acting during electro-adsorptive processes}

Interactions at the solid-liquid interface are key for adsorptive separation processes and the nature and strength of the interactions depend on multiple factors. For ions the dominant factor are electrostatic interactions, but for larger molecules, the DLVO theory suggests that Van der Waals and electrostatic interactions are essential, and further hydration forces play a role (Parsegian and Zemb, 2011; Parsons et al., 2011). Especially the latter two are strongly influenced by an electric potential applied to the interface, and are discussed in the next section. Van der Waals forces, as shown in Figure $2.2 \mathrm{e}$, will not be covered since they are related to the dipole of the molecules in the surface and not influenced by the surface potential at low ionic strength (Israelachvili, 2011), and thus cannot be used in a cyclic electrically driven adsorption/desorption process.

\subsubsection{The electric double layer and electrostatic interaction energies}

The charge as function of the distance to a charged solid wall is generally described with a double layer model. A widely shared view is that the double layer consists of a concentrated layer of specifically adsorbed ions near the wall called the Stern layer, which becomes more diffuse when moving from the wall, and ultimately resembles the bulk electrolyte concentration far from the wall (GCS-model; Figure 2.1 a).

For solid interfaces that are electrically charged, the current involved in building the double layer is referred to as capacitive current: no electron is exchanged over the interface; charge is only accumulated (Biesheuvel and Dykstra, 2018). The potential drop at an electrode interface can be modelled using the Debye screening length, $\kappa^{-1}$ (Equation 2.1) and the Poisson-Boltzmann equation (Equation 2.2).

$$
\begin{gathered}
\kappa=\sqrt{\frac{2 n_{0} e^{2}}{\epsilon \epsilon_{0} k T}} \\
\frac{d^{2} \psi}{d z^{2}}=\kappa^{2} \frac{k T}{e} \sinh \left(\frac{e}{k T} \psi\right)
\end{gathered}
$$



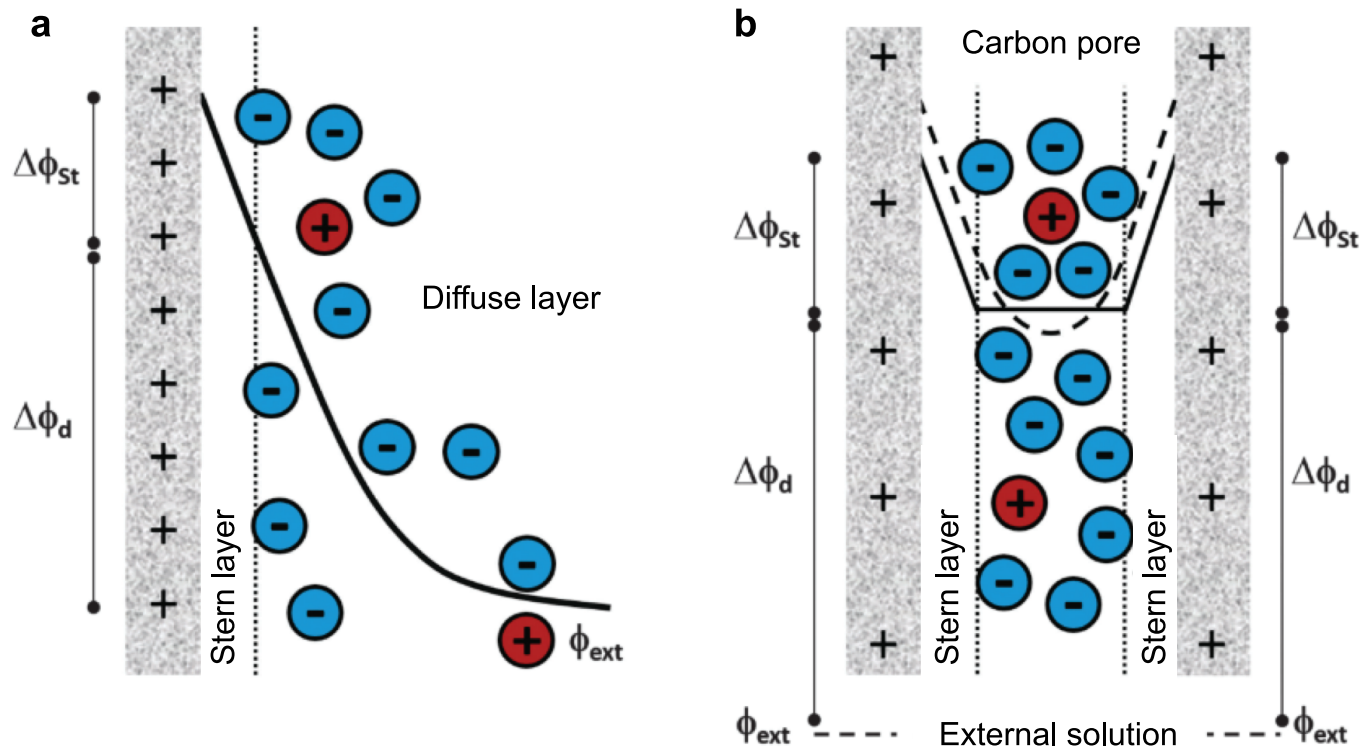

Figure 2.1. Ion storage in a non-overlapping (Gouy-Chapman-Stern model) and $\mathbf{b}$ overlapping double layers (modified Donnan model). Reproduced with permission from Suss et al., 2015.

With a larger surface charge, the potential drop increases (Figure 2.2 a), and expands further. The electrostatic interaction energies between two charged plates at a finite distance from each other can be calculated using Equation 2.3.

$$
V_{E}=-\int_{\infty}^{D}\left\{2 n_{0} k T\left[\cosh \left(\frac{e \psi}{k T}\right)-1\right]-\frac{\epsilon \epsilon_{0}}{2}\left(\frac{d \psi}{d z}\right)^{2}\right\} d z
$$

Like charges repel each other, while unlike charges attract each other upon approach, indicating the possibility for adsorption and desorption between the two plates. In Figure $2.2 \mathrm{c}$ and $\mathrm{d}$, the electrostatic repulsion between two negatively charged flat plates is shown as a function of the distance $D$ between the two plates, for constant potential and constant charge boundary conditions, respectively.

Experimental studies to measure the double layer potential with open circuit and with applied potential have been done with atomic force spectroscopy (Butt, 2007; Fermín, 2006; Papastavrou, 2010). At the potential of zero force, the size of the double layer is minimal but extends if the applied potential is shifted to more negative or more positive values, indicating an increase in ion storage capacity. 


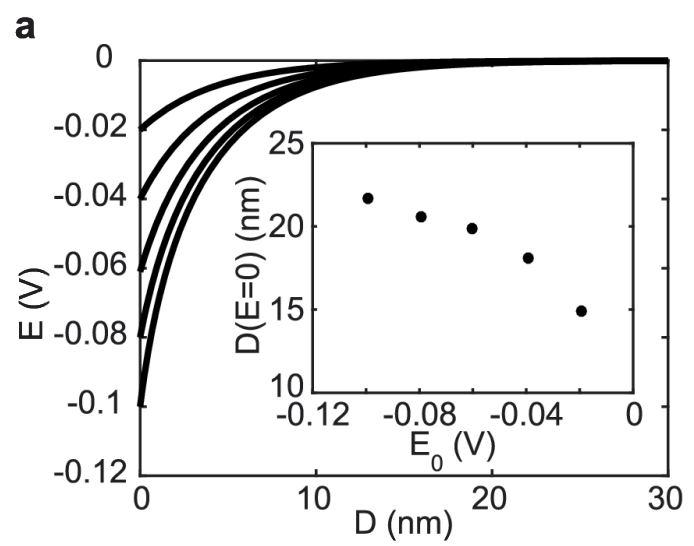

C

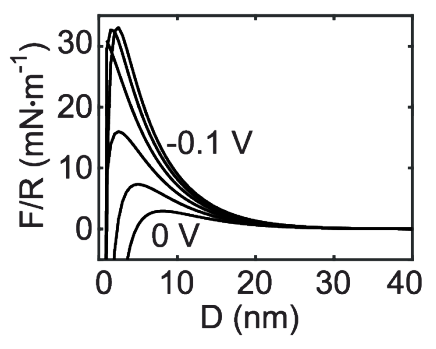

d

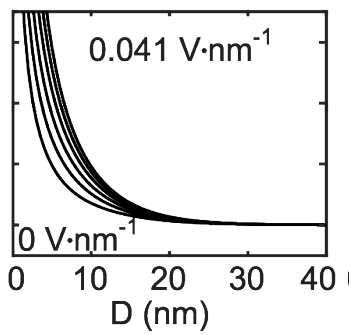

b

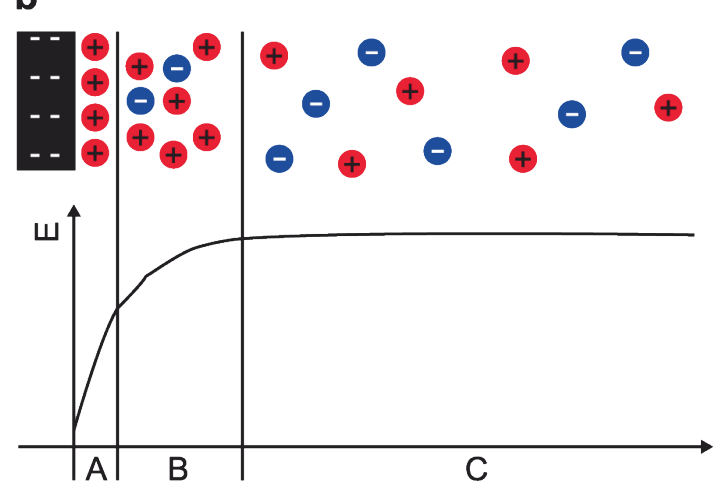

e

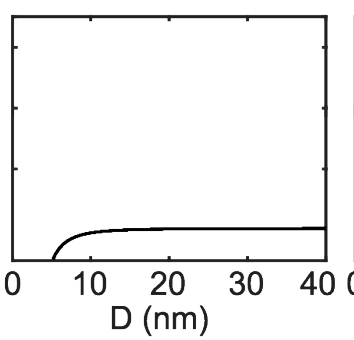

f

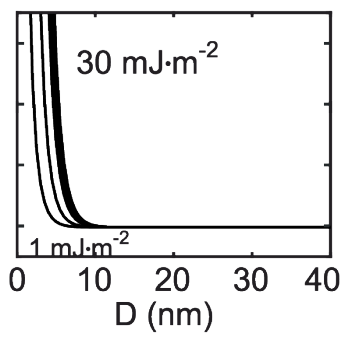

Figure 2.2 a Double layer potential $(E)$ profile from the electrode interface ( $E_{0}$ between -0.1 and $-0.02 \mathrm{~V}$ ) to the electrolyte solution. Inset: Distance at which the potential drops to $0 \mathrm{~V}$ (cutoff: $-5 \cdot 10^{-4} \mathrm{~V}$ ). b Schematic of ion distribution at electrode interface within the electric double layer and potential profile at interface. c Electrostatic interaction energy determined for constant potential boundary conditions, gold electrode was held at $0,-0.2,-0.4,-0.6,-0.8$ or $-1.0 \mathrm{~V}$ and silica probe set at $-0.06 \mathrm{~V}$. d Constant charge boundary conditions were determined using Graham's equation for values between 0 and $0.041 \mathrm{~V} \cdot \mathrm{nm}^{-1}$ for the gold electrode, and $-0.0173 \mathrm{~V} \cdot \mathrm{nm}^{-1}$ for the silica surface. $\mathbf{e}$ Van der Waals attraction for a Hamaker constant of $5.7 \cdot 10^{-20} \mathrm{~J}$, and $\mathbf{f}$ hydration repulsion for hydration repulsion amplitude between 1 and $30 \mathrm{~mJ} \cdot \mathrm{m}-2$ with decay length of $1.29 \mathrm{~nm}$.

Please note that the double layer for semiconductors (e.g. ITO) (Bos et al., 1994; Hu et al., 1997) is smaller compared to that of conductive surfaces such as gold (Barten et al., 2003a; Duval et al., 2001; Fréchette and Vanderlick, 2001; Giesbers et al., 2002; Hillier et al., 1996; Kuznetsov and Papastavrou, 2012; Moulton et al., 2003; RoquesCarmes et al., 2006; Zhou and Xie, 1999; Zhou et al., 2000), stainless steel (Beaglehole et al., 1998), or pyrolytic carbon (Holmstrom et al., 1998), since most of the potential drop occurs in the semiconductor layer itself (Bos et al., 1994).

In nanoporous materials such as activated carbon $(<2 \mathrm{~nm})$, the double layer is not fully developed, since the diameter of the pores are smaller than the distance over which the potential drops, and therefore the double layers strongly overlap. In this case the structure can be described using the modified Donnan model (Figure 2.1 b). Further descriptions and summaries about the modified Donnan model can found in the following references (Biesheuvel et al., 2011, 2014; Suss et al., 2015). 


\subsubsection{Hydration forces}

As mentioned, hydration forces are important in ad- as well as desorption events, especially for macromolecules such as proteins in an aqueous environment (Israelachvili and Pashley, 1983). Hydration repulsion can be attributed to primary, secondary, and structural hydration forces (Figure $2.2 \mathrm{f}$ ), related to the water molecules that are directly bound to the interface and/or protein molecules, water molecules in ionic hydration shells, and steric effects due to the finite volume of the solvent molecules (Kilpatrick et al., 2013; Parsegian and Zemb, 2011). If the orientation and density of water molecules at the interface is different compared to the bulk distributions, the force to displace a water molecule by an adsorbing target molecule is impacted (Besseling, 1997). At the surface of an electrode, the orientation and density of water molecules can be influenced by the applied potential (Velasco-Velez et al., 2014), and thus ad- and desorption of e.g. proteins can be influenced (Chapter 7).

In bulk water, the hydrogen-bonding-to-dispersion-forces have a ratio of 70:30, which relates to a contact angle of $63^{\circ}$. A surface exhibiting a lower contact angle is denoted hydrophilic; a surface with a higher contact angle is hydrophobic (Vogler, 2012). This implies that hydration interactions can be measured using electrowetting (Mugele and Baret, 2005), in which the contact angle is a function of the applied electric potential (Vogler, 2012). Usually, the contact angle and the Gibb's free energy decrease when a potential is applied, which implies that the work of water adhesion increases, indicative of more hydration repulsion.

\subsection{Capacitive separation processes}

\subsubsection{Capacitive separation of ions (Capacitive deionization)}

For ion separation, porous carbon materials are used in capacitive deionization (CDI) systems. The most commonly used system contains two carbon electrodes opposite from each other, separated by a non-conductive spacer. The feed flows between the two electrodes through the spacer (Figure $2.3 \mathrm{a}$ ); while applying constant potential or current, charged ions dissolved in the feed are stored in the double layer at the electrode interfaces and thus the feed flow is desalinated. For regeneration of the electrodes the current or potential is reversed or switched off, and the ions are 
released from the interface generating a brine (Huang et al., 2013; Porada et al., 2013; Suss et al., 2015). Depending on the carbon material, electrosorption capacity values range from 0.25 for simple activated carbon to $24.2 \mathrm{mg} \cdot \mathrm{g}^{-1}$ in graphene aerogels in which nanopores play a crucial role (Jia and Zhang, 2016; Oladunni et al., 2018).

As an extension, CDI in flow-through mode, (Figure $2.3 \mathrm{e}$ ) or with carbon flow electrodes (Figure $2.3 \mathrm{~h}-\mathbf{j}$ ) have been developed, and besides carbon intercalation materials are currently investigated to boost the desalination capability (Figure $2.3 \mathbf{f}$ and $\mathbf{g}$ ) (Suss et al., 2012). In these materials, ions are captured through redox reactions at the interface. Although Biesheuvel et al. (Biesheuvel and Dykstra, 2018) argue that this can be considered capacitive ion storage (the electro composition stays the same, and no electrons are transferred), we follow other reviews and discuss intercalation materials in the context of faradaic separation processes (Zhang et al., 2018a).

By inserting ion selective membranes (Figure 2.3 b) between the electrodes, (Biesheuvel and van der Wal, 2010) or coating the electrodes (Yang et al., 2013), the charge efficiency of flow between desalination processes can be improved, and the removal of specific ions such as nitrate promoted (Kim and Choi, 2012; Kim et al., 2013; Yeo and Choi, 2013). Furthermore, it allows the successful desalination of more complex feeds such as brackish water containing proteins (Zhang et al., 2018b) or micro-organisms (El-Deen et al., 2016a), or even biomass hydrolysates containing sugars, organic acids or furans (Huyskens et al., 2013). Besides, $\mathrm{ZnCl}_{2}$ was separated from insulin samples (Jung et al., 2012) while acetic and sulfuric acid was removed from hydrolysate (Kim et al., 2012). Furthermore, it is good to mention that CDI technology on larger scale has been reported (Lee et al., 2006).

In contrast to conventional CDI in which adsorption takes place due to an applied potential or current, in inverted CDI, modified electrodes with additional surface charges (anode: net negative; cathode: net positive) are used to store ions (Figure 2.3 c). This means that adsorption occurs at 0 V (Gao et al., 2015a, 2015b) or the potential of zero charge (Gao et al., 2018), whereas desorption is activated by depolarizing the electrodes electrochemically. This saves energy during loading especially at higher solution conductivity, and allows for the selective adsorption of specific ions, depending on the groups present on the electrodes. 
a

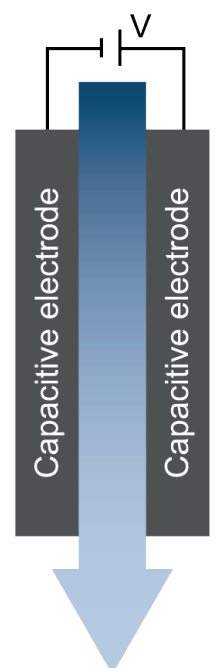

CDI (Flow between electrodes) b

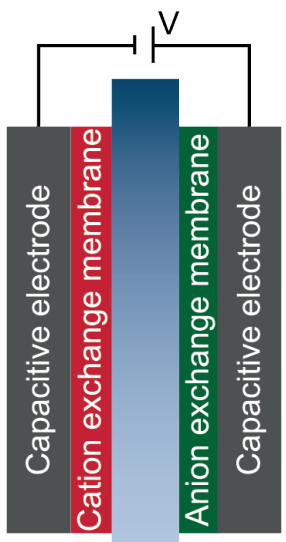

Membrane CDI

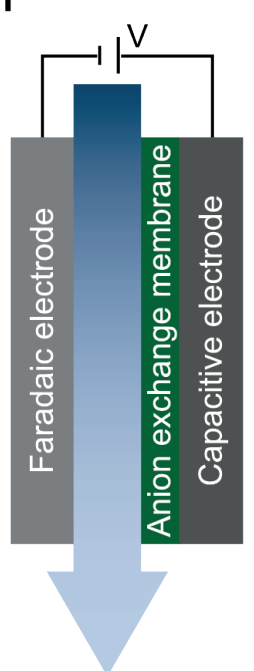

Hybrid CDI g

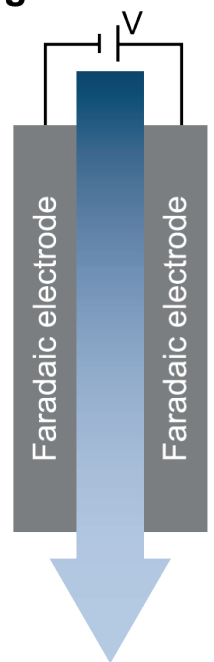

Desalination
battery
C

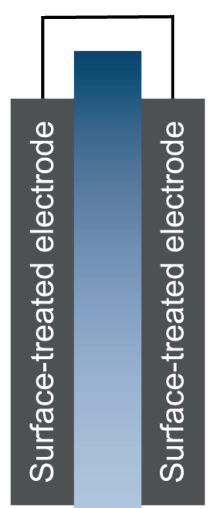

Inverted CDI d
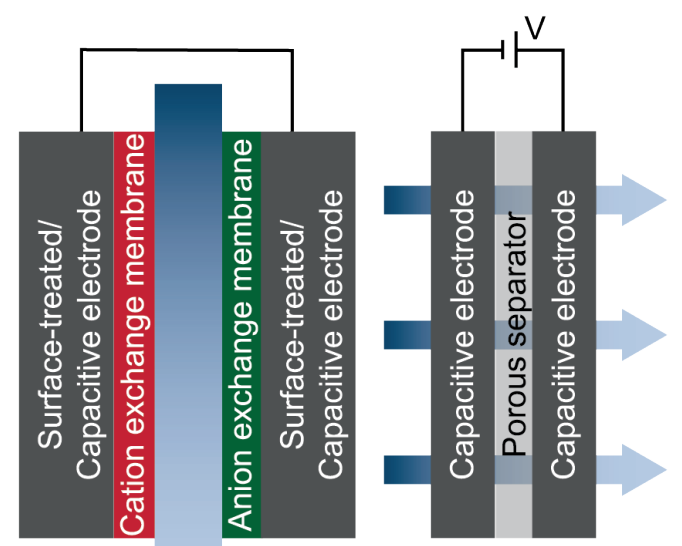

Flow-through electrode h

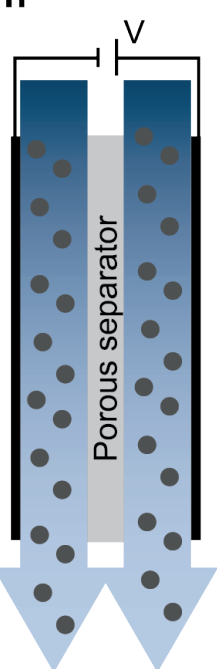

CDI with flow electrodes (Feed in electrodes) i

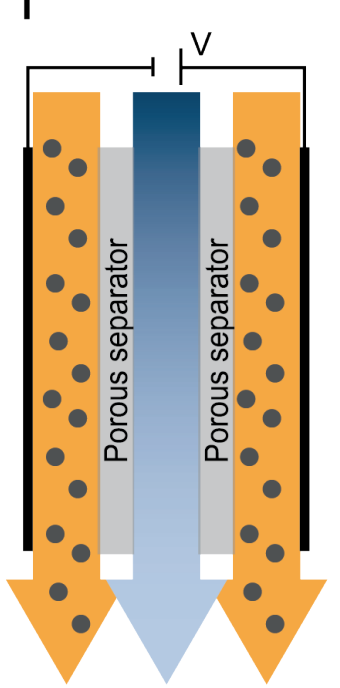

CDI with flow electrodes (Feed between electrodes)

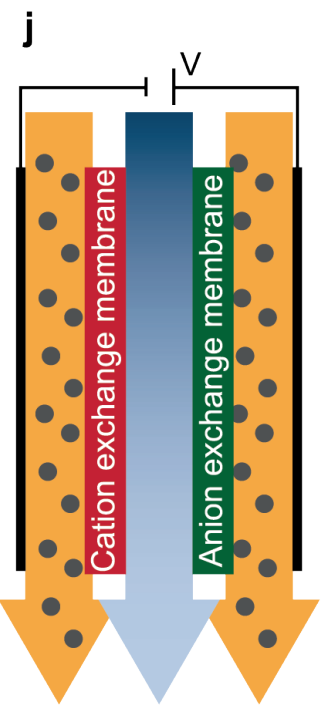

MCDI with flow electrodes

Figure 2.3. Capacitive deionization technologies ranging from flow-between $(\mathbf{a}-\mathbf{d}, \mathbf{f}, \mathbf{g})$ to flow-through (e) and flow electrode $(\mathbf{h}-\mathbf{j})$ set ups, using either capacitive or faradic ion storage principles. Modified from Suss et al., 2015.

For iCDI, in literature carbon materials modified with tetraethyl orthosilicate and/or nitric acid (Gao et al., 2015a, 2015b), or polystyrene sulfonate (Fritz et al., 2019a) have been used to increase the net negative charge, and the opposite charge was increased with ethylenediamine (Gao et al., 2015a) or poly(diallyldimethylammoniumchloride) (Chapter 6). Activated carbon electrodes were treated with cetrimonium bromide and sodium dodecyl benzene sulfonate for the selective removal 
of nitrate (Oyarzun et al., 2018; Palko et al., 2018). A theoretical overview of iCDI desalination systems and the influence of chemical surface charges is available from Biesheuvel et al. (Biesheuvel et al., 2015).

The efficiency of an inverted CDI process can be further increased by inserting ion exchange membranes, possibly in combination with unmodified and modified activated carbon electrodes (Figure $2.3 \mathrm{~d}$ ). The separation performance of this inverted membrane CDI set up was competitive with conventional CDI using the same electrodes (Fritz et al., 2018, 2019a).

\subsubsection{Capacitive separation of larger molecules}

Capacitive current can also be used to alternate adsorption of colloids and macromolecules (Figure 2.4) as investigated using reflectometry, ellipsometry, or surface plasmon resonance equipped with an electro chemical cell (as compiled in Table 2.1). For example the adsorption of polyvinyl pyridine (Figure 2.4 a) (Barten et al., 2003b; Kleijn et al., 2004), poly(propylene imine) dendrimer (Figure 2.4 b) (Kleijn et al., 2004), and of poly(vinyl imidazole) (Roques-Carmes et al., 2006) (positive at low $\mathrm{pH}$ ) increased with the cathodic potential. Thiols could be chemi- or physisorbed onto mercury depending on the applied potential (Muskal and Mandler, 2000), and AFM studies revealed attractive and repulsive forces at positive and negative potentials, respectively, acting on negatively charged silica particles (Kuznetsov and Papastavrou, 2012; Rentsch et al., 2007), and DNA (Erdmann et al., 2009).

For the adsorption of blood proteins (Beaglehole et al., 1998; Holmstrom et al., 1998; Mattson and Smith, 1973; Morrissey et al., 1976; Moulton et al., 2003; Srinivasan and Stoner, 1970), lysozyme (Figure 2.4 c and d) (Kleijn et al., 2004; Xie et al., 2013) and horseradish peroxidase (Cole et al., 2007) a similar trend was found: the adsorption increased with increasing potential relative to the protein charge. Desorption was only detected for DNA (Hook et al., 2006; Jiang et al., 2004; Wang et al., 1999) and $\beta$-lactoglobulin (Chapter 7). Difficulties for desorption in other cases were mostly related to multiple contact points, effectively rendering the adsorption irreversible. Although mostly not considered, for an electrochemical protein separation process, the change in surface tension induced by the applied potential due to hydrostatic and electrostatic forces was found to be of great importance (Chapter 7 ). 
a

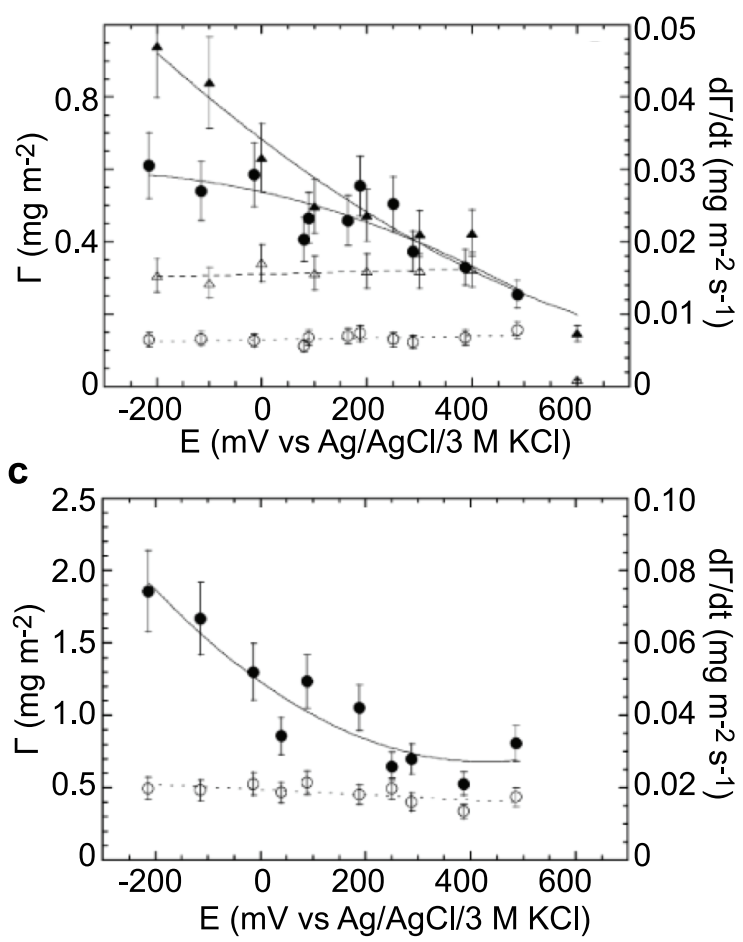

b

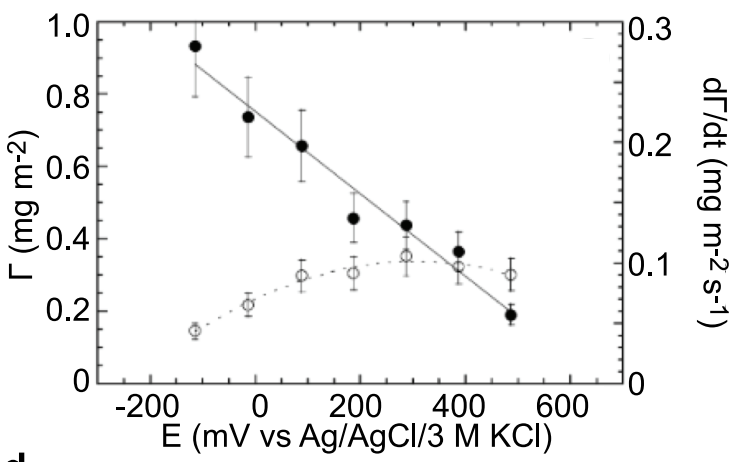

d

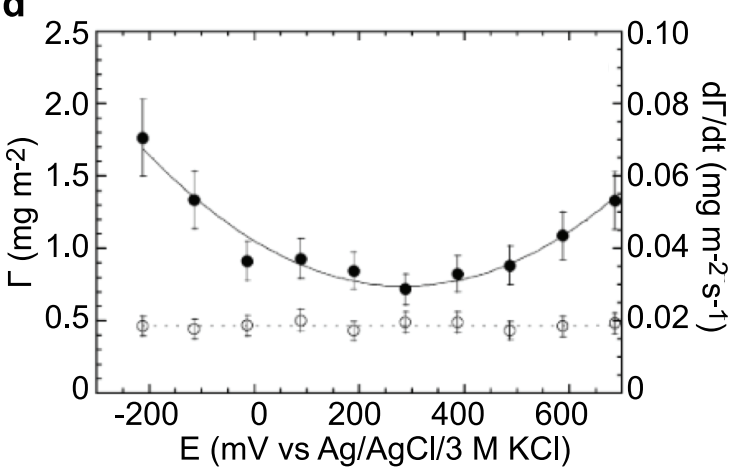

Figure 2.4. Adsorbed amounts (closed symbols) and adsorption rates (open symbols) of charged macromolecules to gold electrodes as function of applied potential. a PVP+, 12k (b, O), concentration 1 $\mathrm{mg}^{\circ} \mathrm{L}^{-1}$, and $124 \mathrm{k}(2,4)$, concentration $5 \mathrm{mg}^{\circ} \mathrm{L}^{-1}, \mathrm{pH}$ 6.4. b Adsorption of DAB-64 from a $5 \mathrm{mg}^{\circ} \mathrm{L}^{-1}$ solution at $\mathrm{pH}$ 6.9. (c and d) Adsorption of lysozyme from a $3 \mathrm{mg}^{\circ} \mathrm{L}^{-1}$ solution at $\mathrm{pH} 6.4$ and $\mathrm{pH} 5$, respectively. Background electrolyte $1 \mathrm{mM} \mathrm{KNO}_{3}$. The lines are to guide the eye. (Reproduced with permission from Kleijn et al., 2004).

A first capacitive protein separation process was proposed by Fritz et al. (Figure 2.5), ad- and desorption of whey protein isolate to and from various electrodes that may carry a polyelectrolyte layer could be controlled by an externally applied electric potential. Upon applying a potential, the UV signal that is linked to protein concentration drops, while conductivity that corresponds to salt increases (Figure 2.5 a and b). Since protein ad- and desorption occurred at opposite potentials compared to salt ad- and desorption, this makes this process also useful for desalination of proteins (Chapter 6). 
a

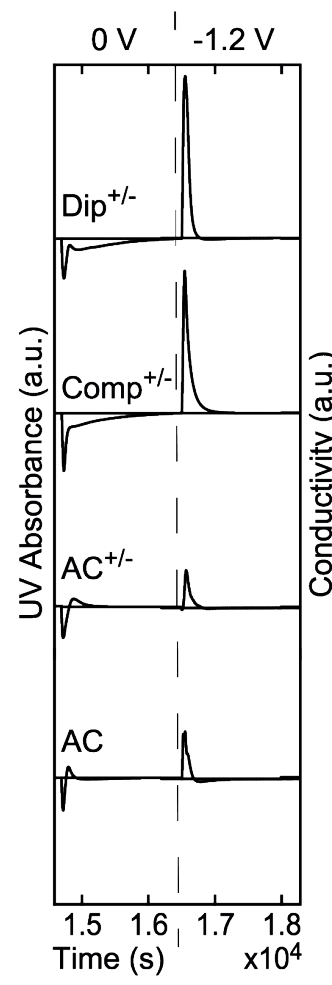

b

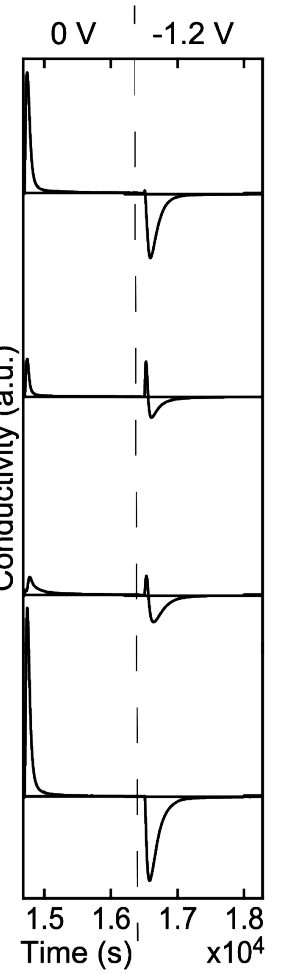

c

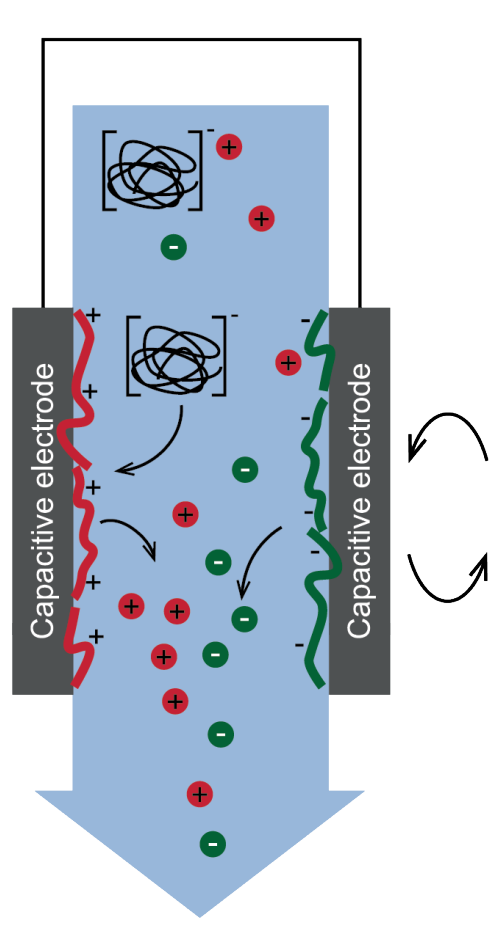

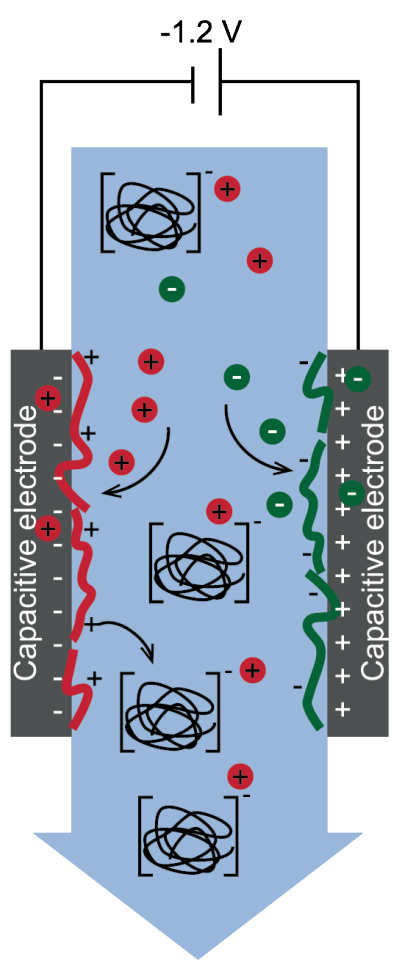

Figure 2.5. Protein and salt separation using an electrical switch. a Change in UV signal of the eluent during cycle 5. b Change in conductivity of the eluent during cycle 5. c Schematic of salt and protein adand desorption. At $0 \mathrm{~V}$ proteins adsorb and salt is released, while at a potential bias proteins desorb and salt is stored in the electrodes.

\subsubsection{Electrically responsive coatings (capacitive current)}

Capacitive current may be used to trigger conformational changes in the surface coating, and thus to indirectly promote the exchange of larger molecules (Maharjan et al., 2008; Mendes, 2008; Terefe et al., 2014). This was achieved by coating mercaptohexadecenoic acid at low surface coverage to a gold electrode (Figure 2.6). At negative potential the hexadecenoic acid chain was oriented perpendicular to the surface, whereas upon positive depolarization the carboxyl group was directed towards the electrode surface and the chain folded upon itself. Hence, the nature of the surface changed from negatively charged to hydrophobic, allowing the separation of streptavidin and avidin with isoelectric points of 5.8 and 10.5, respectively (Lahann et al., 2003; Liu et al., 2004; Ma and Shrotriya, 2012; Mu et al., 2007). The same system was also used to retain and release bacteria (Pranzetti et al., 2013); and in slightly modified form using a terminal amino group (Mu et al., 2007) as well as positively charged oligolysine peptides instead of the carboxyl groups (Yeung et al., 2010). 


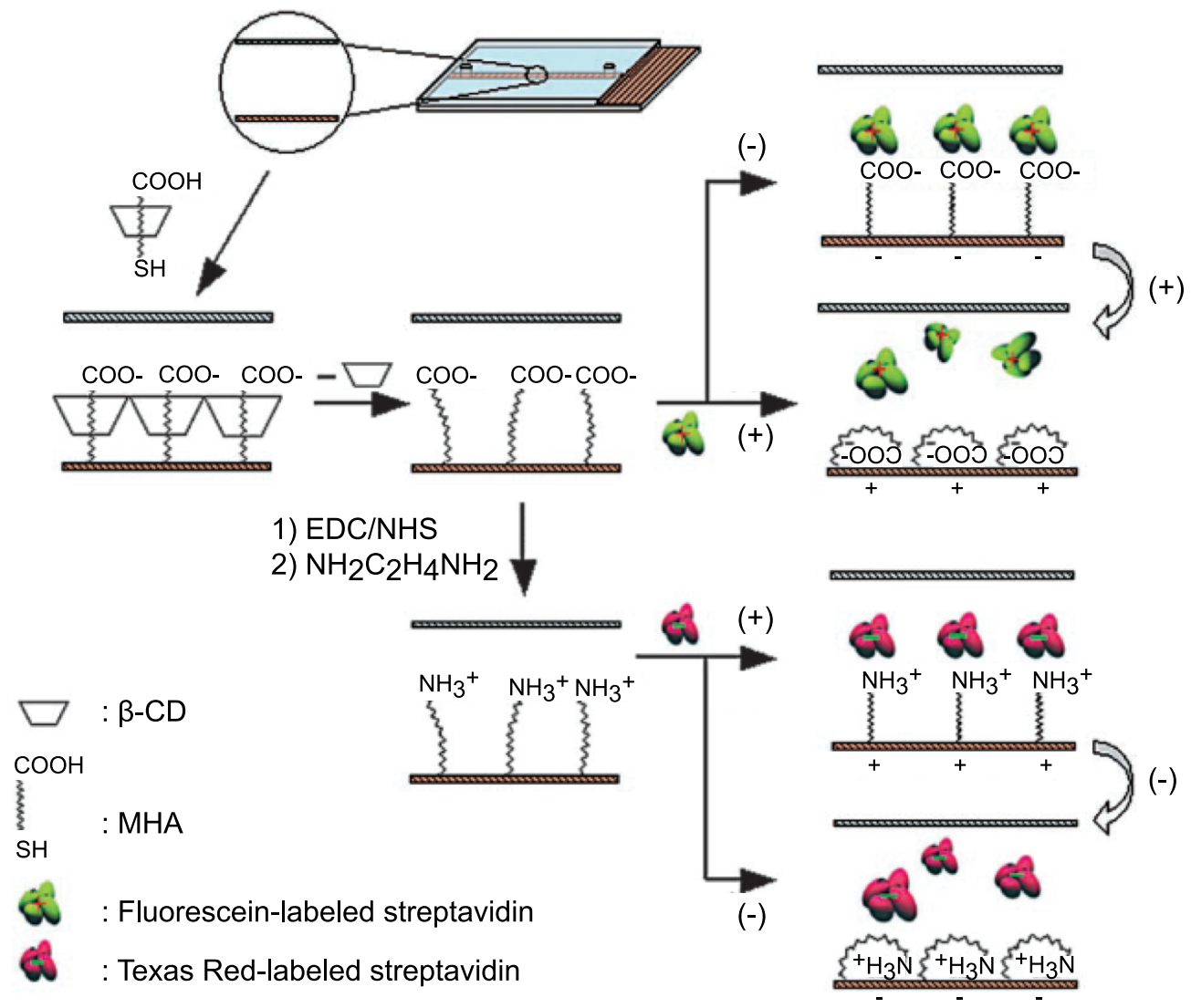

Figure 2.6. Schematic representation of electro-responsive surface coating for protein separation using a flexible hydrophobic carbon back bone and a charged head group (either $\mathrm{COO}$ or $\mathrm{NH}_{3}{ }^{+}$). Upon applying a potential, either the chain is upright, exposing the head group or bent, exposing the backbone. MHA: mercaptohexadecanoic acid (Reproduced with permission from Mu et al., 2007).

Zwitterionic phosphorylcholine was theoretically investigated; the charge ratio between the phosphate and choline group shifted as function of applied potential. This may change the interaction with Cytochrome $c$, indicating that ad- and desorption could be possible using a potential switch without impacting the structure of the protein (Xie et al., 2015). Also, multi-component, self-assembled monolayers have been tested for cell adhesion, but this adhesion is irreversible ( $\mathrm{Ng}$ et al., 2012). For electrically responsive coatings, charged polyelectrolytes (Cao et al., 2012; Fang and Szleifer, 2006; Ouyang et al., 2009) such as polystyrene sulfonate (Kawaguchi et al., 1988; Xu et al., 2012) and pyridine (Stolberg et al., 1986) have been suggested. 


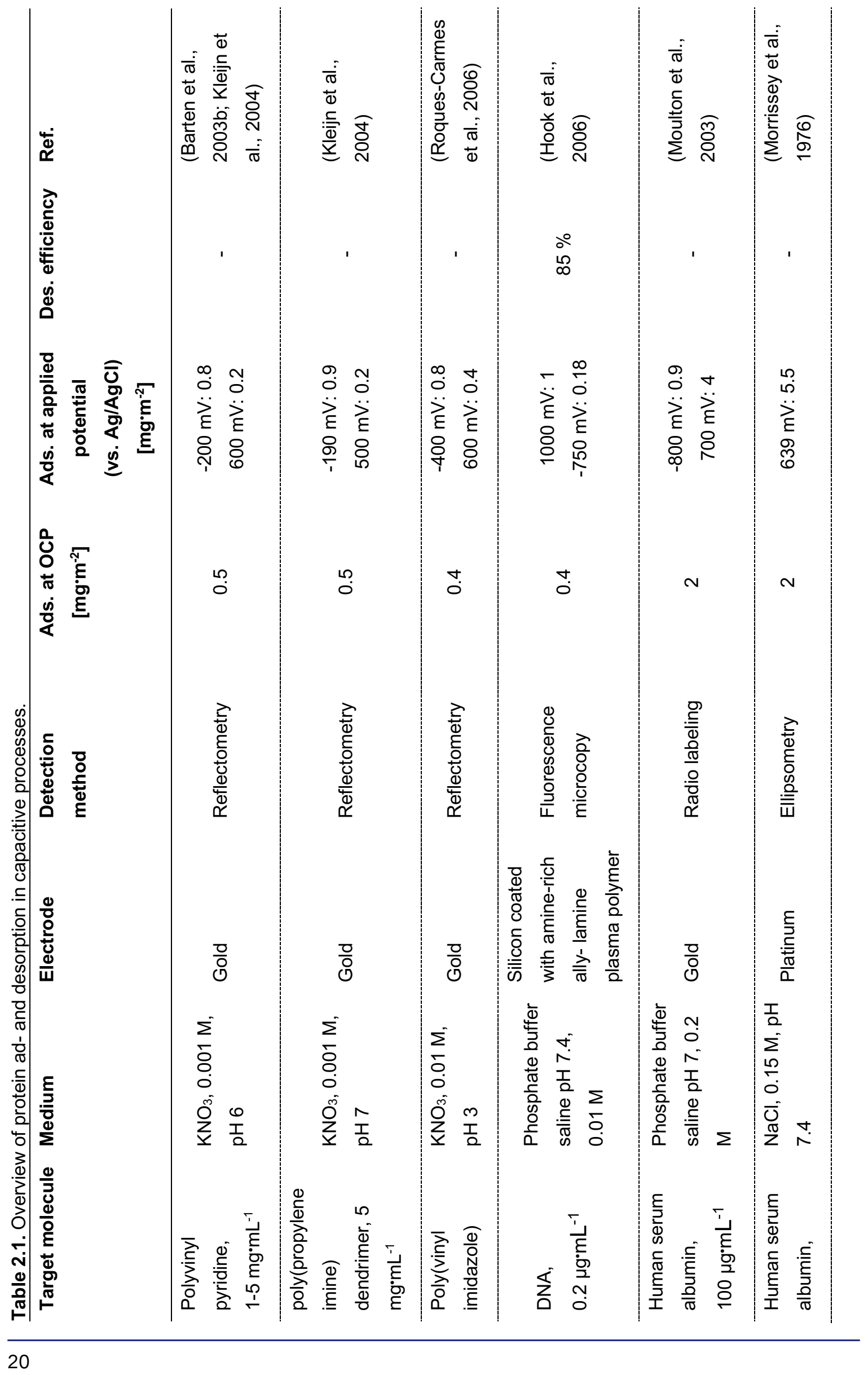




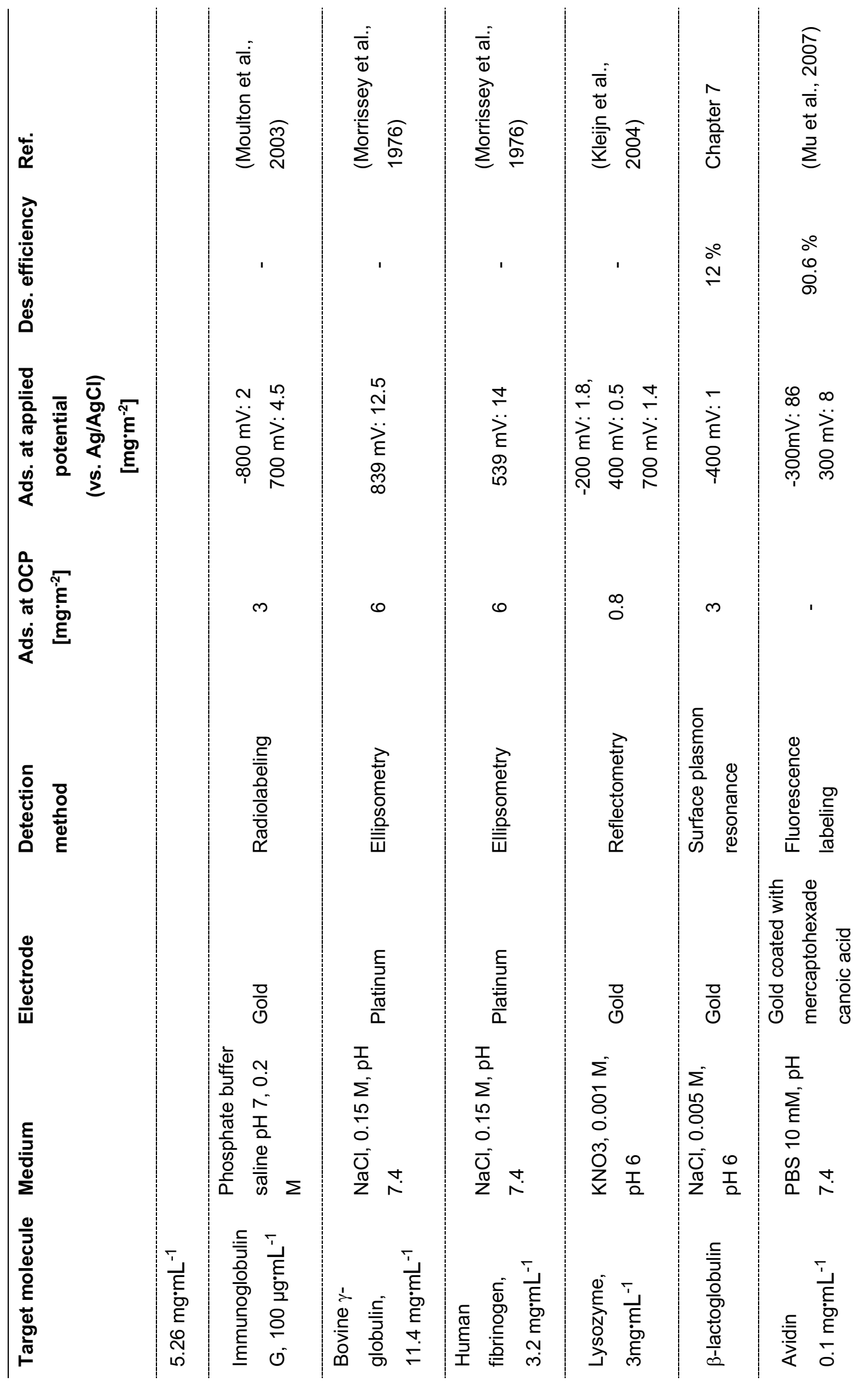




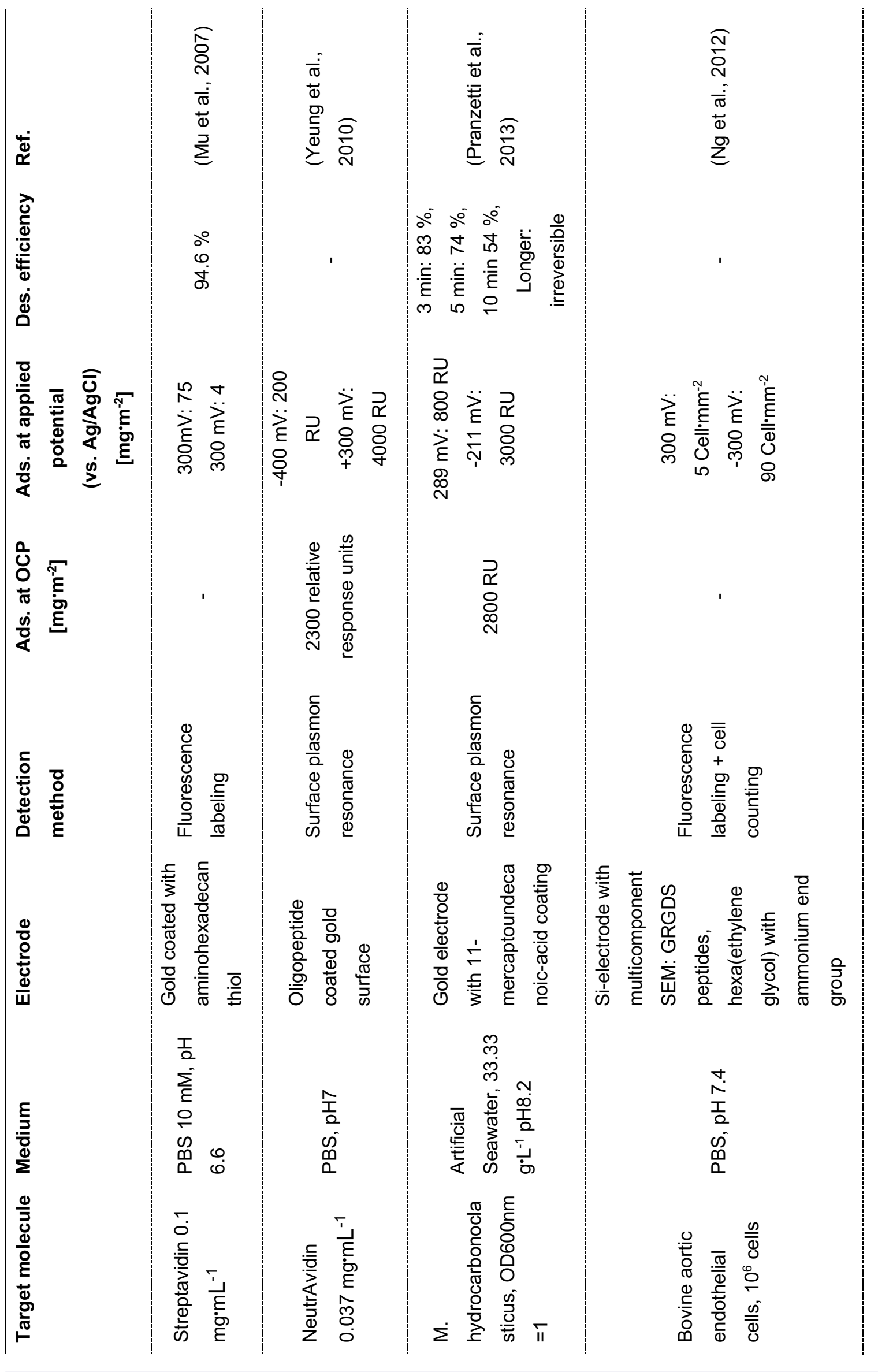




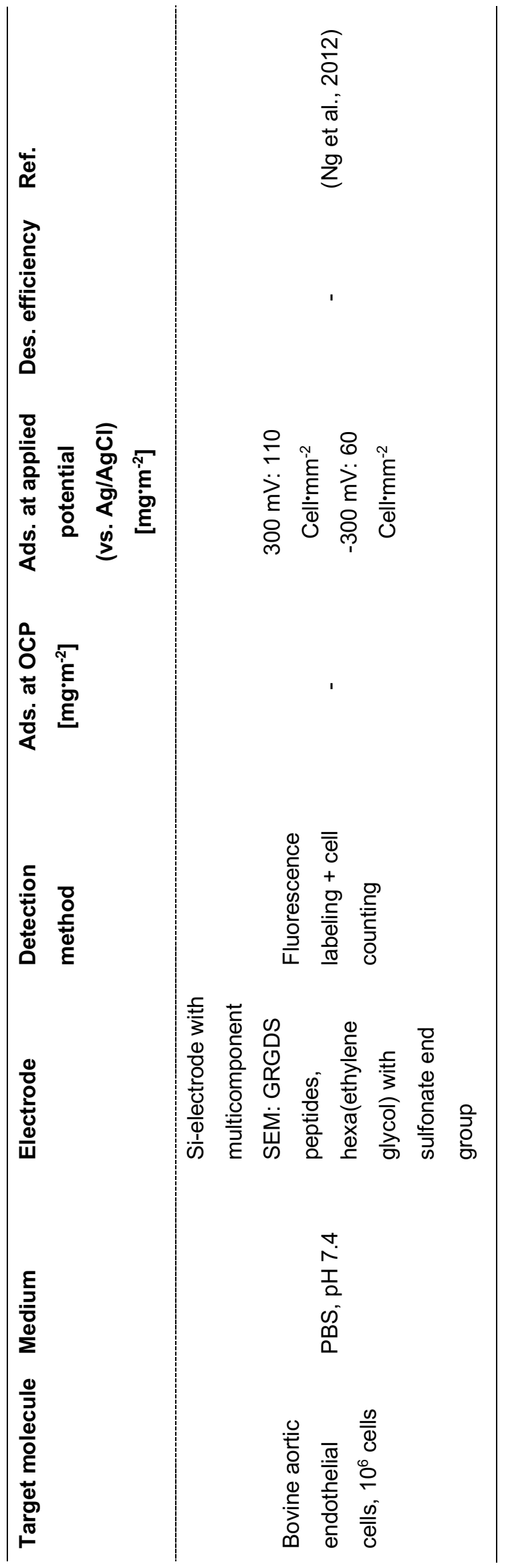




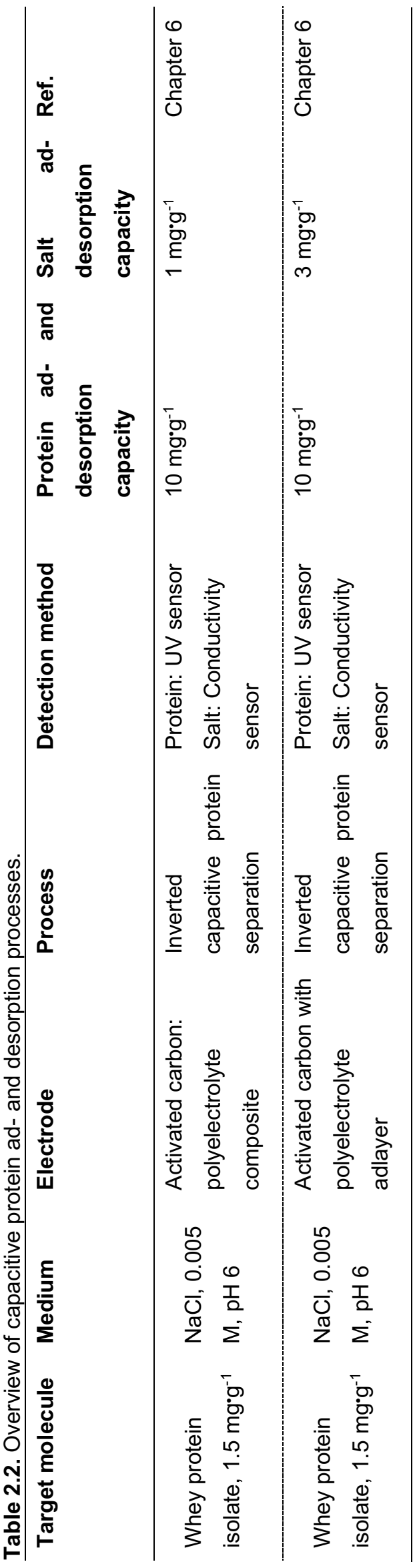




\subsection{Faradaic separation processes}

In contrast to the capacitive separation processes, the storage of charged species in faradaic separation processes is based on charge transfer. Some argue that this includes the detachment of the reaction product from the electrode after electron exchange, and thus intercalation materials or redox-active electrode coatings would be capacitive (Biesheuvel and Dykstra, 2018). However, here we maintain the classification used in previous reviews (Suss et al., 2015; Yoon et al., 2019) and limit ourselves to processes that use electrodes at which redox reactions occur leading to the storage of target molecules.

\subsubsection{Faradaic separation of ions}

Partition chromatography of metal ions by electrodeposition was reported already in the sixties (Blaedel and Strohl, 1965). The distribution coefficient $k_{D}$ between solution and stationary phase of $\mathrm{Ti}$ and $\mathrm{Pb}$ could be influenced by an applied electric potential (Nernst equation). As stationary phases, mercury (Blaedel and Strohl, 1965), amalgamated nickel, lead, noble metals, as well as other amalgamated metals, and graphite (Eads and Payne, 1964) have been reported.

Through the development of intercalation materials for batteries and supercapacitors, the storage capacity could be greatly improved. This allowed ions to be stored within the electrode material and not only at its surface. Especially for cation separation (lithium, sodium, potassium) many materials have been considered such as manganese oxide (Kanoh et al., 1991; Kim et al., 2017; Pasta et al., 2012a), ironbased phosphate (Kim et al., 2016; Pasta et al., 2012b), and hexacyanoferrate (Lee et al., 2017; Trócoli et al., 2015; Yoon et al., 2018). For chloride ions, silver and bismuth electrodes have been proposed (Hui and Yang, 2017; Nam and Choi, 2017; Pasta et al., 2012a) or two-dimensionally layered materials such as MXene (Srimuk et al., 2016) and $\mathrm{MoS}_{2}$ (Srimuk et al., 2017; Xing et al., 2017). Some intercalation electrodes can also be used for the selective removal of ions using specific conversion reactions or tunnel structure (Kim et al., 2017). For reviews on intercalation materials we refer to the appropriate manuscripts (Su and Hatton, 2017a; Suss and Presser, 2018; Yoon et al., 2019; Zhang et al., 2018a). 
Conductive and redoxactive polymers have also been considered for ionexchange voltammetry, electrochemically modulated liquid chromatography (Deinhammer et al., 1995; García et al., 2011; Liljegren and Nyholm, 2003; Liljegren et al., 2002; Wu and Pawliszyn, 2001; Wu et al., 2002), and as components in batteries (Song and Palmore, 2006), and pseudocapacitors (Arcila-Velez and Roberts, 2014). As such, polypyrrole (Figure $2.7 \mathbf{a}$ and $\mathbf{b}$ ) and polyaniline are neutral, but when partially oxidized at positive potentials they became positively charged; a negative potential returned the polymer into its neutral reduced state. This allowed the exchange of counter anions and electro-inactive analytes (Yamini et al., 2014). The ion selectivity of a polypyrrole electrode could be adapted by doping. $\mathrm{Cl}^{-}$doping led to the following preference: $\mathrm{Br}^{-}>\mathrm{SCN}^{-}>\mathrm{SO}_{4}{ }^{2-}>\mathrm{I}^{-}>\mathrm{CrO}_{4}{ }^{2-}$, whereas perchlorate doping changed the sequence to $\mathrm{SCN}^{-}>\mathrm{Br}^{-}>\mathrm{I}^{-}>\mathrm{SO}_{4}{ }^{2-}>\mathrm{CrO}_{4}{ }^{2-}$ (Ge and Wallace, 1992; Teasdale and Walfacet, 1993; Vorotyntsev et al., 1998). Cations could be adsorbed by entrapping large anions into the polypyrrole and polyaniline electrode (Figure $2.7 \mathrm{~b}$ ), since at reduced state the overall charge of the electrode was negative (Arca et al., 1995; Hasan et al., 2005). For this, anionic intercalation materials (Du et al., 2014; Wang et al., 2014a) have been discussed, and also catechol and quinones (Hepel and Dentrone, 1996).

Other suggested conductive and redox active polymers were polyampholyte (Espenscheid and Martin, 1989), sulfonate-ferrocene copolymer (Beer and Bayly, 2005; Espenscheid and Martin, 1985, 1989), poly(2,6-pyridinedicarboxylic acid) (Wang et al., 2014b) or polythiophenes (Higgins, 1997; Schaferling and Bauerle, 2004; Suarez-Herrera and Feliu, 2009; Zotti et al., 1997). Over-oxidizing polypyrrole increased the preferential collection of cations over anions in the polymer matrix (Sahin et al., 2008; Ugur et al., 2004). Metallocenes, bipyridines, porphyrines and other organometallic redox-active polymers were found to be interesting candidates for electrochemical separation processes, since electron-transfer rates and electronic structure of the metal-ligand system can be manipulated with an applied electric potential (Su and Hatton, 2017a). When the electrode was oxidized the metal became positively charged and thus stored ions reversibly. Several organo-metals based on for example Co (Simon et al., 1985), Os (Genborg and Sharp, 1993; Grumelli et al., 2006; Lindall et al., 1996) or Fe (Balasubramanian et al., 2002; Bruckenstein et al., 1998; Hillman et al., 1992; Jureviciute et al., 2000) have been proposed for the separation of different anions, and in some cases cations (Figure $2.7 \mathrm{c}$ ). 
$\mathbf{a}$<smiles>[3H]c1ccc(C)n1Cc1ccccc1</smiles>

b

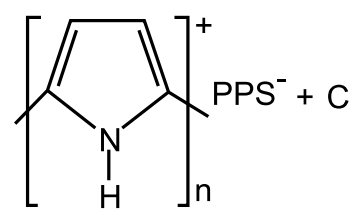

C<smiles>CC(C)(C)C1CCCCC1CC(=O)c1ccccc1F</smiles>

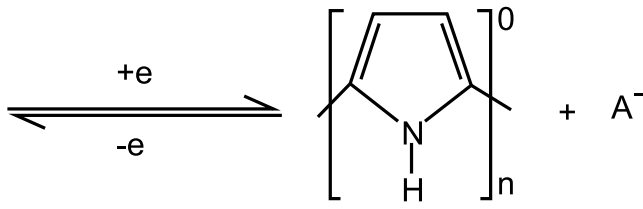

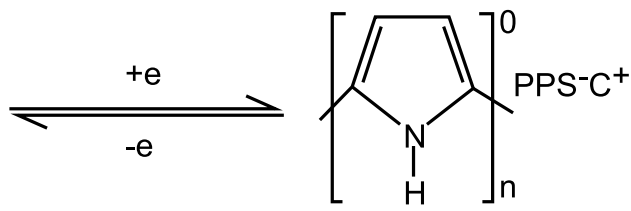

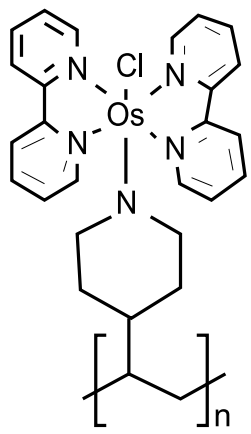

Figure 2.7. Redox reactions and ion exchange properties of polypyrrole films for a Anion (A, Cl- or CIO4-) and b Cation extraction (PPS stands for polystyrene sulfonate) (Reproduced with permission from Wu et al., 2002). c Examples of organometallics. (from left to right: Ferrocene, cobaltocene and osmium-bipyridine). (Reproduced with permission from Su and Hatton, 2017a).

Ad- and desorption of ions can also be realized by electrochemically induced $\mathrm{pH}$ changes when using $\mathrm{pH}$ responsive coatings such as poly-4-vinylpyridine (Figure 2.8 a) (Pascal et al., 2007). In an aqueous environment, water splitting can be locally induced at potentials over $1.23 \mathrm{~V}$, leading to a local change of the $\mathrm{pH}$ (Kao et al., 2008; Morimoto et al., 2008). Redox couples such as quinon/hydroquinone (Fomina et al., 2016; Simmons et al., 2000; Singh and Paul, 2015) and anilin/hydroanilin (Frasconi et al., 2010; Pavitt et al., 2017) may serve as proton source or sink, and thus influence the $\mathrm{pH}$ and their storage properties (Figure $2.8 \mathrm{~b}$ ). 
a

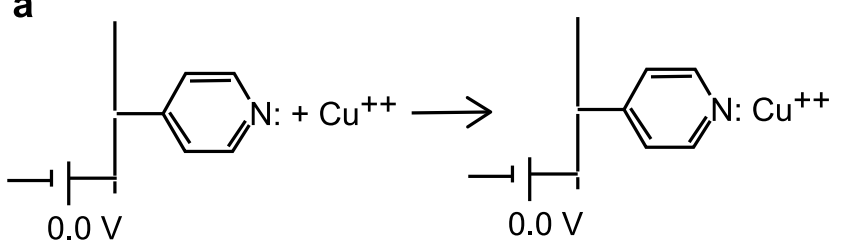

b

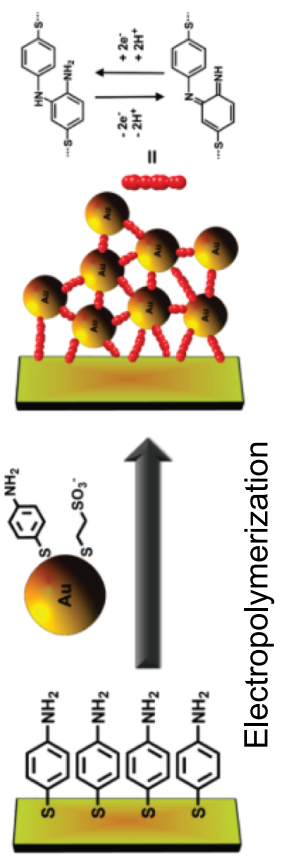

Figure 2.8. a Electro-triggering of pH-switchable ligands: (top) capture step at low or zero potential, (middle) expulsion step at electro-oxidizing potential and (bottom) spontaneous restoring at low or zero potential (Reproduced with permission from Pascal et al., 2007). b Schematic representation of electropolymerization of bis-aniline-cross-linked Au NP composite, and generation of electrochemically induced $\mathrm{pH}$ changes in aqueous solutions (Reproduced with permission from Frasconi et al., 2010).

\subsubsection{Faradic separation of larger molecules}

While most systems were developed for the separation of small ions, some of the systems presented earlier have been used for ad- and desorption of larger molecules (chemical pollutants, proteins and cells: summarized in Table 2.3). Polyvinyl(ferrocene) functionalized carbon nanotube electrodes were reversibly oxidized and reduced, enabling good separation (separation factor $>140$ ) of carboxylate, sulfonate and phosphonate (Su et al., 2016). Using the same approach, protein (lysozyme, myoglobin, horseradish peroxidase, ribonuclease-A, $\alpha$-chymotrypsin, bovine serum albumin) ad- and desorption was realized (Figure 2.9) (Su et al., 2017a).

Further, electro responsive polymers have been used, such as composites of poly(N-methyl pyrrolydinium) and poly(styrene sulfonate) onto which dopamin reversibly adsorbs at reductive potential (Miller and Zhou, 1987). The adsorption rate and adsorbed amount of glucose oxidase could be increased by applying a positive potential to a polyanion-doped polypyrrole film (Khan and Wernet, 1997). Molecular imprinting of overoxidized sulfonated polypyrrole (OSPPy) was used for the separation 
of L-glutamic acid (Figure 2.10) (Deore et al., 2000), naproxene (Ameli and Alizadeh, 2012), salicylate (Ameli and Alizadeh, 2011) and fluoroquinolones (Liu et al., 2012). Polytiophene electrodes (poly(3-dodecylthiophene) and poly(3-octylthiophene)) reversibly adsorbed neutral arsenobetaine that passively binds to the long hydrophobic allyl chains at open circuit potential, with desorption triggered by the reversible oxidation of the polymer (Tamer et al., 2003; Yates et al., 2002).
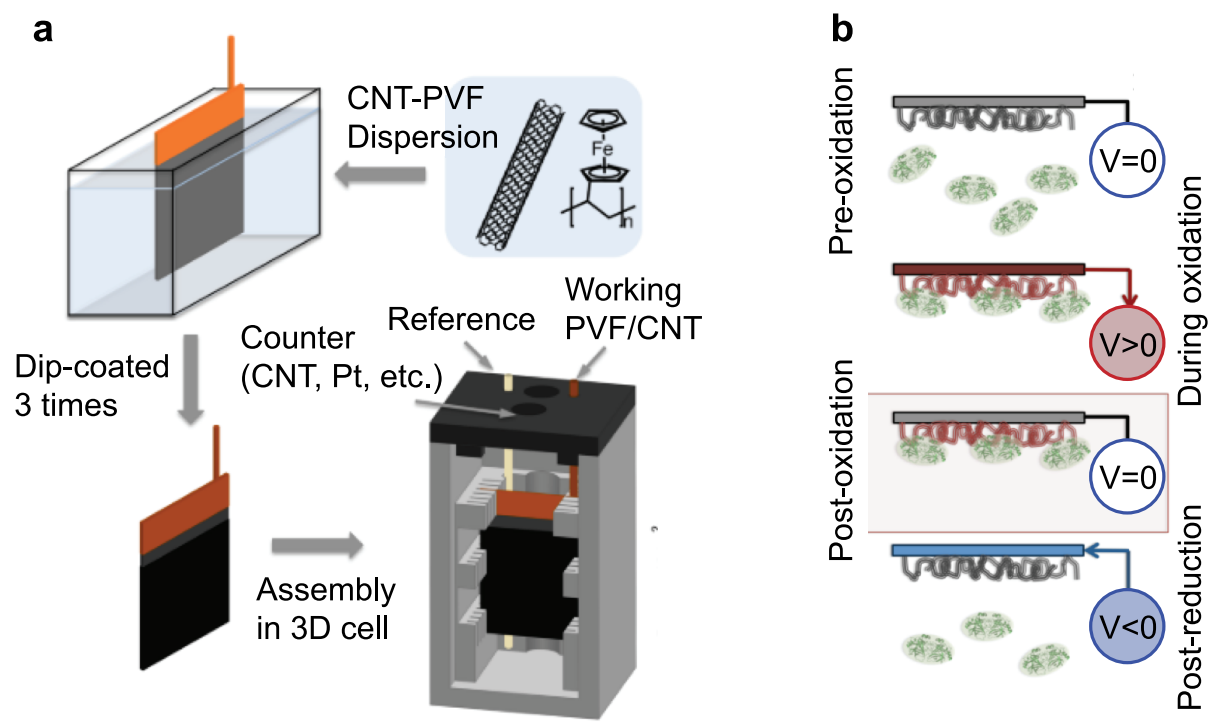

Figure 2.9. a Preparation of polyvinyl(ferrocene)/carbon-nano tube electrodes. b Schematic of protein ad- and desorption depending on applied potential (Reprodced with permission from Su et al., 2017a).

a

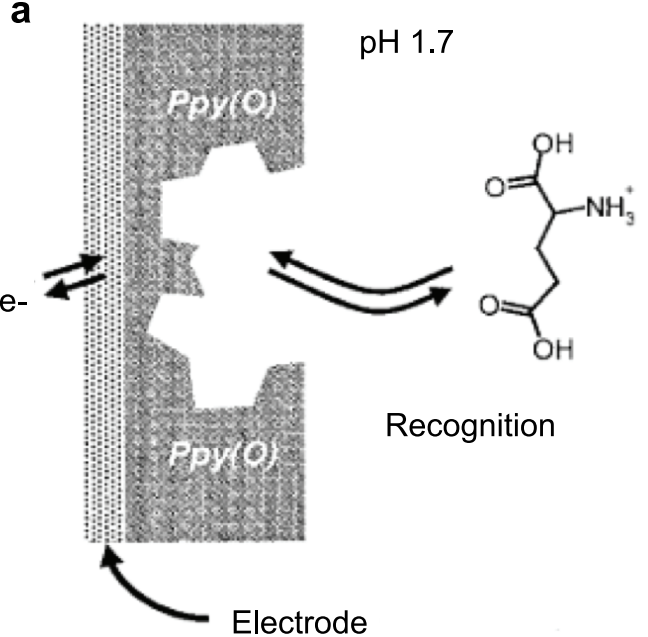

b

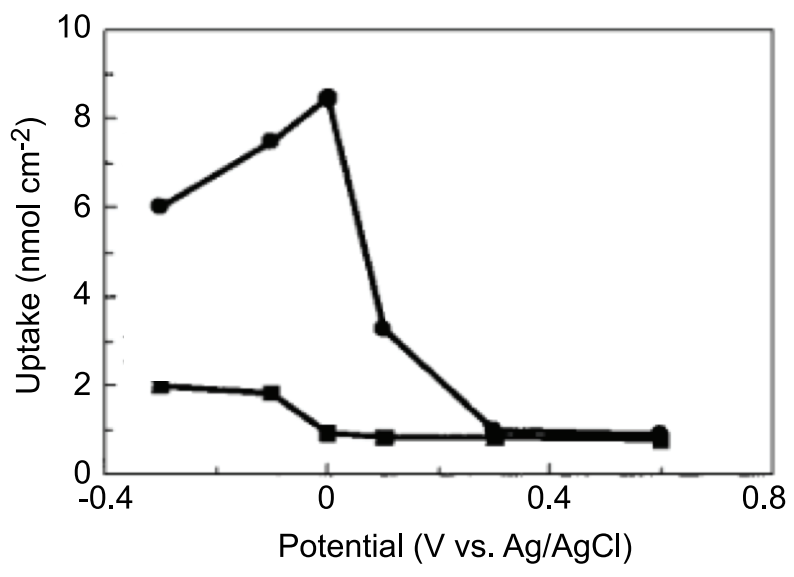

Figure 2.10. a Schematic of uptake/release of glutamic acid cation upon charging and discharging molecularly imprinted over-oxidized polypyrrole film at pH 1.7. b Effect of applied potential on the uptake of $10 \mathrm{mM} \mathrm{L-}$ and D-glutamic acid (Reproduced with permission from Deore et al., 2000). 
When targeting protein or cell separation, it is important to avoid irreversible fouling of the electrodes, and thus, antifouling coatings were developed. For example, penta(ethylene glycole) groups were used as an antifouling layer, which could be modified with ligands specific for a protein or a cell. This ligand could then be linked to a redox active quinone protionic ester (Figure 2.11). In this way, the target cell or protein could be captured by the ligand, and released upon applying a negative potential to reduce the quinone linker (Hodneland and Mrksich, 2000; Yeo et al., 2001, 2003; Yousaf et al., 2001a, 2001b). In a similar fashion, polyethylene glycol was used in combination with redox-active haeme groups for the reversible binding of $\beta$-lactoglobulin (Lam et al., 1995). Interesting reviews on these systems are (Collins and Arrigan, 2009; Su and Hatton, 2017b).

a

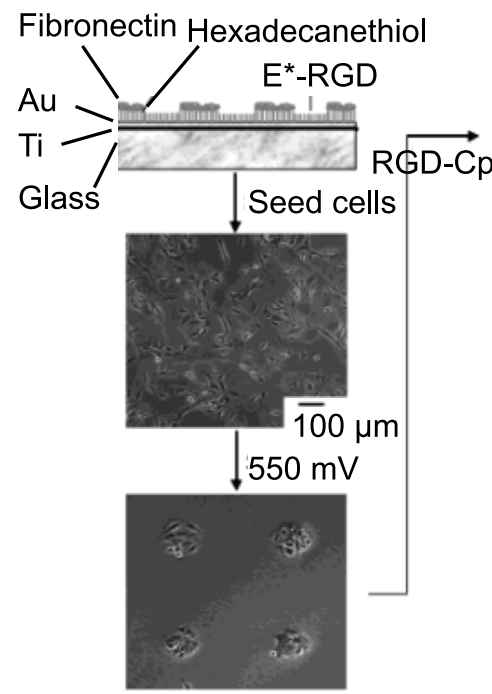

b

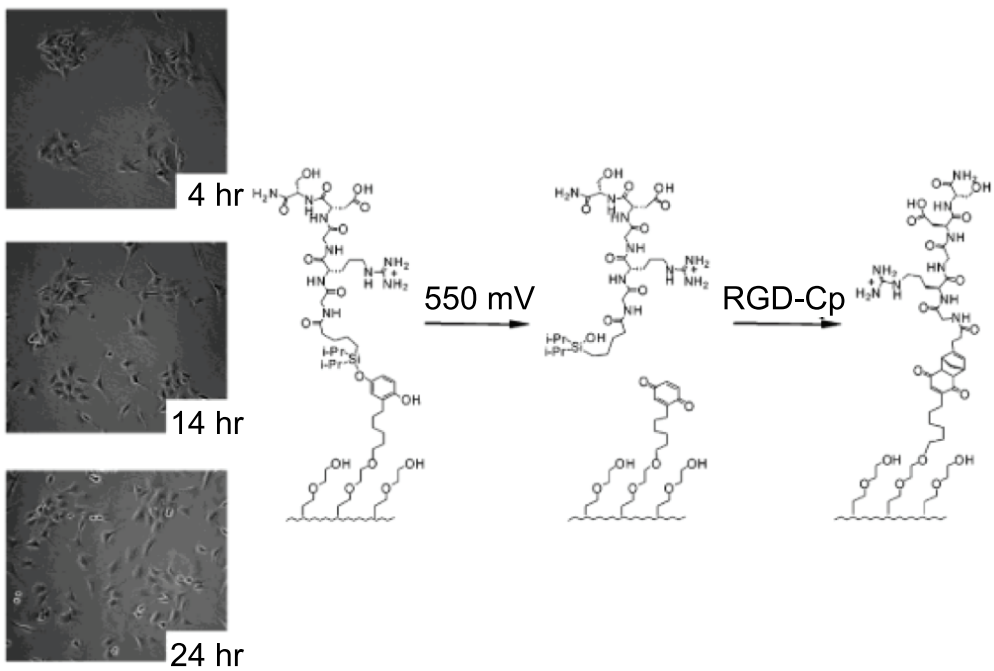

Figure 2.11. a Capture and release of fibroblast cells using a ligand that can be sacrificed when applying a potential of $550 \mathrm{mV}$ to a hydroquinone-linker. b Reaction scheme for the electro-responsive surface coating (Reproduced with permission from Yeo et al., 2003). 


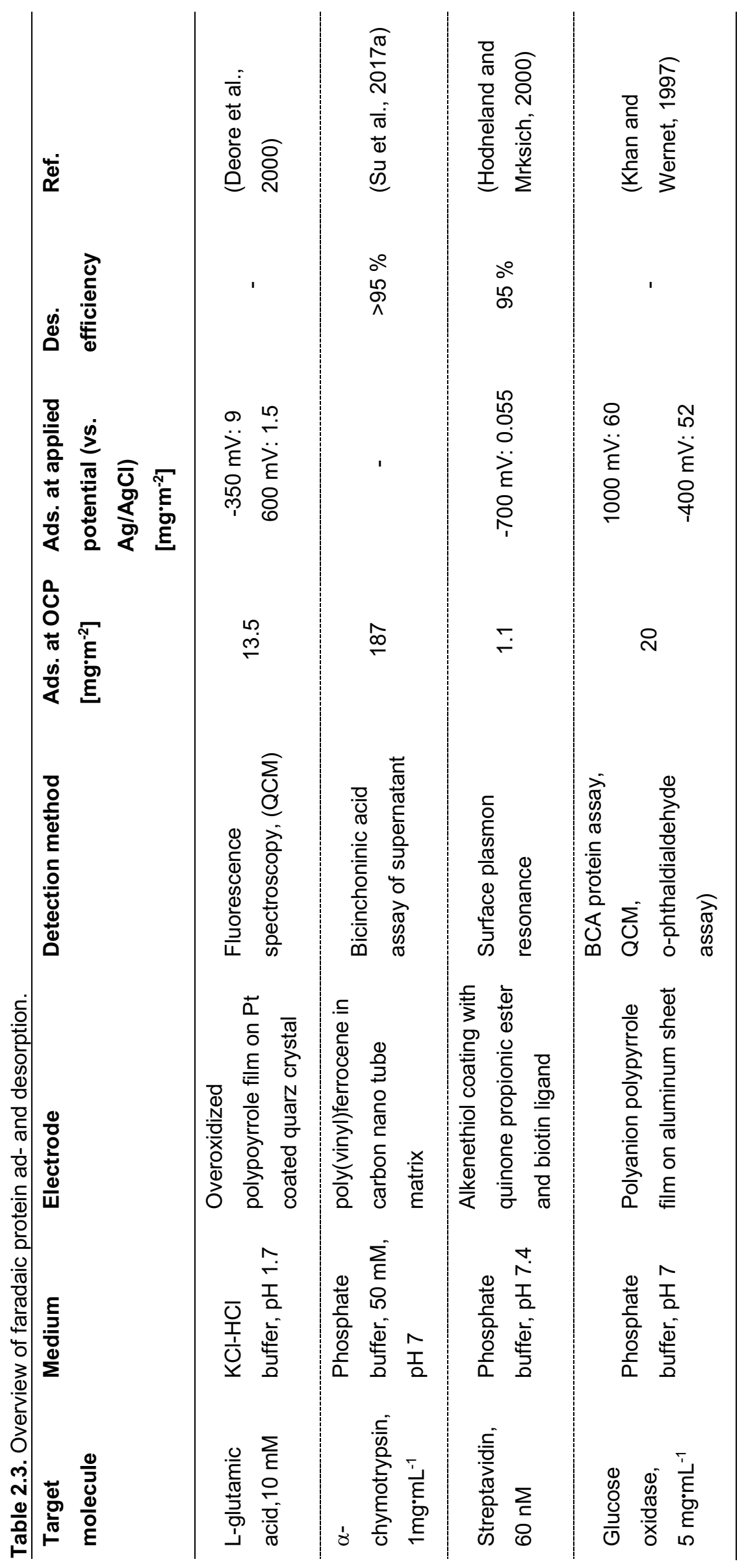




\subsection{Methods to analyze ad- and desorption or macro molecules}

To study the impact of electrode potential on ad- and desorption of macromolecules, such as proteins, different techniques were used. It was possible to indirectly measure the amount of adsorbed material through bulk fluid depletion; however one can also directly observe the amount of material adsorbed at a surface, using reflectometry or surface plasmon resonance. Further, with AFM the ad- and desorption forces may be quantitatively measured (Table 2.4). In the following section, we discuss these methods (see also Table 2.1 and 2.3 for the respective references).

\subsubsection{Indirect measurement through bulk depletion}

Adsorption and desorption of macromolecules can be measured indirectly by tracking their concentration in the bulk solution e.g. by measuring the conductivity, UV absorbance (Chapter 6), fluorescence, or by using target specific assays (e.g. bicinchoninic acid assay (Khan and Wernet, 1997; Su et al., 2017a), o-phthaldialdehyde assay (Khan and Wernet, 1997)). The sensitivity depends on the detection method, and sampling is sometimes difficult. Nevertheless, the clear advantage that these techniques hold is that they are independent of the electrode material, meaning that any type of electrode and/or coating can be tested, also on large scale.

\subsubsection{Direct surface adsorption measurement: reflectometry}

In reflectometry a laser is projected through a prism onto a planar silicon substrate, and the intensity of the reflected polarized light is measured, which can be related to the amount of adsorbed material through the refractive index. To increase the sensitivity of the method, usually a transparent silicon oxide layer is grown on top of the silicon. For electrochemically driven processes this was impractical due to the insulating properties of silica; therefore a gold coating was applied, in spite of it having a complex refractive index that reduces the signal intensity (Barten et al., 2003b; Roques-Carmes et al., 2002). Gold surfaces can be easily modified via thiol bonds and have served as a basis for diverse polymer coatings. Other potential coatings are indium tin oxide or reduced graphene oxide (Fritz et al., 2019b), since both are 
conductive and have a lower complex refractive index compared to gold. Because the laser light has to travel through the electrolyte solution, this solution should be clear and thus may only contain components at low concentration.

\subsubsection{Direct surface adsorption measurement: surface plasmon resonance} In contrast to reflectometry, in surface plasmon resonance the laser is projected onto the back side of the electrode; the intensity of the reflected light depends on the generation of surface plasmons. At a certain angle of the incoming light, all energy is converted into fluctuation of electrons at the metal-electrolyte interface and the reflection is minimal. This so called SPR-angle depends on the refractive index the interface and thus on the amount of e.g. proteins adsorbed. In SPR, mostly gold is used but also silver or graphene coated substrates have been applied (Pattnaik, 2005; Wu et al., 2017).

\subsubsection{Measuring adsorption forces: Atomic force spectroscopy}

Using atomic force spectroscopy the interaction strength between two interfaces can be determined directly, by following the deflection of a cantilever upon approaching a planar substrate. The cantilever can be equipped with a very sharp, conical, or colloidal probe, and the substrate can be an electrode (e.g. gold) in an electrochemical cell. As mentioned earlier this technique can be used to directly measure the double layer potential of an electrode (Barten et al., 2003a; Hillier et al., 1996; Chapter 7) but also other surface forces (Chapter 7). Direct interactions between single molecules and interface can be measured by modifying the probes with macromolecules. The sample preparation and data interpretation are not trivial, however.

\subsection{Conclusion}

We summarized different approaches to use capacitive and faradic currents for the separation of ions as well as larger molecules such as proteins and even whole cells. Furthermore we discussed different electrode materials ranging from porous carbon to metallic surfaces and (electro responsive) coatings, such as conductive polymers or organometallics, for this purpose. 
The overview and perspective that this review offers is important to position electrochemical separation processes that show a great potential as a next generation technology that answers today's challenges, such as improved and milder separations at reduced environmental impact. Therefore, we pointed out achievements and challenges for various species and indicated where cross-overs between the applications exist.

Table 2.4. Overview of advantages and challenges of different methods to study the impact of an applied potential on ad- and desorption of macromolecules (e.g. proteins)

\begin{tabular}{|c|c|c|}
\hline Techniques & Advantages & Challenges \\
\hline E-Reflectometry & $\begin{array}{l}\text { Direct observation } \\
\text { of changes at the } \\
\text { interface }\end{array}$ & 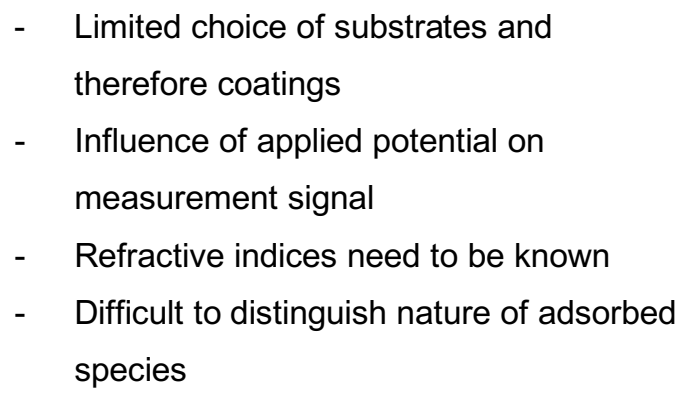 \\
\hline E-SPR & $\begin{array}{l}\text { - Direct observation } \\
\text { of changes at the } \\
\text { interface } \\
\text { - } \quad \text { Simple to use } \\
\text { - } \quad \text { High sensitivity }\end{array}$ & $\begin{array}{l}\text { - } \quad \text { Limited choice of substrates and } \\
\text { therefore coatings } \\
\text { - } \quad \text { Influence of applied potential on } \\
\text { measurement signal } \\
\text { - Difficult to distinguish nature of adsorbed } \\
\text { species }\end{array}$ \\
\hline $\begin{array}{l}\text { Assay/UV } \\
\text { detection in } \\
\text { outlet/ } \\
\text { Fluorescence in } \\
\text { outlet }\end{array}$ & $\begin{array}{l}\text { Determine the } \\
\text { change in } \\
\text { concentration of } \\
\text { specific species }\end{array}$ & $\begin{array}{ll}\text { - } & \text { Sensitivity } \\
\text { - } & \text { No information about location of protein } \\
\text { - } & \text { Sampling, impurities }\end{array}$ \\
\hline E-AFM & $\begin{array}{ll}\text { - } & \text { Information about } \\
\text { protein specific } \\
\text { surface interactions } \\
\text { - } \quad \text { Quantification of } \\
\text { interaction force }\end{array}$ & $\begin{array}{ll}\text { - } & \text { Single molecule interaction } \\
\text { - } & \text { Difficult to operate and interpret } \\
\text { - } & \text { Limited choice of electrodes }\end{array}$ \\
\hline
\end{tabular}





\section{CHAPTER 3}

\section{EXERGY ANALYSIS OF}

\section{MEMBRANE CAPACITIVE DEIONIZATION (MCDI)}

Capacitive deionization (CDI) and membrane capacitive deionization (MCDI) are widely considered as promising, highly energy efficient processes for water desalination, of which commonly used performance indicators are the average salt adsorption rate, the salt removal efficiency, and the charge efficiency. Quantification of the sustainability performance of CDI and MCDI is still scarce, and in this paper, we use exergy analysis to evaluate the resource use efficiency of membrane capacitive deionization (MCDI). The electric as well as chemical exergies of the salt solution, and the stored ions, are used to calculate the exergy efficiency $\left(\eta_{e x}\right)$ and cumulative exergy losses (CEL) ranging between 2 to $13 \%$ and 0.5 to $8 \mathrm{~J} \cdot \mathrm{mol}$ water ${ }^{-1}$, respectively. From an exergetic point of view, passive adsorption in combination with active desorption $(-0.9 \mathrm{~V})$ is favorable, yielding the highest $\eta_{e x}$ and lowest CEL values. The combination of active salt adsorption using an electric field, with either passive or active desorption gives higher productivities, but at the cost of a disproportionate amount of exergy (and energy) input.

This chapter has been published as:

P. A. Fritz, F. K. Zisopoulos, S. Verheggen, S., K. Schroën, and R. M. Boom. Exergy analysis of membrane capacitive deionization (MCDI). Desalination 444, 162-168 (2018). 


\subsection{Introduction}

The scarcity of fresh water leads to enhanced interest in sustainable and efficient water purification methods (United Nation, 2017; United Nations, 2016). Widely distributed and established purification processes such as reverse osmosis and multistage flash distillation are high in energy consumption, since a lot of water needs to be removed from the electrolytes. In reverse osmosis for example high pumping energies are required to overcome the osmotic pressure and the membrane resistance, with modern facilities having an energy requirement of 2.9 to $3.7 \mathrm{kWh} \cdot \mathrm{m}^{-3}(190-240 \mathrm{~J} \cdot \mathrm{mol}$ water-1), going up to $4 \mathrm{kWh} \cdot \mathrm{m}^{-3}\left(260 \mathrm{~J} \cdot \mathrm{mol} \mathrm{water}^{-1}\right.$ ) for multistage flash distillation (Anderson et al., 2010; Fitzsimons, 2011).

CDI technology is much less energy intensive: energy usages of 0.1 to $0.6 \mathrm{kWh} \cdot \mathrm{m}^{-3}$ (6.5 to $38.9 \mathrm{~J} \cdot \mathrm{mol}$ water $^{-1}$ ) have been reported (Anderson et al., 2010; Farmer et al., 1996a, 1996b; Welgemoed and Schutte, 2005). The process focusses on the removal of ions from water based on active electro-sorption into porous carbon electrodes; the ions being stored at the electric double layer due to chemical charges on the interface or charging of the electrodes (Porada et al., 2013). Reviews about the working principle as well as module architectures have been published (Oren, 2008; Porada et al., 2013; Suss et al., 2015). For the electrodes, different carbon sources have been discussed, such as activated carbon, 3D graphene (El-Deen et al., 2016b), hierarchical carbon (Suss et al., 2013), or carbon cloths (Han et al., 2015), that have high surface areas (up to $2500 \mathrm{~m}^{2} \cdot \mathrm{g}^{-1}$ of material) (Pandolfo and Hollenkamp, 2006).

In order to evaluate the performance of CDI, several classical characteristics have been reported such as the average salt removal rate, as well as energy arguments (Anderson et al., 2010; García-Quismondo et al., 2013; Han et al., 2015; Hemmatifar et al., 2016). To the best of our knowledge, we use exergy for the first time to evaluate the resource use efficiency of MCDI starting from our own experimental data. Exergy quantifies the useful work that can be extracted from a resource relative to an environmental reference, therewith also allowing for a detailed cross comparison with other desalination processes (Dincer, 2002).

Exergy is a thermodynamic state variable that may capture both the quantity and the quality of the resources used in a process. It describes the potential of a system to do work when it equilibrates with the environment of reference (Dincer and Rosen, 
2013; Hevert and Hevert, 1980; Rosen et al., 2008). The fundamental exergy function follows from the work of Carnot, Clausius, and Gibbs (Gibbs, 1878), and is related to the general work potential function for simple chemical systems (Equation 3.1) (Spiegler and El-Sayed, 2001).

$$
E=U+P_{0} V-T_{0} S-\sum_{i} \mu_{i} N_{i}
$$

In which $E$, stands for the exergy of the system (the maximum amount of work) with internal energy $U$, volume $V$, entropy $S$, and $N_{i}$ number of moles. $P_{0}, T_{0}$ and $\mu_{i}$ represent the pressure, temperature and chemical potential of the reference environment.

For various desalination processes (Cerci, 2002; Fitzsimons, 2011; Fitzsimons et al., 2015; Kahraman et al., 2004; Mechanics et al., 2012; Spiegler and El-Sayed, 2001 ) exergy analysis has been reported with theoretical exergy destruction values for reverse osmosis and distillation ranging between 64 - $320 \mathrm{~J} \cdot \mathrm{mol}$ water ${ }^{-1}$ (Spiegler and El-Sayed, 2001). In the current paper, we perform exergy analysis of MCDI and compare our findings not only with those made for other desalination processes, but also with conclusions drawn based on classic separation parameters, such as salt adsorption removal rate.

\subsection{Materials and Methods}

\subsubsection{Experimental work}

\subsubsection{Electrode fabrication}

Activated charcoal (acid washed with phosphoric and sulfuric acid, 10-74 $\mu \mathrm{m}$ particle size, Sigma-Aldrich, USA) was mixed with binder poly(vinylidene fluoride)

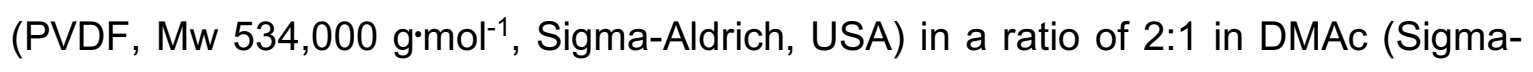
Aldrich, USA) for one hour using a mechanical stirrer. Subsequently, the carbon slurry was sonicated for one hour and cast onto a graphite sheet using a rod coater with a defined space of $200 \mu \mathrm{m}$. The thus obtained electrode sheet was dried at $70{ }^{\circ} \mathrm{C}$ for $30 \mathrm{~min}$, and ready for use. For the electrochemical characterization, and the MCDI 
experiments, the coated electrode was cut into circles with a diameter of 1 , and $5 \mathrm{~cm}$, respectively.

\subsubsection{Electrode characterization}

The cumulative pore volume as well as surface area of the electrode material after adding the binder was evaluated using nitrogen adsorption isotherms (TriStar II Plus 2.01, Micromeritics Instrument Corp., USA) based on the Brunauer-Emmett-Teller (BET) theory. The potential of zero charge was evaluated by differential capacitance minimum measurements with a potentiostat (Ivium Stat.XR, Ivium Technologies B.V., The Netherlands). As working electrode, the previously described activated carbongraphite composite with the according ion exchange membrane was used in combination with a platinum wire as counter electrode, and a silver/silver chloride $(\mathrm{Ag} / \mathrm{AgCl})$ (saturated) reference electrode. As electrolyte, $5 \mathrm{mM}$ sodium chloride $(\mathrm{NaCl})$ solution was used. The electrochemical impedance spectra were measured at a frequency of $1 \mathrm{mHz}$ with a sinusoidal voltage perturbation of $8 \mathrm{mV}$ and potential increments of $10 \mathrm{mV}$ between 0.2 and $-0.2 \mathrm{~V}$. The potential at the minimum value of capacitance was determined to be the potential of zero charge. The capacitance $C(F)$ was calculated using Equation 3.2, where Z" $(\mathrm{Ohm})$ is the imaginary part of the impedance spectra and $\omega\left(\mathrm{rad} \cdot \mathrm{s}^{-1}\right)$ the angular frequency (Wu et al., 2016a).

$$
C=\left|\frac{1}{\varpi Z^{\prime \prime}}\right|
$$

\subsubsection{MCDI experiments}

Dense cationic (molecular weight cut-off (MWCO) 60) and anionic (MWCO 180) ion exchange membranes (AMX and CMX Neospeta, Eurodia, France), as well as a spacer mesh (Meshtec NBC, Japan) were put between two of the previously described carbon electrodes. This stack was inserted into the CDI module (see Figure 3.1, left), and $5 \mathrm{mM}$ sodium chloride $(\mathrm{NaCl})$ solution was flown at a speed of $5 \mathrm{~mL} \cdot \mathrm{min}^{-1}$ in between the carbon electrodes in single pass mode. During the salt loading step, a potential of either 0 (passive), $0.9,1.2$ or $1.5 \mathrm{~V}$ (active), was applied for $300 \mathrm{~s}$ while during regeneration of the electrodes the potential was either reversed (active) or set to $0 \mathrm{~V}$ (passive) for $300 \mathrm{~s}$. The different process options are depicted in Figure 3.1 (right); the abbreviations are coded as follows: processes using active salt 
loading and unloading were termed $A A$ and depending on the potential applied $A A 0.9$, AA1.2 or AA1.5. The processes where either adsorption or desorption happens at $0 \mathrm{~V}$ (passive) were termed $\mathrm{PA}$ and $\mathrm{AP}$, respectively ( $\mathrm{A}$ for active, $\mathrm{P}$ for passive), with according numbers $(0.9,1.2,1.5)$ to indicate the applied potential in the active step.

Electrolyte flow was sustained by a pump (P-9003, ÄKTA purifier, GE Healthcare, UK), and conductivity was measured continuously in the outlet stream (UPC-900, ÄKTA purifier, GE Healthcare, UK). All experiments were conducted in triplicate, allowing no more than $1 \%$ deviation from the baseline.
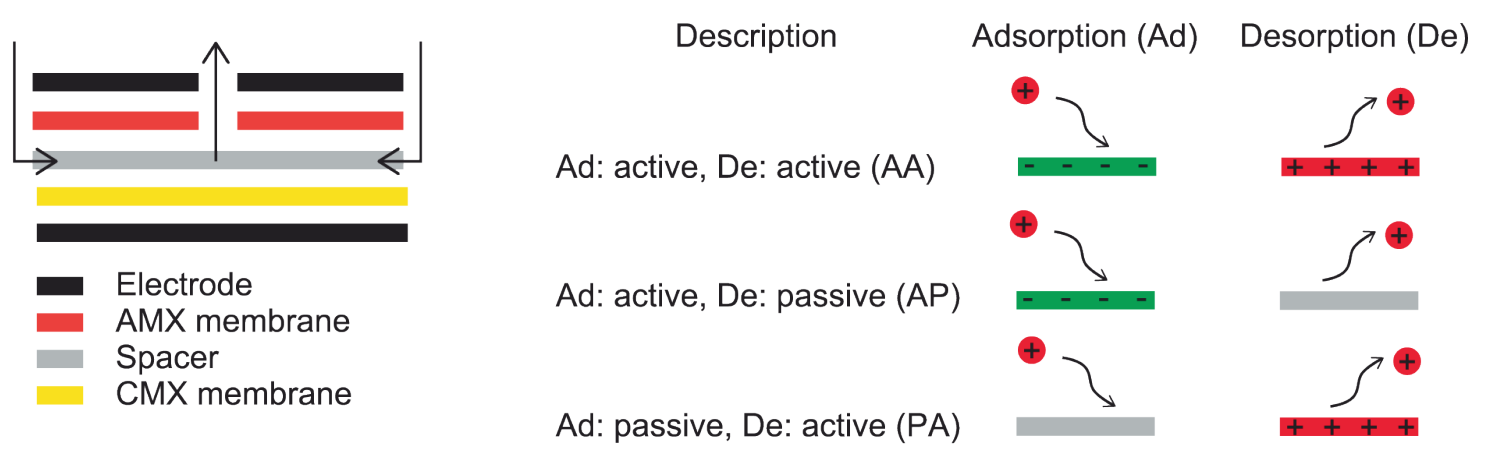

Figure 3.1. Left: schematic cross section of the MCDI module (arrows indicating electrolyte flow); Right: overview of process options, indicating electrode-cation interactions (green bar indicates negatively polarized electrode, red bar positively polarized electrode and gray bar electrode that has not been electro-chemically polarized actively).

\subsubsection{Exergy Analysis}

The dominant driving force for separation during MCDI is the electrochemical potential differences between the two electrodes. Thus the electric exergy as well as the chemical exergy related to concentration changes, will be used in this study.

The electric energy $(\mathrm{J})$ required for this process was calculated using Equation 3.3, with $E_{a p p}$ the applied potential $(\mathrm{V})$, I the current response $(\mathrm{A})$, and $t_{c y c l e}$ the potential cycle time (s).

$$
B_{e l}=E_{e l}=E_{a p p} \int_{0}^{t_{c y c l e}} I d t
$$

The chemical exergy of the electrolyte solution $B_{c h, S}(\mathrm{~J})$ was defined as the useful work obtained when brought from its restricted dead state (at thermomechanical equilibrium) to the dead state (chemical equilibrium with the reference 
environment). Here, we use the electrolyte solution model (Fitzsimons et al., 2012) (Equation 3.4) as well as the Szargut model (Equation 3.5) to calculate it. For the electrolyte solution model the reference environment was set to $293 \mathrm{~K}$, and the salt concentration of the dead state was either chosen to be equal to the incoming stream (0.3 g.L-1) or set to the salt concentration in sea water (35 g.L-1).

$$
B_{c h, s}=R T_{0}\left(n_{w} x_{w} \ln \frac{a_{w}}{a_{w, 0}}+n_{N a C l} x_{N a C l} \ln \frac{m_{N a C l} \gamma_{N a C l}}{m_{N a C l, 0} \gamma_{N a C l, 0}}\right)
$$

The subscript $w$ and $\mathrm{NaCl}$ denote water, and salt solution, respectively. $R$ is the gas constant $\left(\mathrm{J} \cdot \mathrm{mol}^{-1} \cdot \mathrm{K}^{-1}\right), T_{0}$ the reference temperature $(\mathrm{K}), n$ the number of moles $(\mathrm{mol}), x$ the mole fraction (-), $a_{w}$ the water activity $(-), m$ the molality $\left(\mathrm{mol} \cdot \mathrm{kg}^{-1}\right)$, and $\gamma$ the activity of the sodium chloride solution (-).

The chemical exergy using the Szargut model $B_{c h, S}^{S z}(\mathrm{~J})$ is described in the Equation 3.5. It includes the intrinsic chemical exergy values of water $e_{w}^{c h}$ $\left(0.9 \mathrm{~kJ} \cdot \mathrm{mol}^{-1}\right)$ and $\mathrm{NaCl}$ solution $e_{N a C l}^{c h}\left(14.0 \mathrm{~kJ} \cdot \mathrm{mol}^{-1}\right)$ (Fitzsimons et al., 2012; Szargut, 1989) since it uses the lithosphere as reference environment.

$$
B_{C h, S}^{S z}=n_{w} e_{w}^{c h}+n_{N a C l} e_{N a C l}^{c h}+R T_{0}\left[n_{w} \ln a_{w}+n_{N a C l} \ln m \gamma_{N a C l}\right]
$$

To account for non-ideal behavior of the sodium and chloride ions, the activity coefficients $\gamma_{\mathrm{NaCl}}$ and $\gamma_{\mathrm{NaCl}, 0}$ were calculated using the Pitzer equation (Equation 3.6), following (Fitzsimons et al., 2012, 2015).

$$
\begin{aligned}
\ln \gamma_{ \pm}=\quad & -\left|z_{+} z_{-}\right| A^{\varnothing}\left[\frac{\sqrt{I}}{1+b \sqrt{I}}+\frac{2}{b} \ln (1+b \sqrt{I)}]\right. \\
& +m \frac{2 v_{m} v_{x}}{v}\left\{2 \beta_{M X}^{(0)}+\frac{2 \beta_{M X}^{(1)}}{\alpha^{2} I}\left[1-\left(1+\alpha \sqrt{I}-\frac{\alpha^{2} I}{2}\right) e^{-\alpha \sqrt{I}}\right]\right\} \\
& +\frac{3 m^{2}}{2}\left[\frac{2\left(v_{m} v_{x}\right)^{3 / 2}}{v} C_{M X}^{\emptyset}\right]
\end{aligned}
$$

where $z$ is the valence of the ions $\left(\mathrm{Na}^{+}, \mathrm{Cl}^{-}\right.$); $m$ the molality of the electrolyte (moles of solute per kg of water); I the ionic strength of the solution; $A^{\varnothing}$ a constant (at $20^{\circ} \mathrm{C}$ equal 
to 0.3882 ); $b$ and $\alpha$ are fixed values which are 1.2 and 2.0, respectively. The quantities $\beta(0) M x, \beta(1) M x$, and $C_{\varnothing м x}$ are empirical parameters that are known for sodium chloride at $20{ }^{\circ} \mathrm{C}, 1$ bar, and salt concentrations ranging from 0-6 molar (Fitzsimons et al., 2012).

The chemical exergy of the capacitively stored ions on the carbon-based material was based on (Equation 3.7); adsorbed sodium $\left(\mathrm{Na}^{+}{ }_{\mathrm{ad}}\right)$ and chloride ions $\left(\mathrm{Cl}_{a d}\right)$ are released into water and form a sodium chloride solution $\left(\mathrm{NaCl}_{a q}\right)$.

$$
N a_{a d}^{+}+C l_{a d}^{-} \rightleftharpoons N a C l_{a q}
$$

In terms of exergy, this relationship was expressed with Equation 3.8, with the term on the left being the total exergy of adsorbed ions.

$$
n e_{N a^{+}, a d}^{c h}+n e_{C l^{-}, a d}^{c h}=W_{r x n}+n B_{c h, s}
$$

The work obtained $W_{r \times n}$ is equal to the electric energy $\left(0.5 q E_{a p p}\right)$ stored in the double layer; thus, we describe the chemical exergy of the adsorbed ions $B_{c h, a d}$ based on the amount of electric charges $q(\mathrm{C})$, the applied potential $E_{a p p}(\mathrm{~V})$ and chemical exergy $(\mathrm{J})$ of the equivalent amount of salt ions in solution relative to the environment (dead state) $e^{c h} \mathrm{NaCl}$, aq as shown in Equation 3.9.

$$
B_{c h, a d}=n e_{N a^{+}, a d}^{c h}+n e_{C l^{-}, a d}^{c h}=\frac{1}{2} q E_{a p p}+n B_{c h, s}
$$

To be complete, the losses in tubing and pumps e.g. kinetic exergy $B_{k i n}$ (Equation 3.10, $\sim 10^{-10} \mathrm{~J}$ ) and pressure exergy $B_{P}$ (Equation 3.11, $\sim 0.4 \mathrm{~J}$ for an over pressure of 2 bar) also need to be taken into account:

$$
\begin{gathered}
B_{k i n}=0.5 m_{e l} v_{e l} \\
B_{P}=V \Delta p
\end{gathered}
$$

With $m_{e l}$ the processed mass of electrolyte $(\mathrm{kg}), v_{e l}$ the velocity of the electrolyte $\left(\mathrm{m} \cdot \mathrm{s}^{-1}\right), V$ the volume $\left(\mathrm{m}^{3}\right)$ and $\Delta p$ the pressure difference $(\mathrm{Pa})$ between the pressure 
inside the CDI cell and the environment (101325 Pa). Since the focus of this study lays on the key driving factors of MCDI for the separation of salt from water the kinetic an pressure exergy are, however, not considered further as suggested by Spiegler and El-Sayed (2001).

\subsubsection{Process indicators}

\subsubsection{Separation performance indicators}

The separation efficiency was evaluated based on the maximum salt adsorption capacity (mSAC; $m g \cdot g^{-1}$ ), and the average salt adsorption rate (ASAR; $\mathrm{mg}^{\circ} \mathrm{g}^{-1} \cdot \mathrm{s}^{-1}$ ) (Equation 3.12). The $M S A C$ represents the amount of adsorbed salt per gram of active material on the electrodes. The latter relates mSAC to the total cycle time $t_{\text {cycle }}$ (s) (Suss et al., 2015).

$$
A S A R=\frac{m S A C}{t_{c y c l e}}
$$

\subsubsection{Exergy indicators}

To evaluate the exergetic performance of the process, two exergy indicators were used. The cumulative exergy loss CEL ( J.mol water $\left.{ }^{-1}\right)$ defined as the exergy destroyed during the process (subtracting exergy output $B_{\text {out }}$ from input $B_{\text {in, }}$ ) see Equation 3.13, with $n_{w}$ the amount of mole of water (mol).

$$
C E L=\sum \frac{B_{\text {in }}}{n_{w}}-\sum \frac{B_{\text {out }}}{n_{w}}=\sum \frac{B_{\text {destroyed }}}{n_{w}}+\sum \frac{B_{\text {wasted }}}{n_{w}}
$$

The exergy efficiency $\eta_{e x}(\%)$ was used to estimate how well exergy flows through the process, and although typically expressed as useful output exergy over total input exergy, here we consider the difference in chemical exergy between adsorption or desorption $B_{c h, \text { out }}-B_{c h, i n}$ with respect to the invested electrical exergy $B_{e l}$ (Equation 3.14).

$$
\eta_{e x}=\left(\frac{B_{c h, o u t}-B_{c h, i n}}{B_{e l}}\right) 100 \%
$$




\subsection{Results and Discussion}

The pore structure of the carbon material is of great importance for ion removal; in Figure $3.2 \mathrm{a}$, the cumulative pore volume is depicted indicating predominantly pores in the range of $50-200 \AA$. The total surface area of the electrode material is $430 \mathrm{~m}^{2}$ per $\mathrm{g}$ of electrode material, and the total pore volume $0.347 \mathrm{~cm}^{3} \cdot \mathrm{g}^{-1}$, with a micro-pore volume of $0.143 \mathrm{~cm}^{3}$ per $\mathrm{g}$ of electrode material. Due to the amphoteric character of the activated carbon (Biesheuvel, 2015) and the asymmetric set up using an anion and a cation exchange membrane, the potential of zero charge (PZC) shifts (Figure 3.2 b). Activated carbon contains oxide groups with negative charge, and thus a positive potential is required to balance all charges in the system (Gao et al., 2015b, 2016a; Wu et al., 2016b). The negative charges of the cation exchange membrane in direct contact with the cathode strengthen this effect, whereas the positive charges of the anion exchange membrane in direct contact with the anode reduce it. Thus, even with the process running at $0 \mathrm{~V}$, depolarization of the electrodes occurs.

a

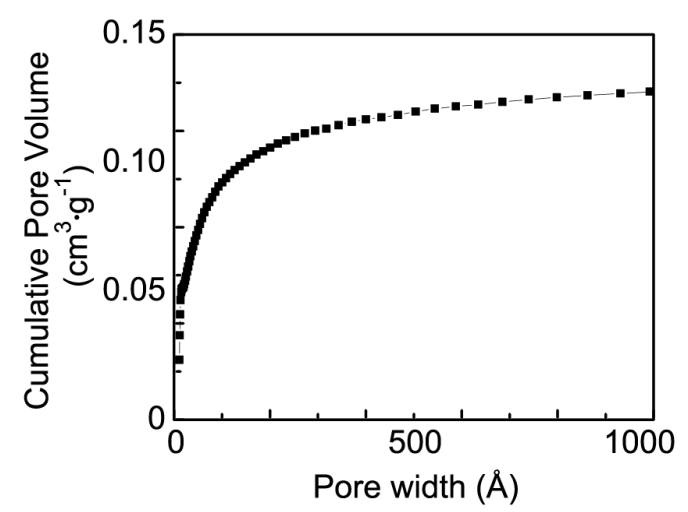

b

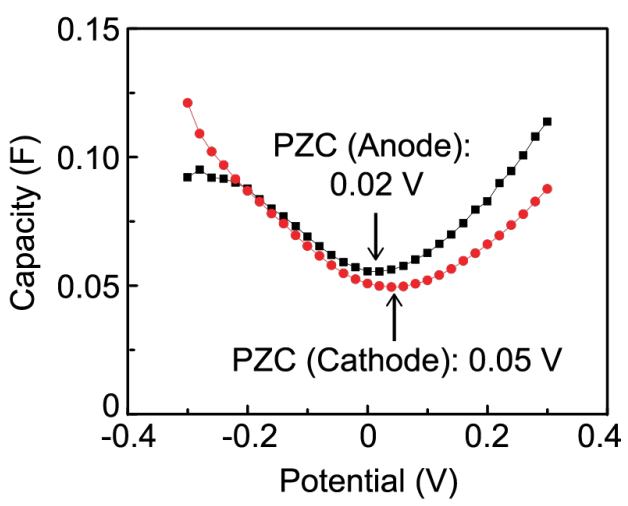

Figure 3.2. a Left: Cumulative pore volume of activated carbon electrodes as a function of pore size. b Right: Differential capacitance $(F)$ at different applied potential $(V)$; point of zero charge (PZC) is indicated by arrows.

The electrolyte solution model is used to quantify the chemical exergy. Exergy values, for a reference environment with a concentration of $0.3 \mathrm{~g} \cdot \mathrm{L}^{-1}$, are positive and are depicted for AA1.2-, AP1.2- and PA1.2-mode in Figure 3.3 a, b and c, respectively. The exergy values for the remaining experiments are summarized in Table S3.1. 
Feed water

Chemical exergy: $0.00 \mathrm{~J}$

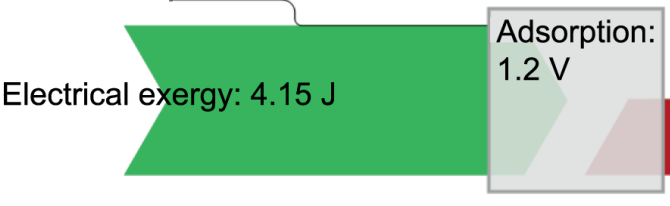

Feed water

Chemical exergy: $0.00 \mathrm{~J}$

Electrical exergy: $2.26 \mathrm{~J}$

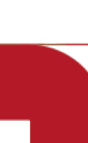

Product: Desalinated water

Chemical exergy: $0.08 \mathrm{~J}$

Electrical exergy: $0.00 \mathrm{~J}$

Adsorbed $\mathrm{NaCl}$ ions

Chemical exergy: $2.15 \mathrm{~J}$

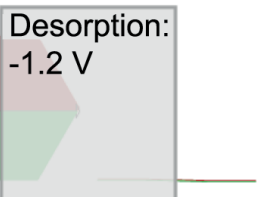

\section{Waste water}

Chemical exergy: $0.08 \mathrm{~J}$

Electrical exergy: $0.02 \mathrm{~J}$

b $\quad$ AP 1.2

\section{Feed water}

Chemical exergy: $0.00 \mathrm{~J}$

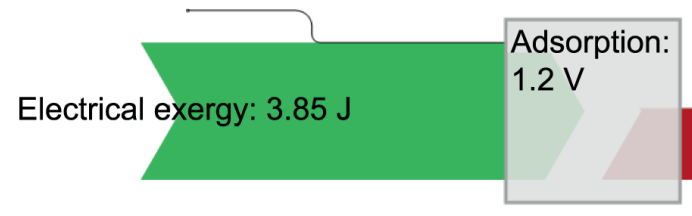

Feed water

Chemical exergy: $0.00 \mathrm{~J}$

Electrical exergy: $0.00 \mathrm{~J}$

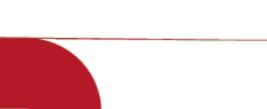

Product: Desalinated water

Chemical exergy: $0.07 \mathrm{~J}$

Electrical exergy: $0.00 \mathrm{~J}$

Adsorbed $\mathrm{NaCl}$ ions

Chemical exergy: $2.03 \mathrm{~J}$

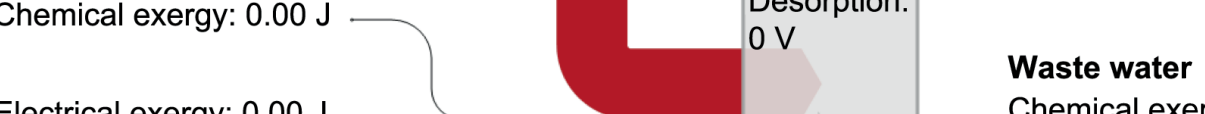

Chemical exergy: $0.07 \mathrm{~J}$

Electrical exergy: $0.00 \mathrm{~J}$

\section{c $\quad$ PA 1.2}

\section{Feed water}

Chemical exergy: $0.00 \mathrm{~J}$

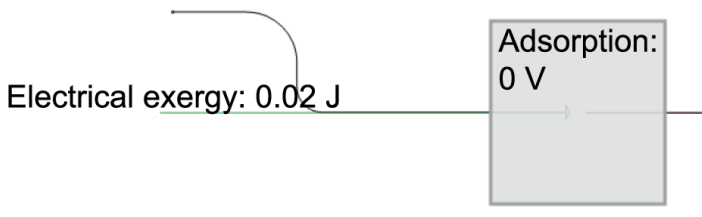

Product: Desalinated water

Chemical exergy: $0.01 \mathrm{~J}$

Electrical exergy: $0.00 \mathrm{~J}$

\section{Feed water}

Chemical exergy: $0.00 \mathrm{~J}$

Electrical exergy: $0.23 \mathrm{~J}$

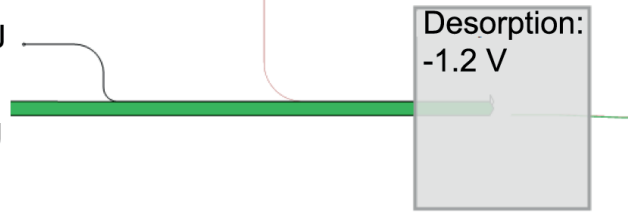

Waste water

Chemical exergy: $0.01 \mathrm{~J}$

Electrical exergy: $0.02 \mathrm{~J}$

Figure 3.3. Grassmann diagram representing exergy flows during adsorption and desorption of sodium chloride using a AA1.2-mode (1.2 V during adsorption, -1.2 V during desorption) b AP1.2-mode (1.2 V during adsorption, $0 \mathrm{~V}$ during desorption) and c PA1.2-mode ( $0 \mathrm{~V}$ during adsorption, $-1.2 \mathrm{~V}$ during desorption). The reference environment was $0.3 \mathrm{~g}^{\circ} \mathrm{L}^{-1}$. 
The calculated chemical exergy values reflect the useful work contained in the streams due to sodium chloride concentration differences with the reference environment. The feed water has the same concentration has the defined dead state $\left(0.3 \mathrm{~g}^{\cdot \mathrm{L}^{-1}}\right)$, thus the chemical exergy is $0 \mathrm{~J}$. During adsorption and desorption, the concentration is deviating to lower (e.g. $0.25 \mathrm{~g}^{\cdot \mathrm{L}^{-1}}$ for AA1.2-mode) and higher (e.g. $0.35 \mathrm{~g} \cdot \mathrm{L}^{-1}$ for $\mathrm{AA} 1.2-$ mode) values, respectively and thus positive chemical exergy values are obtained.

The magnitude of the change in chemical exergy during adsorption and desorption per mole of water separated depends on the process mode as shown in Figure 3.4 a and c. The chemical exergy change is lowest for adsorption at $\mathrm{OV}$ and highest for active adsorption at $1.5 \mathrm{~V}$ (AA1.5 and AP1.5). This is expected, since in PA mode, ions spontaneously adsorb through electrostatic interactions with the charged groups on the surface of the electrode. Ion separation in AP-mode is due to the electric charge induced by the applied potential (assuming electrostatically bound ions are not removed), while in AA-mode both types of ion interactions occur. These differences are also the reason why only AA and AP show a dependency on the maximum applied potential during adsorption, leading to the largest gain in chemical exergy of $0.07 \mathrm{~J} \cdot \mathrm{mol}$ water ${ }^{-1}$, corresponding to $1.3 \mathrm{mg}$ of adsorbed sodium chloride (Figure S3.2).

In line with the chemical exergy change, also the total electric exergy input increases with values ranging between 0.02 and $5.4 \mathrm{~J} \cdot \mathrm{mol}^{-1}$ water ${ }^{-1}$ during the adsorption step (Figure $3.4 \mathrm{~b}$ ). During desorption no electric exergy input is given for AP but between 0.16 and $3.83 \mathrm{~J} \cdot \mathrm{mol}$ water $^{-1}$ are invested for AA- and PA-mode, respectively (Figure $3.4 \mathrm{~d}$ ). Figure 3.4 e reveals that the highest average exergy efficiency over both ad- and desorption returns values of $\sim 13 \%$ and is achieved in PA-mode using $0.9 \mathrm{~V}$ during desorption; the lowest values ( 2\%) correspond to modes that use $1.5 \mathrm{~V}$ during adsorption. The values determined using the electrolyte solution model using a reference environment of sea water as well as Szargut model using the lithosphere as reference show similar results for chemical exergy change as well as exergy efficiency (Figure S3.3).

To put the results further in perspective, regular CDI operation (without membranes) is reported to achieve lower salt separation at higher energy requirement (Zhao et al., 2012a), and thus lower exergy efficiency. As an alternative to the constant potential experiments reported in the current paper, constant current operation can be 
considered. It has been shown that higher salt separation at lower energy investment (Zhao et al., 2012a) can be achieved, leading to higher exergy efficiencies.

a

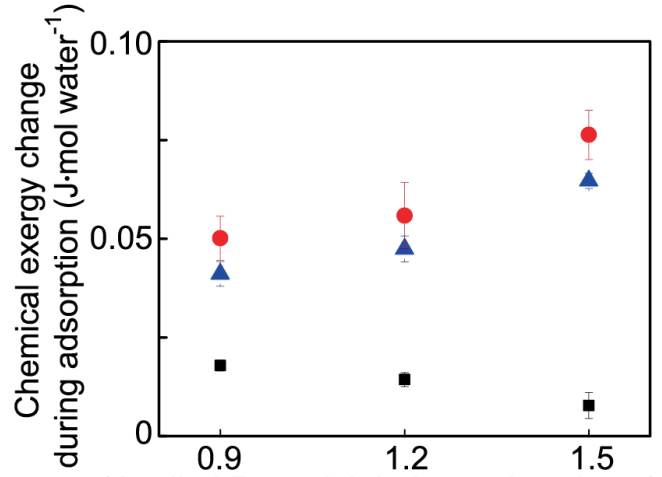

|Applied Potential during active phase| (V)

c

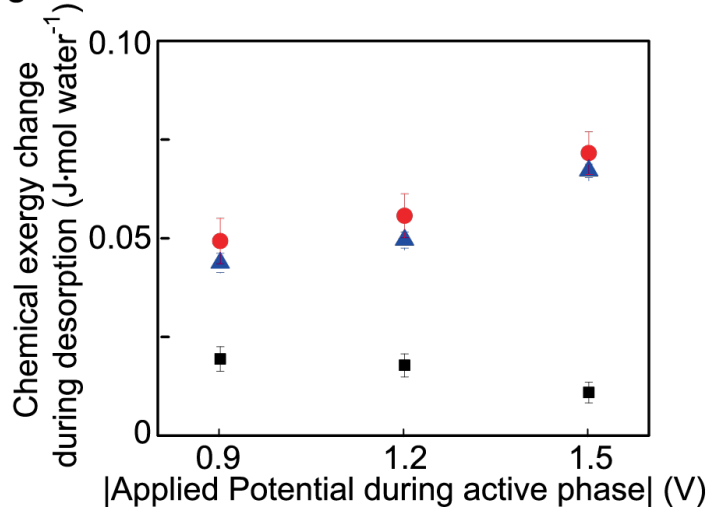

b

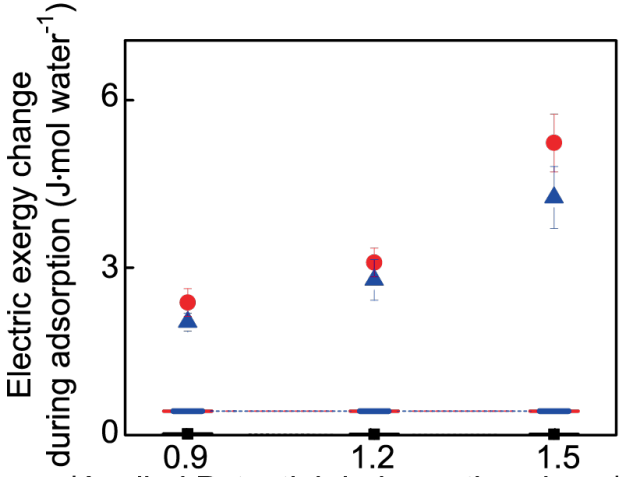

|Applied Potential during active phase| (V)

d

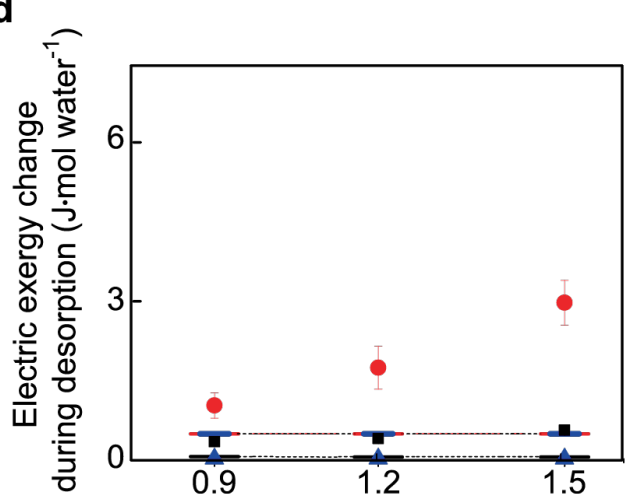

|Applied Potential during active phase| (V)

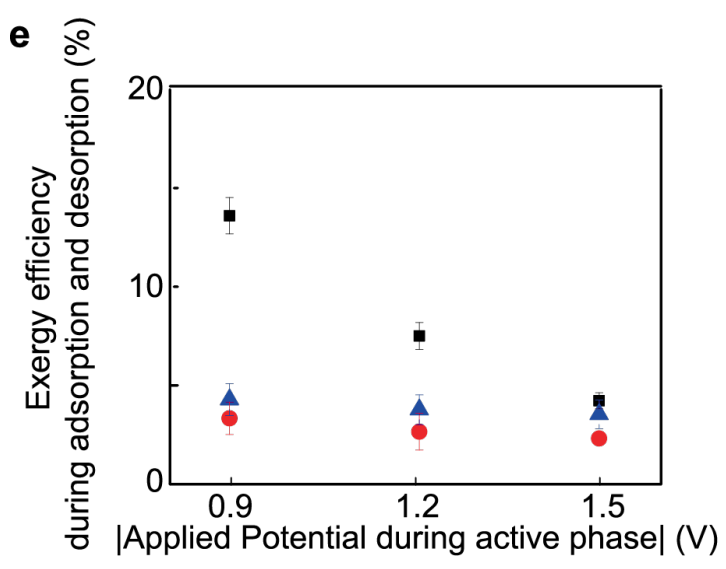

Figure 3.4. Total chemical exergy change $\left(\mathrm{J} \cdot \mathrm{mol}^{\text {water }}{ }^{-1}\right)$ during adsorption (Figure a) and desorption (Figure c). Total electric exergy input $\left(\mathrm{J} \cdot \mathrm{mol}^{\text {water }}{ }^{-1}\right)$ during adsorption (Figure b) and desorption (Figure d). Exergy efficiency of the process (\%) (Figure e). Experimental values for PA (black squares), AP (blue tringles), and AA (red circles), theoretical maximum values are indicated by horizontal lines in b and $\mathbf{d}$. Please note that the values are plotted against the absolute potential applied during the active phase; during a passive phase the applied potential is always $0 \mathrm{~V}$. 
To put these values into a theoretical framework, we consider the maximum chemical exergy describing the complete desalination of a salt solution containing $0.3 \mathrm{~g}^{\cdot \mathrm{L}^{-1}} \mathrm{NaCl}$, which is $0.44 \mathrm{~J} \cdot \mathrm{mol}$ water ${ }^{-1}$. In an ideal system with $100 \%$ exergy efficiency this would correspond to the maximum electric exergy input required. During active adsorption (AP- and AA-mode) the electric exergy is exceeding this value, meaning that more electric exergy (as high as $5.4 \mathrm{~J} \cdot \mathrm{mol}$ water ${ }^{-1}$ ) is put into the system then theoretically required for complete salt removal (Figure 3.4 b). During passive adsorption the electric exergy input is lower than $0.44 \mathrm{~J} \cdot \mathrm{mol}$ water ${ }^{-1}$ and matches with $0.04 \mathrm{~J} \cdot \mathrm{mol}$ water ${ }^{-1}$ the chemical exergy change. However, during desorption also in PA-mode the theoretical maximum value is exceeded (Figure $\mathbf{3 . 4} \mathrm{d}$ ).

When the cumulative exergy loss (CEL) is plotted versus the average salt adsorption rate (ASAR), the PA processes cluster at low ASAR and low CEL values, whereas the AA and AP processes are positioned at high CEL and high ASAR values (Figure 3.5). The relation is clearly nonlinear; the PA-mode combines a very low CEL with a moderate ASAR and thus can be qualified as a very efficient mode of operation. A higher adsorption rate can only be gained at much larger CEL, and this is what both AP and especially the AA-mode show: high and fast sodium chloride separation at the cost of a disproportionally large exergetic input.

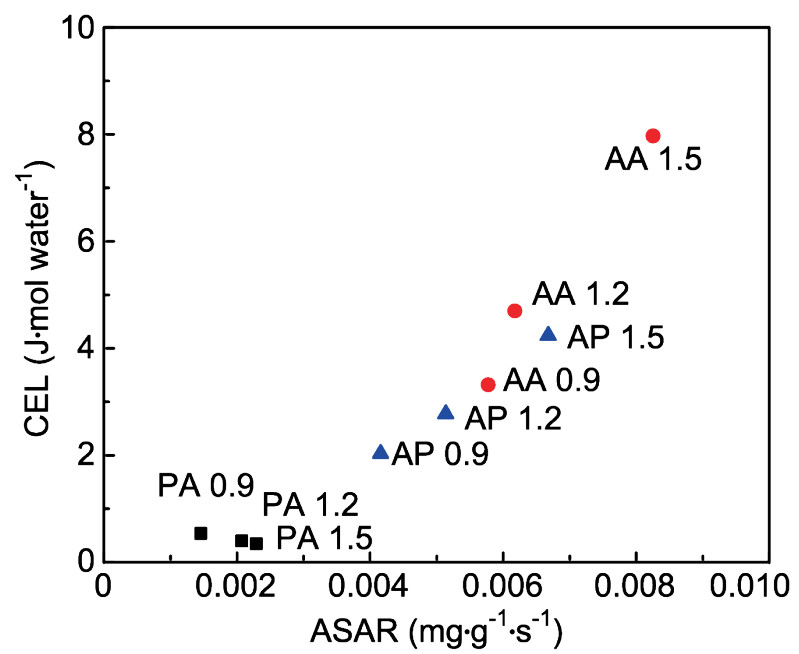

Figure 3.5. Cumulative exergy losses determined for the experimental data versus average salt adsorption rate for the different operation modes: PA in black squares, AP in blue tringles, and AA in red circles.

This insight opens up two strategies to improve the process: in PA-mode, the surface charge needs to be as high as possible to increase the salt adsorption rate, 
whereas for AA and AP-mode, lower potentials (even below $0.9 \mathrm{~V}$ ) may be used to reduce the electric exergy input. Besides, by using energy recovery systems, up to $20 \%$ of the invested energy could be stored in a super-capacitor (Długołęcki and Wal, 2013; Kang et al., 2016), leading to a reduction in CEL.

Furthermore, the exergy analysis indicates the great potential of the PA mode with high exergy efficiencies and low CEL values. This leads us to concluded that increasing the surface charge by modification with e.g. polymers would be an interesting option to increase the capacity. This feature could be especially interesting to selectively remove impurities from large feed streams, when tailoring the chemical surface charges.

These options directly follow from the exergy indicators therewith showing their usefulness for comparing MCDI with other desalination technologies. During reversed osmosis, water is desalinated by pressing it through a semipermeable membrane, while ions remain in the retentate. For brackish water a commonly applied pressure is 15 bar (Fritzmann et al., 2007) leading to a much higher exergy input $B_{P}$ of $25.2 \mathrm{~J} \cdot \mathrm{mol}$ water ${ }^{-1}$ (Equation 3.11) with $\Delta p$ being the pressure difference between the applied pressure and the environmental pressure, that is lost throughout the process when a perfect semipermeable membrane is assumed. For the desalination of sea water using reversed osmosis with an applied pressure of 60 bar (Fritzmann et al., 2007) an exergy input of $106.2 \mathrm{~J} \cdot \mathrm{mol}^{\text {water }}{ }^{-1}$ is lost. After extrapolating our analysis to the purification of sea water, a chemical exergy change of $44.5 \mathrm{~J} \cdot \mathrm{mol}^{\text {water }}{ }^{-1}$ during adsorption has to be achieved as well as during desorption. Since for AP mode no electric exergy is invested during desorption and for PA-mode the electric exergy invested during adsorption is recovered during desorption, $44.5 \mathrm{~J} \cdot \mathrm{mol}^{-1}$ represents the minimal electric exergy input required for sea water desalination (3.5 wt\%). For AAmode this value increases to $89 \mathrm{~J} \cdot \mathrm{mol}^{-1}$ since both ad- and desorption are active. These values are representing the thermodynamic limiting case for the different MCDI modes but they will increase due to exergy inefficiencies as reported above.

The values reported here, clearly show that MCDI has a potential for exergy efficient water desalination, and in future studies amongst others the electrode material will be investigated further in order to make better use of spontaneous adsorption processes that are beneficial from an exergy view point. 


\subsection{Conclusions}

Exergy was used to analyze different process modes within the MCDI process family. A process that uses passive adsorption and active desorption, is more efficient even though the typical capacity is relatively lower than that of other modes. Modes that combine active adsorption (with an electric field), with active or passive desorption, both show larger productivities, albeit at the cost of a disproportionally higher use of exergy. In comparison to reversed osmosis, the minimal CEL values for AP and AA mode are comparable but the real values are still higher due to exergy inefficiencies in the current system, which is not optimized. The exergy analysis gives guidelines on how to improve the different process modes, since it allows for direct comparison of the achieved chemical exergy change from feed stream to product and the driving force (electric exergy). It revealed the great potential of PA, which can be enhanced further by increasing the difference in the potential of zero charge between the two electrodes by incorporating additional chemical surface charges. AA and AP can only be improved by lowering electric exergy losses or increasing chemical exergy changes. 


\subsection{Supporting Information}
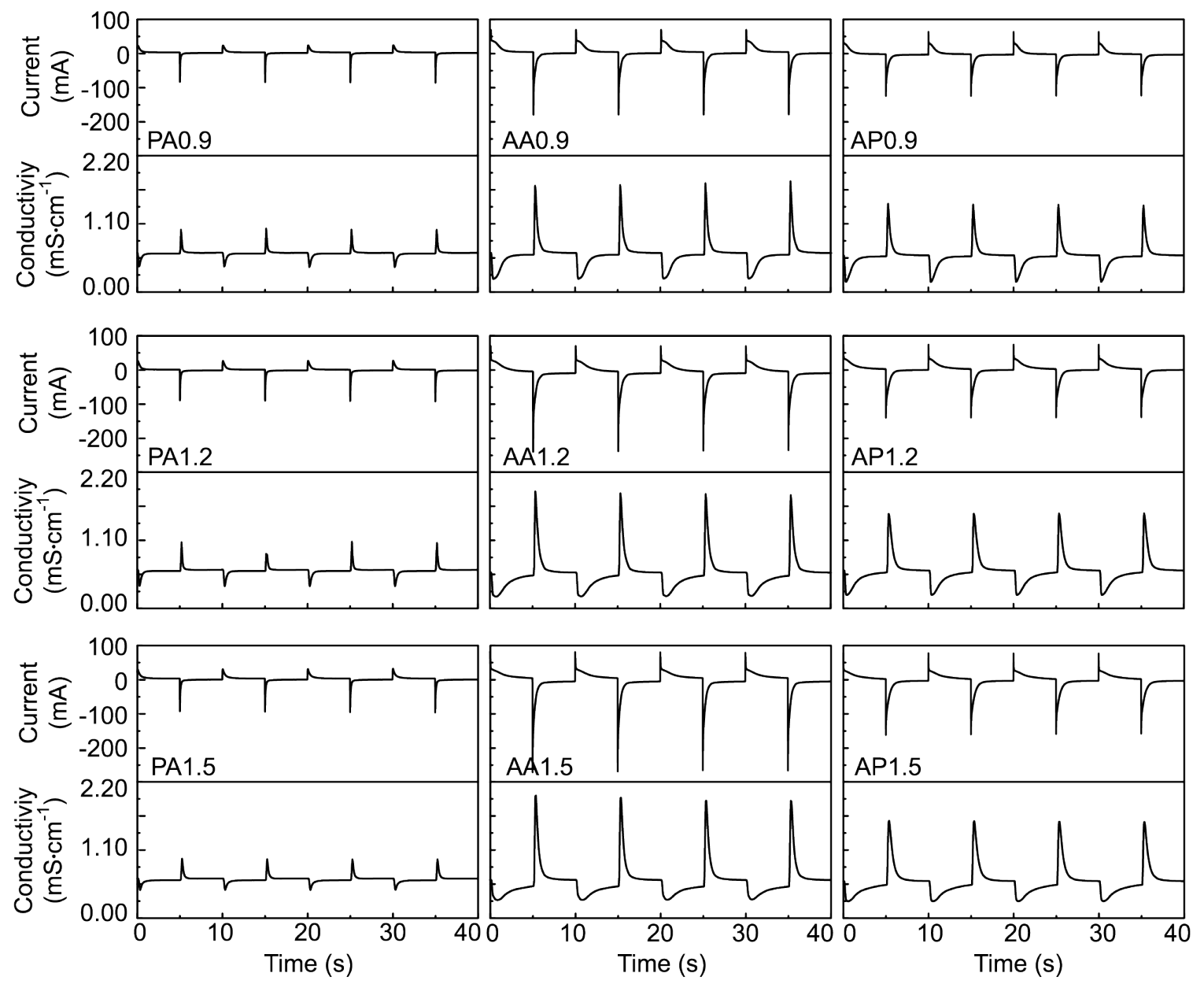

Figure S3.1. CDI performance for the individual potential patterns (PA, AA and AP) and the different applied potentials $0.9,1.2$ and $1.5 \mathrm{~V}$. Top line in the figures displays the current response and bottom line the conductivity in the outlet flow. 


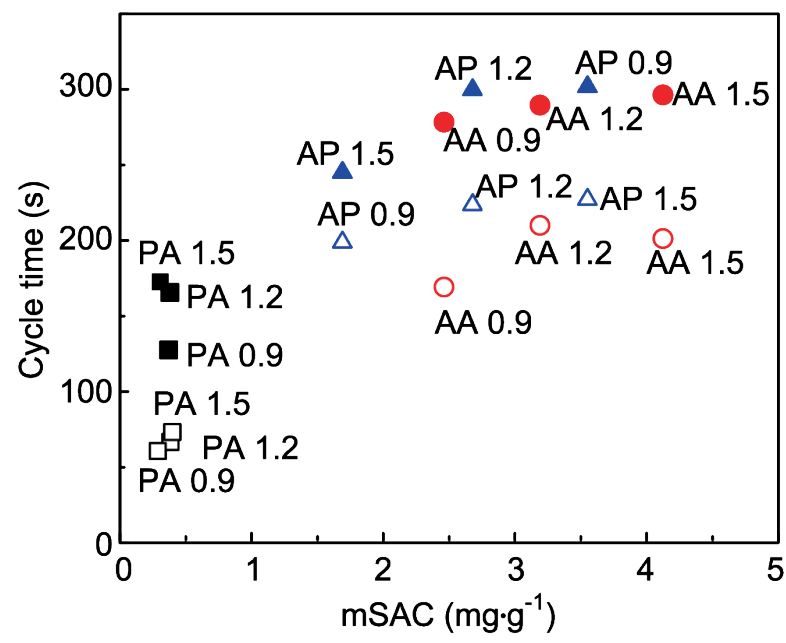

Figure S3.2. Cycle time (s) with respect to maximum salt adsorption capacity $\mathrm{mSAC}\left(\mathrm{mg}^{\cdot} \mathrm{g}^{-1}\right)$ for PA in black squares, AP in blue tringles and AA in red circles. Values for adsorption cycle are indicated with filled symbols and desorption cycle with empty cycles.

Table S3.1. Average calculated exergy content of streams for each potential pattern.

\begin{tabular}{|c|c|c|c|c|c|}
\hline Abbreviation & $\begin{array}{l}\text { Adsorption } \\
\text { potential } \\
\text { (V) }\end{array}$ & $\begin{array}{l}\text { Desorption } \\
\text { potential } \\
\text { (V) }\end{array}$ & Stream & $\begin{array}{l}\text { Electrical } \\
\text { exergy (J) }\end{array}$ & $\begin{array}{c}\text { Chemical exergy } \\
\text { (Electrolyte } \\
\text { solution model, J) }\end{array}$ \\
\hline PA0.9 & 0 & -0.9 & Adsorption in & 0.02 & 0.00 \\
\hline PA0.9 & 0 & -0.9 & Adsorbed ions & & 0.02 \\
\hline PA0.9 & 0 & -0.9 & $\begin{array}{l}\text { Purified } \\
\text { stream }\end{array}$ & 0.00 & 0.01 \\
\hline PA0.9 & 0 & -0.9 & Desorption in & 0.15 & 0.00 \\
\hline PA0.9 & 0 & -0.9 & Waste stream & 0.02 & 0.01 \\
\hline AA0.9 & 0.9 & -0.9 & Adsorption in & 3.02 & 0.00 \\
\hline AA0.9 & 0.9 & -0.9 & Adsorbed ions & & 1.57 \\
\hline AA0.9 & 0.9 & -0.9 & $\begin{array}{l}\text { Purified } \\
\text { stream }\end{array}$ & 0.00 & 0.06 \\
\hline AA0.9 & 0.9 & -0.9 & Desorption in & 1.20 & 0.00 \\
\hline AA0.9 & 0.9 & -0.9 & Waste stream & 0.02 & 0.06 \\
\hline AP0.9 & 0.9 & 0 & Adsorption in & 2.22 & 0.00 \\
\hline AP0.9 & 0.9 & 0 & Adsorbed ions & & 1.19 \\
\hline AP0.9 & 0.9 & 0 & $\begin{array}{l}\text { Purified } \\
\text { stream }\end{array}$ & 0.00 & 0.04 \\
\hline AP0.9 & 0.9 & 0 & Desorption in & 0.00 & 0.00 \\
\hline AP0.9 & 0.9 & 0 & Waste stream & 0.00 & 0.05 \\
\hline PA1.2 & 0 & -1.2 & Adsorption in & 0.02 & 0.00 \\
\hline PA1.2 & 0 & -1.2 & Adsorbed ions & & 0.02 \\
\hline
\end{tabular}




\begin{tabular}{|c|c|c|c|c|c|}
\hline Abbreviation & $\begin{array}{l}\text { Adsorption } \\
\text { potential } \\
\text { (V) }\end{array}$ & $\begin{array}{l}\text { Desorption } \\
\text { potential } \\
\text { (V) }\end{array}$ & Stream & $\begin{array}{l}\text { Electrical } \\
\text { exergy (J) }\end{array}$ & $\begin{array}{l}\text { Chemical exergy } \\
\text { (Electrolyte } \\
\text { solution model, J) }\end{array}$ \\
\hline PA1.2 & 0 & -1.2 & $\begin{array}{l}\text { Purified } \\
\text { stream }\end{array}$ & 0.00 & 0.01 \\
\hline PA1.2 & 0 & -1.2 & Desorption in & 0.23 & 0.00 \\
\hline $\mathrm{PA} 1.2$ & 0 & -1.2 & Waste stream & 0.02 & 0.01 \\
\hline $\mathrm{AA} 1.2$ & 1.2 & -1.2 & Adsorption in & 4.15 & 0.00 \\
\hline $\mathrm{AA} 1.2$ & 1.2 & -1.2 & Adsorbed ions & & 2.15 \\
\hline $\mathrm{AA} 1.2$ & 1.2 & -1.2 & $\begin{array}{l}\text { Purified } \\
\text { stream }\end{array}$ & 0.00 & 0.08 \\
\hline $\mathrm{AA} 1.2$ & 1.2 & -1.2 & Desorption in & 2.26 & 0.00 \\
\hline AA1.2 & 1.2 & -1.2 & Waste stream & 0.02 & 0.08 \\
\hline $\mathrm{AP} 1.2$ & 1.2 & 0 & Adsorption in & 3.85 & 0.00 \\
\hline $\mathrm{AP} 1.2$ & 1.2 & 0 & Adsorbed ions & & 2.03 \\
\hline AP 1.2 & 1.2 & 0 & $\begin{array}{l}\text { Purified } \\
\text { stream }\end{array}$ & 0.00 & 0.07 \\
\hline AP1.2 & 1.2 & 0 & Desorption in & 0.00 & 0.00 \\
\hline $\mathrm{AP} 1.2$ & 1.2 & 0 & Waste stream & 0.00 & 0.07 \\
\hline PA1.5 & 0 & -1.5 & Adsorption in & 0.02 & 0.00 \\
\hline PA 1.5 & 0 & -1.5 & Adsorbed ions & & 0.02 \\
\hline PA1.5 & 0 & -1.5 & $\begin{array}{l}\text { Purified } \\
\text { stream }\end{array}$ & 0.00 & 0.01 \\
\hline PA1.5 & 0 & -1.5 & Desorption in & 0.38 & 0.00 \\
\hline PA1.5 & 0 & -1.5 & Waste stream & 0.02 & 0.01 \\
\hline AA1.5 & 1.5 & -1.5 & Adsorption in & 7.15 & 0.00 \\
\hline AA1.5 & 1.5 & -1.5 & Adsorbed ions & & 3.67 \\
\hline AA1.5 & 1.5 & -1.5 & $\begin{array}{l}\text { Purified } \\
\text { stream }\end{array}$ & 0.00 & 0.10 \\
\hline $\mathrm{AA} 1.5$ & 1.5 & -1.5 & Desorption in & 3.83 & 0.00 \\
\hline AA1.5 & 1.5 & -1.5 & Waste stream & 0.02 & 0.10 \\
\hline AP1.5 & 1.5 & 0 & Adsorption in & 5.92 & 0.00 \\
\hline AP1.5 & 1.5 & 0 & Adsorbed ions & & 3.10 \\
\hline AP1.5 & 1.5 & 0 & $\begin{array}{l}\text { Purified } \\
\text { stream }\end{array}$ & 0.00 & 0.09 \\
\hline AP1.5 & 1.5 & 0 & Desorption in & 0.00 & 0.00 \\
\hline AP 1.5 & 1.5 & 0 & Waste stream & 0.00 & 0.09 \\
\hline
\end{tabular}


a

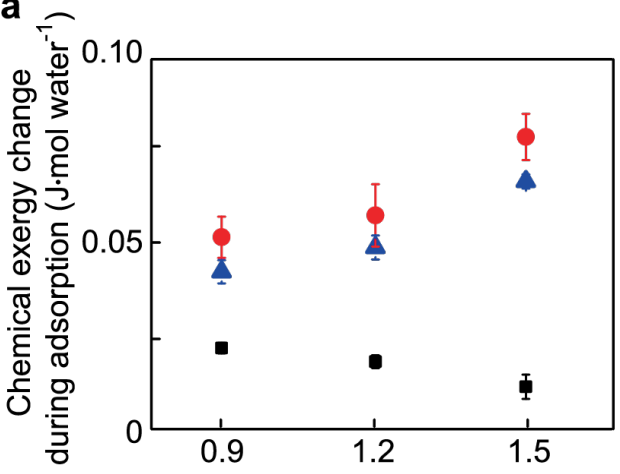

|Applied Potential during active phase| (V)
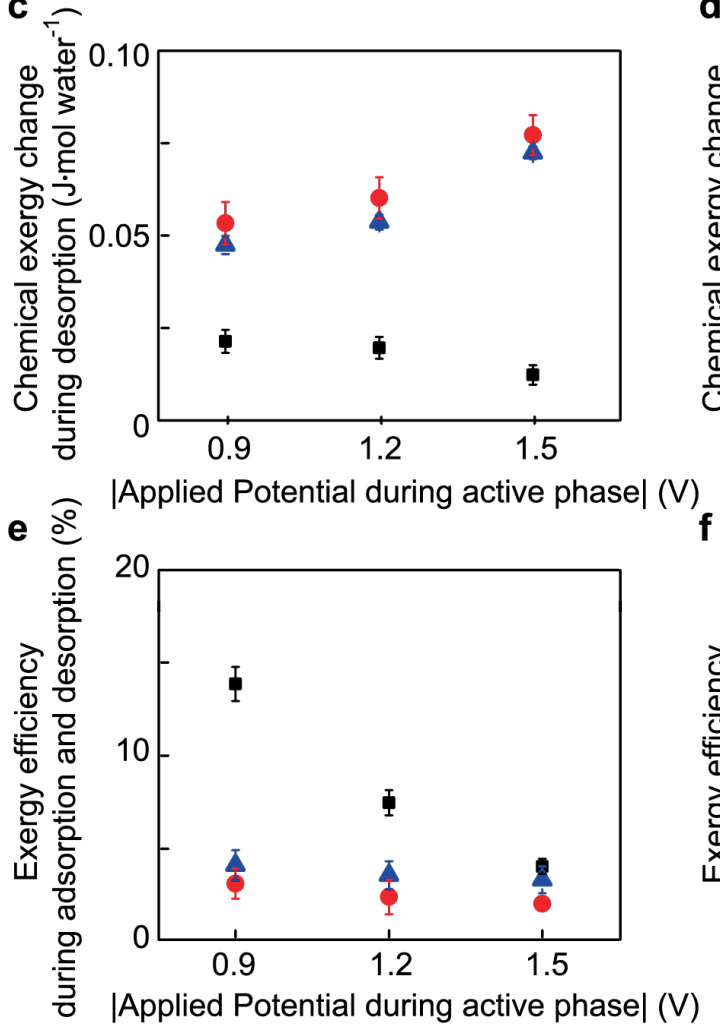

g

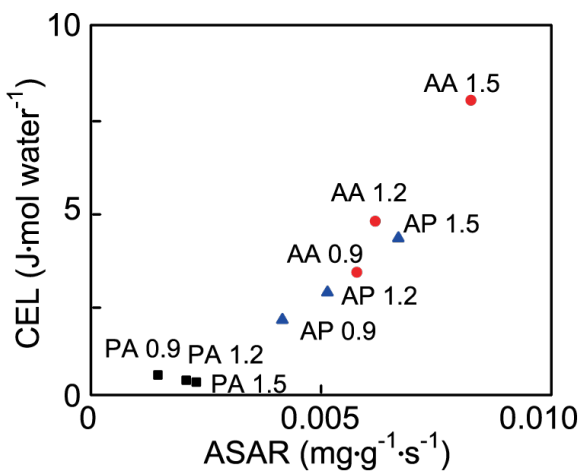

b

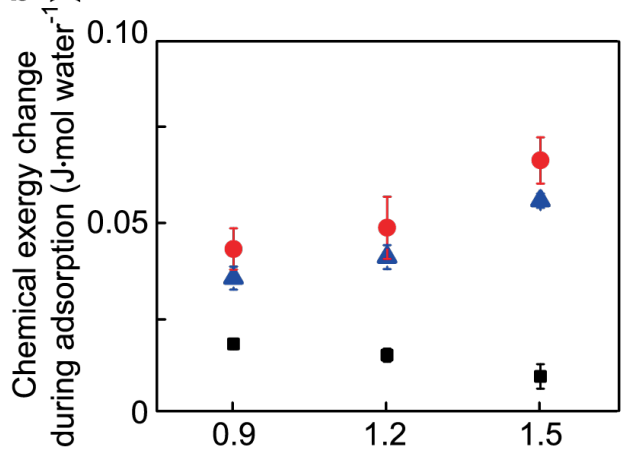

|Applied Potential during active phase| (V) d

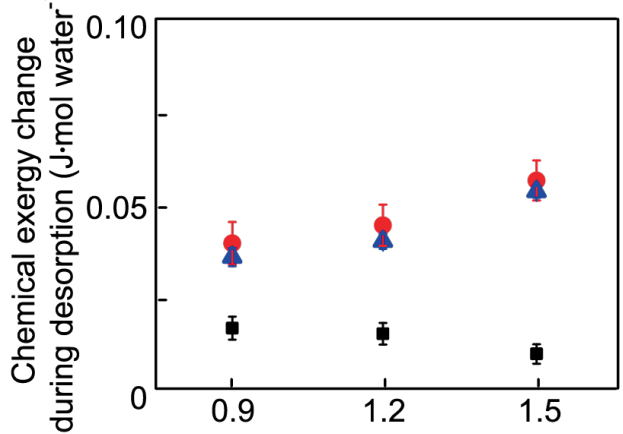

|Applied Potential during active phase| (V)

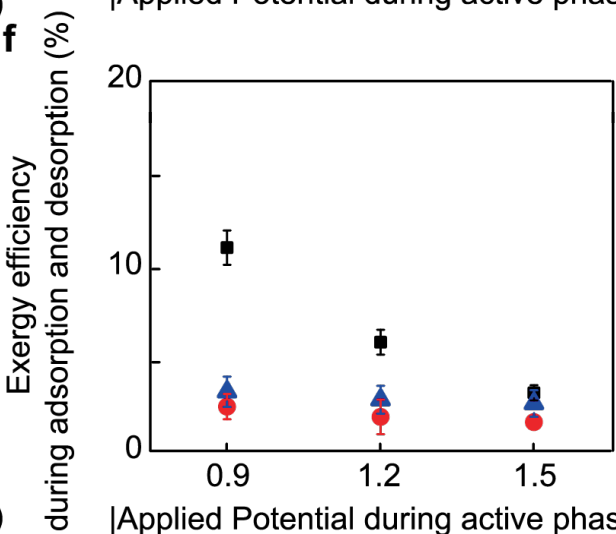

|Applied Potential during active phase| (V)

h

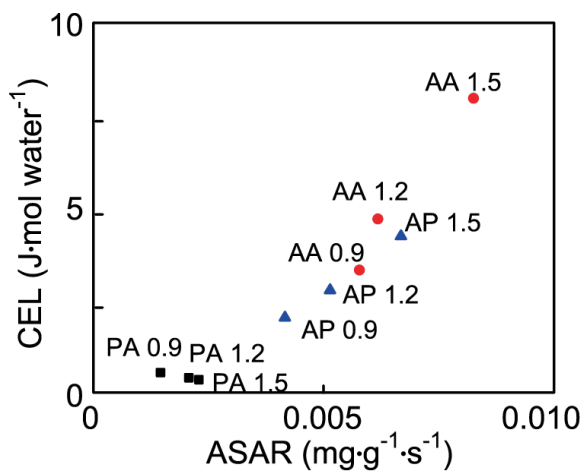

Figure S3.3 Comparison of Electrolyte solution model with a reference salt concentration of $35 \mathrm{~g} \mathrm{~L}^{-1}(\mathrm{a}-$ d) and Szargut model (e-h). a and $\mathbf{e}$ show the chemical exergy change during adsorption and $\mathbf{b}$ and $\mathbf{f}$ during desorption. $\mathbf{c}$ and $\mathbf{g}$ display the exergy efficiency of the entire process (\%) and $\mathbf{d}$ and $\mathbf{h}$ show the CEL versus ASAR. Experimental values for PA (black squares), AP (blue tringles), and AA (red circles). 



\section{CHAPTER 4}

\section{POLYELECTROLYTE-ACTIVATED CARBON COMPOSITE ELECTRODES FOR INVERTED MEMBRANE CAPACITIVE DEIONIZATION (IMCDI)}

A new way of desalination using capacitive deionization (CDI) technology is by inverting the potential profile (inverted capacitive deionization iCDI). This means ions adsorb to the electrodes at $0 \mathrm{~V}$ and desorb when biasing the electrodes to larger potential differences. Previously, this operation was achieved by preparing electrode materials with anionic and cationic surface charges. Here we show, as a novelty, that an inverted CDI operation is also possible with conventional activated carbon electrodes when used in combination with ion exchange membranes (inverted membrane capacitive deionization iMCDI). Further we show that, salt separation could

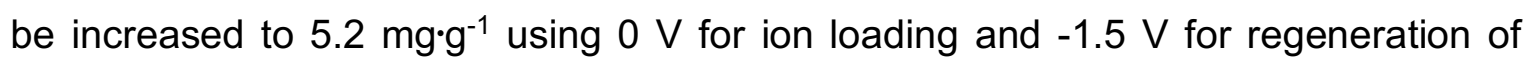
polyelectrolyte-activated carbon composite electrodes. These are made with a water soluble styrene butadiene rubber binder and positively (poly(diallyldimethylammoniumchloride)) and negatively charged (polystyrene sulfonate) polyelectrolytes and used in combination with ion exchange membranes. This leads to increased separation performance, and exergy efficiency, whereas cumulative exergy loss values remain low, indicating promising resource use efficiencies, competitive with conventional membrane capacitive deionization (MCDI).

This chapter has been published as:

P. A. Fritz, R. M. Boom, and K. Schroën. Polyelectrolyte-activated carbon composite electrodes for inverted membrane capacitive deionization (iMCDI). Sep. Purif. Technol. 220, 145-151 (2019). 


\subsection{Introduction}

Throughout the last decade capacitive deionization (CDI) has attracted great attention in water desalination (Suss et al., 2015; Tang et al., 2019). Instead of separating the water from salt as done in reversed osmosis and distillation, CDI targets the removal of the dissolved species by storing them in an electric double layer at the electrodeelectrolyte interface by applying a potential. This interface can be regenerated by neutralizing or reversing the applied potential (Oren, 2008; Porada et al., 2013; Suss et al., 2015). The incorporation of ion exchange membranes (Biesheuvel and van der Wal, 2010; Zhao et al., 2012a), or composite polymer electrodes (Gaikwad and Balomajumder, 2016), may further improve the separation performance of CDI. The charges in the membranes selectively allow permeation of one charged species, and similarly, the polymer only allows the counter ion to enter the electrode, thus charge inefficiencies originating from co-ion repulsion can be reduced.

The capacity can be further enhanced through the use of additional charges on the surface of the active electrode material, and inside the nanosized pores, which increases the amount of stored ions and reduces oxidation of the cathode (enhanced CDI) (Cohen et al., 2011; Gao et al., 2016a; Wu et al., 2015, 2016b). In contrast to active adsorption as used in conventional CDI; in inverted CDI modified electrodes with additional chemical surface charges (anode: net negative; cathode: net positive) are used to passively store ions during adsorption. This means that adsorption occurs at $0 \mathrm{~V}$ whereas desorption occurs by depolarizing the electrodes electrochemically. While this saves energy during the loading phase especially when the solution has higher conductivity, it also allows for selective adsorption of specific ions, depending on the charged groups present on the electrode surfaces.

Examples of iCDI from literature comprise carbon xerogels and pristine carbon modified with tetraethyl orthosilicate and/or nitric acid (Gao et al., 2015a, 2015b) to increase the net negative charge, and with ethylenediamine (Gao et al., 2015a) for additional net positive charge. Further, activated carbon electrodes were treated with cetrimonium bromide and sodium dodecyl benzene sulphonate and applied for the selective removal of nitrate (Oyarzun et al., 2018). A theoretical overview of CDI systems and the influence of chemical surface charges on the desalination behavior is given by Biesheuvel et al. (2015). 
In earlier work, we investigated ion exchange membranes in combination with activated carbon electrodes. An exergetic comparison revealed a higher resource use efficiency of the inverted membrane CDI (iMCDI) process compared to conventional membrane CDI (MCDI) (Fritz et al., 2018). Here we demonstrate how the efficiency of this system can be further improved by additional polyelectrolytes in the matrix of the electrodes. We investigated both $\mathrm{iMCDI}$ and $\mathrm{iCDI}$ processes, and compare classic desalination performance indicators such as salt removal capacity, and state of the art exergy indicators.

\subsection{Materials and Methods}

\subsubsection{Electrode fabrication}

Activated carbon (AC) electrodes were fabricated as described earlier (Fritz et al., 2018). For electrodes containing polyelectrolytes, activated carbon was mixed with $5 \%$ styrene butadiene rubber (Zeon, Japan) and $5 \%$ or $7.5 \%$ polymer (polystyrene sulfonate (P-), Sigma Aldrich, USA) or poly(diallyldimethyl-ammoniumchloride) $\left(\mathrm{P}_{+}\right)$, Sigma Aldrich, USA) in water (El-Deen et al., 2016b). The mixture was stirred for $13 \mathrm{~h}$ on a magnetic stirrer. Later, the AC-polyelectrolyte slurry was cast onto graphite foil with a road coater (400 $\mu \mathrm{m}$ gap) and dried over night at room temperature. Once the water evaporates the particles in the styrene butadiene rubber merge and form a cohesive network serving as binder. The electrodes were cut into circles of 1 and $5 \mathrm{~cm}$ diameter for EC-analysis and iCDI experiments respectively. The amount of active material was on average $0.135 \mathrm{~g}$ on an electrode with $5 \mathrm{~cm}$ diameter. The electrodes are termed after the percentage of polymer, e.g. for $5 \% \mathrm{P}$ - the abbreviation P-5 is used, a stack of two electrodes containing $5 \% \mathrm{P}$ - and $5 \% \mathrm{P}+$ is referred to as $\mathrm{P} 5$.

\subsubsection{Electrode characterization}

\subsubsection{Structure and element analysis}

The porous structure of the $A C$ and $P$ electrodes was imaged using scanning electron microscopy (SEM) at $2 \mathrm{kV}$ and 5000x; the pore size distribution was measured using $\mathrm{N}_{2}$ adsorption and evaluated using the BET isotherm. The chemical composition of the $A C$ and $P$ electrodes was determined using $X$-ray photoelectron spectroscopy (XPS) before and after exposure to water for $12 \mathrm{~h}$. 


\subsubsection{Electrochemical analysis}

The point of zero charge of the $A C$ and $P$ electrodes was determined using electric impedance spectroscopy with graphite foil as counter electrode and a $\mathrm{Ag} / \mathrm{AgCl}$ double junction reference electrode (Metrohm). The potential was varied between 0.3 and $-0.3 \mathrm{~V}$ at $5 \mathrm{mHz}$ with a potential deviation of $0.1 \mathrm{~V}$. The capacitance $\mathrm{C}(\mathrm{F})$ was calculated using Equation 4.1, where $Z$ "' $(\mathrm{Ohm})$ is the imaginary part of the impedance

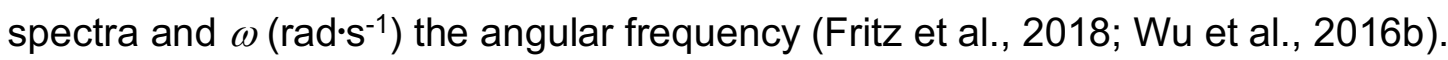

$$
C=\left|\frac{1}{\omega Z^{\prime \prime}}\right|
$$

\subsection{3. i(M)CDI experiments}

For iCDI experiments without membranes, a stack of either two AC electrodes or of one $\mathrm{P}$ - and one $\mathrm{P}+$ electrode was inserted in the CDI cell. The electrodes were prepared with the same concentration of polymer, so either both at 5 or at $7.5 \%$. A nylon mesh (diameter: $6 \mathrm{~cm}$, thickness: $125 \mu \mathrm{m}$, mesh opening: $246 \mu \mathrm{m}$, Meshtec NBC, Japan) served as spacer between the electrodes. For iMCDI experiments with membranes an anion exchange membrane (AX, molecular weight cut-of 180, AMX Neospeta, Eurodia, France) was placed in front of the $\mathrm{P}+$ electrode and a cation exchange membrane (CX, molecular weight cut-of 60, CMX Neospeta, Eurodia, France) was placed in front of the $\mathrm{P}$ - electrode. Subsequently a $\mathrm{NaCl}$ solution $\left(0.3 \mathrm{~g}^{\cdot-1}\right)$ was flushed through the cell at a flow rate of $1 \mathrm{~mL} \cdot \mathrm{min}^{-1}$ until a stable conductivity signal in the outlet was reached. Then for a period of $30 \mathrm{~min}, 0 \mathrm{~V}$ was applied (passive phase) followed by 30 min during which a potential of $-0.9,-1.2$ or $-1.5 \mathrm{~V}$ was applied (active phase). Thus, the $\mathrm{P}+$ electrode is the cathode during the active phase (negatively biased), whereas the P- electrode is the anode (positively biased). Each combination was repeated 10 times. In addition, we conducted iCDI experiments with a shorter active phase of $50 \mathrm{~s}$.

\subsubsection{MCDI experiments}

MCDI experiments where conducted with two AC electrodes and ion exchange membranes as described for iCDI experiments. While flowing $\mathrm{NaCl}$ solution $\left(0.3 \mathrm{~g} \cdot \mathrm{L}^{-1}\right)$ 
at $1 \mathrm{~mL} \cdot \mathrm{min}^{-1}$ a potential of $0.9,1.2$ or $1.5 \mathrm{~V}$ was applied for 30 min to adsorb ions followed by either $0 \mathrm{~V},-0.9,-1.2$ or $-1.5 \mathrm{~V}$ for $30 \mathrm{~min}$ to regenerate the electrodes.

\subsubsection{Indicators}

The separation performance was analyzed using established indicators such as the gravimetric salt adsorption capacity gSAC $\left(\mathrm{mg}^{\circ} \mathrm{g}^{-1}\right.$, Equation 4.2), the gravimetric salt desorption capacity gSDC ( $\mathrm{mg}^{\circ} \mathrm{g}^{-1}$, Equation 4.3), average salt absorption rate ASAR $\left(\mathrm{mg} \cdot \mathrm{g}^{-1} \cdot \mathrm{s}^{-1}\right.$, Equation 4.4), charge efficiency $\Lambda(\%$, Equation 4.5) and salt removal efficiency $\eta$ (-, Equation 4.6):

$$
\begin{gathered}
g S A C=\frac{m_{\text {salt adsorbed }}}{m_{\text {electrode }}} \\
g S D C=\frac{m_{\text {salt desorbed }}}{m_{\text {electrode }}} \\
A S A R=\frac{g S A C}{t_{c y c l e}} \\
\Lambda=\frac{m_{\text {salt adsorbed }} F}{M_{w} \int I d t} 100 \% \\
\eta=\left(1-\left(\frac{c_{a v}}{c_{0}}\right)\right)
\end{gathered}
$$

In which, $m_{\text {salt adsorbed }}$ and $m_{\text {salt desorbed }}(\mathrm{mg})$ represent the amount of salt adsorbed to or desorbed from the electrodes, respectively, $m_{\text {electrode }}(\mathrm{g})$ the active material on the electrodes, $t_{\text {cycle }}(\mathrm{s})$ the total cycle time, $M_{w}\left(\mathrm{~g} \cdot \mathrm{mol}^{-1}\right)$ the molecular weight of $\mathrm{NaCl}$, $F$ the faraday constant $\left(96485 \mathrm{C} \cdot \mathrm{mol}^{-1}\right), I\left(\mathrm{C} \cdot \mathrm{s}^{-1}\right)$ the electronic charge, $\mathrm{C}_{a v}\left(\mathrm{mg}^{-1} \mathrm{~L}^{-1}\right)$ the average salt concentration of the effluent and $c_{0}\left(\mathrm{mg}^{\circ} \mathrm{L}^{-1}\right)$ the initial salt concentration.

Furthermore the exergy efficiency $\eta_{e x}(\%$, Equation 4.7) and cumulative exergy losses CEL (J, Equation 4.8) based on the amount of purified water $\mathrm{CEL}_{n}\left(\mathrm{~J} \cdot \mathrm{mol}^{-1}\right.$, Equation 4.9) and the salt removal efficiency $\mathrm{CEL}_{\eta}\left(\mathrm{J} \cdot \mathrm{g}^{-1} \cdot \mathrm{s}^{-1}\right.$, Equation 4.10) were used to determine the resource usage of the different processes (Fritz et al., 2018). 


$$
\begin{gathered}
\eta_{\text {ex }}=\left(\frac{B_{\text {ch,out }}-B_{\text {ch, in }}}{B_{\text {el }}}\right) 100 \% \\
C E L=\sum B_{\text {in }}-\sum B_{\text {out }}=\sum B_{\text {destroyed }}+\sum B_{\text {wasted }} \\
C E L_{\text {purified water }}=\frac{C E L}{n_{w}} \\
C E L_{\text {salt removal efficiency }}=\frac{C E L}{\eta m_{\text {Electrode }} t_{\text {cycle }}}
\end{gathered}
$$

In which, $B_{c h, o u t}-B_{c h, i n}(J)$ is the difference in chemical exergy between adsorption and desorption, $B_{e l}(\mathrm{~J})$ the electrical exergy, $B_{i n}(\mathrm{~J})$ the total exergy input during adsorption and desorption, $B_{\text {out }}(\mathrm{J})$ the total exergy output during adsorption and desorption, $B_{\text {destroyed }}(\mathrm{J})$ the exergy destroyed during adsorption and desorption, $B_{\text {wasted }}$ exergy $(J)$ wasted during adsorption and desorption, and $n_{w}$ the amount of purified water (mol).

\subsection{Results and Discussion}

The styrene butadiene rubber used in this research allows fast and easy incorporation of polyelectrolytes into activated carbon electrodes without the use of toxic or environmentally harmful solvents such as DMAc. The presence of the anionic ( $\left.P_{-}\right)$and cationic $(\mathrm{P}+)$ polyelectrolytes in the electrodes and their stability after exposure to water was confirmed using SEM (Figure 4.1 and S4.1) and XPS analysis (Figure 4.2). The smooth surface in the SEM image taken of the P-5 electrode (Figure $4.1 \mathrm{~b}$ ) is representative for all polymer treated electrodes, and indicative of the presence of binder and polyelectrolytes in the porous structure of the activated carbon that is much rougher as can be seen in Figure $4.1 \mathrm{a}$.

The chemical composition measured with XPS (Figure 4.2) indicates the presence of a N1s peak in the P+ electrodes, and an S2p peak in the electrodes modified with $\mathrm{P}$-, before and after exposure to water, which is indicative of the presence of the polymer in the electrodes. Also the presence of $\mathrm{Cl}$ and $\mathrm{Na}$ ions in the respective electrodes indicates the presence of the according counter ion on the amino and sulfonate groups. The $\mathrm{C} / \mathrm{O}$ ratios that are 10.8 for $\mathrm{AC}, 8.3$ for $\mathrm{P}$-, and 14.2 for $\mathrm{P}+$ 
electrodes respectively, (Table S4.1) show that for $\mathrm{P}$ - electrodes the ratio is lower due to oxygen in the sulfonate and for $\mathrm{P}+$ electrodes it is higher due to the high proportion of carbon molecules within the polymer.

a

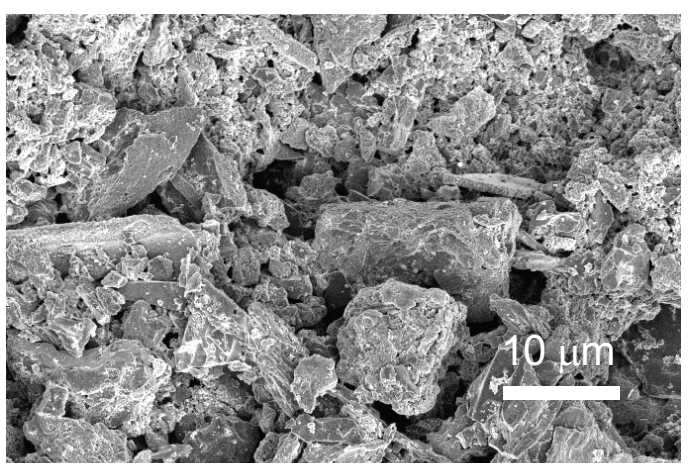

Figure 4.1. SEM images of a AC b P-5 electrodes.

a

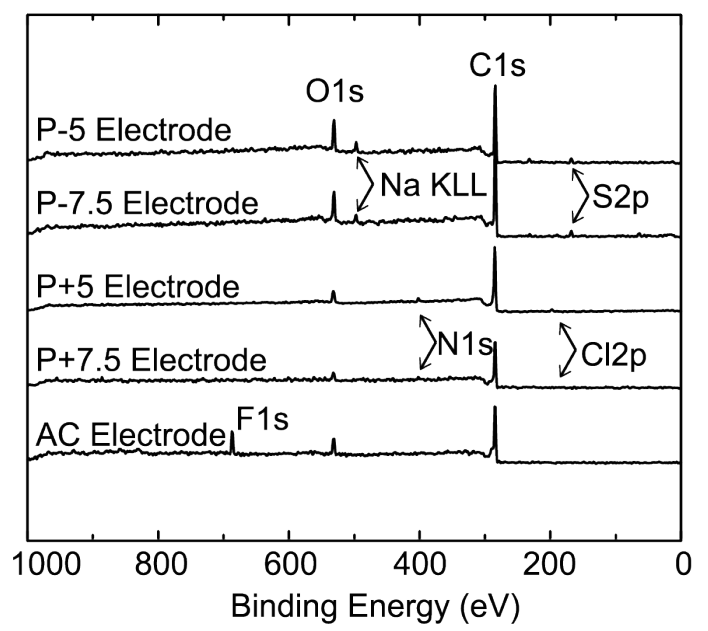

b

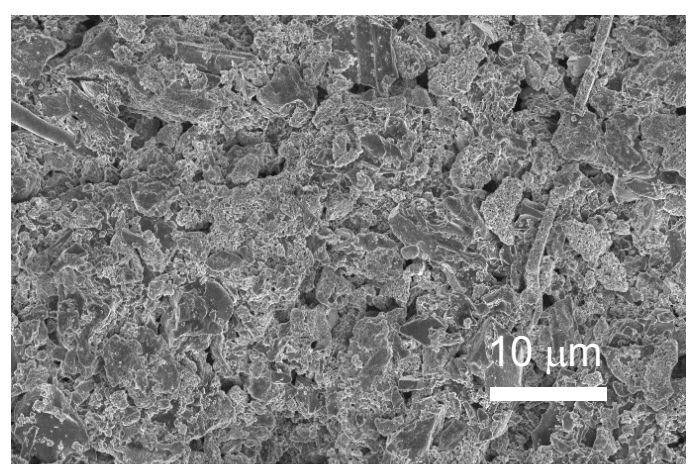

b

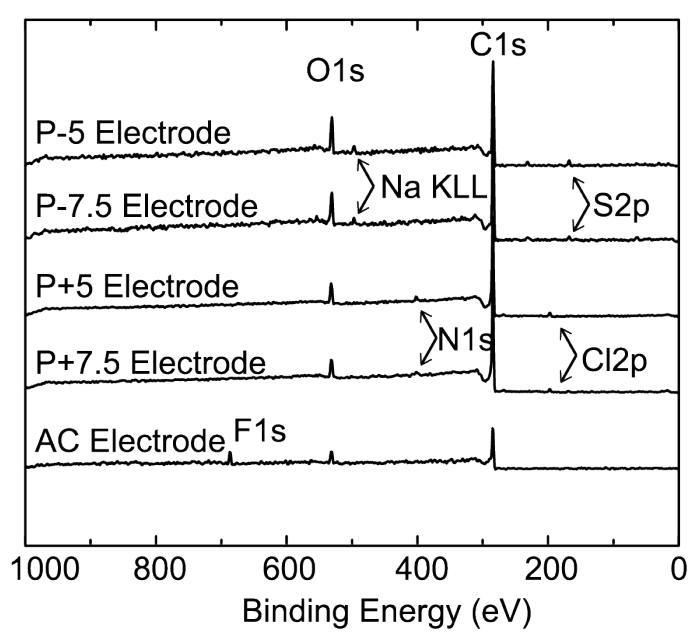

Figure 4.2. XPS spectra of the different electrodes $\mathbf{a}$ before and $\mathbf{b}$ after exposure to water.

Of the pore volume of the original activated carbon, approximately $60 \%$ can be preserved after binding with PVDF or styrene butadiene rubber to the current collector. The majority of the pores are either micropores in the range of 8 to $14 \AA$ or mesopores between 20 and $100 \AA$ in all materials (Figure 4.3).

Due to the presence of the polymers the specific capacitance increases and the point of zero charge (PZC) shifts to higher and lower values in the case of anionic and cationic charges, respectively (Figure 4.4, Figure S4.4). The effect can be enhanced by using ion exchange membranes in front of the electrodes, as indicated by $A X$ and 
CX in Figure 4.4. This implies that the bias between two electrodes at open circuit conditions can be increased by using electrode pairs with oppositely charged polyelectrolytes, using $\mathrm{AC}$ electrodes in combination with ion exchange membranes, or using both options simultaneously which renders the greatest effect.

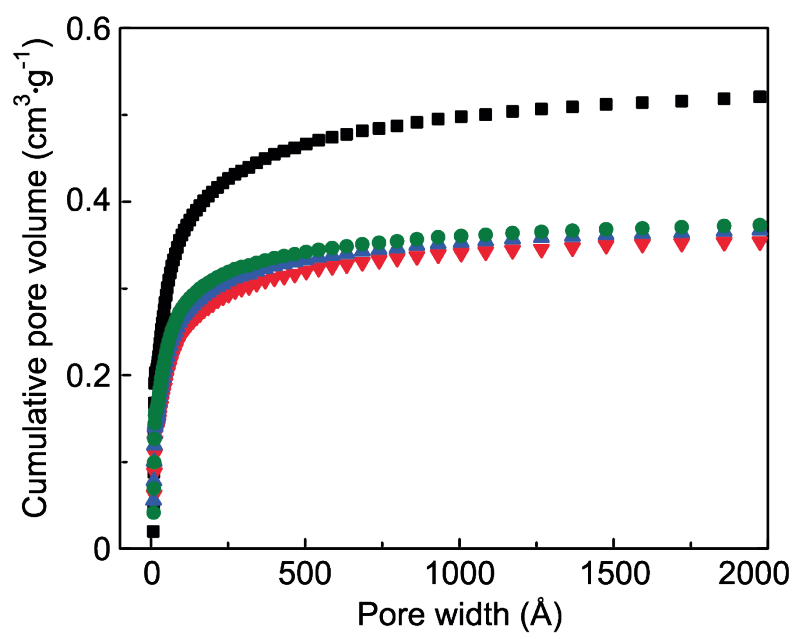

Figure 4.3. Cumulative pore volume measured by BET adsorption $\left(\mathrm{N}_{2}\right)$ of untreated $\mathrm{AC}$ material (black squares), AC (red downward pointing triangles), P+7.5 (blue upward pointing triangles) and P-7.5 (green circle) electrodes.

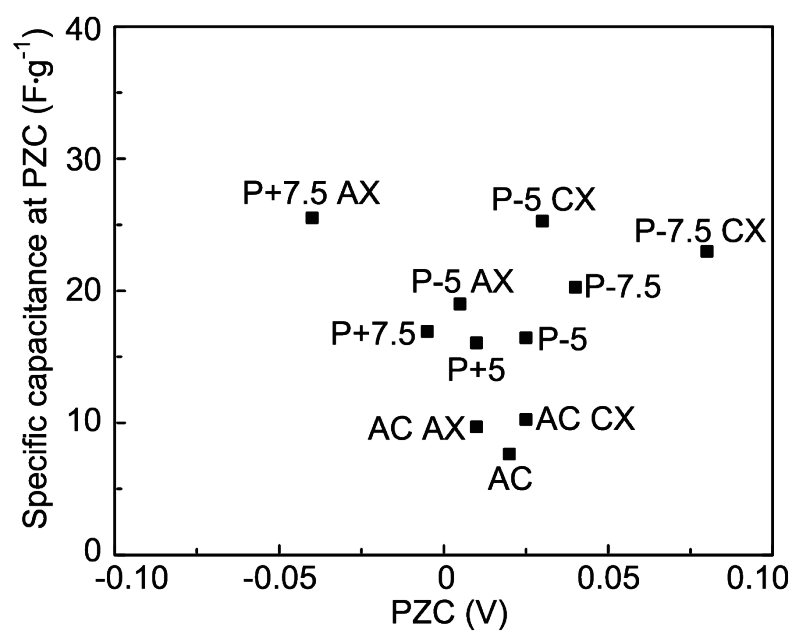

Figure 4.4. Point of zero charge of an $A C$ electrode as well as composite electrodes with cationic $(P+)$ and anionic (P-) polyelectrolyte with and without anion (AX) and cation (CX) exchange membrane. The numbers indicate the amount of polyelectrolyte present in the electrodes. (Capacitance spectra are depicted in Figure S4.4).

As expected from Figure 4.4 the incorporation of the polyelectrolytes allows to absorb and store ions due to electrostatic interactions with the charges in the polyelectrolyte also while applying a OV difference between the electrodes. Thus, a negative peak occurs during the passive phase when either 5 (P5, blue line, 
Figure 4.5 a) or $7.5 \%$ electrodes (P7.5, red line, Figure 4.5 a) are used, indicating salt adsorption. As soon as a potential bias of for example $-0.9 \mathrm{~V}$ is applied to the electrodes in the active phase the conductivity is increasing, indicating desorption. If the active phase is extended to for example $1800 \mathrm{~s}$ an adsorption peak can be detected after the desorption peak in the active phase (Figure S4.2). This is because the potential applied for desorption is larger than the working voltage windows, ranging between 0.015 and $0.12 \mathrm{~V}$ depending on the electrode combination (Figure 4.4). For AC electrodes (yellow line, Figure 4.5 a) that do not contain polymers, the opposite behavior is detected, similar to a normal CDI process (ions are adsorbed when the electrodes are biased and desorbed when OV is applied).

a

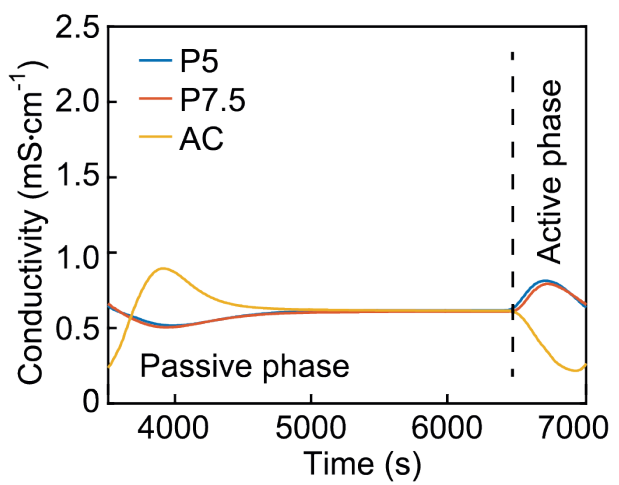

b

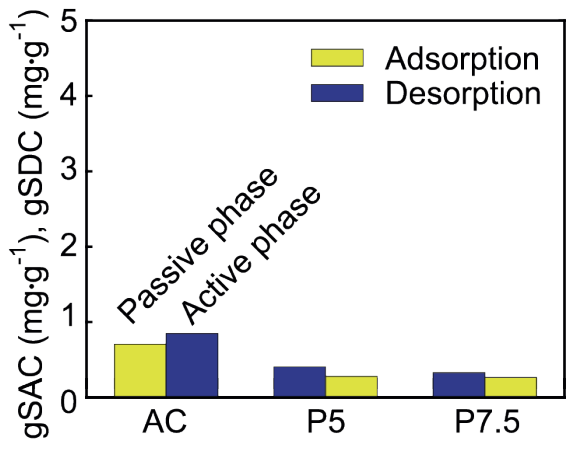

Figure 4.5. a Change in conductivity during passive $(0 \mathrm{~V})$ and active phase $(-0.9 \mathrm{~V})$ of iCDI processes without membrane: blue line P5 electrodes, red line: P7.5 electrodes, yellow line: AC electrodes. b Adsorbed (purple bars) and desorbed (yellow bars) amount of salt during passive (0 V) and active phase $(-0.9 \mathrm{~V})$ of iCDI processes.

If however ion exchange membranes are positioned in front of the electrodes it is possible to obtain an adsorption peak with simple AC electrodes when OV is applied and a desorption peak when the electrodes are biased, leading to an inversion of the conventional capacitive deionization procedure (Figure 4.6 a). With $\mathrm{P}$ electrodes gSAC values doubled in comparison to the $A C$ electrodes and quadrupled in comparison to the system without membranes (Figure 4.5 b, 4.6 b). In fact, by adding additional polyelectrolytes into the electrodes and using ion exchange membranes in the system the obtained gSAC is similar to the values obtained for a conventional MCDI process using the same AC electrodes. For example the gSAC obtained using iMCDI with P-electrodes and a regeneration at -1.2 or $-1.5 \mathrm{~V}\left(4.4\right.$ and $5.2 \mathrm{mg}^{-1} \mathrm{~g}^{-1}$, respectively) is similar or even higher to the gSAC obtained for a MCDI system using

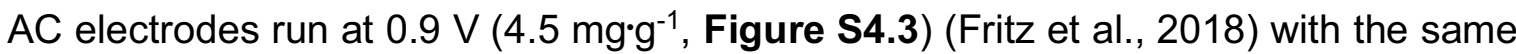


electrode material. This indicates the great potential of an inverted membrane capacitive deionization process (iMCDI) to improve the performance of iCDI systems that already have a high gSAC $\left(5 \mathrm{mg}^{-1} \mathrm{~g}^{-1}\right)$ due to their electrode material properties as described for example by Gao et al. (2015a).

a

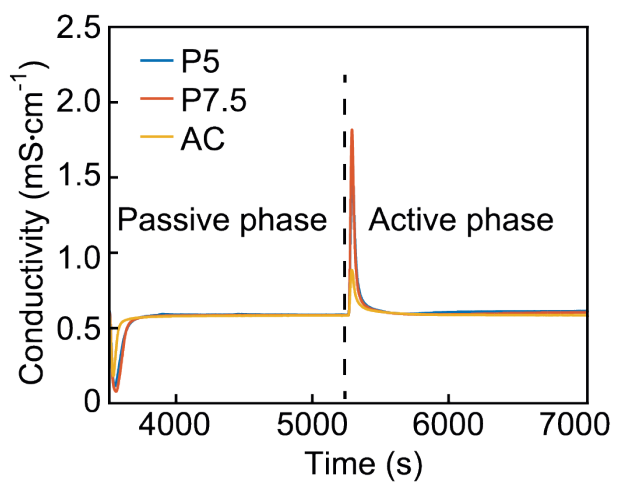

b

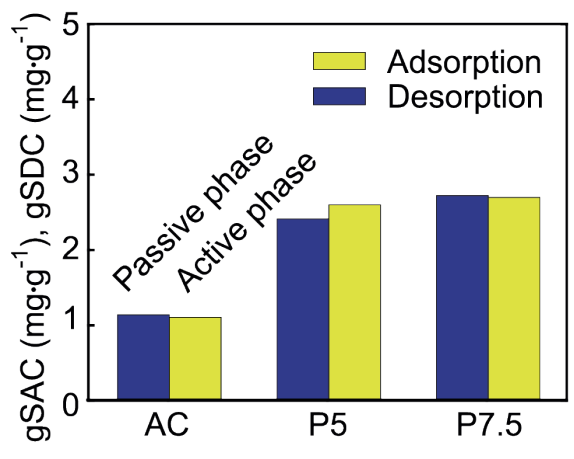

Figure 4.6. a Change in conductivity during passive and active phase $(-0.9 \mathrm{~V})$ of iCDI processes with membranes. Yellow line: AC electrodes, blue line: P5 electrodes, red line: P7.5 electrodes. b Adsorbed (purple bars) and desorbed (yellow bars) amount of salt during passive and active phase (-0.9 V) of iCDI processes with membrane.

The great difference between systems without (Figures 4.5) and with ion exchange membranes (Figures 4.6) is due to the shielding of the electrodes, thus preventing counter-ion adsorption (Schematic 4.1), as argued for MCDI (Biesheuvel and van der Wal, 2010). This allows charge efficiency values up to $85 \%, 5.5$ times higher compared to the systems without membranes (Figure 4.7) even at potentials beyond the working potential window. If no membranes are present counter-ions can access the electrode more readily, which can be especially a problem during the active phase, where the electrodes are biased with an applied potential. Figure S4.2 for example indicates that if the active phase of the system without membranes is extended from $50 \mathrm{~s}$ to $1800 \mathrm{~s}$ not only a desorption peak is observed but also a large adsorption peak, representing the ions that are electrochemically stored in the electrodes due to the applied potential (dashed arrows in Schematic 4.1). During the passive phase we thus see a large desorption peak and the adsorption peak that we can detect in the runs with shorter active cycle in Figure $\mathbf{4 . 5}$ is no longer visible. 

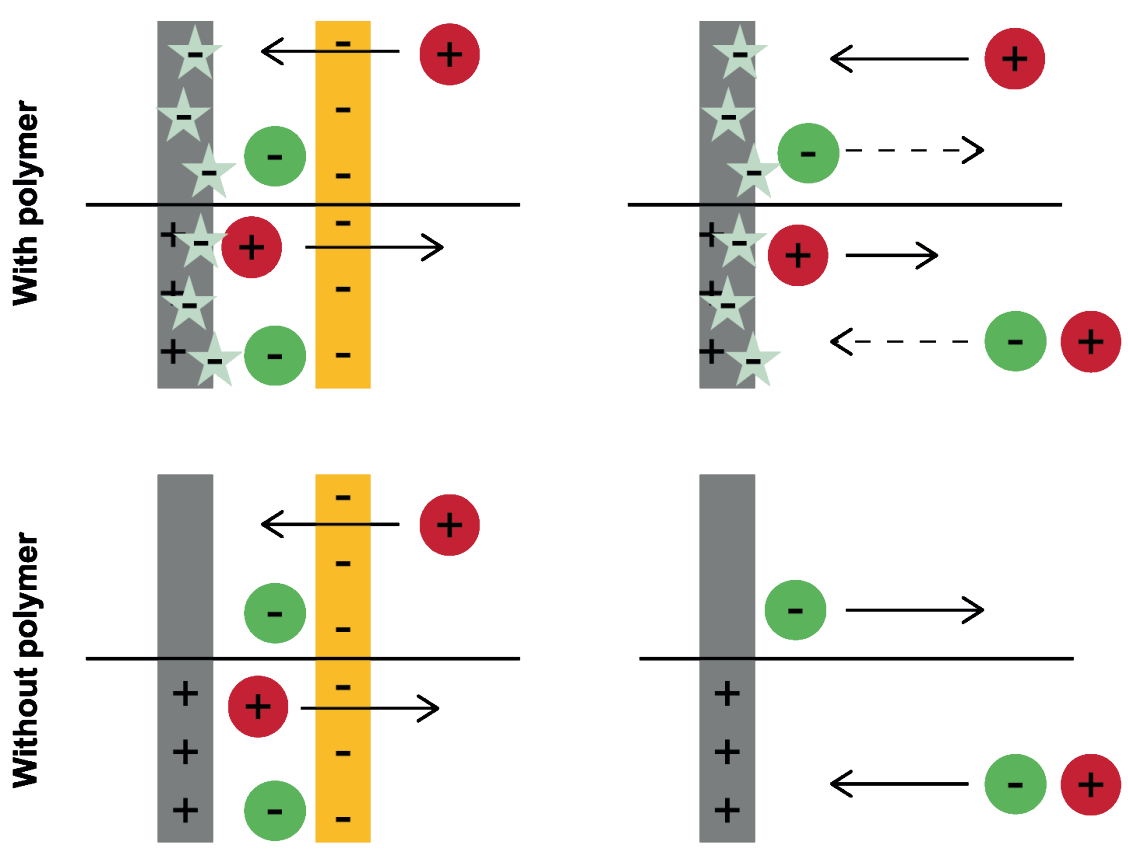

Electrode

Ion exchange membrane

Polyelectrolyte

Schematic 4.1. Ion movements in the iCDI systems, with and without membranes, and with and without polymers. Note: Only a half cell is drawn in this schematic, for a complete set up a counter electrode is required (e.g. in the scenario top right an anion exchange membrane would be added and an electrode with a positively charged polymer would be used as counter electrode).

a

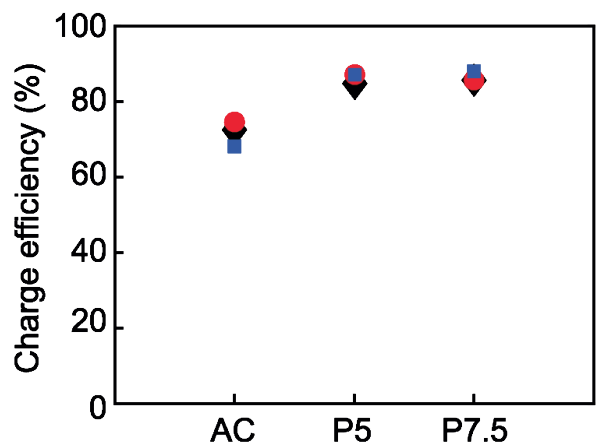

b

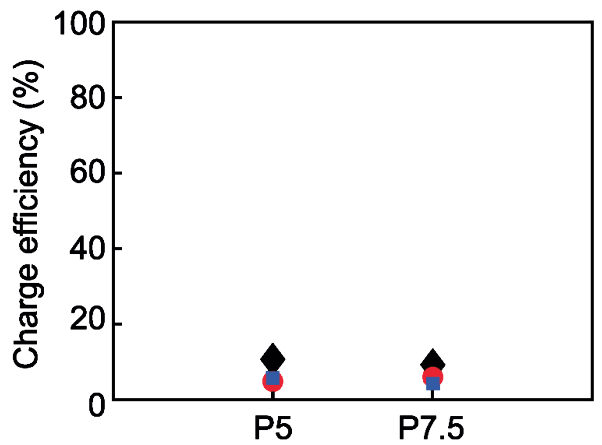

Figure 4.7. Charge efficiency for systems a with ion exchange membrane (AD cycle time: $1800 \mathrm{~s}$, DE cycle time: $1800 \mathrm{~s}$ ) and $\mathbf{b}$ without ion exchange membrane (AD cycle time: $300 \mathrm{~s}$, DE cycle time: $50 \mathrm{~s}$ ). Black diamond: $-0.9 \mathrm{~V}$, red circle: $-1.2 \mathrm{~V}$ and blue square: $-1.5 \mathrm{~V}$ applied during the active phase.

Also with respect to exergy indicators the use of membranes has a great impact. Exergy efficiency indicates how much electrical exergy needs to be invested to achieve desalination of the feed stream, and is a result of electronic and ionic resistances within the hardware and across the electrode-electrolyte interface, the membranes and the 
spacer (Dykstra et al., 2016). Without membranes, exergy efficiency is around $2 \%$ but it is increasing to $13.4 \%$ when membranes are used (Figure 4.8), which is higher than the value reported for conventional CDI (4\%) (Fritz et al., 2018).

a

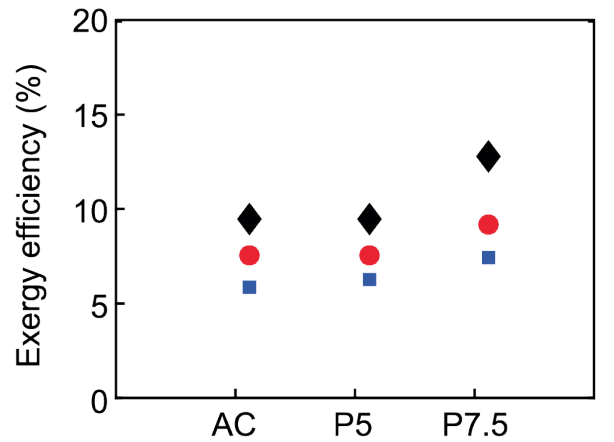

b

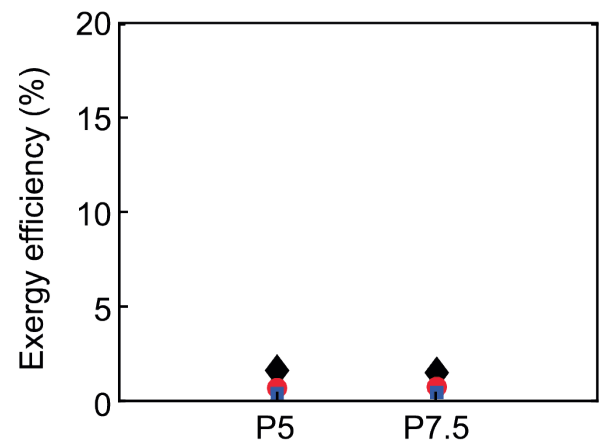

Figure 4.8. Exergy efficiency for systems a with ion exchange membrane (AD cycle time: $1800 \mathrm{~s}, \mathrm{DE}$ cycle time: $1800 \mathrm{~s}$ ) and $\mathbf{b}$ without ion exchange membrane (AD cycle time: $300 \mathrm{~s}$, DE cycle time: $50 \mathrm{~s}$ ). Black diamond: $-0.9 \mathrm{~V}$, red circle: $-1.2 \mathrm{~V}$ and blue square: $-1.5 \mathrm{~V}$ applied during the active phase.

Next to electric exergy losses also chemical exergy losses related to waste streams, play a role when considering the resource use efficiency of the process. All together are reflected as cumulative exergy losses in Figure 4.9 either related to the amount of water purified (a) or the amount of salt separated over time (b). The smallest CEL values were obtained for iMCDI systems, indicating that more salt can be separated at lower electric exergy loss and faster processing times. The addition of the polymers accentuates these values; in comparison to AC electrodes, higher ASAR values are measured at only a small increase in CEL purified water values. In fact, the ASAR values obtained for iMCDI processes using $P$ electrodes ( -1.2 and $-1.5 \mathrm{~V}$ ) have similar ASAR values as MCDI process conducted at 0.9 and $1.2 \mathrm{~V}$ (Figure 4.9 a). The trend

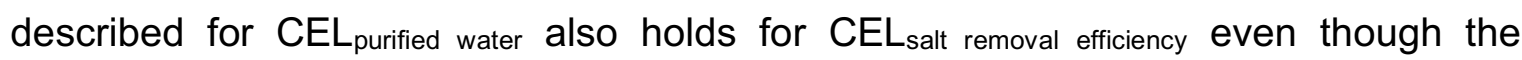
differences, especially for the processes with membranes, are smaller (Figure 4.9 b).

When comparing the cumulative exergy loss and average salt adsorption rate, it is clear that the usage of membranes (Figure $4.9 \mathrm{a}$, filled symbols vs. open symbols) and the incorporation of polymers (Figure 4.9 a, black symbols vs. red and blue symbols) are beneficial. The polymer as well as the membranes increase separation efficiency and resource use efficiency. An iMCDI process with P electrodes is similarly effective as conventional $(\mathrm{M}) \mathrm{CDI}$, and the electrode modification method described in this paper is instrumental in achieving this. It also shows the great potential to further increase the separation performance of other electrode materials that have proven to 
be beneficial for iCDI, when operated as iMCDI, also at applied potentials closer to the working potential window (Gao et al., 2015b, 2018). In addition to that iCDI and iMCDI both have the potential advantage of allowing selective removal of ions depending on the affinity of the target ion for the chemical charges in the electrodes, thus having a major advantage for separation application in more complex feed streams.
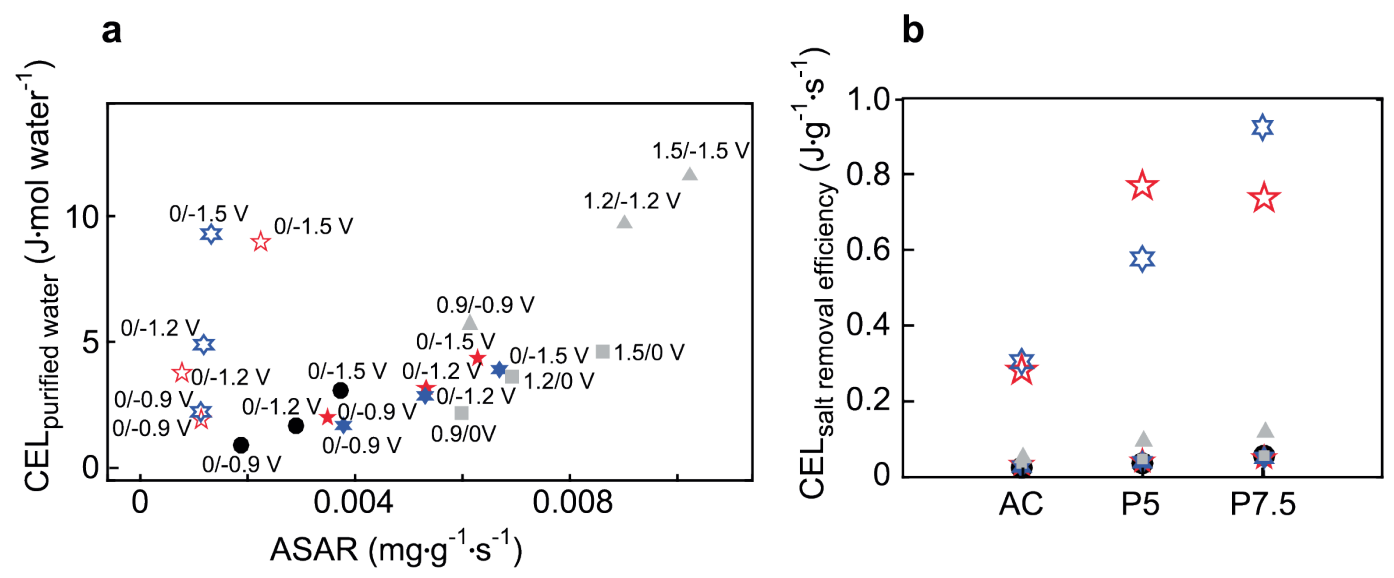

Figure 4.9. a Cumulative exergy loss based on purified water as function of average salt adsorption rate. b Cumulative exergy loss based on salt removal efficiency Black filled circle: AC electrodes with membranes (Adsorption: $0 \mathrm{~V}$, Desorption: $-0.9,-1.2$, or $-1.5 \mathrm{~V}$ see label). Red filled pentagram: P5 electrodes with membranes, Blue filled hexagram: P7.5 electrodes with membranes. The empty symbols denote corresponding systems without membranes. Gray square: AC electrodes with membranes (Adsorption: 0.9, 1.2, or $1.5 \mathrm{~V}$, Desorption $0 \mathrm{~V}$ ). Gray triangle: same process but now with desorption at $-0.9,-1.2$, or $-1.5 \mathrm{~V})$.

\subsection{Conclusion}

We showed that an iCDI process can be realized by using polyelectrolyte-activated carbon composite electrodes prepared using water soluble styrene butadiene rubber. This leads to behavior that is rather different from that reported for normal $A C$ electrodes that accumulate salt during application of an externally applied potential. The polyelectrolyte-activated carbon composite electrodes accumulate salt during a passive adsorption phase, and expel this upon applying a potential. We used these electrodes as such, and in combination with ion exchange membranes. The maximum amount of separated salt is $5.2 \mathrm{mg}^{-1}$ at a regeneration potential of $-1.5 \mathrm{~V}$ if ion exchange membranes are used. This leads to an increase in salt removal rate at a relatively low cumulative exergy loss of $1 \mathrm{~J} \cdot \mathrm{mol}^{-1}$. This makes the approach presented here competitive to conventional $\mathrm{CDI}$ and MCDI while also considering that, as extra 
feature, the addition of chemical charges or polyelectrolytes in the electrode matrix may allow selective ion adsorption.

\subsection{Supporting Information}
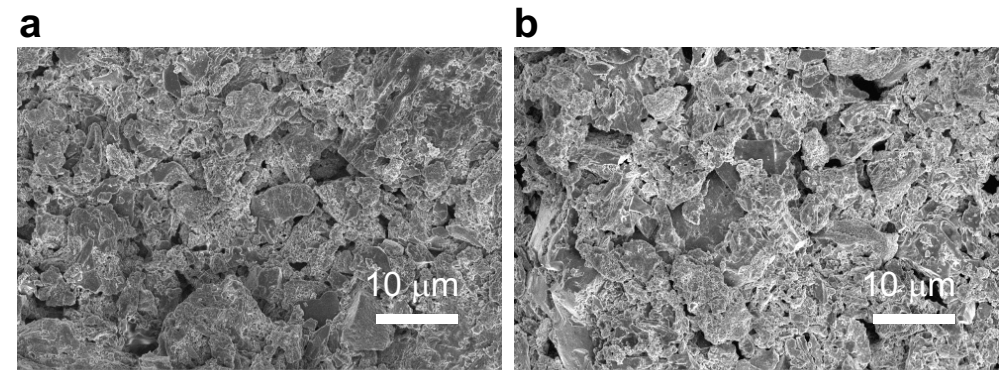

C

Figure S4.1. SEM images of a MAC-5, b PSS-7.5, c MAC-7.5

Table S4.1. Elemental composition of electrodes.

\begin{tabular}{lccccccc}
\hline \multirow{2}{*}{ Electrode } & \multicolumn{1}{c}{ At \% } \\
& $\mathbf{C}$ & $\mathbf{O}$ & $\mathbf{F}$ & $\mathbf{N a}$ & $\mathbf{S}$ & $\mathbf{C l}$ & $\mathbf{N}$ \\
\hline AC (before washing) & 85.2 & 7.7 & 7.1 & 0 & 0 & 0 & 0 \\
\hline AC (after washing) & 86.5 & 8.1 & 5.4 & 0 & 0 & 0 & 0 \\
\hline P+5 (before washing) & 91.5 & 5.4 & 0 & 0 & 0 & 1.2 & 1.9 \\
\hline P+5 (after washing) & 91.1 & 6.2 & 0 & 0 & 0 & 1.1 & 1.6 \\
\hline P+7.5 (before washing) & 89.4 & 6.9 & 0 & 0 & 0 & 1.6 & 2.1 \\
\hline P+7.5 (after washing) & 89.1 & 7.2 & 0 & 0 & 0 & 1.4 & 2.3 \\
\hline P-5 (before washing) & 81.0 & 9.0 & 0 & 7.7 & 2.3 & 0 & 0 \\
\hline P-5 (after washing) & 83.9 & 8.8 & 0 & 5.5 & 1.8 & 0 & 0 \\
\hline P-7.5 (before washing) & 76.1 & 10.8 & 0 & 10 & 3.1 & 0 & 0 \\
\hline P-7.5 (after washing) & 78.8 & 10.3 & 0 & 8.2 & 2.7 & 0 & 0 \\
\hline
\end{tabular}


a

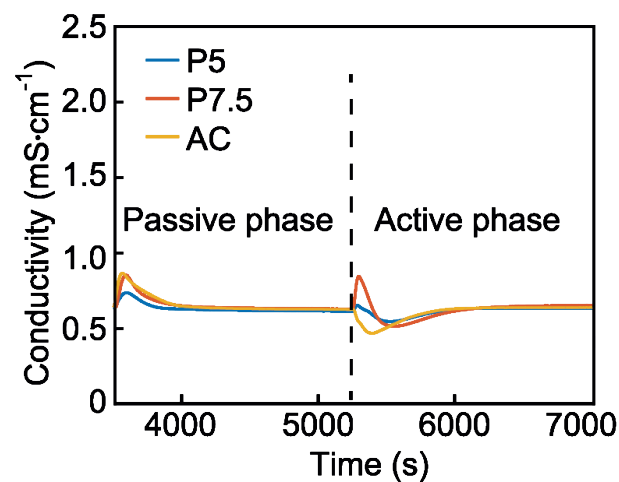

b

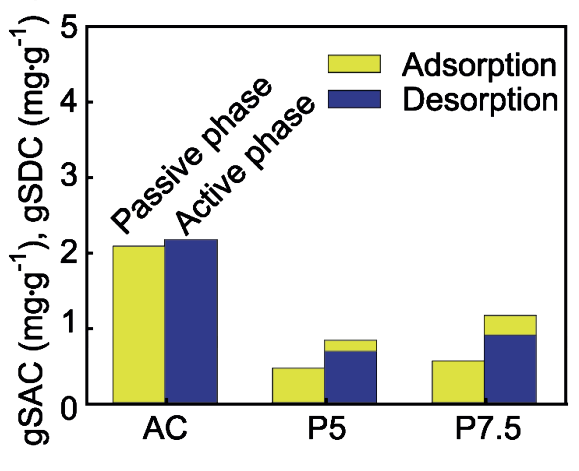

Figure S4.2. a Change in conductivity during passive and active phase $(-0.9 \mathrm{~V})$ of iCDI processes without membranes. Yellow line: AC electrodes, blue line: P5 electrodes, red line: P7.5 electrodes. b Adsorbed (purple bars) and desorbed (yellow bars) amount of salt during passive and active phase $(-0.9 \mathrm{~V})$ of iCDI processes without membrane.

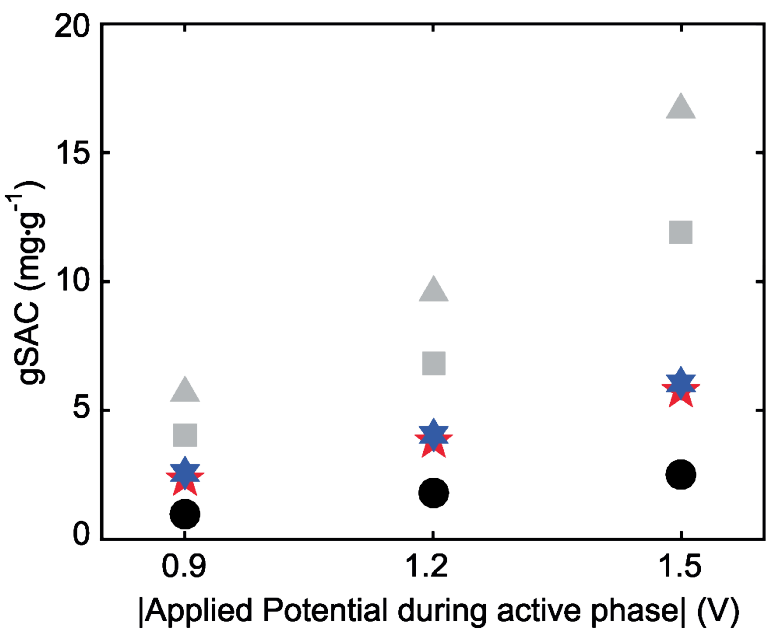

Figure S4.3. gSAC values versus the absolute electrode potential applied during the electrochemically active phase in the process for AC (black circles, large), P5 (red pentagram) and P7.5 (blue hexagram) stack using an iMCDI set up with ion exchange membranes (Adsorption: OV, desorption: -0.9,

-1.2 or $-1.5 \mathrm{~V}$ ) and $\mathrm{AC}$ electrodes with ion exchange membranes in a MCDI set up. Gray square: Adsorption $0.9,1.2$ or $1.5 \mathrm{~V}$, desorption: 0V. Gray triangle: Adsorption $0.9,1.2$ or $1.5 \mathrm{~V}$, desorption: $-0.9,-1.2$ or $-1.5 \mathrm{~V}$ 


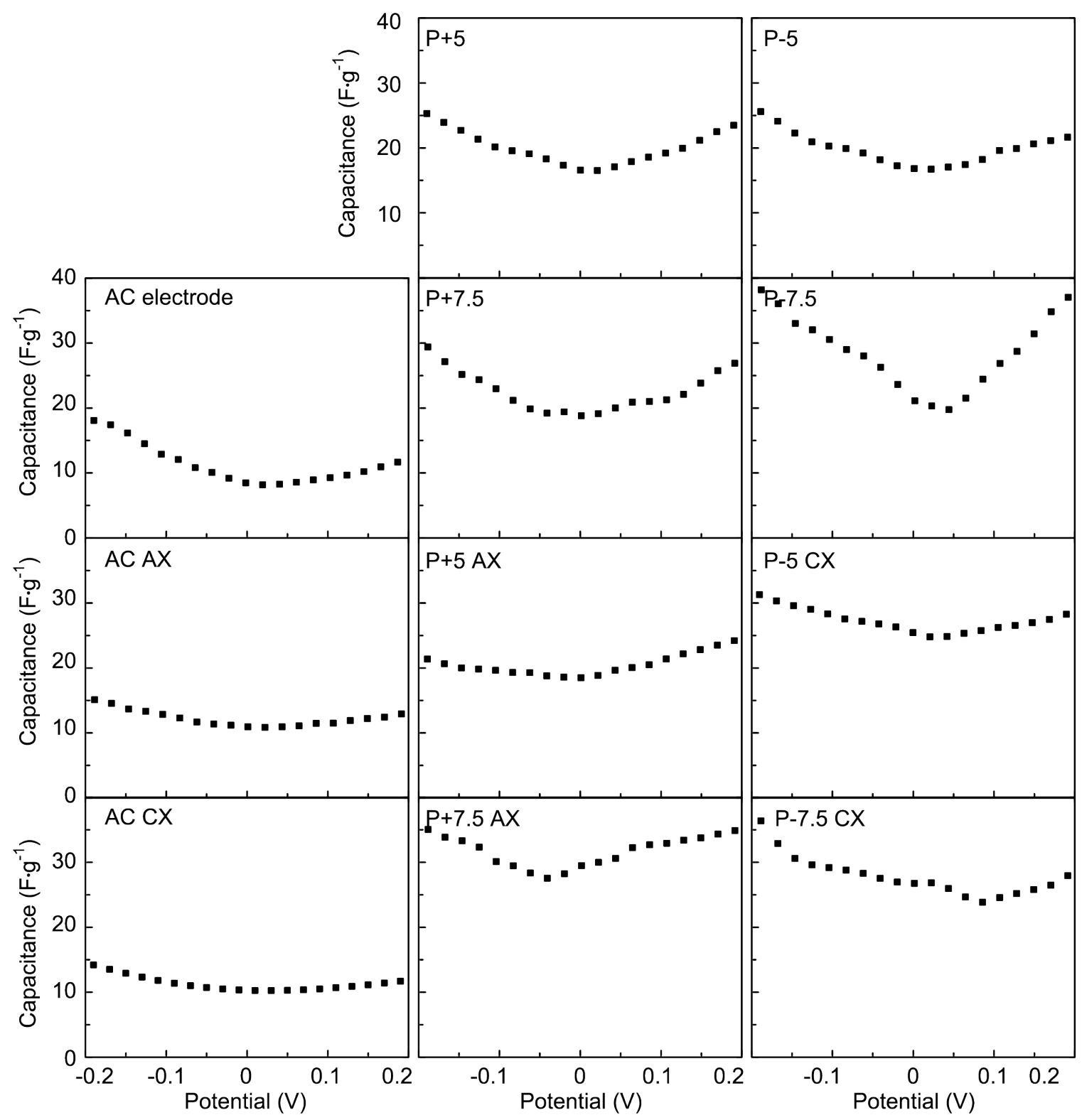

Figure S4.4. Capacitance values depending on potential applied to various electrodes as specified in the plots (minimum indicates position of PZC). 



\section{CHAPTER 5}

\section{ZWITTERIONIC POLYMER MODIFIED}

\section{POROUS CARBON FOR HIGH PERFORMANCE}

\section{AND ANTIFOULING CAPACITIVE DESALINATION}

Capacitive deionization (CDI) is an emerging technology for effective brackish water desalination to address fresh water scarcity. It is of great interest due to its high energy efficiency, environmental friendliness, and low-cost operation, compared with traditional desalination technologies. However, electrode fouling, caused by dissolved organic matter and resulting in reduction of electrode electrosorption capacity and device lifespan, is an impediment to practical application of CDI. Herein, we report a novel salty water desalination electrode with excellent antifouling properties. The antifouling electrode is prepared by coating zwitterionic polymer brushes, i.e., poly (sulfobetaine methacrylate) (SBMA), on porous carbon (PC) via surface-initiated atom transfer radical polymerization. The successful coating of zwitterionic polymer on PC surface is confirmed by TEM, SEM, XPS, TGA, and other characterizations. Coating with polySBMA did not affect the electrosorption capacity of PC electrodes and imparted antifouling properties (versus fouling by model foulant bovine serum albumin) during long-term salt removal tests (100 desalination/regeneration cycles). This is an important step toward practical application of capacitive deionization technology for brackish water desalination.

This chapter has been published as:

P. Zhang, P. A. Fritz, K. Schroën, H. Duan, R. M. Boom, and M. B. Chan-Park. Zwitterionic polymer modified porous carbon for high-performance and antifouling capacitive desalination. Appl. Mater. Interfaces 10, 33564-33573 (2018). 


\subsection{Introduction}

In recent decades it has become evident that freshwater scarcity, due to steadily increasing demand and changing climate conditions, will become a threat to sustainable global development. In its most recent annual risk report, the World Economic Forum lists water crises as the largest global risk in terms of potential impact. Around 4.0 billion people suffer from severe water scarcity for over one month of the year. Half a billion people live under the condition of severe water scarcity all year round (Mekonnen and Hoekstra, 2016; World Economic Forum, 2017). To meet the increasing demand for fresh water, various water treatment innovations and technologies have been researched and developed, including reverse osmosis, electrodialysis, distillation, and others.

However, these conventional technologies suffer from high energy consumption, for example, because of the requirement of e.g. high pressure operation (Liu et al., 2017). Also known as electrosorption, capacitive deionization (CDI) has been attracting enormous interest in the desalination field because of its low energy consumption, cost effectiveness, environmental friendly operation, and absence of secondary pollution, compared with conventional desalination technologies. The concept of $\mathrm{CDI}$ is based on the working principle of an electrochemical double-layer (EDL). When an external electrostatic field is applied between electrodes, ionic species with opposite charges are electro-adsorbed in the EDL formed at the interface between the solution and the porous electrode interface until the pores are saturated with ions. After being saturated with ions, the electrodes can be regenerated by a short circuit or a reverse voltage (Dahanayaka et al., 2017; El-Deen et al., 2016a). Within the CDI field, researchers have mainly focused on novel electrode material development, theoretical study, device architecture design, and performance improvement (Huang et al., 2017; Suss et al., 2015). Only a few studies have been done on electrode fouling, which is essential for practical application of CDI technology since electrode fouling results in diminished salt removal capacity during long-term or repetitive operation (Gao et al., 2016b).

Generally, due to their high electrical conductivity and easily tunable structure, porous carbon materials-based electrodes have been considered as ideal electrodes for CDI applications. Different kinds of carbon materials, such as activated carbon, 
graphene, carbon nanotubes, carbon aerogel and their composites, have been intensively investigated as CDI electrodes. Likewise, porous carbon (PC) materials have been widely adopted as carbon electrodes in supercapacitors, due to their low cost, excellent capacity and superior stability. These outstanding properties make PC interesting for application in CDI electrodes. However, application of pristine PC in water treatment is hindered by a) the presence of protein and/or other organics in practical applications and b) the intrinsic hydrophobicity, which together can result in electrode fouling and reduction of the electrosorption capability and device lifespan. Therefore, modification of porous carbon to have a surface that resists proteins and/or other organics is critical for practical CDI applications.

Conjugation of hydrophilic polymer brushes is the most commonly employed approach for preparing anti-fouling surfaces (Gao et al., 2017a; Rosso et al., 2011; Su et al., 2017b). Zwitterionic polymers, such as phosphobetaine, carboxybetaine, and sulfobetaine, have been observed to have excellent anti-fouling properties ( $\mathrm{Ji}$ et al., 2012; Mi et al., 2015; Wang et al., 2015a; Zhao et al., 2015). The underlying mechanism is that zwitterionic polymers, carrying both anionic and cationic groups in one molecule, can strongly bind water molecules due to electrostatically induced hydration (Chen et al., 2005; He et al., 2008).

Surface-initiated atom transfer radical polymerization (SI-ATRP) is a very useful and efficient approach to modify or functionalize different kinds of materials, such as silicon, metal oxides, gold, carbon, CNTs, graphene oxide, etc. (Song et al., 2016a). We report herein the first example of an anti-fouling zwitterionic polymer brush coated porous carbon electrode for capacitive deionization. The anti-fouling coating material, poly(sulfobetaine methacrylate), is formed on the PC electrode via SI-ATRP (Scheme 5.1); the resulting electrode material is hereafter referred to as PC-SBMA. We firstly fabricated PC via a simple one-pot "leavening method" by heating cellulose with $\mathrm{KHCO}_{3}$ at high temperature under argon atmosphere. The as-prepared PC, with good conductivity and large surface area, underwent deposition of polydopamine (PDA) in a Tris buffer. Because of strong $\pi-\pi$ interaction, a strongly adherent PDA coating is readily and uniformly produced on the surface of the carbon (Song et al., 2016b). The synthesized PC-PDA was then further modified by immobilizing 2bromoisobutyryl bromide (BiBB) initiator and, subsequently, grafting of polySBMA via SI-ATRP to grow a zwitterionic polymer layer on the PC surface. The resulting PCSBMA electrode was tested for electrosorption capacity and antifouling performance 
in a long-term test of salt removal in the presence of protein. The obtained results are relevant to water desalination and food processes.

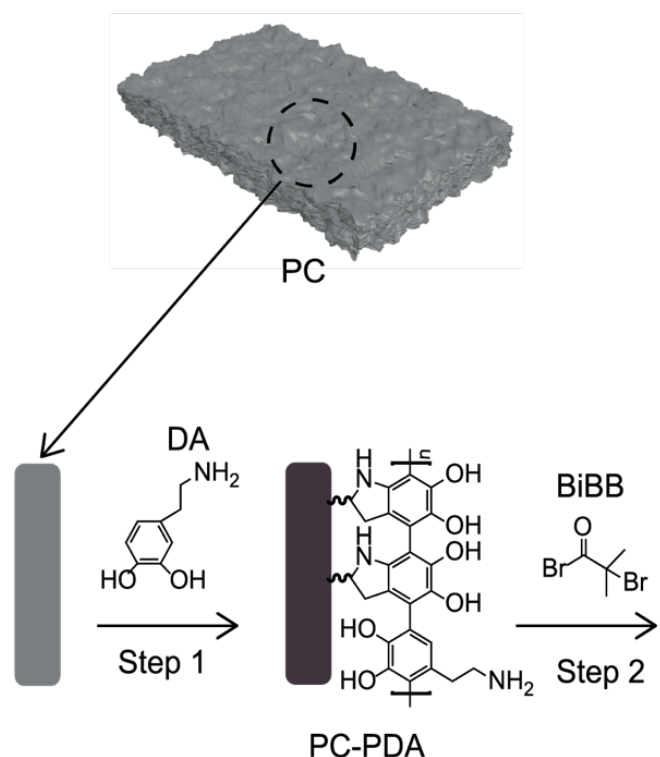

PC-PDA

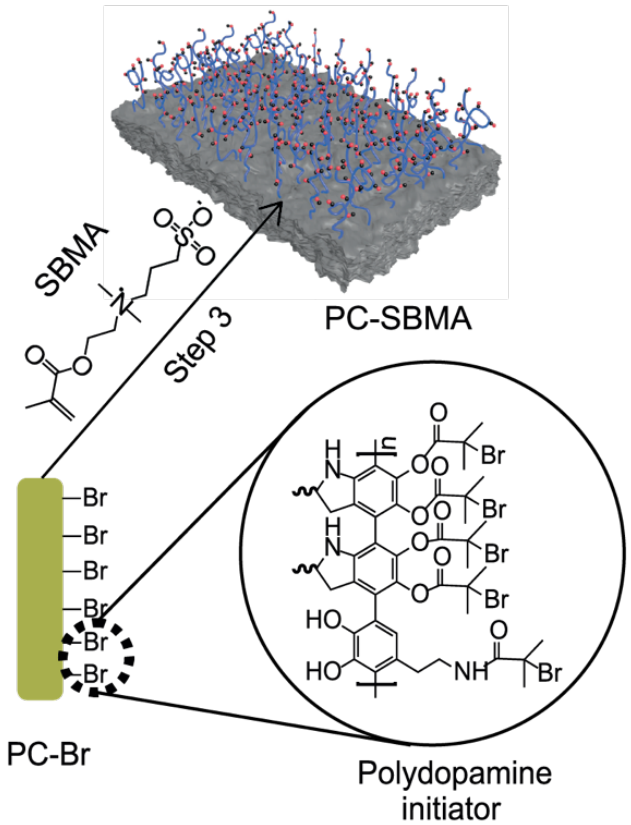

Scheme 5.1. Illustration of the porous carbon modification process.

\subsection{Materials and methods}

\subsubsection{Materials}

a-Cellulose, potassium bicarbonate (99.7 \%), hydrochloric acid (37\%), triethylamine (TEA, $99 \%$ ), dopamine hydrochloride (DA), a-bromoisobutyryl bromide (BiBB, $98 \%$ ), Trizma base, methanol (99.8\%), ethanol (99.8\%), $\mathrm{N}, \mathrm{N}, \mathrm{N}, \mathrm{N}$ ",N"-pentamethyldiethylenetriamine (PMDETA, $99 \%$ ), copper(II) bromide $\left(\mathrm{CuBr}_{2}, 99 \%\right)$, tetrahydrofuran (THF, anhydrous, $\left.99.9 \%\right)$, ethyl a-bromoisobutyrate (EBiB, $98 \%$ \%), N-(3-sulfopropyl)- $N$-(methacryloxyethyl)- $N, N$-dimethylammonium betaine (SBMA, $97 \%$ ), polyvinylidene fluoride (PVDF), anhydrous dimethylacetamide (DMAc), and bovine serum albumin (BSA, lyophilized powder) were purchased from Sigma Aldrich. Water used in all experiments was deionized and purified with a Merck Millipore system; and the deionized water had a resistivity of more than $15 \mathrm{M} \Omega \cdot \mathrm{cm}$. Copper wire with diameter of $1 \mathrm{~mm}$ was washed with hydrochloride acid and rinsed with water before use. 


\subsubsection{Characterization}

A field-emission scanning electron microscope (FESEM; JEOL-6700F) and a transmission electron microscope (TEM; JEOL, JEM-2010) equipped with an energydispersive X-ray spectrometer (EDS) were used to characterize the morphology and structure of the samples. Water contact angles were measured by a DSA25 Contact Angle Analyzer (Kruss, Germany). X-ray diffraction (XRD) measurements were collected using a wide-angle X-ray diffractometer (Bruker D2 Phaser) with Cu Ka radiation $(\lambda=0.154 \mathrm{~nm})$. Raman spectra were measured using a Raman spectrometer (Renishaw InVia Reflex) with a $514 \mathrm{~nm}$ laser as the excitation source. Thermogravimetric Analysis (Netzsch STA 409) was performed to analyze the composition and thermal behavior. Before measurements, all samples were completely dried to remove water and were subjected to TGA under nitrogen atmosphere. X-ray photoelectron spectroscopy (XPS) of the as-prepared samples was performed using an ESCALAB MK-II spectrometer (VG Scientific Ltd., West Sussex, Britain).

\subsubsection{Electrochemical measurements}

The working electrodes for electrochemical characterization were prepared as follows: carbon powders $(10 \mathrm{mg})$ in $1 \mathrm{~mL}$ ethanol were subjected to $30 \mathrm{~min}$ sonication to form a homogenous suspension. $10 \mu \mathrm{L}$ of this suspension was dropped onto a glass carbon electrode (GCD, $\varnothing=0.6 \mathrm{~mm}$ ) and dried at room temperature for 2 hours. After drying, $10 \mu \mathrm{L}$ of a Nafion solution was added onto it to make sure that the carbon materials bound to the GCD. The electrochemical tests were performed using a $\mathrm{CHI}$ 660D electrochemical workstation in a three-electrode system with a Pt foil as the counter electrode and a saturated calomel electrode (SCE) reference electrode in $10 \mathrm{mM} \mathrm{NaCl}$ deaerated aqueous solution. The specific capacitance $C$ was calculated from the relation $C=I \Delta t / m \Delta V$, where $I, \Delta t, \Delta V$, and $m$ represent the discharge current, discharge time, voltage range, and sample mass, respectively.

\subsubsection{Synthesis of PC}

$5 \mathrm{~g}$ of $\alpha$-cellulose and potassium bicarbonate at a mass ratio of 1:2 was heated at $800{ }^{\circ} \mathrm{C}$ for $90 \mathrm{~min}$ under argon atmosphere with a heating rate of $10{ }^{\circ} \mathrm{C} \mathrm{min}-1$ using a tube furnace. After heating, the sample was passively cooled to room temperature 
and then dispersed in $\mathrm{HCl}$ solution and stirred for 6 hours. PC was obtained after filtration with water and ethanol and drying at $60^{\circ} \mathrm{C}$ in vacuum oven overnight.

\subsubsection{Polydopamine (PDA) coating}

PC (500 mg) was dispersed and sonicated for $30 \mathrm{~min}$ in $200 \mathrm{~mL}$ of water and ethanol mixture ( $v: v=1: 1)$. Then $200 \mathrm{mg}$ DA and $2.0 \mathrm{mM}$ Tris buffer solution were successively added to the dispersion under magnetic stirring. The dopamine polymerization and coating was performed at $37{ }^{\circ} \mathrm{C}$ for $12 \mathrm{~h}$. Then, the PDA-coated PC (PC-PDA) was washed with ethanol several times by centrifugation. The separated product was dried in a vacuum oven at $60{ }^{\circ} \mathrm{C}$ for $24 \mathrm{~h}$.

\subsubsection{Immobilization of the ATRP initiator}

$250 \mathrm{mg}$ PC-PDA added into a solution comprised of $20 \mathrm{~mL}$ of anhydrous THF and $2 \mathrm{~mL}$ of TEA (14.4mmol) and then was sonicated for $20 \mathrm{~min}$. The mixture was purged with Ar for $10 \mathrm{~min}$. After that, $1.8 \mathrm{~mL}$ BiBB (14.4 mmol) dissolved in $20 \mathrm{~mL}$ anhydrous THF was added dropwise into the PC-PDA suspension, which was kept chilled in an ice bath. Then, the solution was removed from the ice bath and allowed to react at room temperature for $24 \mathrm{~h}$. The resulting product was washed with THF, acetone, and methanol several times and dried in a vacuum oven at $60^{\circ} \mathrm{C}$ for $24 \mathrm{~h}$. The $\mathrm{PC}$ with immobilized ATRP initiator is denoted as PC-Br.

\subsubsection{Preparation of porous carbon/polymer hybrids by SI-ATRP}

$250 \mathrm{mg} \mathrm{PC}-\mathrm{Br}$ was placed in a $20 \mathrm{~mL}$ Schlenk flask and sonicated for $20 \mathrm{~min}$ after adding $10 \mathrm{~mL}$ of water/methanol mixture ( $\mathrm{v}: \mathrm{v}=1: 1)$, followed by successive addition of $\mathrm{CuBr}_{2}(11 \mathrm{mg}, 0.05 \mathrm{mmol})$, PMDETA $(10 \mu \mathrm{L}, 0.05 \mathrm{mmol}), \operatorname{EBiB}(7 \mu \mathrm{L}$, $0.05 \mathrm{mmol})$, SBMA (1.397 g, $5 \mathrm{mmol}$ ), and a piece of copper wire with length of $\sim 2 \mathrm{~cm}$. The flask was sealed with a rubber stopper and the mixture was degassed by three freeze-thaw-pump cycles. The polymerization was conducted for a defined time at $60{ }^{\circ} \mathrm{C}$, the time depending on the target polymer brush length. SBMA grafted PC (PCSBMA) was separated by centrifugation and washed thrice with $\mathrm{H}_{2} \mathrm{O}$ and methanol. 


\subsubsection{CDI electrode preparation}

The CDI electrodes were made following our previous procedure with some modification, as shown in Figure S5.9 a. Briefly, a mixture of PC or PC-SBMA and PVDF with a mass ratio of 85:15 was blended in an appropriate amount of DMAc and vigorously stirred for 4 hours to achieve a homogeneous carbon slurry. The generated slurry was then pressed onto a graphite foil and dried at $80^{\circ} \mathrm{C}$ for 2 hours in a drying oven and then under vacuum at the same temperature overnight to remove any remaining solvent residue. After drying, the electrodes were soaked in $1 \mathrm{M} \mathrm{NaCl}$ aqueous solution to activate them. The final thickness of the PC or PC-SBMA layer on the graphite foil after drying was approximately $80 \mu \mathrm{m}$ according to the SEM characterizations (Figure S5.9 d and S5.9 e), and the mass of active material in the carbon electrode was $1.38 \mathrm{mg} \cdot \mathrm{cm}^{-2}$. The total active area of a pair of electrodes was $2 \times 5 \times 5 \mathrm{~cm}^{2}$.

\subsubsection{Electrosorptive capacity measurement}

The desalination/regeneration experiments were conducted using a flowthrough capacitor system. A pair of large-scale electrodes separated by a piece of nylon spacer were assembled into a homemade CDI prototype (Figure 5.5). The capacitive deionization behaviour characterizations of PC and PC-SBMA-24 electrodes were carried out at $1.2 \mathrm{~V}$ charging and $0 \mathrm{~V}$ discharging $(1.2 / 0 \mathrm{~V}$, charge and discharge periods were each $5 \mathrm{~min}$ ) with about $10 \mathrm{~L}$ of $245 \mathrm{mg}^{-\mathrm{L}^{-1}}$ deaerated $\mathrm{NaCl}$ solution (conductivity $500 \mu \mathrm{S} \cdot \mathrm{cm}^{-1}$ ) with a flowrate of $10 \mathrm{~mL} \cdot \mathrm{min}^{-1}$ using a peristaltic pump. The electrosorption capacity $\left(\mathrm{Q}, \mathrm{mg}^{-1} \mathrm{~g}^{-1}\right)$ can be calculated by multiplying the volumetric flow rate $(\Phi)$ by the time $(t)$ integral of the concentration (c) (Gao et al., 2017b):

$$
Q=\frac{\Phi}{m} \int\left(c_{t}-c_{0}\right) d t
$$

where $m$ is the mass of the activated carbon material in the electrode, and $c_{t}$ and $c_{0}$ are the salt concentrations in effluent (time dependent) and influent, respectively. 


\subsection{Results and discussion}

The porous structure and morphology of PC and PC-SBMA was characterized with field emission scanning electron microscopy (FESEM) and transmission electron microscopy (TEM). FESEM images (Figure 5.1 a, b and $\mathbf{5 . 1} \mathbf{d}, \mathbf{e}$ ) show that both PC and PC-SBMA have an open macroporous structure, with pore sizes ranging from hundreds of nanometers to several micrometers, forming a 3D hierarchically porous carbon. It is worth noting that the surface of PC seems smoother than that of modified PC-SBMA, which is indicative of the formation of a polymer layer.
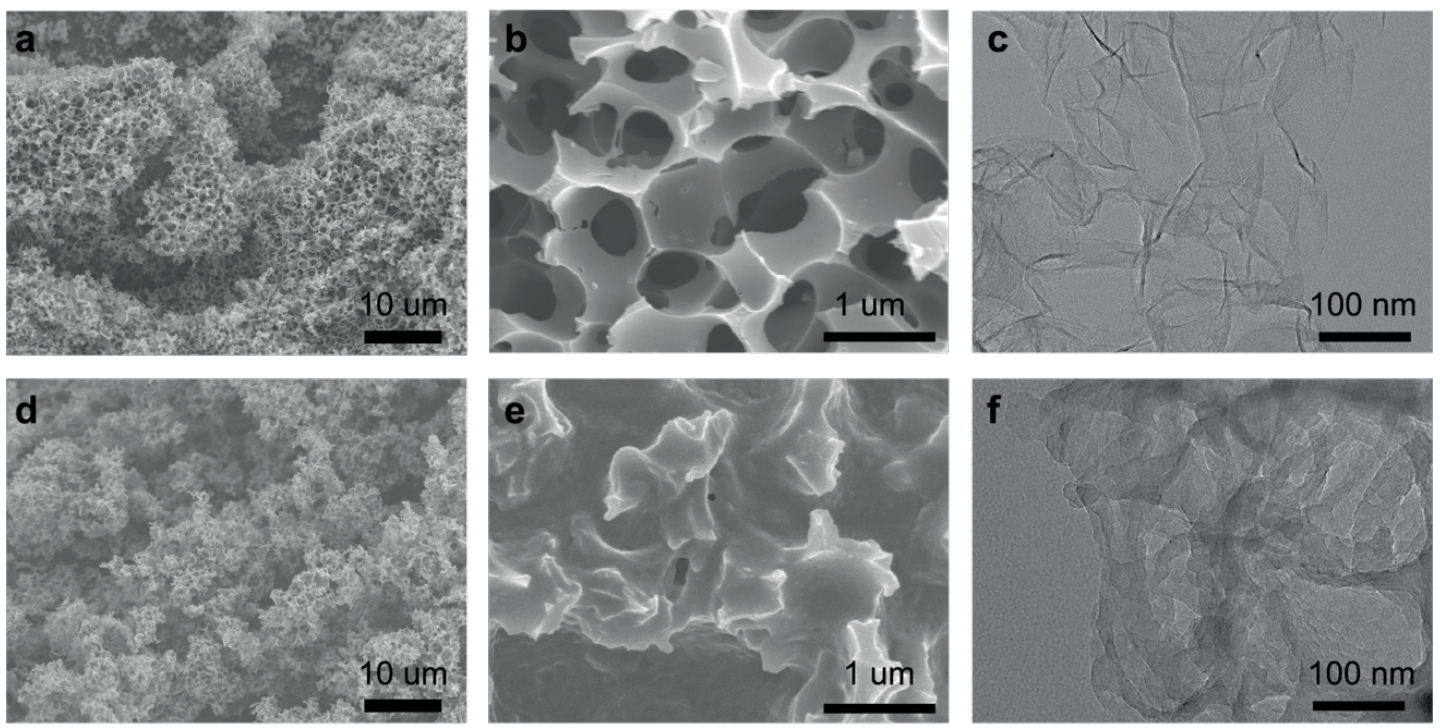

Figure 5.1. FESEM images for (a) PC, (d) PC-SBMA-24; high magnification FESEM of (b) PC, (e) PCSBMA-24; TEM images (c) PC, (f) PC-SBMA-24.

The FESEM images (Figure S5.2) of the surfaces of both PC-PDA and PC-Br also appear rougher than that of PC. The TEM images (Figure $5.1 \mathrm{c}$ and $\mathrm{f}$ ) are consistent with the macropores observed in the FESEM images. In addition, the HRTEM image (Figure S5.1 a) shows that PC contains multilayer graphene sheets with sizes of tens of nanometers, and the line profile (Figure S5.1 b) indicates that the $\mathrm{d}$-spacing of the graphene sheets is in the range of $0.34 \sim 0.39 \mathrm{~nm}$; the ring-like SAED pattern confirms that the porous carbon has a graphitic and polycrystalline structure with numerous edge defects (Bi et al., 2016a). The element distribution of PC-SBMA, measured with energy-dispersive X-ray spectroscopy (EDX)-STEM mapping 
(Figure S5.3), shows that sulfur and bromide are homogeneously distributed in the carbon surface, indicating a uniform coating of polySBMA brushes on PC surface.

The water contact angles of PC, PC-PDA, and PC-Br were $135^{\circ}, 104^{\circ}$, and $145^{\circ}$, respectively. The contact angle of PC-SBAM-24 could not be determined (Figure S5.4 d) as the water droplet was completely absorbed into the electrode within $1 \mathrm{~s}$, which is indicative of a super-hydrophilic surface obtained after coating with polySBMA brushes. The dispersibility in deionized water of PC without and with zwitterionic polymer modification was tested by standing at high concentration $\left(2.0 \mathrm{mg} \cdot \mathrm{mL}^{-1}\right)$ for three days; it is apparent (Figure S5.4 e) that the PC without modification precipitated almost entirely, while polySBMA-coated PC dispersed uniformly and stably without obvious aggregation. The much improved dispersion of PC-SBMA is attributable to its hydrophilic surface and electrostatic repulsion between PC-SBMA particles.

Porous carbon samples without and with modification were analyzed with X-ray photoelectron spectroscopy (XPS) to confirm the grafting of the polySBMA polymer brush onto the PC surfaces. From the survey spectra (Figure 5.2 a), the appearance of new peaks at 398.4 eV in PC-PDA and $68.8 \mathrm{eV}$ in $\mathrm{PC}-\mathrm{Br}$, attributed respectively to $\mathrm{N} 1 \mathrm{~s}$ and $\mathrm{Br} 3 \mathrm{~d}$, indicate the successful deposition of PDA and immobilization of BiBB on the carbon surface (Ezzat and Huang, 2016). The nitrogen (N) and sulfur (S) peaks are normally used for characterizing zwitterionic sulfobetaine on a surface (Chang et al., 2011; Chou et al., 2017). The characteristic S2p peak at $167.5 \mathrm{eV}$, due to sulfur in the zwitterionic sulfobetaine side chains, was detected in the survey scan of PC-SBMA but not in that of PC-Br (Figure $5.2 \mathrm{~d}$ and $\mathbf{S 5 . 5} \mathbf{b}$ ). In the high-resolution spectra (Figure 5.2 b, S5.5 a, and S5.5 c), N1s peaks are well-resolved into two component peaks at binding energies 401.2 and $398.4 \mathrm{eV}$, which correspond to quaternary ammonium $\left[-\mathrm{N}\left(\mathrm{CH}_{3}\right)_{2}-\right]^{+}$in the SBMA segment and $\mathrm{C}-\mathrm{N}$ in the PDA segment, respectively (Chang et al., 2011; Chou et al., 2017). Longer reaction time in the SIATRP step led to higher intensities of the N1s and S2p peaks (Figure S5.5 c and S5.5 d), which is reflected by the elemental compositions calculated from the XPS data (Table 5.1). Sulfur is present at $0.59,0.98$, and 2.20 atomic \% for PC-SBMA-6, PCSBMA-12, and PC-SBMA-24, respectively. SI-ATRP provides precise control over the molecular weight of the polymer brushes attached to the carbon surface, (Song et al., 2016a) which is reflected in the higher intensities observed. 

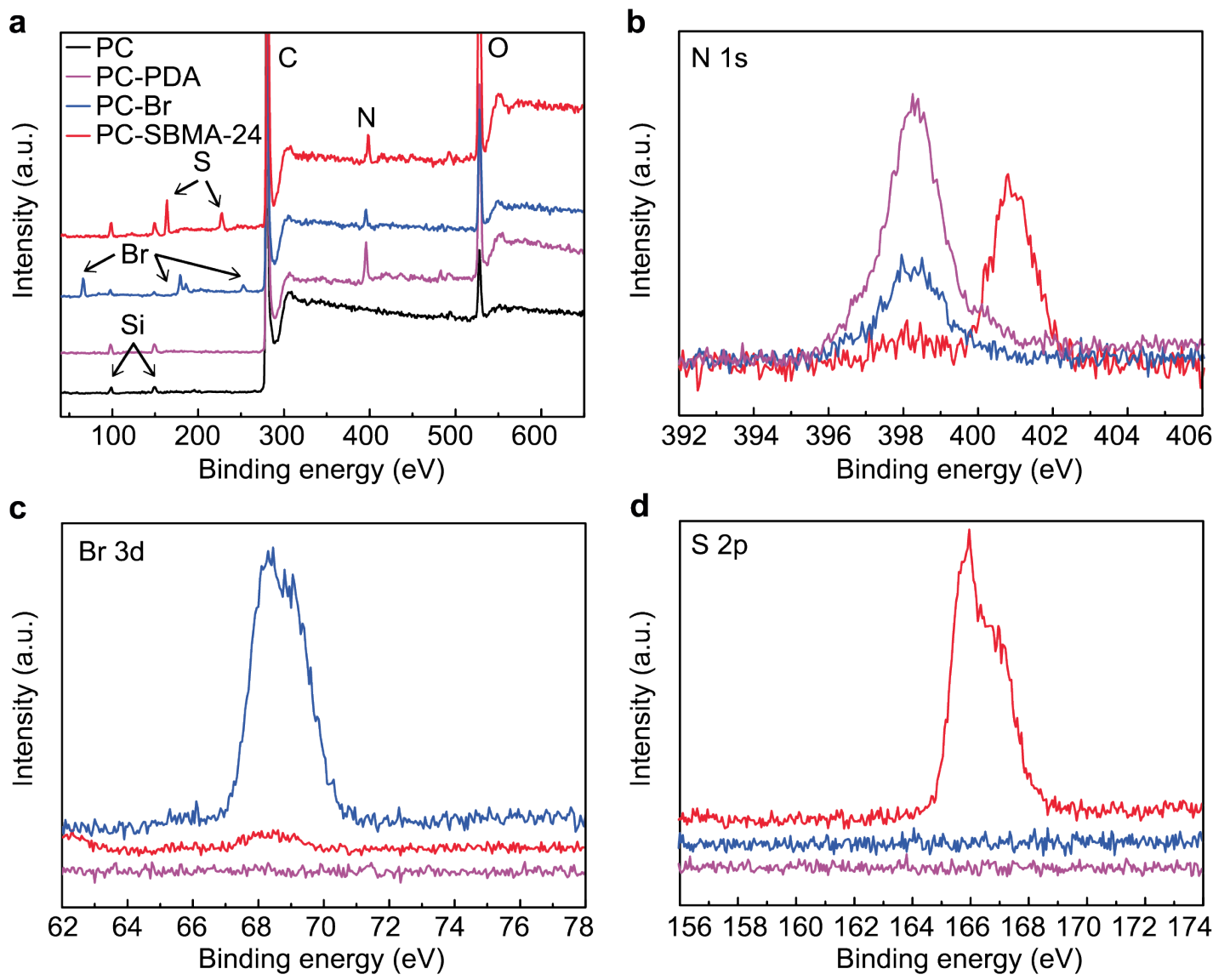

Figure 5.2. XPS spectra of porous carbon surface before and after zwitterionic polymer modification. (a) Survey spectra; high resolution spectra of PC-PDA, PC-Br and PC-SBMA-24 showing (b) N1s, (c) Br3d and (d) S2p.

Table 5.1: Surface elemental composition (\% atomic concentration) calculated from XPS for porous carbon surface before and after modification.

\begin{tabular}{lccccc}
\hline & $\mathbf{C}$ & $\mathbf{O}$ & $\mathbf{N}$ & $\mathbf{B r}$ & $\mathbf{S}$ \\
\hline PC & 96.03 & 3.97 & & & \\
PC-PDA & 80.47 & 14.41 & 5.12 & & \\
PC-Br & 86.64 & 9.18 & 2.28 & 1.12 & 0.59 \\
PC-SBMA-6 & 88.8 & 7.37 & 2.3 & 0.09 & 0.98 \\
PC-SBMA-12 & 85.93 & 9.4 & 2.21 & 0.09 & 2.20 \\
PC-SBMA-24 & 74.81 & 16.78 & 3.86 & 0.21 & \\
\hline
\end{tabular}

The Raman spectra of PC and PC-SBMA-24 (Figure 5.3 a) show two prominent peaks, the $D$ and $G$ bands at $1581 \mathrm{~cm}^{-1}$ and $1349 \mathrm{~cm}^{-1}$, respectively. It is universally acknowledged that the $\mathrm{G}$ band is due to in-plane vibration of the $\mathrm{sp}^{2}$ carbon atoms while the $D$ band is a defect-induced Raman feature representing the non-perfect crystalline structure of the material (Deng et al., 2015; Liang et al., 2014). The ratio of the intensities $\left(I_{G} / I_{D}\right)$ is a measure of the degree of structural disorder (Balogun et al., 
2016; Bi et al., 2016b; Zhu et al., 2017). Compared with pristine PC, the G/D ratio calculated for PC-SBMA-24 decreased considerably from 1.08 to 0.88 , indicating that the carbon surface defects increased with the grafting of PSBMA zwitterionic polymer brushes through SI-ATRP modification (Chen et al., 2015; Liu et al., 2010; Takada et al., 2016). The $I_{G} / I_{D}$ of PC-PDA and PC-Br also decreased to 0.97 and 0.92 (Figure S5.6), respectively, which may be caused by the successful coating with PDA and immobilization of the ATRP initiator.

a

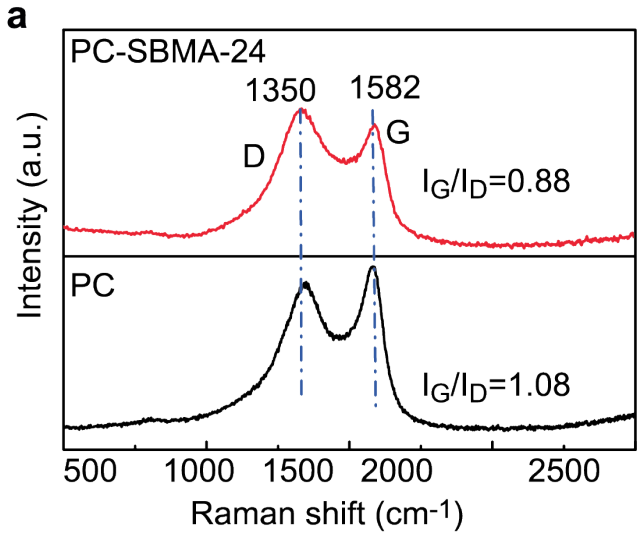

C

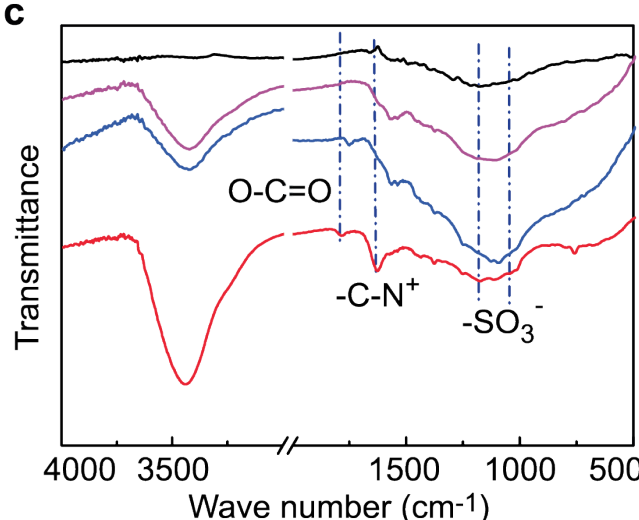

b

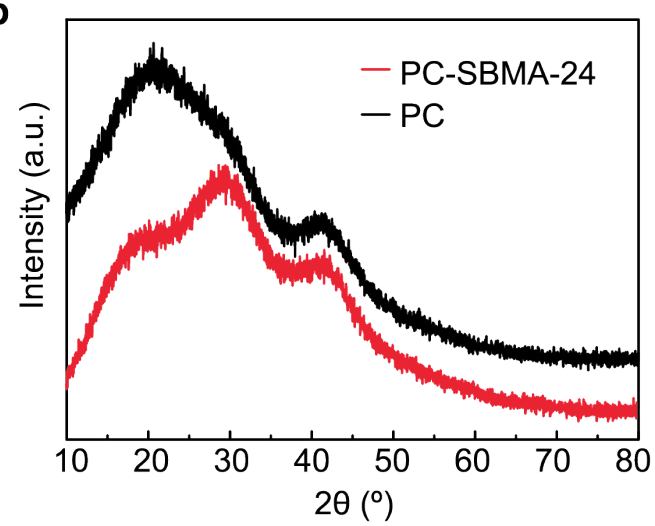

d

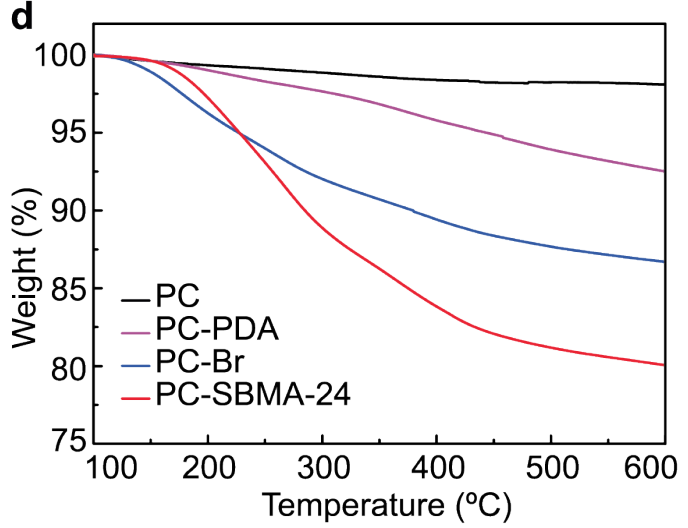

Figure 5.3. Raman spectra (a) and XRD pattern (b) for PC and PC-SBMA-24; FTIR spectra (c) and TGA curves (d) of PC, PC-PDA, PC-Br, and PC-SBMA-24.

The XRD patterns of PC electrodes before and after modification, shown in Figure $5.3 \mathrm{~b}$ and $\mathbf{S 5 . 6} \mathrm{b}$, exhibit similar diffraction features with two broad peaks centered at $2 \theta=20.0^{\circ}$ and $41.8^{\circ}$, which can be attributed to the (002) and (100) reflections, respectively (Woo et al., 2008). This characterization is perfectly consistent with the SEAD pattern in Figure S5.1 a, indicating that bare PC is a typical graphitic carbon material with some degree of graphitization (Zheng et al., 2014). The lower intensity of the (002) peak in the XRD pattern of PC-SBMA-24 implies a lower degree 
of graphitization than in PC; this may be due to additional defects introduced to the graphitic structure of PC by the modification.

In the FTIR spectrum (Figure $5.3 \mathrm{c}$ ), the presence of the polySBMA coating is indicated by the bands at $1046 \mathrm{~cm}^{-1}$ and $1180 \mathrm{~cm}^{-1}$, that are attributable to $-\mathrm{S}=\mathrm{O}$ stretch, and the band at $1791 \mathrm{~cm}^{-1}$, attributable to O-C=O stretch (Salleh et al., 2014; Zhai et al., 2014). In addition, the polySBMA spectrum also shows the bands of $-\mathrm{C}-\mathrm{N}^{+}$stretch at $1636 \mathrm{~cm}^{-1}$ (Zhai et al., 2014); these results confirm the successful coating of the polySBMA brush to the carbon surface.

Thermogravimetric analysis (TGA) is a powerful technique to determine the composition and thermal stability of materials. Figure $\mathbf{5 . 3} \mathrm{d}$ shows that the ultimate mass loss for PC, PC-PDA, PC-Br, and PC-SBMA-24 was $1.8 \%, 7.4 \%, 13.2 \%$, and $19.9 \%$, respectively. The mass loss derivative peaks (Figure S5.7 a) for PC-Br and PC-SBMA appeared at ca. $175^{\circ} \mathrm{C} \& 260{ }^{\circ} \mathrm{C}$, and $270{ }^{\circ} \mathrm{C} \& 390{ }^{\circ} \mathrm{C}$, respectively. The variations in thermal degradation patterns among the different forms of PC confirm the presence of different functionalities on the PC (which by itself has a featureless derivative TGA curve) (Hatton et al., 2017). The mass loss increases with the extension of ATRP reaction time (Figure $\mathbf{S 5 . 7}$ b), indicating that control of reaction time controls the amount of zwitterionic polymer that is grafted on the carbon surface.

Various electrochemical measurements were conducted to investigate the performance of PC and PC-SBMA-24 as CDI electrode. First, cyclic voltammetry (CV) experiments on the prepared material in $1 \mathrm{M} \mathrm{NaCl}$ aqueous deaerated solution were conducted with a potential window from -0.4 to $0.6 \mathrm{~V}$, to evaluate the electrochemical properties for CDI (Figure 5.4 a, S5.8 a and S5.8 b). No redox peak was observed in CV curves for both PC and PC-SBMA-24, suggesting that they can function as electrodes in typical electrical double layer capacitors (EDLCs) based on coulombic interactions rather than electrochemical redox reactions (Aslan et al., 2016; Liu et al., 2017; Wang et al., 2016, 2015b). In addition, the CV curves exhibit a nearly perfect rectangular shape, which means the charge current can reach the plateau quickly when applying a reverse voltage, suggesting that ionic species can be rapidly and effectively adsorbed to and desorbed from the electrode. The CV curves remain approximately rectangular even at $100 \mathrm{mV} \cdot \mathrm{s}^{-1}$ (Figure $\mathbf{S 5 . 8}$ a and $\mathbf{S 5 . 8} \mathbf{~ b}$ ), implying a rapid movement of ions into and out of PC and PC-SBMA-24 electrode surface even at high scan rate. 

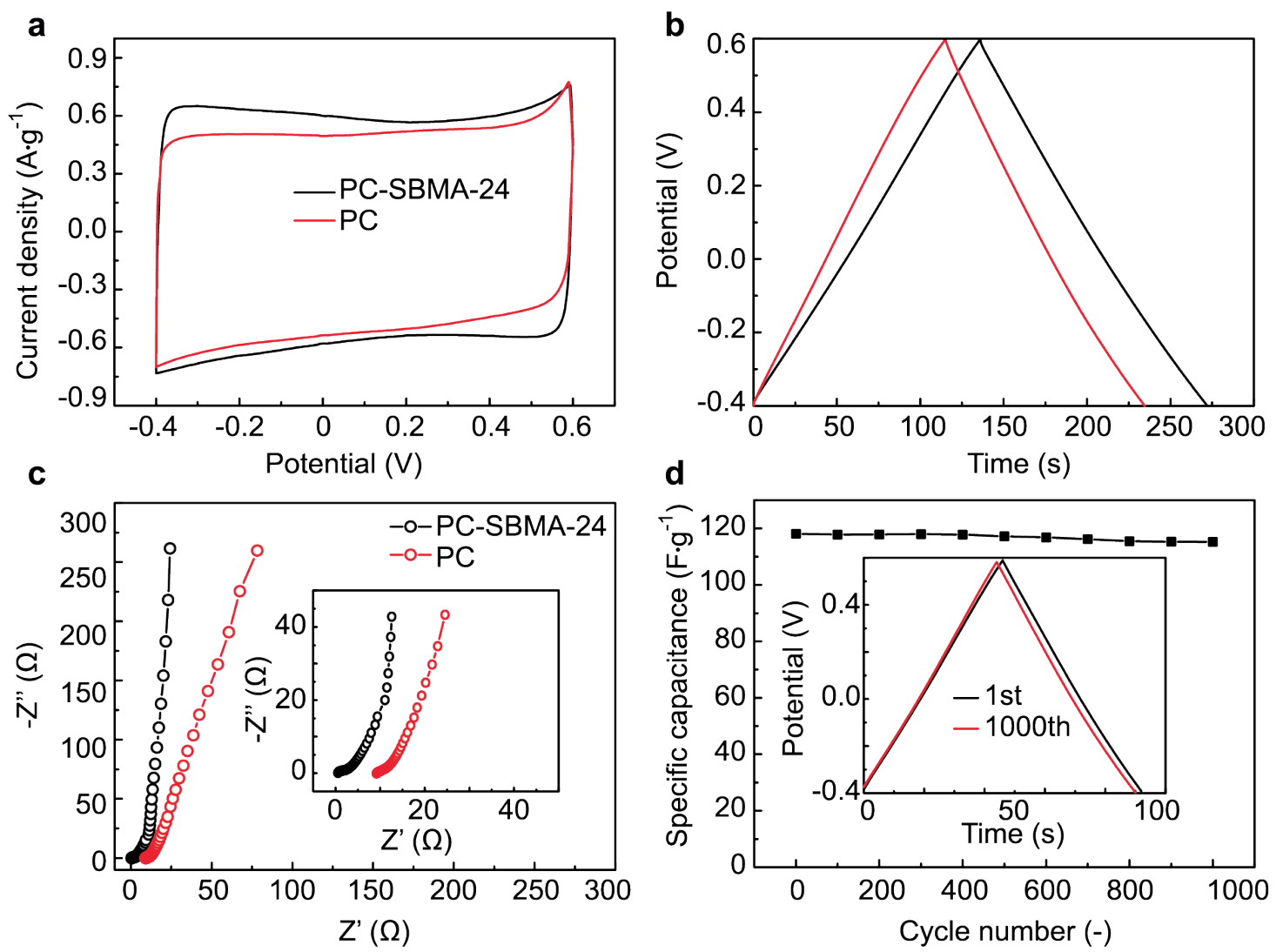

Figure 5.4. Electrochemical performance of the as-prepared material. (a) The CV curves of PC and PCSBMA-24 tested at scan rate of $5 \mathrm{mV} \cdot \mathrm{s}^{-1}$; and their (b) Galvanostatic charge/discharge curves tested at current density of $1.0 \mathrm{~A}^{-1} \mathrm{~g}^{-1}$; (c) The Nyquist plots in the frequency range of $100 \mathrm{kHz}$ to $10 \mathrm{mHz}$ (inset: Nyquist plots at high frequency range); (d) Capacitance retention plot of PC-SBMA-24 with cycle number. (inset: the first and $1000^{\text {th }}$ cycle at a constant current density of $2.5 \mathrm{~A}^{\circ} \mathrm{g}^{-1}$. All tests were conducted in $1 \mathrm{M} \mathrm{NaCl}$ aqueous deaerated solution.

The galvanostatic charge/discharge (GCD) performance of PC and PC-SBMA24 electrodes was measured at a current density of $1.0 \mathrm{~A}^{\mathrm{g}} \mathrm{g}^{-1}$. As shown in Figure $\mathbf{5 . 4} \mathbf{~ b}$, the GCD curves of the two electrodes have a classic triangular shape, indicating a typical EDLC behaviour without any Faradaic reaction. The symmetric triangular shape with linear variation of potential with time shows that these electrodes exhibit good reversibility. The rate performance of these electrodes was tested under various current densities ranging from $0.5-5.0 \mathrm{~A}^{-1} \mathbf{g}^{-1}$ (Figure S5.8 $\mathbf{c}$ and S5.8 d). All the curves display symmetrical triangular shapes, indicating excellent electrochemical reversibility, which is crucial for high efficiency and long-term operation of CDI (El-Deen et al., 2016a; Yang et al., 2014). The capacity of PC-SBMA-24 (119.4 F· $\left.\mathrm{g}^{-1}\right)$ is evidently

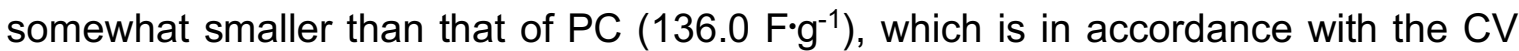
measurements. This may be due to reduction in ion adsorption that results from a zwitterionic polymer layer over the PC surface (Gao et al., 2016b). 
Electrochemical impedance spectroscopy (EIS) has been recognized as a useful measurement to clarify the ionic transportation kinetics at the interface between the electrode and the electrolyte. EIS was carried out over a frequency range of $100 \mathrm{kHz}$ to $0.01 \mathrm{~Hz}$ to investigate the ion transport behaviour and electrical resistance of the as-prepared electrode materials. In the low-frequency region, the Nyquist plots (Figure $5.4 \mathrm{c}$ ) are linear and steep. In general, the larger the slope of this part of the plot, the faster the EDL is formed (Bi et al., 2016b; Fu et al., 2014; Oschatz et al., 2014; Puthusseri et al., 2014; Rose et al., 2011). The slope for PC-SBMA-24 is smaller than that for PC, indicating slower formation of EDL due to the grafted zwitterionic polymer layer. This is in line with the CV and GCD analyses. It is interesting to note that there was nearly no semicircle in the high-frequency region, indicating ultra-small transfer resistance of both PC and PC-SBMA-24 electrodes (Bi et al., 2016b). Generally, all measurements point towards purely capacitive behavior, indicating that $P C$ and PC-SBMA-24 have excellent pore accessibility for the electrolyte. Figure $\mathbf{5 . 4} \mathbf{d}$ shows

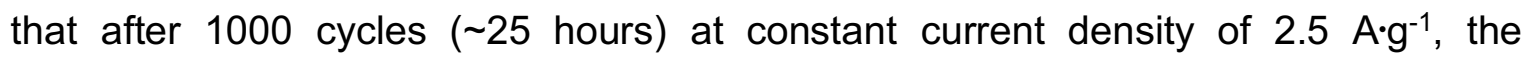
capacitance retention of PC-SBMA-24 is $97.5 \%$; such excellent durability is vital for long-term use in practice.

To estimate the electrosorption capacity of the prepared electrodes, a pair of fabricated electrodes with a thickness ca. $80 \mu \mathrm{m}$ (Figure S5.9 d and S5.9 e) were assembled in a prototype CDI cell as illustrated in Figure $\mathbf{5 . 5}$ a.

a

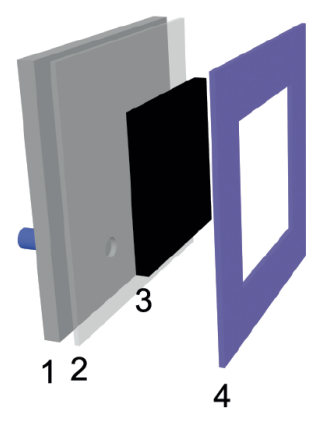

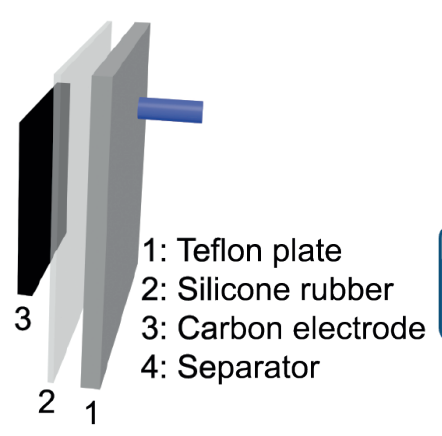

b

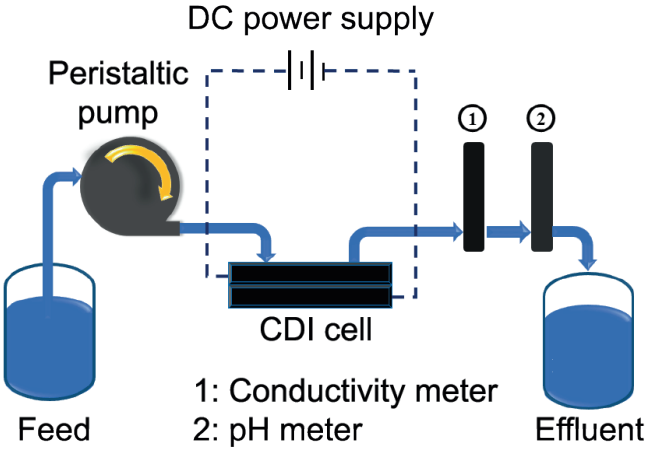

Figure 5.5. Schematic diagram of (a) Symmetrical CDI cell set up, and (b) CDI system used in this study.

The continuous mode $\mathrm{CDI}$ experiments were conducted in deaerated $\mathrm{NaCl}$ aqueous solution with a concentration of $\sim 245 \mathrm{mg}^{\cdot \mathrm{L}^{-1}}$ at applied voltage of $1.2 \mathrm{~V}$. The desalination efficiency of the PC and PC-SBMA-24 electrodes in the presence and 
absence of protein (Bovine Serum Albumin, (BSA), $100 \mathrm{mg}^{\circ} \mathrm{L}^{-1}$ ) is plotted in Figure 5.6. With only $\mathrm{NaCl}$ present, both electrodes show typical salt removal behavior, i.e., the conductivity of effluent decreased sharply under the external electrostatic field (Figure 5.7 a). This strong initial decrease in conductivity indicates a rapid adsorption ( $0.5 \mathrm{~min}$ ) of the salt ions on the electrode surface. As time passes, the concentration in the effluent then gradually recovers due to saturation of the electrode. After running the CDI tests for 100 consecutive cycles, no obvious change in cycle to cycle absorption was observed. The electrosorption capacity of both electrodes remains nearly the same (Figure $5.7 \mathrm{~b}$ ), which suggests excellent regeneration capability and chemical stability for both PC and PC-SBMA-24 electrode materials in salty water. Although the specific capacitance of PC-SBMA-24 is somewhat lower than that of pristine PC (Figure 5.4 b), its electrosorption capacity in CDI testing is almost the same as that of PC. This may be due to the superhydrophilic character imparted to the electrode surface as a result of zwitterionic polymer modification, giving better wetting of the pores and hence more complete use of the pore volume.

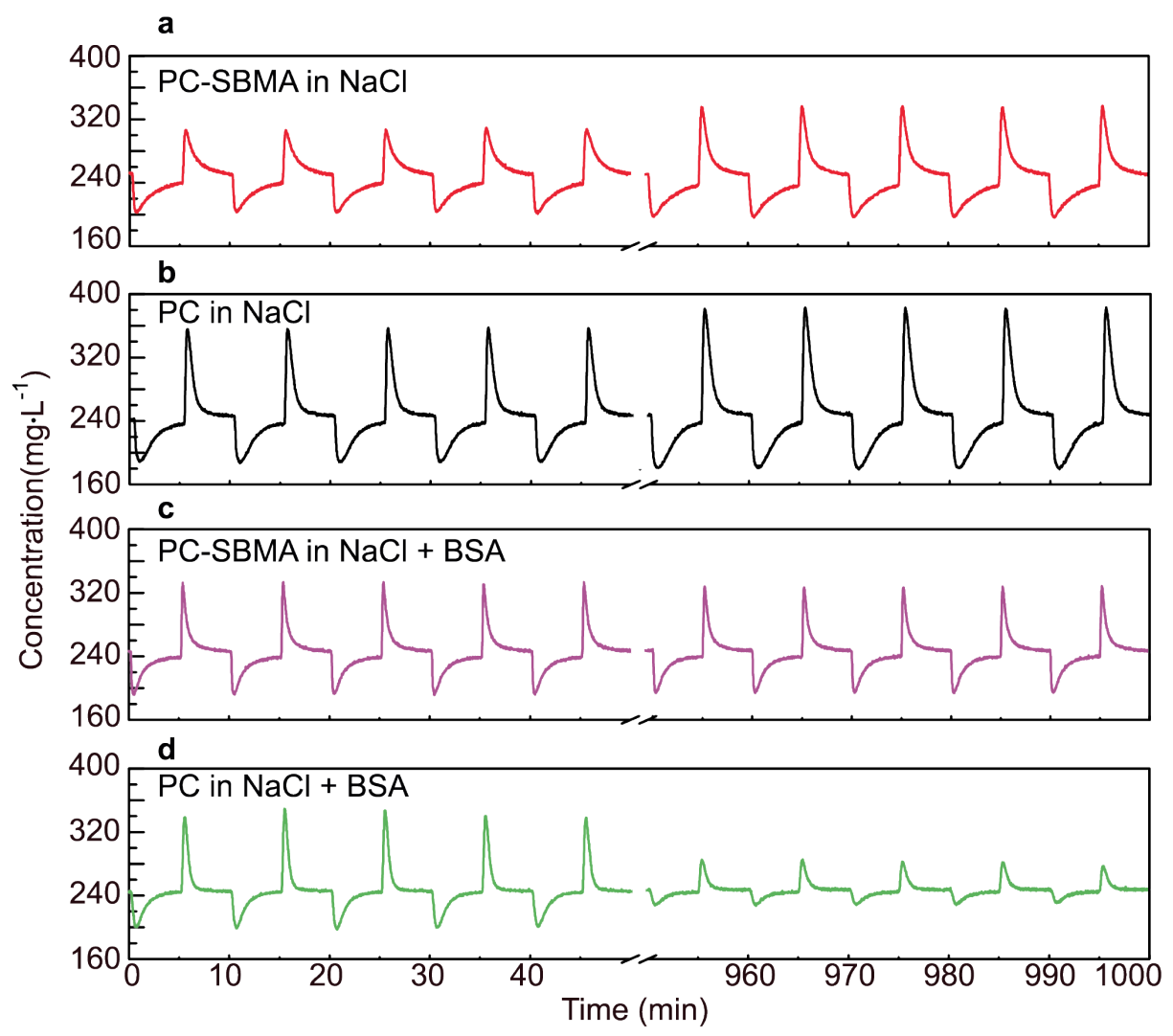

Figure 5.6. Desalination and regeneration comparison profiles of PC and PC-SBMA-24 electrode 1.2 V with flow rate of $10 \mathrm{~mL} \cdot \mathrm{min}^{-1}$ in absence $(\mathbf{a}, \mathbf{b})$ and presence $(\mathbf{c}, \mathbf{d})$ of BSA. 
Electrode fouling is a serious issue since it reduces salt removal efficiency; (Mossad and Zou, 2013) therefore, we performed a long-term salt removal test in the presence of a model foulant, BSA. Comparing the desalination and regeneration curves (c and $\mathbf{d}$ in Figure 5.6), there is systematic decline in the performance of the PC electrode after 100 cycles, its electrosorption capacity retention is $57.9 \%$ while that of PC-SBMA-24 electrode is $99.1 \%$ (Figure 5.7 b). FESEM of the electrodes after 100 cycles in the presence of BSA showed clear evidence of BSA deposition on the pristine PC electrode (Figure S5.10 b) but not on the PC-SBMA-24 electrode (Figure S5.10 a). Further evidence of the beneficial effect of polySBMA coating is seen in the superior stability of the $\mathrm{pH}$ level in the PC-SBMA-24 long-term experiment compared with the uncoated electrodes, in both the presence and absence of BSA (Figure S5.11). This effect may be attributed to a reduction of electrochemical carbon oxidation due to the presence of the polymer brushes at the anodes which produce $\mathrm{H}^{+}$, (Gao et al., 2016b) leading to a more negative charge density. The polySBMA brushes on the PC-SBMA-24 surface prevent further carbon oxidation, especially in the presence of BSA (Figure S5.11 $\mathbf{c}$ and d), leading to a more stable production of $\mathrm{H}^{+}$ during long-term CDI operation. These results indicate that the PC-SBMA-24 electrode has high electrosorption capacity and is very stable, even in the presence of fouling biomolecules. It is expected to be suitable for application for desalination of water containing these types of foulants, such as brackish surface water.
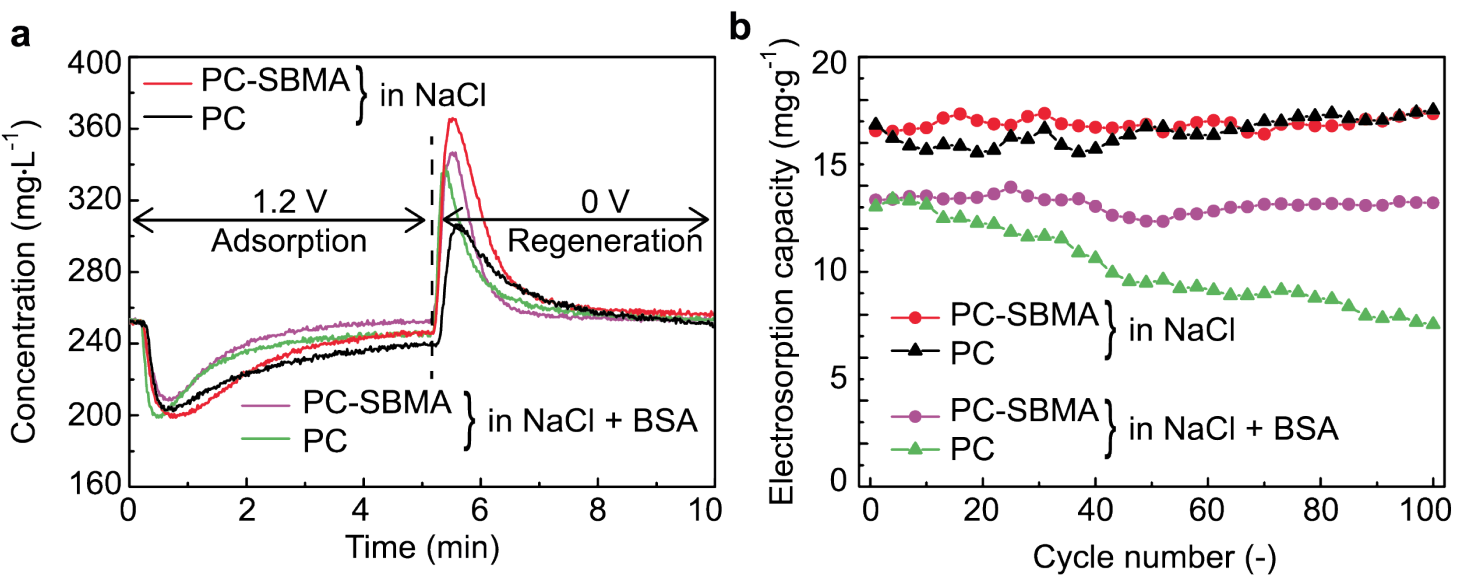

Figure 5.7. (a) First cycle of desalination and regeneration with $P C$ and PC-SBMA-24 electrode at $1.2 \mathrm{~V}$ with flow rate of $10 \mathrm{~mL} \cdot \mathrm{min}^{-1}$ in the presence and absence of BSA, (b) the electrosorption capacity retention curves over 100 cycles. 


\subsection{Conclusion}

We have developed the first zwitterionic polymer coated porous carbon electrodes with protein antifouling property and high electrosorption capacity for $\mathrm{CDI}$ applications. The PC-SBMA electrodes have electrosorption capability of $16.5 \mathrm{mg}^{-1} \mathrm{~g}^{-1}$ in $245 \mathrm{mg} \cdot \mathrm{L}^{-1} \mathrm{NaCl}$ solution, which is superior to the reported performance of other carbon-based materials (Table S5.1). Moreover, PC-SBMA-24 exhibits excellent CDI cycling stability in the presence of foulant (BSA); electrosorption capacity retention was $99.1 \%$ after 100 cycles, corresponding to an average capacity loss of $0.009 \%$ per cycle, implying $\sim 8000$ cycles for $50 \%$ capacity loss if capacity loss is exponential, or $\sim 5500$ cycles if linear. The pristine PC electrodes retained only $57.9 \%$ of their initial electrosorption capacity after 100 cycles. Clearly, the zwitterionic polymer coating contributes greatly to electrode durability, which is an essential step toward practical application in desalination. It is expected that this work not only presents a promising way to develop effective antifouling CDI electrodes with excellent electrosorption capacity for practical desalination applications, but may also have potential in other fields such as protein separation, food engineering, etc. 


\subsection{Supporting Information}

a

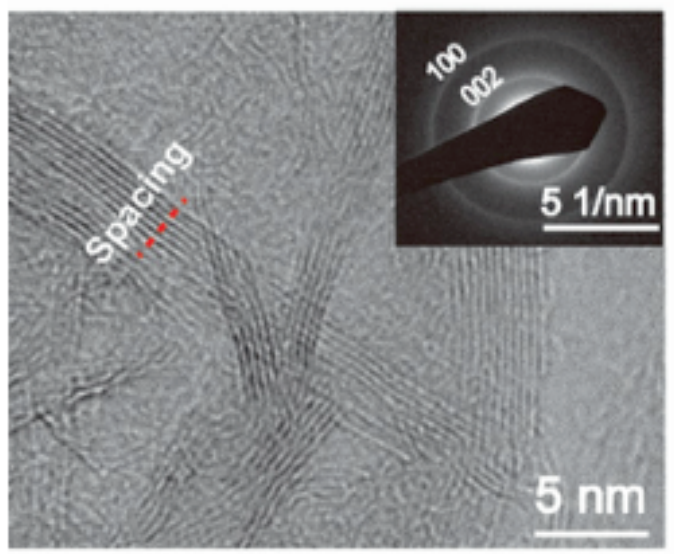

b

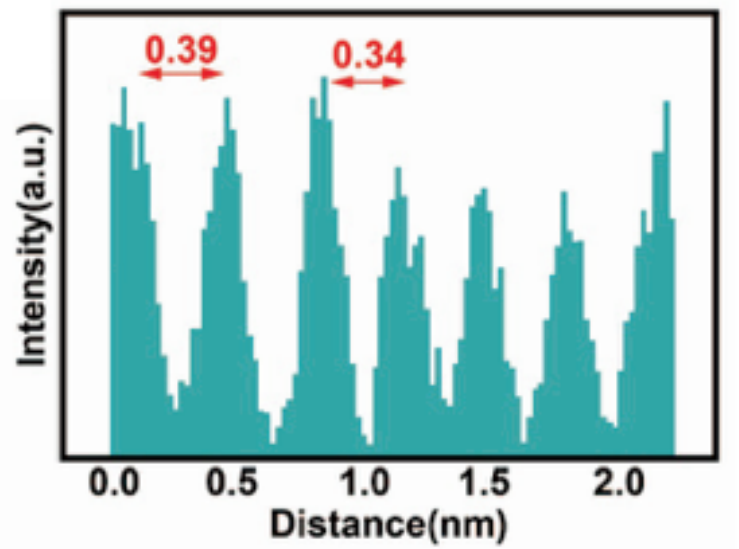

Figure S5.1. (a) High-resolution TEM image of PC, inset is the corresponding SAED pattern; (b) line profile of the d-spacing.

a

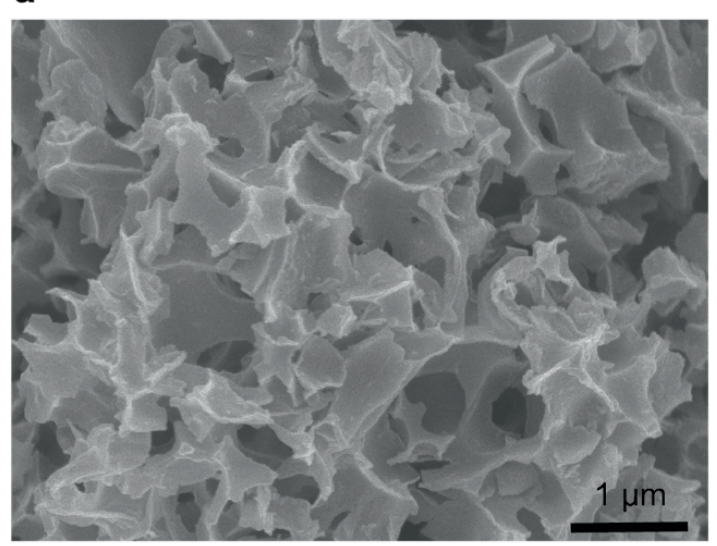

b

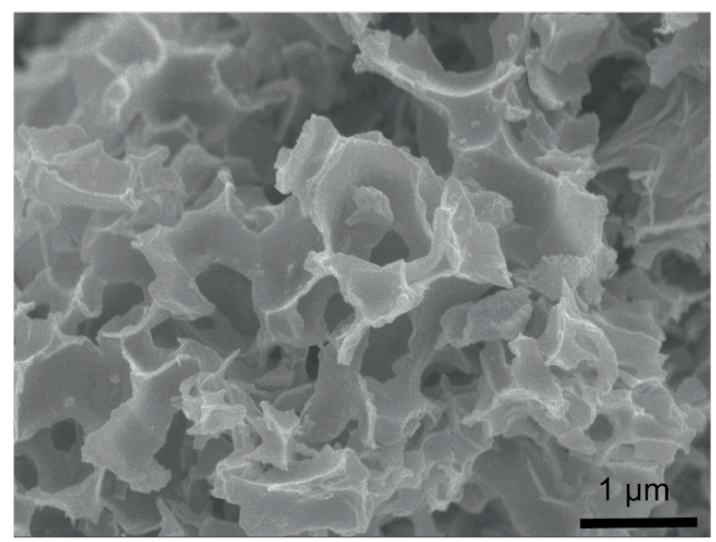

Figure S5.2. FESEM image of (a) PC-PDA, (b) PC-Br. 

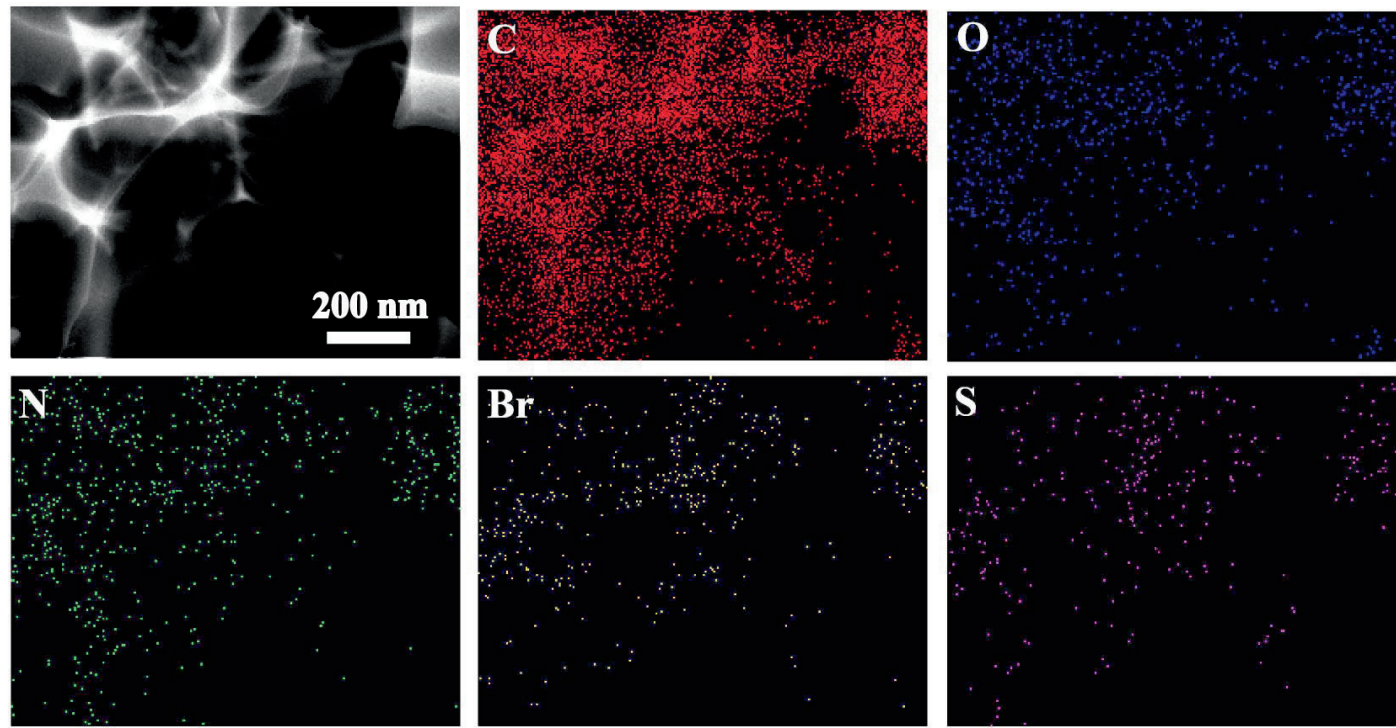

Figure S5.3. STEM of PC-SBMA-24 in dark field mode and its corresponding STEM-EDX mapping.
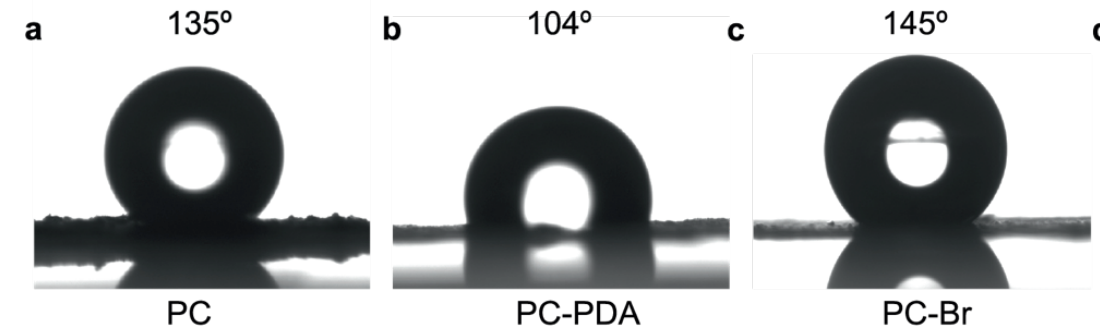

d $\quad 0^{\circ}$

e

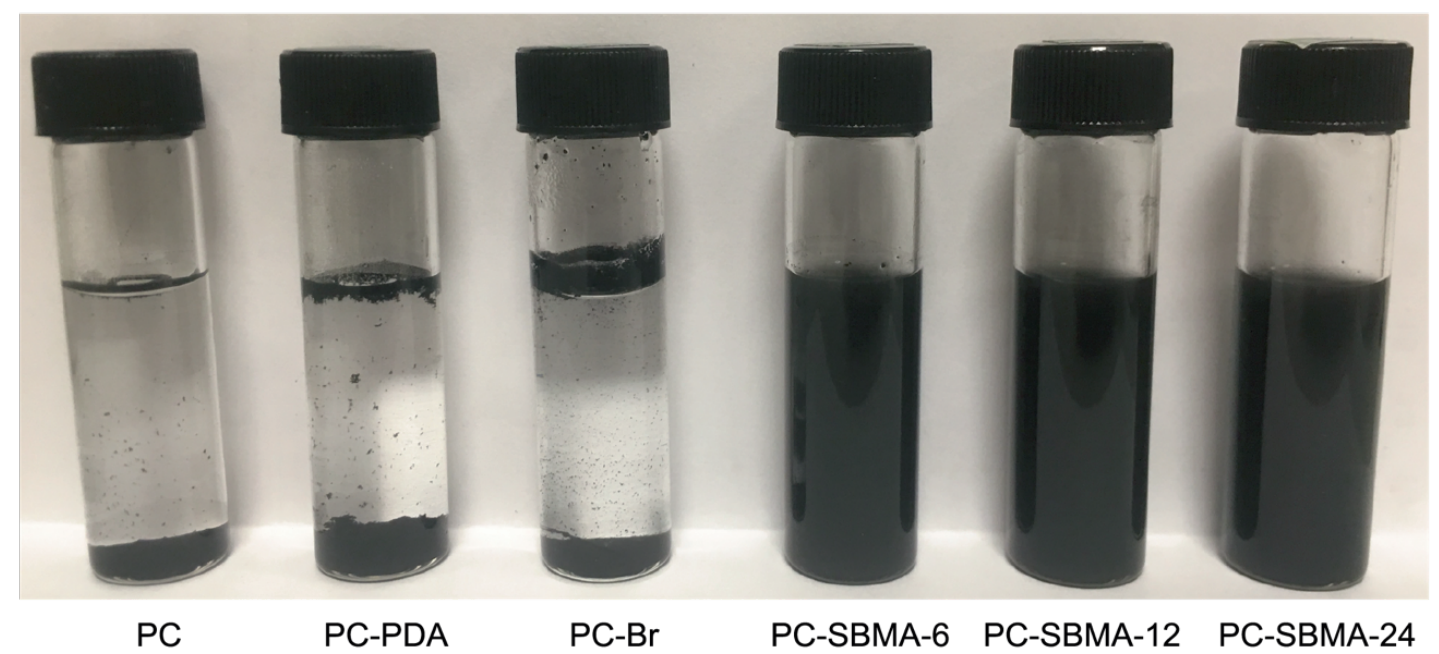

Figure S5.4. (a) - (d) Contact angles of PC, PC-PDA, PC-Br, and PC-SBMA-24, respectively; (e) visual appearance of suspensions of unmodified and modified porous carbon after three days standing. 

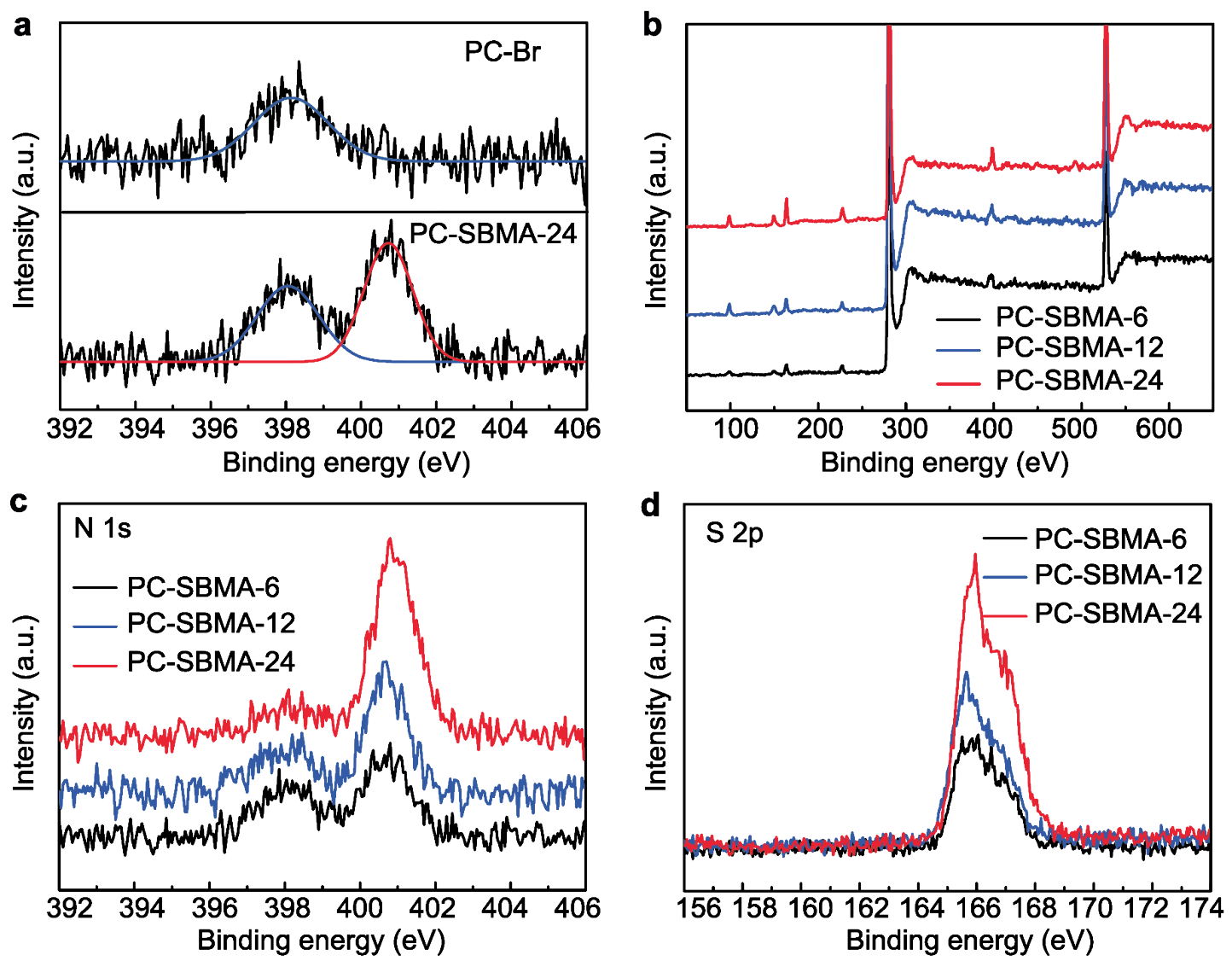

Figure S5.5. (a) High-resolution N1s comparison of PC-Br and PC-SBMA-24; (b) XPS survey spectra, high-resolution spectra of (c) N1s, and (d) S2p of PC-SBMA-6, PC-SBMA-12, and PC-SBMA-24.
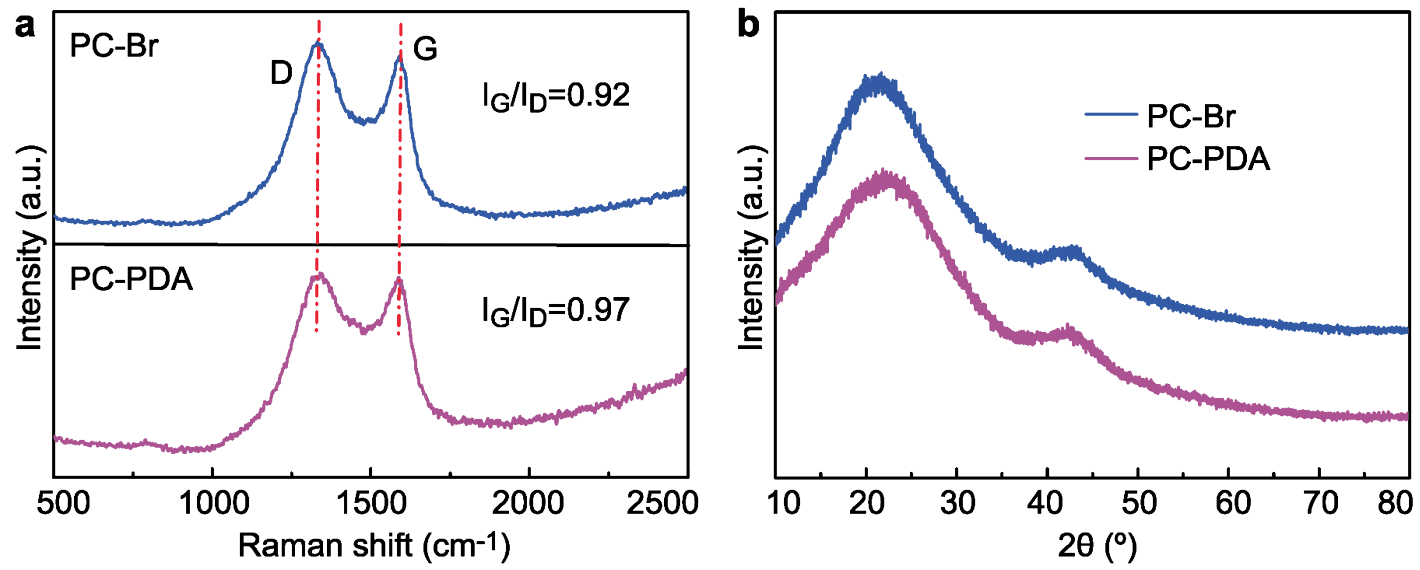

Figure S5.6. (a) Raman spectra and (b) XRD patterns of PC-PDA and PC-Br. 

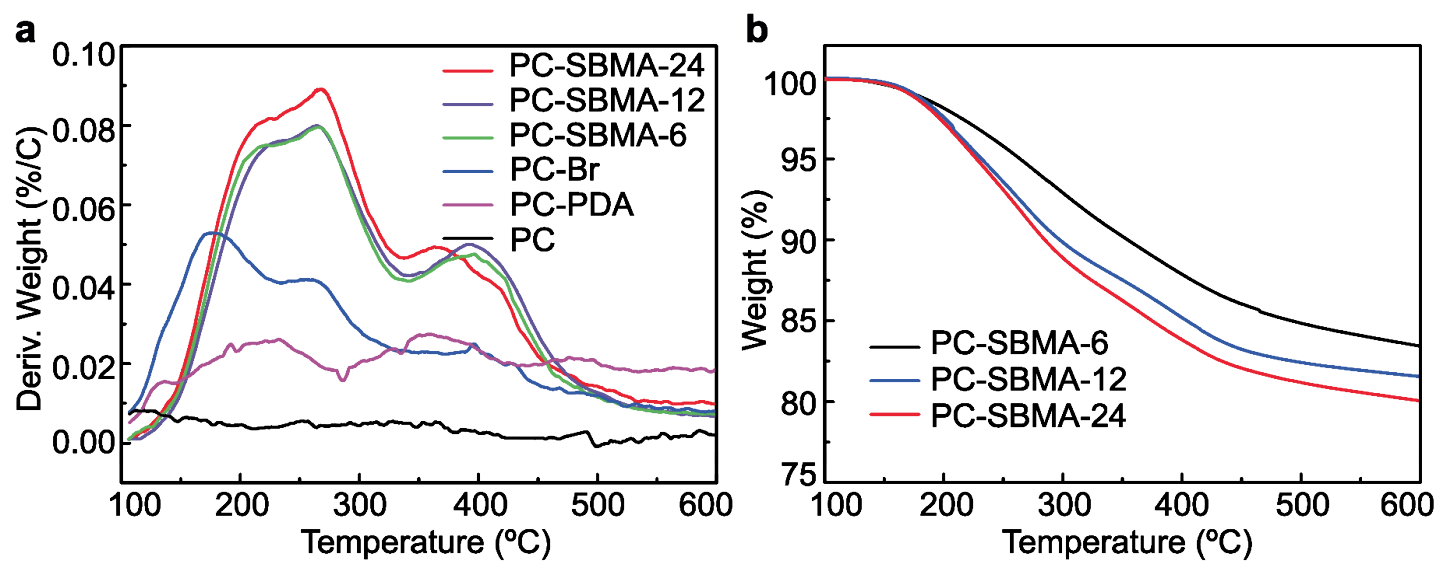

Figure S5.7. (a) DTA curves of porous carbon before and after modification; (b) TGA curves of modified porous carbon with different reaction times.
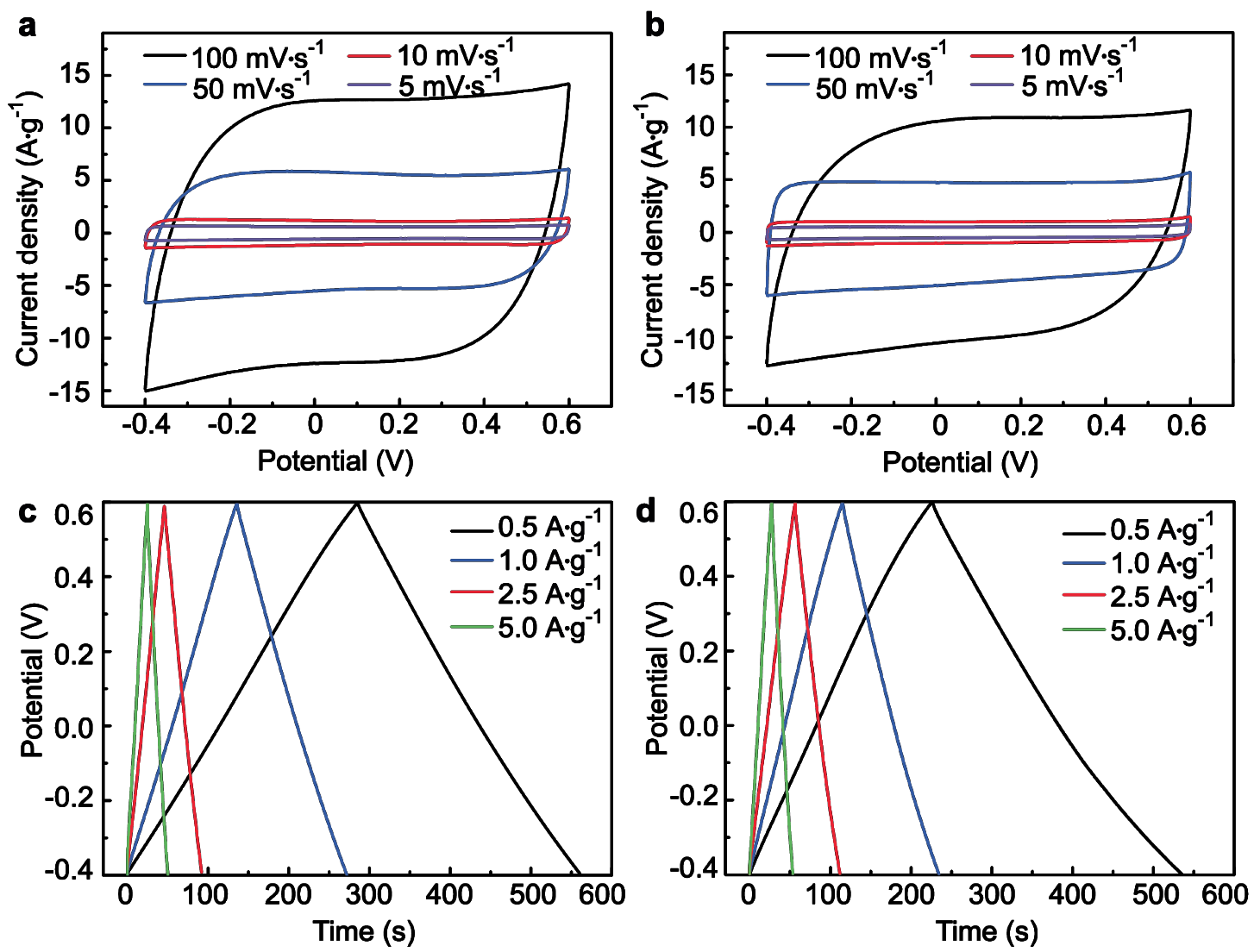

Figure S5.8. Electrochemical performance of the as-prepared material. The CV curves of PC (a) and PC-SBMA-24 (b) at different scan rates from $5 \mathrm{mV} \cdot \mathrm{s}^{-1}$ to $100 \mathrm{mV} \cdot \mathrm{s}^{-1}$; the constant current galvanostatic charge/discharge curves of PC (c) and PC-SBMA-24 (d) at different current densities from $0.5 \mathrm{~A}^{*} \mathrm{~g}^{-1}$ to $5.0 \mathrm{~A}^{\cdot} \mathrm{g}^{-1}$. All tests were conducted in $1 \mathrm{M} \mathrm{NaCl}$ aqueous deaerated solution. 

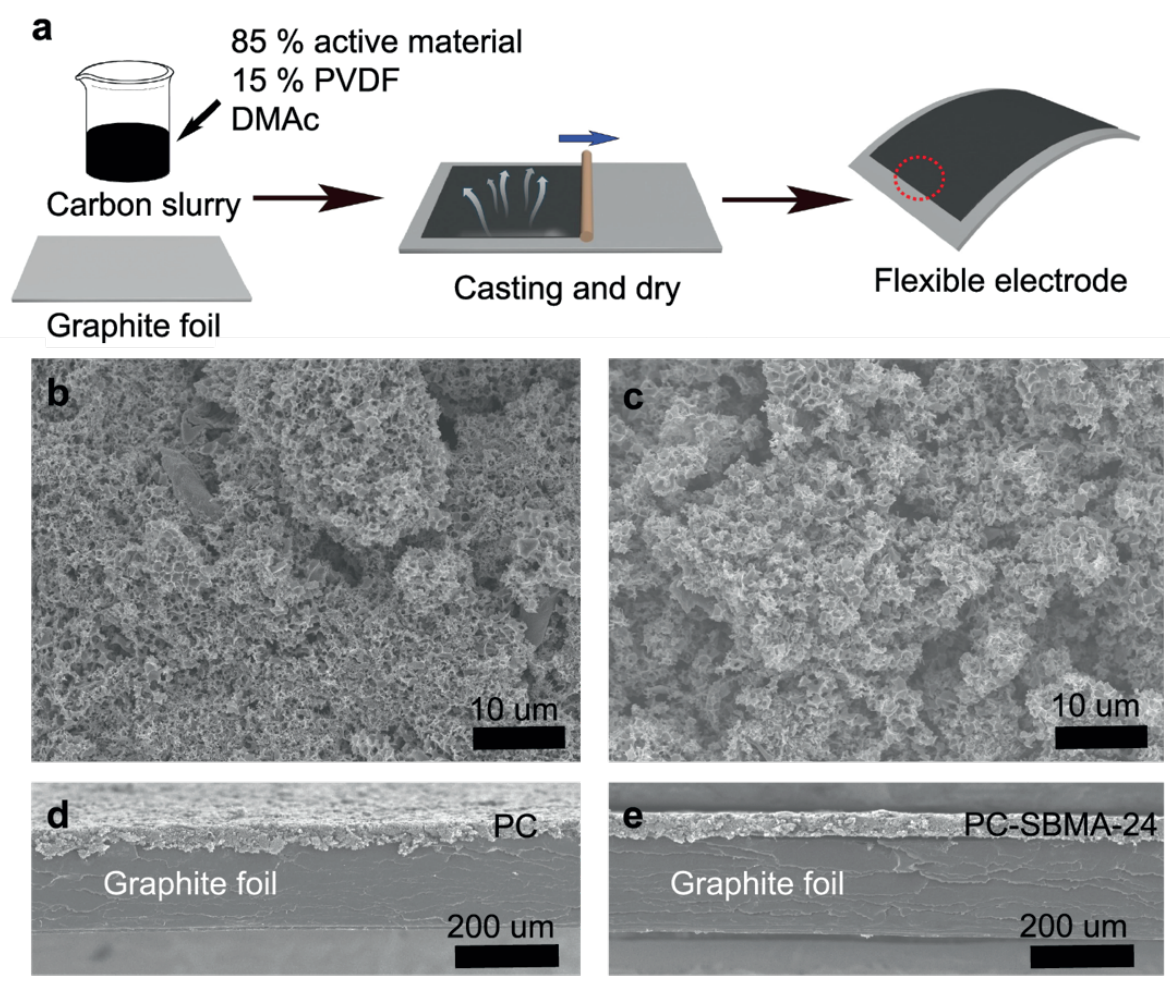

Figure S5.9. (a) Scheme of CDI electrode fabrication process; FESEM top view of (b) PC and (c) PC-SBMA-24 electrode; SEM cross-section view of (d) PC and (e) PC-SBMA-24 electrode.

a

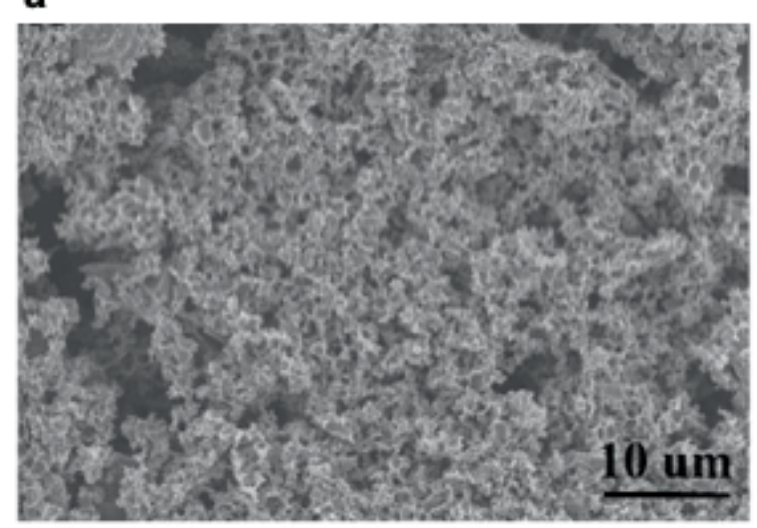

\section{b}

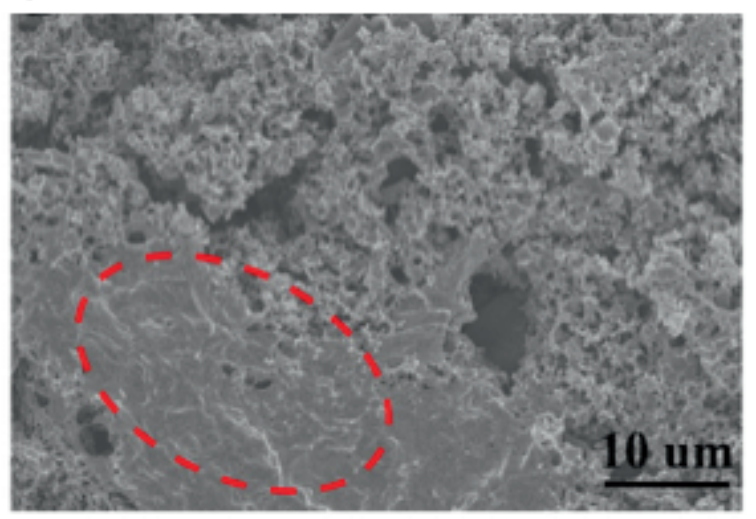

Figure S5.10. Surface comparison of (a) PC-SBMA-24 and (b) PC electrode after long-term CDI operation in the presence of BSA, the region indicated by the red dotted oval is fouled with BSA. 

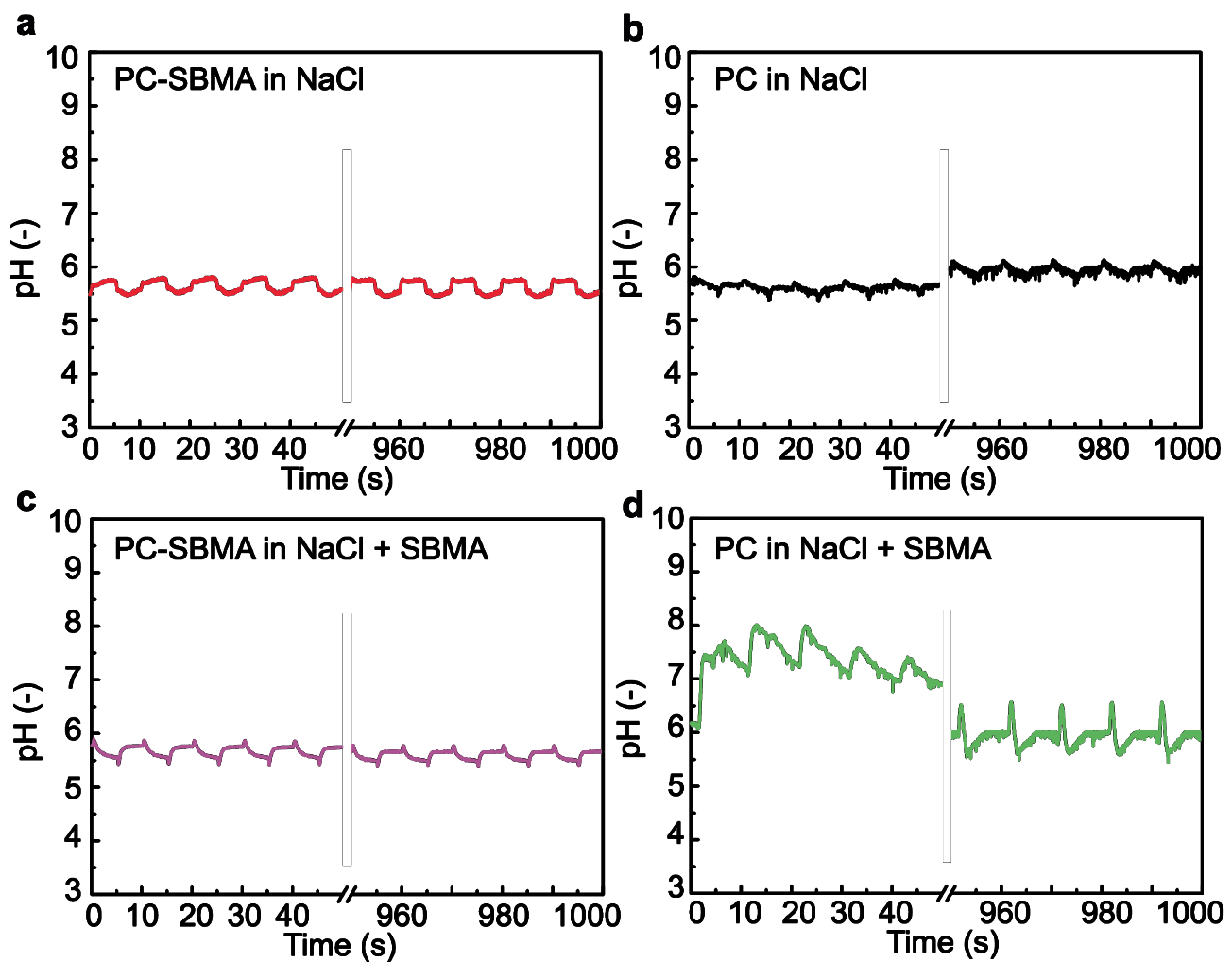

Figure S5.11. pH variation with time for different electrodes during CDI process over 100 cycles.
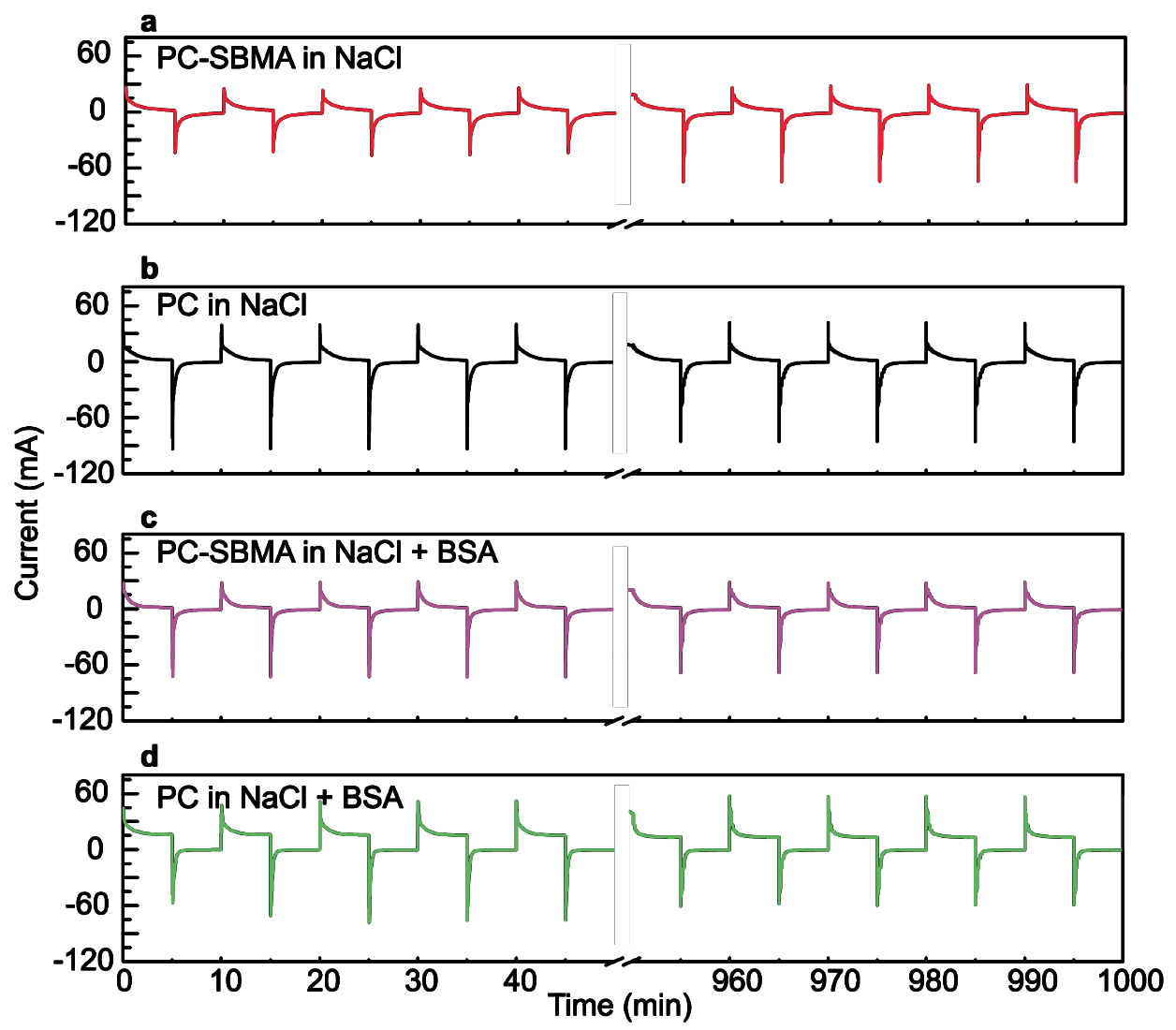

Figure S5.12. Current changes of different electrodes during CDI process over 100 cycles. 
Table S5.1. Comparison of electrosorption capacities of different carbon-based electrode materials.

\begin{tabular}{|c|c|c|c|c|c|}
\hline Materials & $\begin{array}{c}\text { Applied } \\
\text { voltage (V) }\end{array}$ & $\begin{array}{l}\text { Flow rate } \\
\left(\mathrm{mL}^{\prime} \mathrm{min}^{-1}\right)\end{array}$ & $\begin{array}{l}\text { Initial conc. } \\
\left(\mathrm{mg}^{\prime} \mathrm{mL}^{-1}\right)\end{array}$ & $\begin{array}{l}\text { Electrosorption } \\
\text { capacity }\left(\mathrm{mg}^{\cdot} \mathbf{g}^{-1}\right)\end{array}$ & Ref \\
\hline $\begin{array}{l}\text { Single-walled } \\
\text { carbon nanotubes }\end{array}$ & 2.0 & 25 & 23 & 0.75 & $\begin{array}{l}\text { (Li et al., } \\
2011)\end{array}$ \\
\hline $\begin{array}{l}\text { Amine modified } \\
\text { microporous carbon }\end{array}$ & 1.1 & 20 & 250 & 5.3 & $\begin{array}{l}\text { (Gao et al., } \\
2015 a)\end{array}$ \\
\hline $\begin{array}{l}\text { 3D macroporous } \\
\text { graphene }\end{array}$ & 2.0 & 25 & 100 & 5.39 & $\begin{array}{l}\text { (Wang et } \\
\text { al., 2013) }\end{array}$ \\
\hline $\begin{array}{l}\text { 3D porous } \\
\text { graphene }\end{array}$ & 2.0 & $\sim 10$ & 74 & 11.86 & $\begin{array}{l}\text { (Li et al., } \\
2015)\end{array}$ \\
\hline $\begin{array}{l}\text { Sponge templated } \\
\text { graphene }\end{array}$ & 1.5 & 3 & 52 & 4.95 & $\begin{array}{l}\text { (Yang et } \\
\text { al., 2014) }\end{array}$ \\
\hline $\begin{array}{l}\text { Sulfonated } \\
\text { graphene- carbon } \\
\text { nanofibers }\end{array}$ & 1.2 & 5 & 100 & 9.54 & $\begin{array}{l}\text { (Qian et al., } \\
\text { 2015) }\end{array}$ \\
\hline $\begin{array}{l}\text { N-doped porous } \\
\text { carbon }\end{array}$ & 1.2 & 15 & 40 & 15.5 & $\begin{array}{l}\text { (Zhao et } \\
\text { al., 2018) }\end{array}$ \\
\hline $\begin{array}{l}\text { Polymer coated } \\
\text { carbon cloth }\end{array}$ & 1.2 & 80 & 409 & 7.6 & $\begin{array}{l}\text { (Gao et al., } \\
2016 b)\end{array}$ \\
\hline PC & 1.2 & 10 & 245 & 16.8 & This work \\
\hline PC-SBMA-24 & 1.2 & 10 & 245 & 16.5 & This work \\
\hline
\end{tabular}





\section{CHAPTER 6}

\section{STEERING PROTEIN AND SALT ADSORPTION AND DESORPTION BY AN ELECTRICAL SWITCH APPLIED TO TAILOR MADE ELECTRODES}

We make use of spontaneous adsorption of proteins to solid electrodes that we reverse by applying an electric field to regenerate the interface. This allows us to ad- and desorb proteins to and from an electrode surface, following an inverted capacitive deionization scheme (adsorption at $0 \mathrm{~V}$; desorption at $-1.2 \mathrm{~V}$ ), leading to protein adsorption when salt is desorbing, and vice versa. We demonstrate that the incorporation of negatively charged polystyrene sulfonate (PSS) or positively charged polydiallyldimethylammonium chloride (PDMAC) in or on top of the respective electrodes increases the amount of exchanged protein from 1 to $10 \mathrm{mg} \cdot \mathrm{g}^{-1}$. Simultaneous salt exchange remains at values comparable to conventional activated carbon electrodes $\left(3 \mathrm{mg}^{\cdot} \mathrm{g}^{-1}\right)$ when polyelectrolytes are located on top of the electrodes. Besides this, an enrichment in $\beta$-lactoglobulin could be achieved starting from whey protein isolate. These are all important steps toward electrochemical technologies for protein separation, and fractionation, that do not require solvent use, and may include concurrent desalting.

This chapter has been submitted as:

P. A. Fritz, P. Zhang, T. Bruschinski, S. Sahin, L. de Smet, M. B. Chan-Park, R. M. Boom, and K. Schroën. Steering protein and salt ad- and desorption by an electrical switch applied to tailor made electrodes. 


\subsection{Introduction}

Proteins are an essential component in human nutrition and are also important in medicine and biotechnology (Chang, 1977; Geissler and Powers, 2012; Walsh, 2002). Protein losses are high in the global food system, partly due to inefficient processing (Alexander et al., 2017). The utilization of byproducts and waste streams (biorefinery) can reduce their environmental impact (Asghar et al., 2011; Galanakis, 2012; van der Goot et al., 2016; Jayathilakan et al., 2012), but only if this can be done in a sustainable, energy efficient way.

Current protein separation processes revolve mostly around filtration, (ultra)centrifugation, precipitation and chromatography (Gräslund et al., 2008; Hilbrig and Freitag, 2003; Narayanan, 1994; Scopes, 1994). Filtration and centrifugation generally do not affect protein structure, although high selectivity between components is rarely possible, whereas precipitation aggregates proteins, which influences their functionality. In terms of purity, chromatography is the best option, although desorption of the protein and regeneration of the column requires extreme conditions, and may lead to changes in protein functionality. Most desorption strategies target the solution conditions, e.g. by changing the $\mathrm{pH}$ (Tarhan and Harsa, 2014) or the ionic strength (Neyestani et al., 2003) of the eluent and thus impact the charge of the proteins, or their screening length (Gerberding and Byers, 1998). This leads to high eluent usage and consequently high environmental impact, especially in large scale, preparative chromatography (Coskun, 2016; Cuatrecasas, 1970; Fuciños et al., 2019; Pedersen et al., 2003; Simon et al., 1996).

We here investigate the use of electrochemical stimuli to cycle surface properties from adsorptive to desorptive, without requiring external chemicals. By applying either positive or negative potential, interfacial properties such as surface charge, double layer structure and oxidative state can be altered, which influences the ad- and desorption behavior of ions and molecules (Maharjan et al., 2008). This strategy has mostly been tested for small species (ions) in capacitive deionization (Suss et al., 2015; Tang et al., 2019) and aromatic sulfonates or corticosteroids in electrochemically modulated liquid chromatography and related processes (Collins and Arrigan, 2009; Harnisch and Porter, 2001; Porter and Takano, 2000; Su and Hatton, 2017a; Yamini et al., 2014). The extension to proteins (Mu et al., 2007; Su et 
al., 2017a) and the investigation of the underlying force modulations (Barten et al., 2003a) at the interface have been limited so far, but are crucial for further development towards sustainable protein separation processes. Here we propose for the first time an inverted capacitive protein separation process that allows the selective isolation of protein via adsorption to and desorption from electrodes that are coated with polyelectrolytes and to which a potential bias can be applied to induce desorption.

\subsection{Materials and Methods}

\subsubsection{Chemicals}

The following chemicals and materials were purchased from Merck (The Netherlands): activated carbon (AC, particle size $\sim 10 \mu \mathrm{m})$, poly-(vinylidene fluoride) (PVDF, 534,000 $\mathrm{g} \cdot \mathrm{mol}^{-1}$ ), dimethylacetamide (DMAc), nitric acid $(70 \%)$, ethylenediamine (99\%), poly(sodium 4-styrenesulfonate) (PSS, 1,000 kg.mol-1), polydiallyldimethylammonium chloride (PDADMAC, 400-500 $\mathrm{kg} \cdot \mathrm{mol}^{-1}$ ), and sodium chloride ( $>99.5 \%$, Merck, The Netherlands). Furthermore styrene butadiene rubber (Zeon, Japan) and whey protein isolate (Biopro, Davisco, USA) were used. Water in all experiments was deionized, and purified with a Merck Millipore system $(18.2 \mathrm{M} \Omega \cdot \mathrm{cm})$.

\subsubsection{Electrode preparations}

Various electrodes were prepared, as described in the next sections and summarized in Table 6.1.

\subsubsection{Activated carbon electrodes}

Most electrodes were based on AC electrodes. To prepare these, activated carbon powder was mixed with 10 wt\% PVDF binder in DMAc overnight and cast onto graphite foil using a rod coater with a $400 \mu \mathrm{m}$ gap. Subsequently electrodes were dried over night at room temperature. All electrodes had circular shape with $5 \mathrm{~cm}$ diameter (Fritz et al., 2018). 


\subsubsection{Surface modified activated carbon electrodes}

To increase the negative charges on the surface of the AC powder, $10 \mathrm{~g} \mathrm{AC}$ was oxidized by exposing it to concentrated nitric acid (70\%) for $24 \mathrm{~h}$ (AC-). Subsequently the AC- powder was washed with around 1 I of water. To obtain positive surface charges on the activated carbon $\left(\mathrm{AC}^{+}\right), \mathrm{AC}^{-}$was suspended in ethylenediamine at $120{ }^{\circ} \mathrm{C}$ until all the liquid was evaporated (Gao et al., 2015a, 2016a). Electrodes were made from these materials using PVDF binder as described above.

\subsubsection{Dip-coated activated carbon electrodes}

To prepare dip-coated electrodes (White et al., 2016) (Dip ${ }^{+}$and Dip-), unmodified $A C$ electrodes were immersed for $15 \mathrm{~min}$ in $20 \mathrm{mM}$ (based on $\mathrm{Mw}$ of monomer) PDADMAC or PSS solution, each containing $100 \mathrm{mM} \mathrm{NaCl}$. Subsequently, the electrodes were immersed for $1 \mathrm{~h}$ in water and dried.

\subsubsection{Composite polyelectrolyte-activated carbon electrodes}

These electrodes (Comp ${ }^{+}$and Comp-) were prepared by mixing 5 wt $\%$ of PDADMAC or PSS, respectively, with AC powder and styrene butadiene rubber as binder in water. This slurry was then cast onto graphite foil (Fritz et al., 2019a). Like the $A C$ based electrodes, these electrodes were circular with a diameter of $5 \mathrm{~cm}$.

Table 6.1. Electrode combinations and abbreviations

\begin{tabular}{|c|c|c|}
\hline Anode & Cathode & Abbreviation of combination \\
\hline Activated carbon (AC) & Activated carbon (AC) & $A C$ \\
\hline $\begin{array}{l}\text { Nitric acid treated activated } \\
\text { carbon }\left(\mathrm{AC}^{-}\right)\end{array}$ & $\begin{array}{l}\text { Ethylenediamine treated activated } \\
\text { carbon }\left(\mathrm{AC}^{+}\right)\end{array}$ & $\mathrm{AC}^{+/-}$ \\
\hline $\begin{array}{l}\text { PSS mixed into the electrode } \\
\text { slurry (Comp-) }\end{array}$ & $\begin{array}{l}\text { PDADMAC mixed into the } \\
\text { electrode slurry }\left(\text { Comp }{ }^{+}\right)\end{array}$ & Comp $^{+/-}$ \\
\hline $\begin{array}{l}\text { AC electrode dip coated with } \\
\left.\text { PSS (Dip }{ }^{-}\right)\end{array}$ & $\begin{array}{l}\text { AC electrode dip coated with } \\
\text { PDADMAC }\left(\text { Dip }^{+}\right)\end{array}$ & $\mathrm{Dip}^{+/-}$ \\
\hline
\end{tabular}

\subsubsection{Characterization}

X-ray photoelectron spectroscopy (XPS) was used to study the chemical composition of the electrodes. In addition to wide scans, C1s, O1s, N1s and S2p narrow scans were also measured. Thermogravimeteric analysis (TGA) was 
conducted under nitrogen atmosphere after fully drying the samples, to determine the composition of the electrodes.

\subsubsection{Electrochemical measurements}

To determine the potential of the zero charge of all electrodes, cyclic voltammetry measurements were conducted at $0.5 \mathrm{mV} \cdot \mathrm{s}^{-1}$ within a potential window of -1 to $1 \mathrm{~V}$ (Gao et al., 2018).

\subsubsection{Capacitive separation measurements}

The ad- and desorption of proteins and ions in response to capacitive current was measured using a flow through capacitor system. Anode/cathode combinations, separated by a piece of nylon cloth, were assembled in a flow cell that was created by the technical workshop of our university, as described in previous articles (Fritz et al., 2018, 2019a). A solution containing $1.5 \mathrm{mg}^{\circ} \mathrm{g}^{-1}$ whey protein isolate (WPI) and $0.3 \mathrm{mg} \cdot \mathrm{g}^{-1}$ sodium chloride was continuously flushed between the electrodes at $1 \mathrm{~mL} \cdot \mathrm{min}^{-1}$ and the applied potential was switched between $0 \mathrm{~V}$ and $-1.2 \mathrm{~V}$ during 10 cycles. The time for each step was varied between 300 and $1800 \mathrm{~s}$.

The gravimetric ad- and desorption capacity of salt (gSAC and gSDC, respectively) and proteins (gPAC and gPDC, respectively) were determined for cycles 3 to 10 , based on the equations below,

$$
\begin{array}{r}
g S A C=\frac{m_{s, a d s}}{m_{e}} \\
g S D C=\frac{m_{s, d e s}}{m_{e}} \\
g P A C=\frac{m_{\text {prot }, a d s}}{m_{e}} \\
g P D C=\frac{m_{\text {prot,des }}}{m_{e}}
\end{array}
$$

where $m$ is the mass of salt (s), or protein (prot) adsorbed (ads) or desorbed (des), respectively; $m_{e}$ is the average mass of active material on the electrodes $(0.186 \mathrm{~g})$. 


\subsubsection{HPSEC measurements}

Whey protein isolate consists of a number of proteins. The amounts of $\beta$-lactoglobulin, $\alpha$-lactalbumin, and bovine serum albumin in the outflow of the electrochemical flow cell were determined using high performance size exclusion chromatography (HPSEC, Thermo Dionex Ultimate 3000 UHPLC, Thermo Fisher Scientific, USA) using two columns (TSKGel G3000SWXL 5um 300x7.8 mm and TSKGel G2000SWXL 5um 300x7.8 mm). As eluent, $30 \%$ acetonitrile in ultrapure water with $0.1 \%$ trifluoroacetic acid was used at a flowrate of $1.5 \mathrm{~mL} \cdot \mathrm{min}^{-1}$ and $30^{\circ} \mathrm{C}$. The UV detection wavelength was set to $214 \mathrm{~nm}$.

\subsection{Results and discussion}

In this section, we first present the composition and characteristics of the electrodes to which the components (protein and salt) adsorb. We then illustrate various process conditions, and discuss the differences in ad- and desorption behavior, leading to suggestions for innovative separation design.

We compare four electrode combinations all based on activated carbon as shown in Table 6.1: (1) two AC electrodes, (2) an anode with carboxylic groups (AC-) and a cathode with ethylenediamine $\left(\mathrm{AC}^{+}\right)$to create a net-positive charge when exposed to water at neutral pH (Gao et al., 2015a). Additional electrodes were made with charged polymers (PSS or PDADMAC) that were either (3) dip-coated onto unmodified AC electrodes or were cast as an integral part of the electrode slurry (4).

Figure 6.1 a and Table 6.2 indicate that the AC- electrodes were successfully modified with carboxylic groups, as evident from the larger oxygen content compared to the $A C$ electrode, and the shift in the potential of zero charge (PZC) to more positive values (Figure 6.1 d). The N1s peak at $399 \mathrm{eV}$ (Figure $6.1 \mathrm{~b}$ and Table 6.2) of the ethylenediamine treated electrodes $\left(\mathrm{AC}^{+}\right)$proves the presence of amine groups, which shifted the PZC to negative values (Figure 6.1 d), as found by Gao et al. (2015a). The electrodes containing PSS obtained by dip coating or as integral part of the matrix, show prominent S2p peaks at $168 \mathrm{eV}$ due to the sulfonate groups (Figure $6.1 \mathrm{c}$ ), whereas the PDADMAC containing electrodes have N1s peaks at 399 and $402 \mathrm{eV}$ for $\mathrm{C}-\mathrm{NH}_{2}$ and $\mathrm{C}_{4}-\mathrm{N}^{+}$, respectively (Pei and Lucy, 2014) (Figure $6.1 \mathrm{~b}$ and Table 6.2). As described earlier for modified AC electrodes, also here a shift in PZC was observed; 
negatively charged polymers lead to more positive PZC, and positively charged polymers to more negative values (Figure 6.1 d). Similar PZC values were obtained in earlier work using impedance spectroscopy (Fritz et al., 2019a). The shift in PZC is less pronounced compared to $\mathrm{AC}^{+}$and $\mathrm{AC}^{-}$electrodes without polymers, since the majority of the incorporated polymer is not located directly at the interface of the activated carbon and thus influences the external electric double layer less strongly.
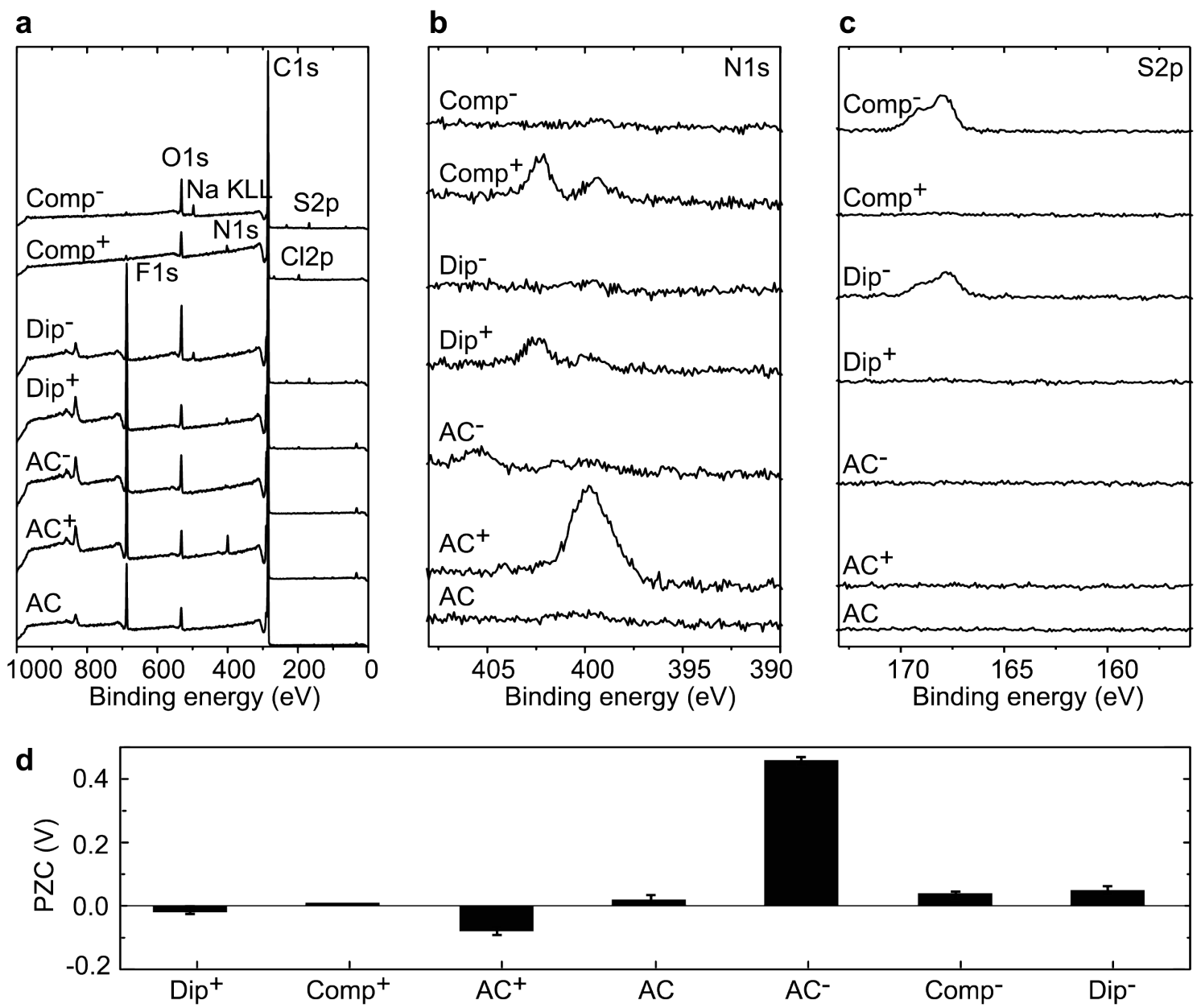

Figure 6.1. X-ray photoelectron spectra of all electrodes as indicated in the plots. a wide scan. $\mathbf{b}$ Highresolution spectrum of N1s and c S2p. d Potential of zero charge of all electrodes (CV scans are shown in Figure S6.3).

Thermogravimetric analysis indicates on average $7.7 \mathrm{wt} \%$ (Figure $\mathbf{S 6 . 2}$ and Table 6.3) weight loss for Comp- and Comp ${ }^{+}$, when heated to $600{ }^{\circ} \mathrm{C}$. This is slightly less compared to the ratios used to prepare the electrode slurry ( $5 \mathrm{wt} \%$ binder and $5 \mathrm{wt} \%$ polymer). The weight loss of all AC electrodes is around $10.6 \mathrm{wt} \%$, which is in 
line with the 10 wt\% PVDF used for the electrode preparation, while Dip ${ }^{+}$and Dip- lose $12.4 \mathrm{wt} \%$ of their mass, which corresponds to $1.6 \mathrm{wt} \%$ polymer content.

Table 6.2. Elemental analysis (\% atomic composition) of X-ray photoelectron spectra of different electrodes. Two distinct $\mathrm{N}$ peaks were found at 1) $399 \mathrm{eV}$ and 2) $402 \mathrm{eV}$ for $\mathrm{C}-\mathrm{NH}_{2}$ and $\mathrm{C}_{4}-\mathrm{N}^{+}$, respectively.

\begin{tabular}{|c|c|c|c|c|c|c|c|c|}
\hline Element & Position (eV) & AC & $\mathrm{AC}^{+}$ & $\mathrm{AC}^{-}$ & Dip $^{+}$ & Dip $^{-}$ & Comp ${ }^{+}$ & Comp- \\
\hline C1s & 285 & 85.3 & 77.3 & 80.1 & 78.6 & 79.8 & 92.1 & 79.8 \\
\hline $01 \mathrm{~s}$ & 532 & 5.9 & 4.3 & 6.1 & 9.8 & 8.8 & 5.3 & 11.6 \\
\hline F1s & 687 & 8.8 & 14.0 & 13.8 & 6.7 & 7.7 & - & - \\
\hline \multirow{2}{*}{ N1s } & 1) 399 & \multirow{2}{*}{ - } & \multirow{2}{*}{ 1) 4.4} & \multirow{2}{*}{ - } & 1) 1.2 & \multirow{2}{*}{-} & 1) 0.6 & \multirow{2}{*}{-} \\
\hline & 2) 402 & & & & 2) 3.2 & & 2) 1.0 & \\
\hline $\mathrm{Cl} 2 \mathrm{p}$ & 197 & - & - & - & - & - & 1.0 & - \\
\hline $\mathrm{S} 2 \mathrm{p}$ & 168 & - & - & - & - & 1.5 & - & 2.9 \\
\hline $\mathrm{Na} \mathrm{KLL}$ & 497 & - & - & - & - & 2.2 & - & 5.7 \\
\hline $\mathrm{O} / \mathrm{C}$ & & 0.07 & 0.06 & 0.08 & 0.12 & 0.11 & 0.06 & 0.15 \\
\hline $\mathrm{N} / \mathrm{C}$ & & - & 0.06 & - & 0.06 & - & 0.02 & - \\
\hline $\mathrm{S} / \mathrm{C}$ & & - & - & - & - & 0.02 & - & 0.04 \\
\hline $\mathrm{F} / \mathrm{C}$ & & 0.10 & 0.18 & 0.17 & 0.09 & 0.10 & - & - \\
\hline
\end{tabular}

Table 6.3. Thermogravimetric weight loss after heating sample from 100 to $600{ }^{\circ} \mathrm{C}$.

\begin{tabular}{lcc}
\hline Material & Weight loss (wt\%) & Estimated polymer content (wt\%) \\
\hline $\mathrm{AC}$ & 10.6 & 0 \\
\hline $\mathrm{AC}^{+}$ & 10.8 & 0 \\
\hline $\mathrm{AC}^{-}$ & 10.5 & 0 \\
\hline $\mathrm{Dip}^{+}$ & 12.4 & 1.8 \\
\hline $\mathrm{Dip}^{-}$ & 12.1 & 1.5 \\
\hline Comp $^{+}$ & 7.8 & 2.8 \\
\hline Comp $^{-}$ & 7.5 & 2.5 \\
\hline
\end{tabular}

When testing the different electrode combinations in the flow cell, protein adsorption was detected in the passive phase during which $0 \mathrm{~V}$ was applied (minimum UV absorbance in outgoing fluid). In contrast, during the active phase when a potential of $-1.2 \mathrm{~V}$ was applied, an increase in UV signal indicated protein desorption (Figure 6.2 a). At the same time salt ad- and desorption occurred vice versa: while proteins adsorbed, salt was released and while proteins desorbed, salt was stored in the electrodes as indicated in Figure 6.2 b and $\mathbf{c}$. In the following, we will first discuss 
protein separation and subsequently continue with more insights about the salt interchange.

For Comp ${ }^{+/-}$and $\mathrm{Dip}^{+/-}$electrodes, the amount of ad- and desorbed protein of about $10 \mathrm{mg} \cdot \mathrm{g}^{-1}$, is much larger than that of, about $1 \mathrm{mg} \cdot \mathrm{g}^{-1}$, for the $\mathrm{AC}$ and $\mathrm{AC}^{+/-}$ electrodes (Figure 6.3 a), with stable values for cycles 3 to 10 (Figure S6.4-S6.7). The whey proteins have an overall negative charge at the given conditions leading to storing at interfaces with a positive charge. The cathode carrying PDADMAC can story more proteins than the standard $\mathrm{AC}^{+}$electrodes due to the presence of the polymer. Furthermore, the interaction strength between the polymer and protein is weaker than that with activated carbon, and thus proteins detach more readily. This protein exchange also takes place at lower potential, but lower amounts can be seperated (e.g. $-0.4 \mathrm{~V}$ leads to $3 \mathrm{mg}^{\cdot} \mathrm{g}^{-1}$ on average (Figure S6.8)).

a

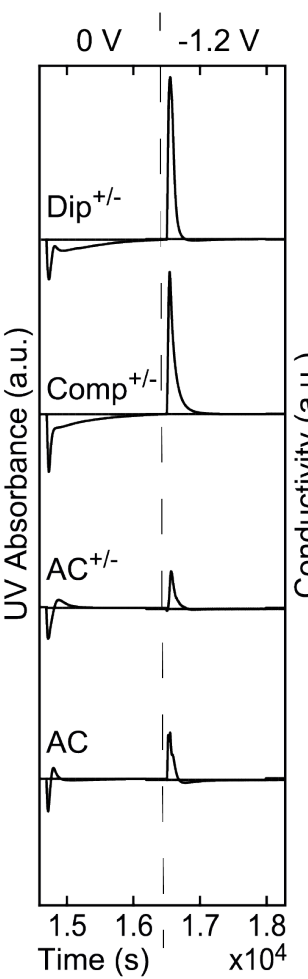

b

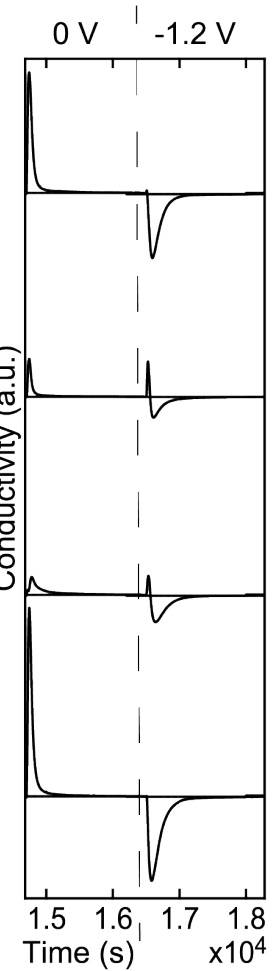

C

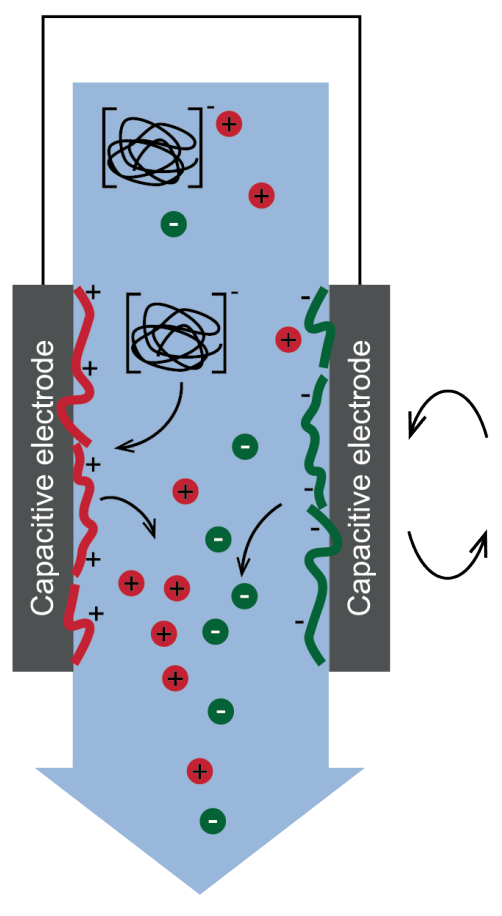

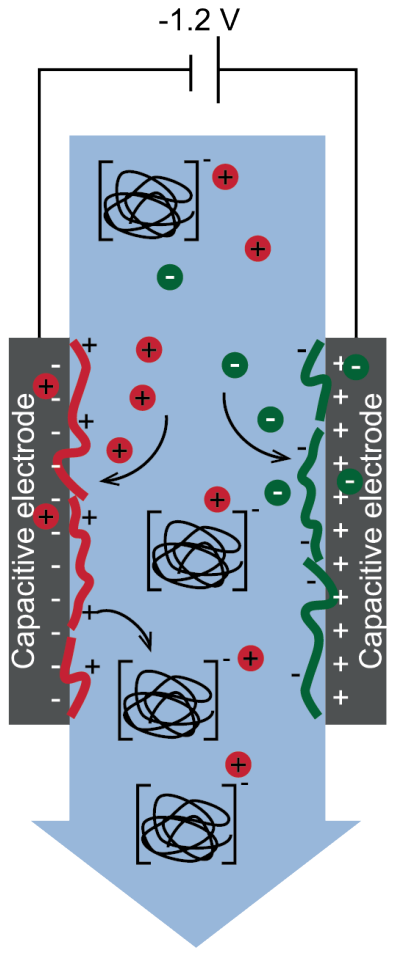

Figure 6.2. Protein and salt separation using an electrical switch. a Change in UV signal of the eluent during cycle 5. b Change in conductivity of the eluent during cycle 5. c Schematic of salt and protein adand desorption. At $0 \mathrm{~V}$ proteins adsorb and salt is released, while at a potential bias proteins desorb and salt is stored in the electrodes.

It is important to mention that protein desorption occurs faster than adsorption for all electrodes (Figure $6.3 \mathrm{c}$ ). For example, when adsorption and desorption periods 
are both set to $1800 \mathrm{~s}$ the adsorption peak of $\mathrm{Comp}^{+/-}$has a long tail and a total width of $1800 \mathrm{~s}$ (Figure $6.2 \mathrm{a}$ and $\mathbf{c}$ ), the desorption peak has however only a width of 880 $\mathrm{s}$. This asymmetry in rates is due to the interaction between the different ionic species, and the difference in driving forces during ad- and desorption. The largest contributor to this effect is the electric potential applied during the protein desorption phase, which compensates chemical charges in the electrode and which increases electrostatic repulsion of negatively charged species at the interface of the cathode while adsorbing cations. The latter effect can also be of relevance since the increase in ionic strength in the electrodes can further weaken electrostatic interactions between the proteins and the electrodes.

a

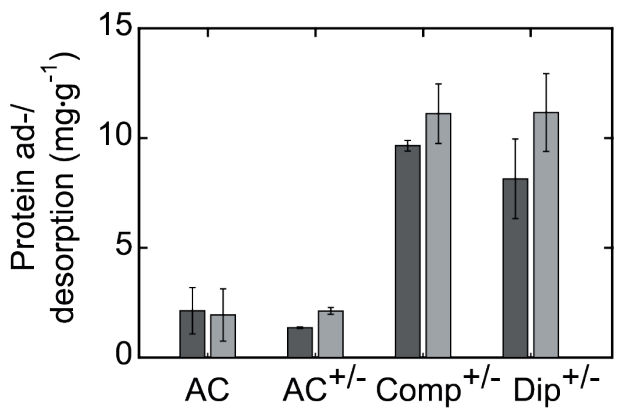

C

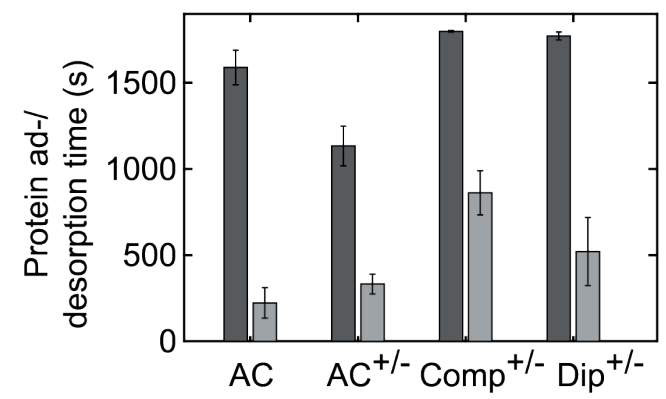

b
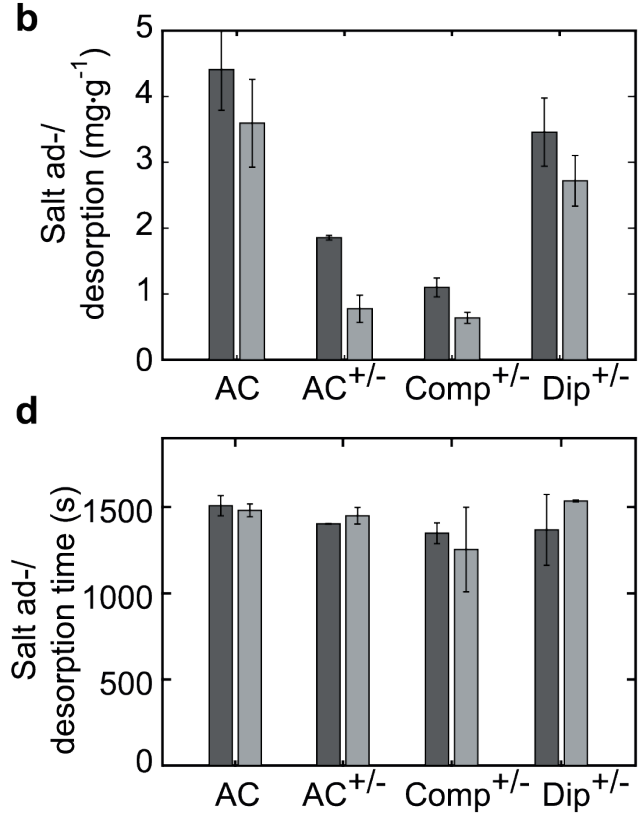

Figure 6.3. Protein and salt exchange for all electrode combinations while keeping both the adsorption and desorption cycle at $1800 \mathrm{~s}$. a Protein adsorption (dark grey) and desorption (light grey). b Salt adand desorption. $\mathbf{c}$ Width of protein ad- and desorption peaks. $\mathbf{d}$ Width of salt ad- and desorption peaks. All values in c-d are averages over cycles 3 to 10 for all replicates.

This asymmetry is also known in ion exchange processes in which the replacement of a slower diffusing molecule with a faster one is usually much faster than the opposite scenario. Ion exchange is, for example, three times faster if a cation exchange resin, loaded with $\mathrm{Na}^{+}$, is exposed to $\mathrm{H}^{+}$, than in the opposite case. This is due to the buildup of a potential gradient depleting the film around the resin if fastmoving $\mathrm{H}^{+}$ions are loaded onto the resin (Wesselingh et al., 1995). In our case, this rate determining factor could be especially important for polyelectrolyte containing 
electrodes. Since proteins diffuse much more slowly than chloride ions, protein adsorption is slow if the chemical charges in the polyelectrolyte are screened by $\mathrm{Cl}^{-}$ ions. In contrast, if the protein concentration in the polymeric layer is high, the ion concentration in its vicinity is high and thus facilitates protein- $\mathrm{Cl}^{-}$exchange.

In general, the adsorbed amounts of protein are slightly smaller than the desorbed amounts, and the same trend is found when adsorption and desorption periods are both set to either 900 or $300 \mathrm{~s}$ (Figure 6.4 and S6.11-S6.12). For these times, the amount of adsorbed protein is 5 , and $3 \mathrm{mg}^{\cdot} \mathrm{g}^{-1}$, respectively. Only when a longer adsorption period (1800 s) is combined with a shorter desorption period of $300 \mathrm{~s}$ are the amounts similar (Figure 6.4 and S6.13). Interestingly, this particular combination seems to lead to higher protein adsorption compared to the process run at $1800 \mathrm{~s}$ for adsorption and desorption, which could be indicative of slow additional adsorption to the anode between the moment when the desorption peak has leveled off, and the end of the active phase due to the applied potential.
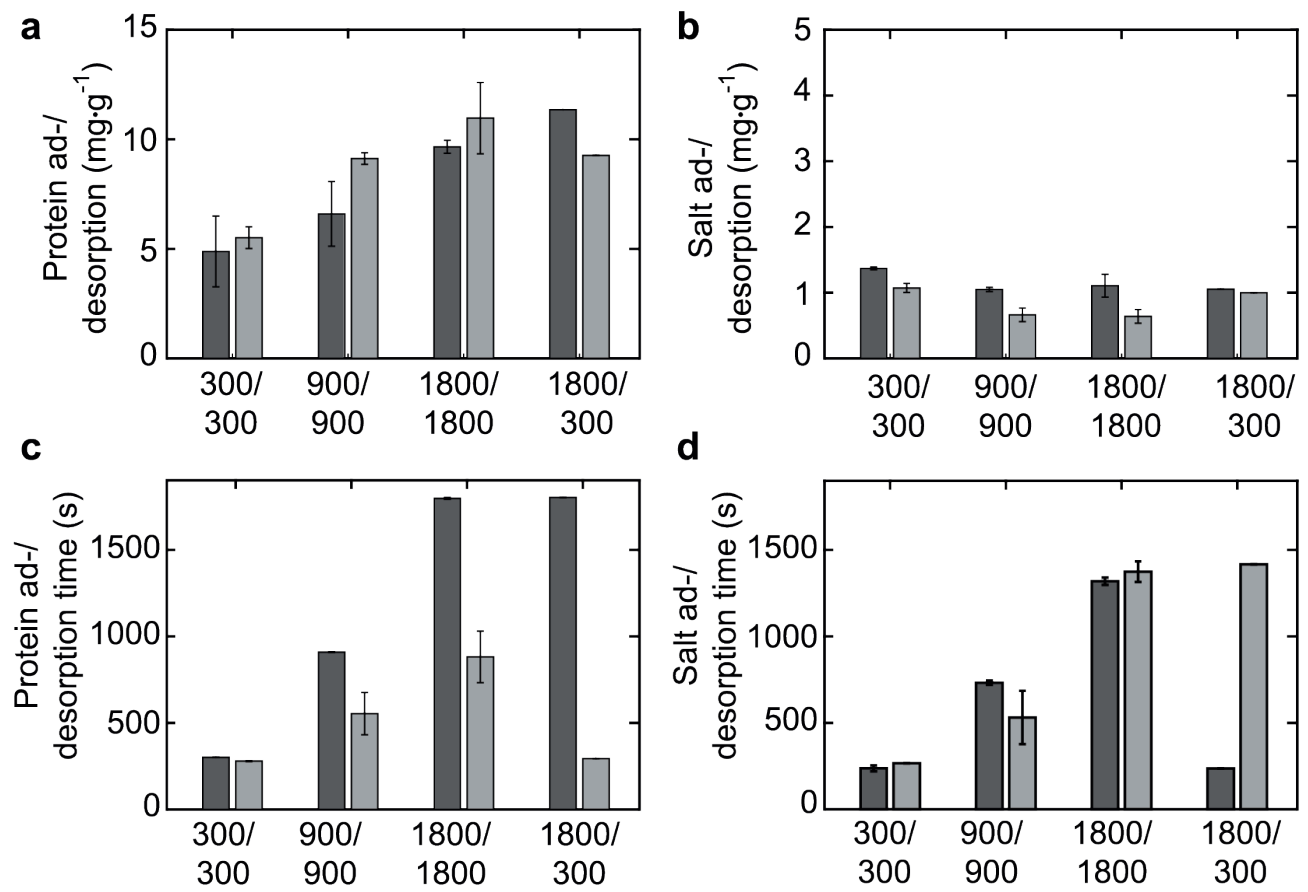

Figure 6.4. Comp $^{+/}$electrodes used at different ad- and desorption times indicated on the x-axis (adsorption time/desorption time). a Protein adsorption (dark grey) and desorption (light grey). b Same but now for salt. c Width of protein ad- and desorption peaks. $\mathbf{d}$ Same for salt.

The Comp ${ }^{+/}$electrodes contain slightly more polymer than the $\mathrm{Dip}^{+/}$electrodes (2.6 wt\% versus $1.6 \mathrm{wt} \%$, Table 6.3). This is especially relevant for the anode, since the presence of the polymer reduces protein adsorption on the 'wrong' electrode (the 
anode). If, for example, two Comp ${ }^{+}$electrodes are used (Figure S6.9), protein desorption can be detected during the active as well as the passive phase while if two Comp- electrodes are used (Figure S6.10) desorption occurs only during the active phase, with small adsorption peaks during the passive phase. The migration of proteins into the anode also occurs for $A C$ and $\mathrm{AC}^{+/-}$electrode combinations. This explains the second desorption peak right after the adsorption peak in the passive phase (Figure $6.2 \mathrm{a}$ ).

By analyzing fractions of the effluent using HPLC we were able to relate the overall WPI protein concentration to that of its three major constituents, $\beta$-lactoglobulin, $\alpha$-lactalbumin, and bovine serum albumin (Morr and $\mathrm{Ha}, 1993$ ). Of these proteins, $\beta$-lactoglobulin is the most prevalent, and its relative concentration increases during the active phase, meaning that the effluent is enriched in $\beta$-lactoglobulin (Figure 6.5).
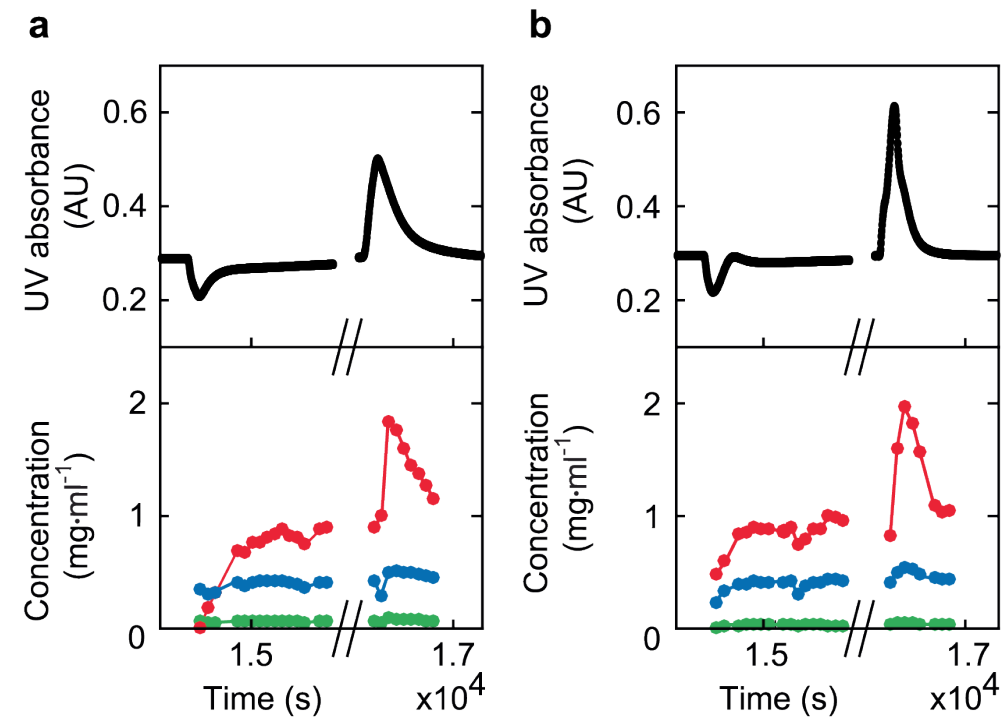

Figure 6.5. Changes in $\beta$-lactoglobulin (red), $\alpha$-lactalbumin (blue) and bovine serum albumin (green) concentration during active and passive phases (bottom) with respect to total UV absorbance change (top). a Using Comp ${ }^{+/-}$electrodes. b Using Dip ${ }^{+/-}$electrodes.

In addition to proteins, the low-molecular weight ions need to be considered. As discussed above, some ions are stored due to an ion exchange mechanism, especially when considering the electrodes containing polyelectrolytes, leading to ion adsorption when proteins are released and desorption while proteins are adsorbed. The largest driving force the ions experience is, however, the applied potential difference of $-1.2 \mathrm{~V}$, leading to ion storage in the electric double layer of the electrodes as is the case in capacitive deionization (Figure 6.2 b and $\mathbf{c}$ ). However, the amount of stored ions is 
also strongly dependent on the properties of the electrodes (Figure $6.3 \mathrm{~b}$ ). $\mathrm{AC}+$ - or Comp+/- electrode pairs that have chemical charges directly at the surface or distributed within the electrode matrix, show low salt ad- and desorption values $(0.5 \mathrm{mg} \cdot \mathrm{g}-1)$. This could be explained by less efficient capacitive ion storage, e.g. due to a negative

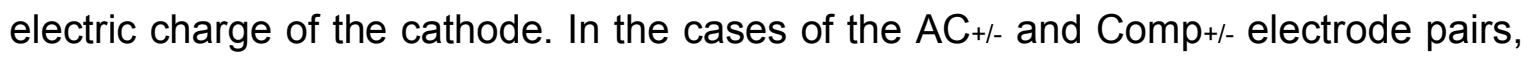
the cathode also carries positive chemical charges which partially repel the cations. This was not the case for Dip+/- electrode pairs that allow ions to penetrate through the polymer layer, thus not impacting electric double layer formation due to capacitive current. As a result, ad- and desorption is high at around $3 \mathrm{mg} \cdot \mathrm{g}-1$, which is comparable to the values obtained for AC electrodes.

The $\mathrm{Dip}^{+/-}$electrodes are also the most stable ones, with less fouling over repeated operation. Over the course of 10 cycles the gSAC of $\mathrm{Dip}^{+/-}$electrodes decreases by $0.3 \mathrm{mg} \cdot \mathrm{g}^{-1}$ while AC electrodes loos $0.7 \mathrm{mg}^{\circ} \mathrm{g}^{-1}$ (Figure S6.4 \& S6.6). This proves that the incorporation of polymers either in or on top of AC electrodes results in a capacitive protein separation process that also affects the salt concentration in the effluent depending on the position of the polymers. The Dip ${ }^{+/}$ electrode pair is most efficient per amount of polymer used, probably due to the density of the polymeric layers.

\subsection{Conclusion}

We have presented a novel capacitive, selective protein separation process based on electrically switched ion exchange. As it is an exchange process, the protein and salt ad- and desorption are coupled. Via surface modification with charged polymers we increase the protein ad- and desorption to $10 \mathrm{mg}^{\circ} \mathrm{g}^{-1}$, from $1-2 \mathrm{mg}^{\cdot} \mathrm{g}^{-1}$ for the unmodified electrodes, and even achieved an enrichment of $\beta$-lactoglobulin over other whey proteins. The stability of the modified electrodes remained high. Since protein and salt ad- and desorbed in opposite half cycles this process is promising for concentration and desalting of protein streams within one process, which is relevant to industries such as food and biotechnology. 


\subsection{Supporting Information}
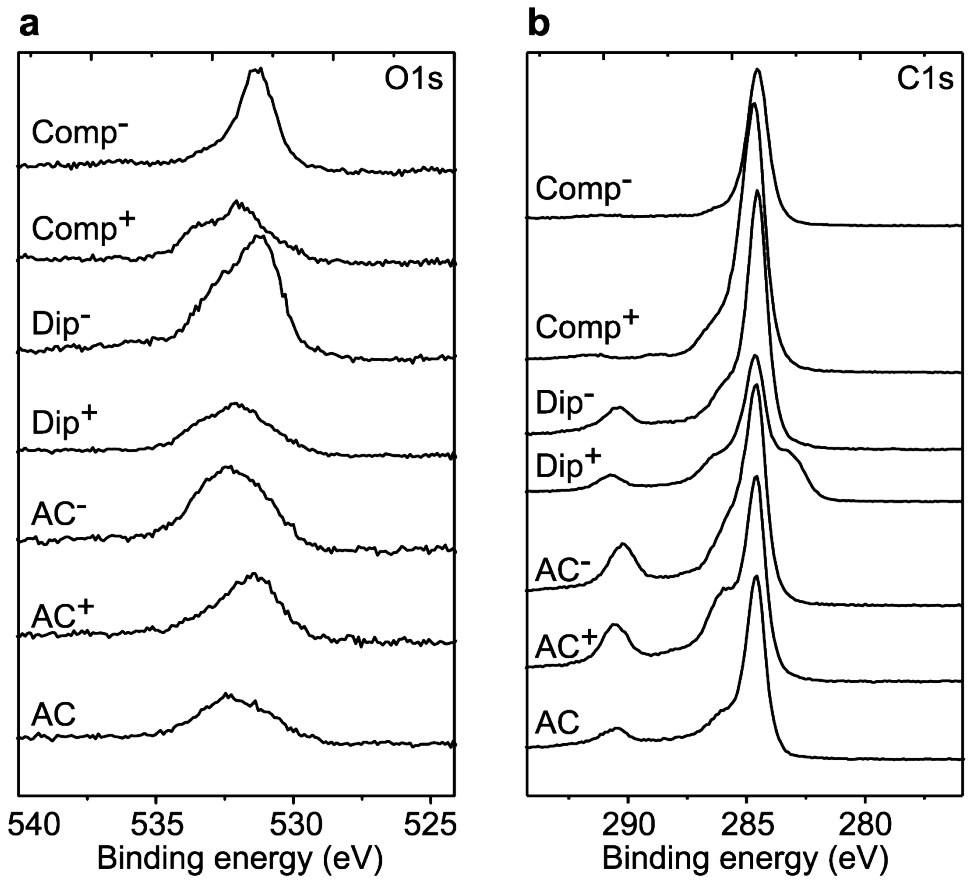

Figure S6.1. X-ray photoelectron spectroscopy narrow scans of a O1s peak and $\mathbf{b}$ C1s peak.

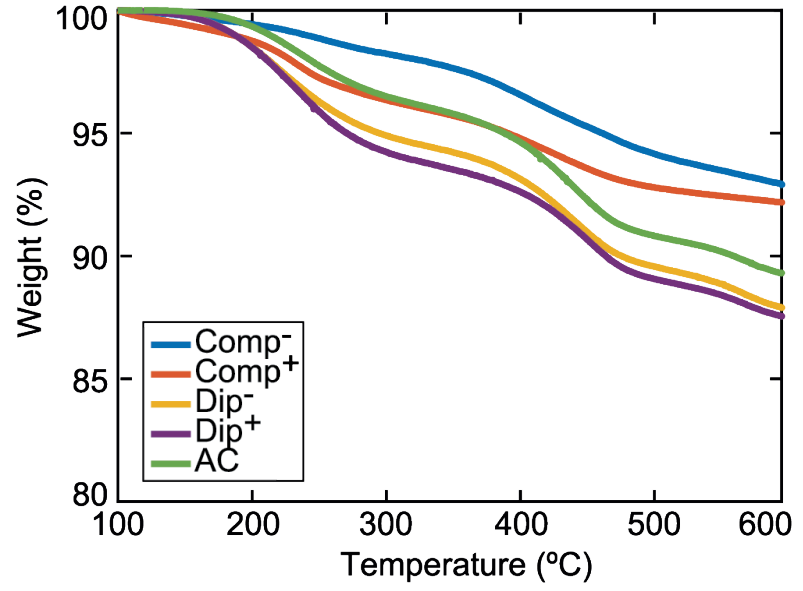

Figure S6.2. Thermogravimetric weight loss after heating samples from 100 to $600{ }^{\circ} \mathrm{C}$ (Note: The curves of $\mathrm{AC}^{-}$and $\mathrm{AC}^{+}$are not shown since they overlapped with $\mathrm{AC}$ ). 

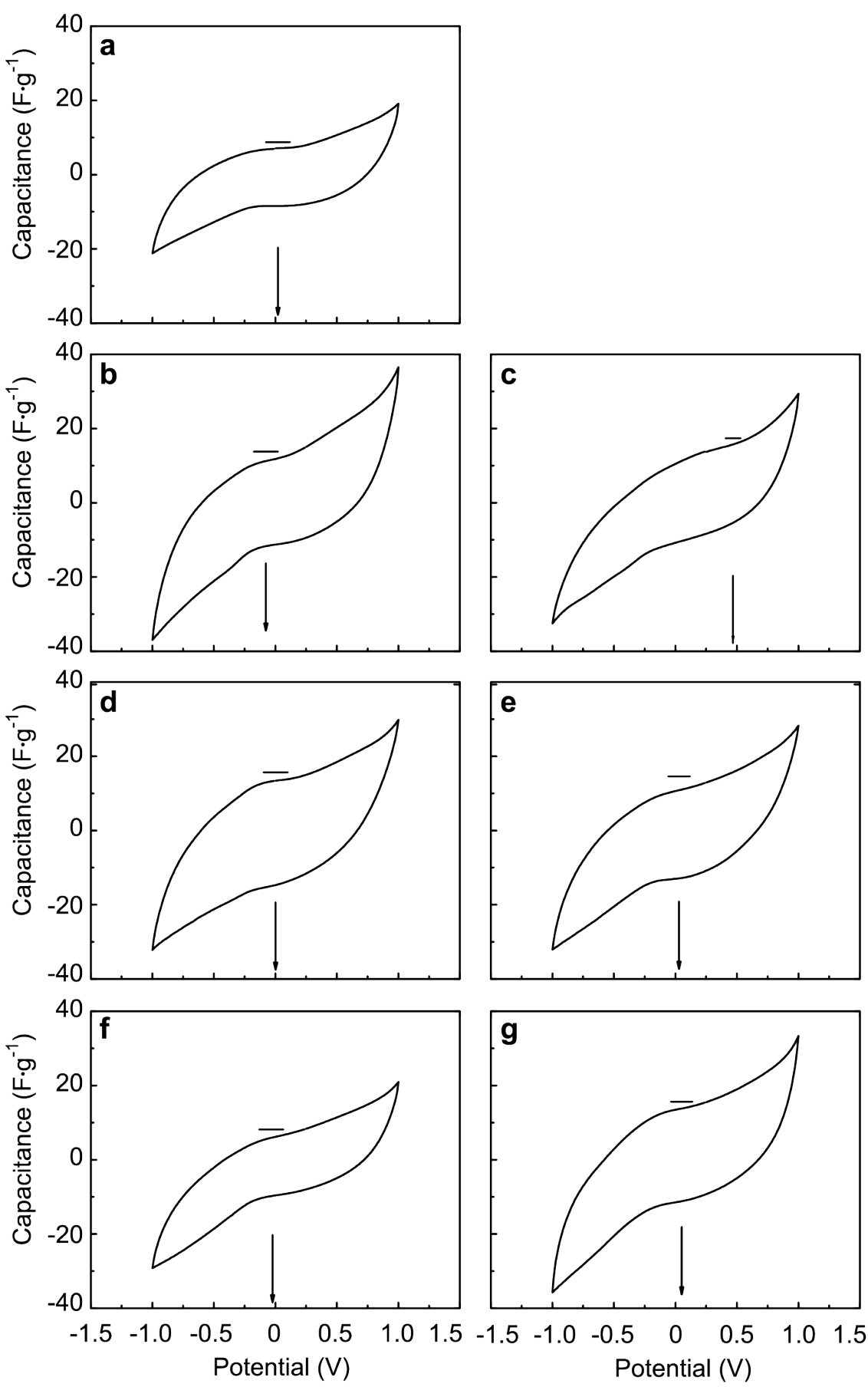

Figure S6.3. CV scans of a AC electrode b $\mathrm{AC}^{+} \mathbf{c} \mathrm{AC}^{-} \mathbf{d} \mathrm{Comp}^{+}$e Comp f $^{-} \mathrm{Dip}^{+} \mathbf{g}$ Dip-. The arrow indicates the position of the potential of zero charge. 

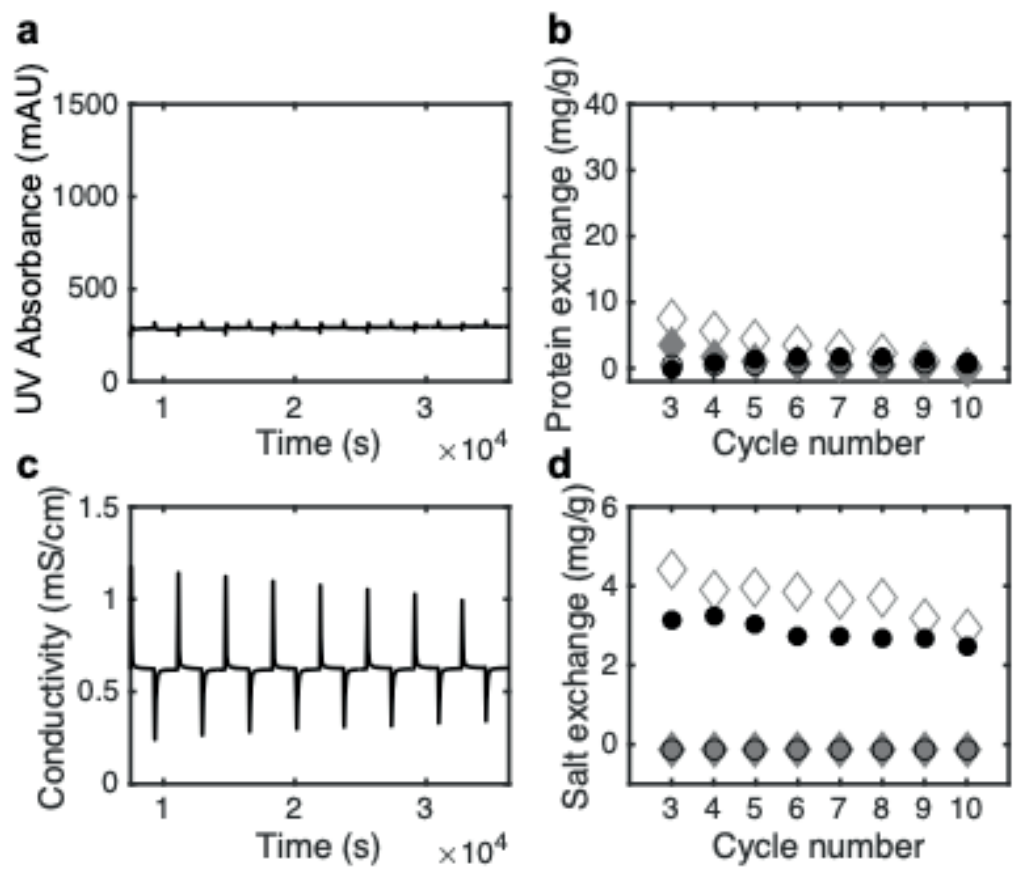

Figure S6.4. UV absorbance (a) and conductivity signal (c) measured in the outlet of the electrochemical flow cell when using $A C$ electrodes, using an adsorption and desorption time of $1800 \mathrm{~s}$ and a potential of $-1.2 \mathrm{~V}$ during the active phase. $\mathbf{b}$ and $\mathbf{d}$ represent the amount of proteins and salt exchanged during the passive and active phase, respectively. The grey filled diamond indicates adsorption in the passive phase and the empty diamond indicates adsorption in the active phase, while the black filled circle indicates desorption in the passive phase and the empty circle indicates desorption in the active phase.
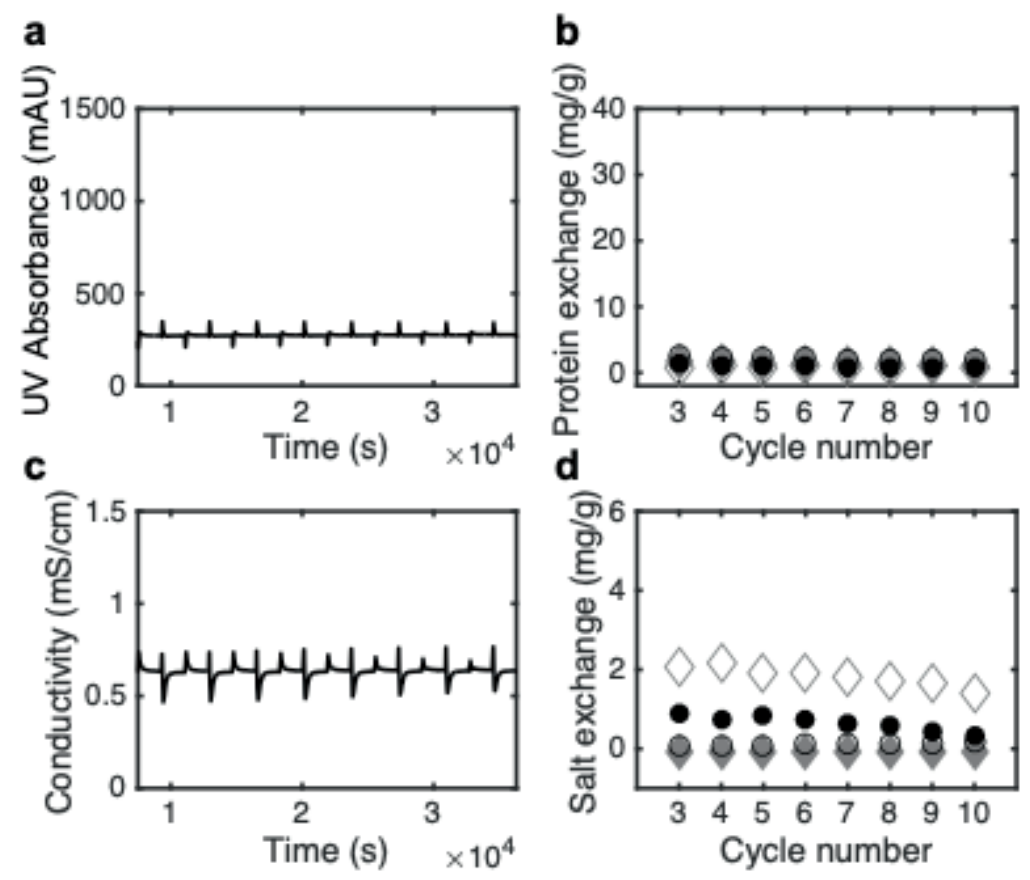

Figure S6.5. UV absorbance (a) and conductivity signal (c) measured in the outlet of the electrochemical flow cell when using $\mathrm{AC}^{+/-}$electrodes, an adsorption and desorption time of $1800 \mathrm{~s}$ and a potential of $-1.2 \mathrm{~V}$ during the active phase. $\mathbf{b}$ and $\mathbf{d}$ represent the amount of proteins and salt exchanged during the passive and active phase respectively. The grey filled diamond indicates adsorption in the passive phase and the empty diamond indicates adsorption in the active phase, while the black filled circle indicates desorption in the passive phase and the empty circle indicates desorption in the active phase. 

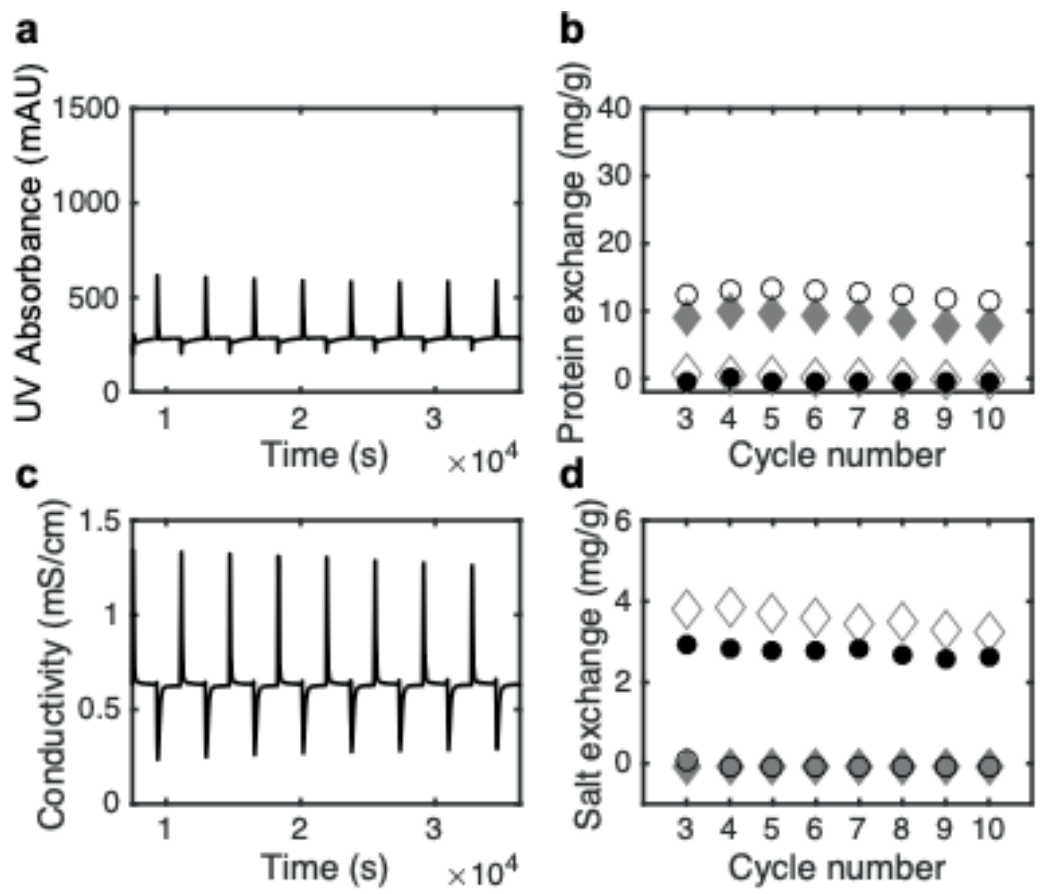

Figure S6.6. UV absorbance (a) and conductivity signal (c) measured in the outlet of the electrochemical flow cell when using $\mathrm{Dip}^{+/-}$electrodes, an adsorption and desorption time of $1800 \mathrm{~s}$ and a potential of $-1.2 \mathrm{~V}$ during the active phase. $\mathbf{b}$ and $\mathbf{d}$ represent the amount of proteins and salt exchanged during the passive and active phase respectively. The grey filled diamond indicates adsorption in the passive phase and the empty diamond indicates adsorption in the active phase, while the black filled circle indicates desorption in the passive phase and the empty circle indicates desorption in the active phase.
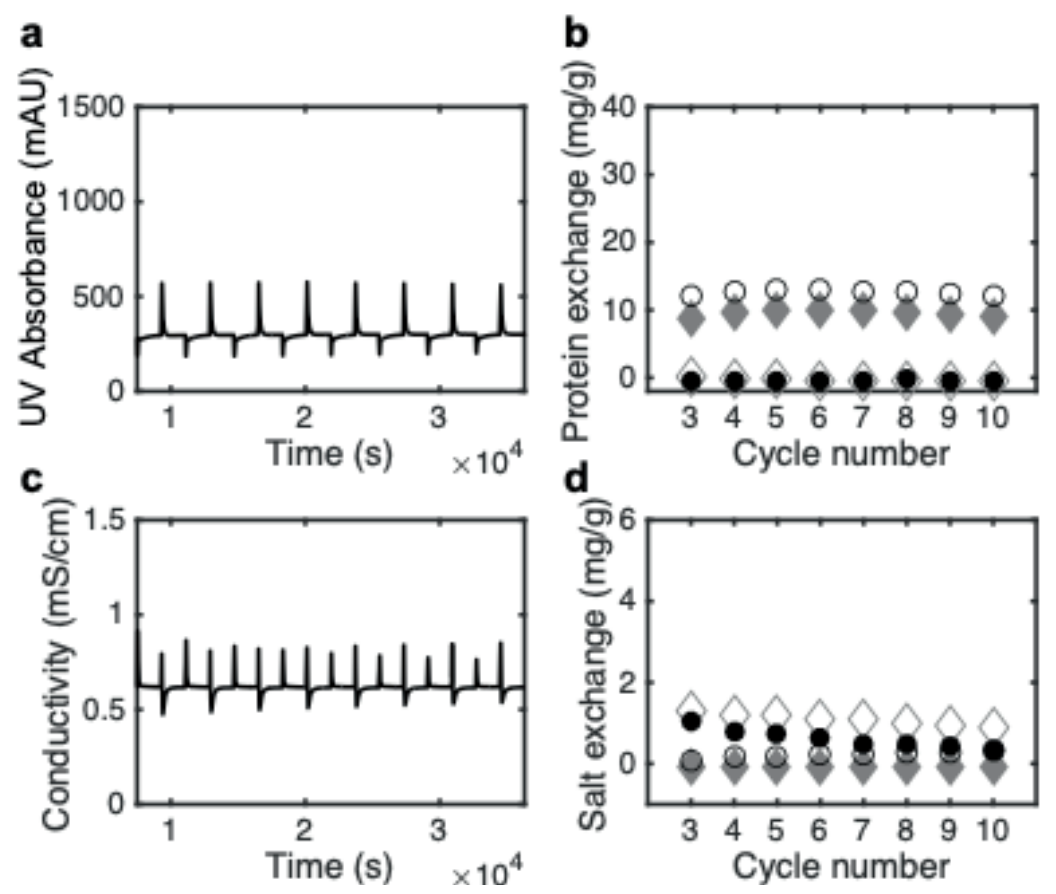

Figure S6.7. UV absorbance (a) and conductivity signal (c) measured in the outlet of the electrochemical flow cell when using Comp ${ }^{+/-}$electrodes, an adsorption and desorption time of $1800 \mathrm{~s}$ and a potential of $-1.2 \mathrm{~V}$ during the active phase. $\mathbf{b}$ and $\mathbf{d}$ represent the amount of proteins and salt exchanged during the passive and active phase respectively. The grey filled diamond indicates adsorption in the passive phase and the empty diamond indicates adsorption in the active phase, while the black filled circle indicates desorption in the passive phase and the empty circle indicates desorption in the active phase. 

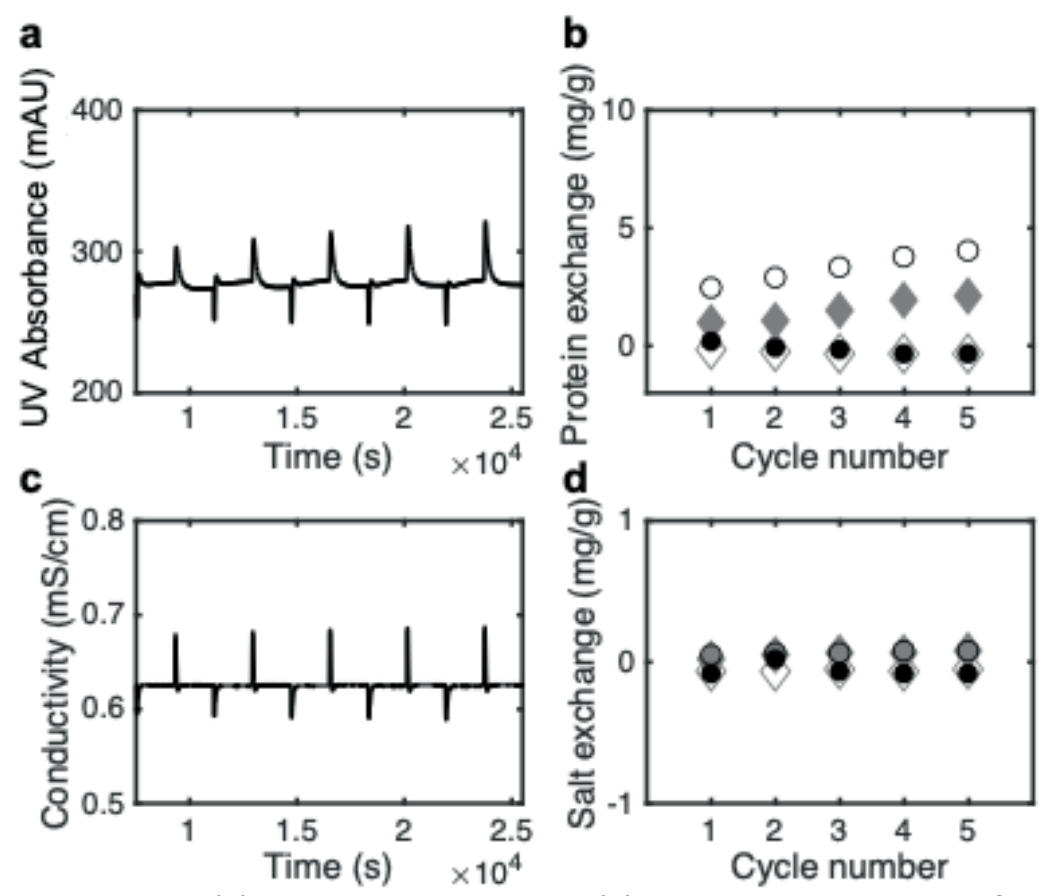

Figure S6.8. UV absorbance (a) and conductivity signal (c) measured in the outlet of the electrochemical flow cell when using Comp ${ }^{+-}$electrodes, an adsorption and desorption time of $1800 \mathrm{~s}$ and a potential of $-0.4 \mathrm{~V}$ during the active phase. $\mathbf{b}$ and $\mathbf{d}$ represent the amount of proteins and salt exchanged during the passive and active phase respectively. The grey filled diamond indicates adsorption in the passive phase and the empty diamond indicates adsorption in the active phase, while the black filled circle indicates desorption in the passive phase and the empty circle indicates desorption in the active phase.
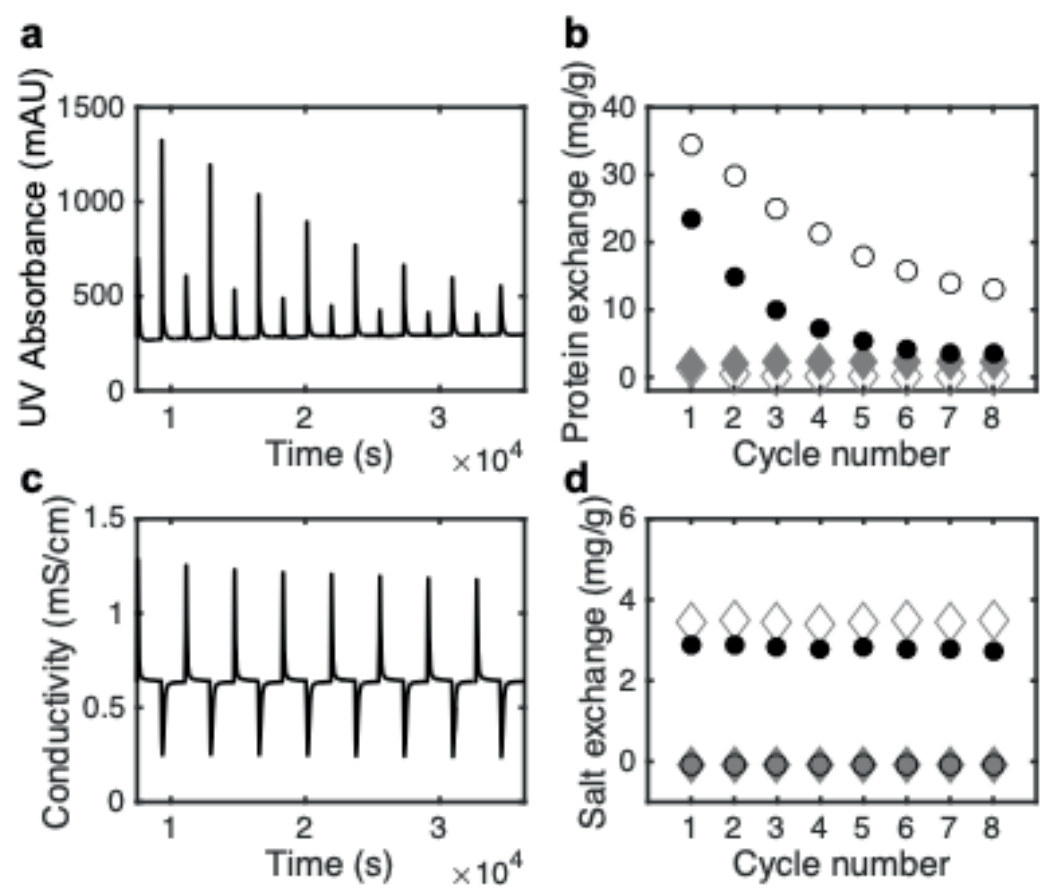

Figure S6.9. UV absorbance (a) and conductivity signal (c) measured in the outlet of the electrochemical flow cell when using two Comp ${ }^{+}$electrodes, an adsorption and desorption time of $1800 \mathrm{~s}$ and a potential of $-1.2 \mathrm{~V}$ during the active phase. $\mathbf{b}$ and $\mathbf{d}$ represent the amount of proteins and salt exchanged during the passive and active phase respectively. The grey filled diamond indicates adsorption in the passive phase and the empty diamond indicates adsorption in the active phase, while the black filled circle indicates desorption in the passive phase and the empty circle indicates desorption in the active phase. 

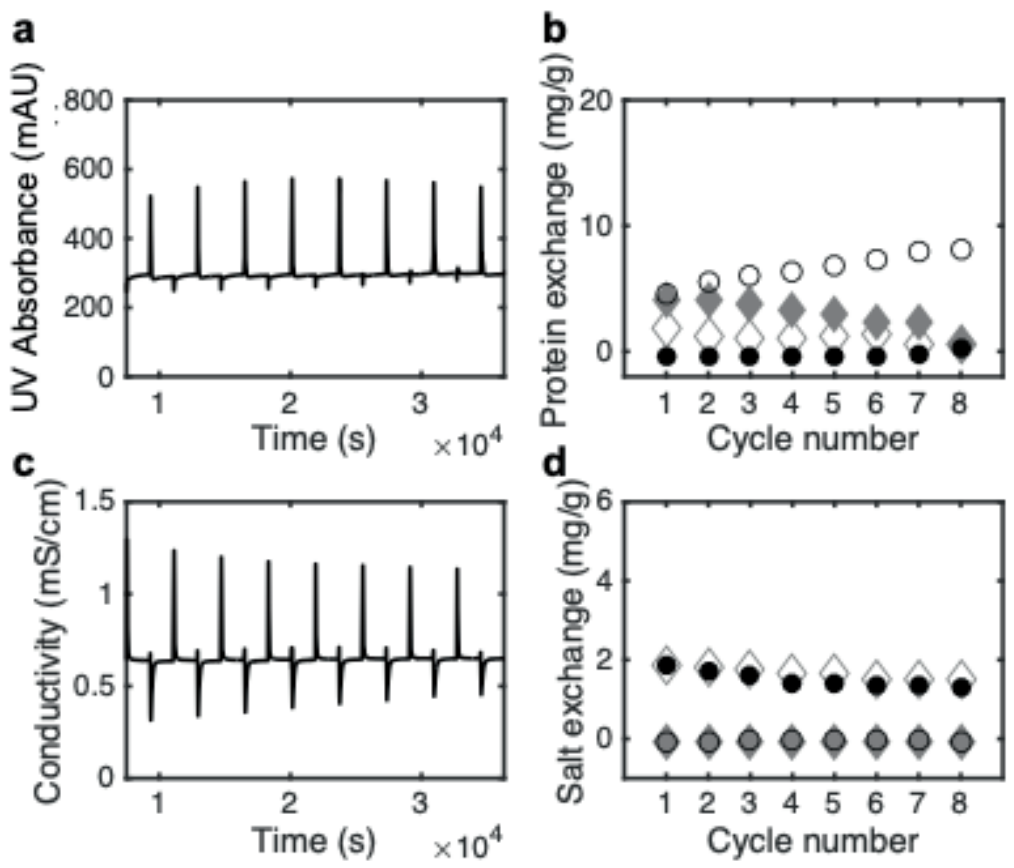

Figure 56.10. UV absorbance (a) and conductivity signal (c) measured in the outlet of the electrochemical flow cell when two Comp- electrodes, an adsorption and desorption time of $1800 \mathrm{~s}$ and a potential of $-1.2 \mathrm{~V}$ during the active phase. $\mathbf{b}$ and $\mathbf{d}$ represent the amount of proteins and salt exchanged during the passive and active phase respectively. The grey filled diamond indicates adsorption in the passive phase and the empty diamond indicates adsorption in the active phase, while the black filled circle indicates desorption in the passive phase and the empty circle indicates desorption in the active phase.
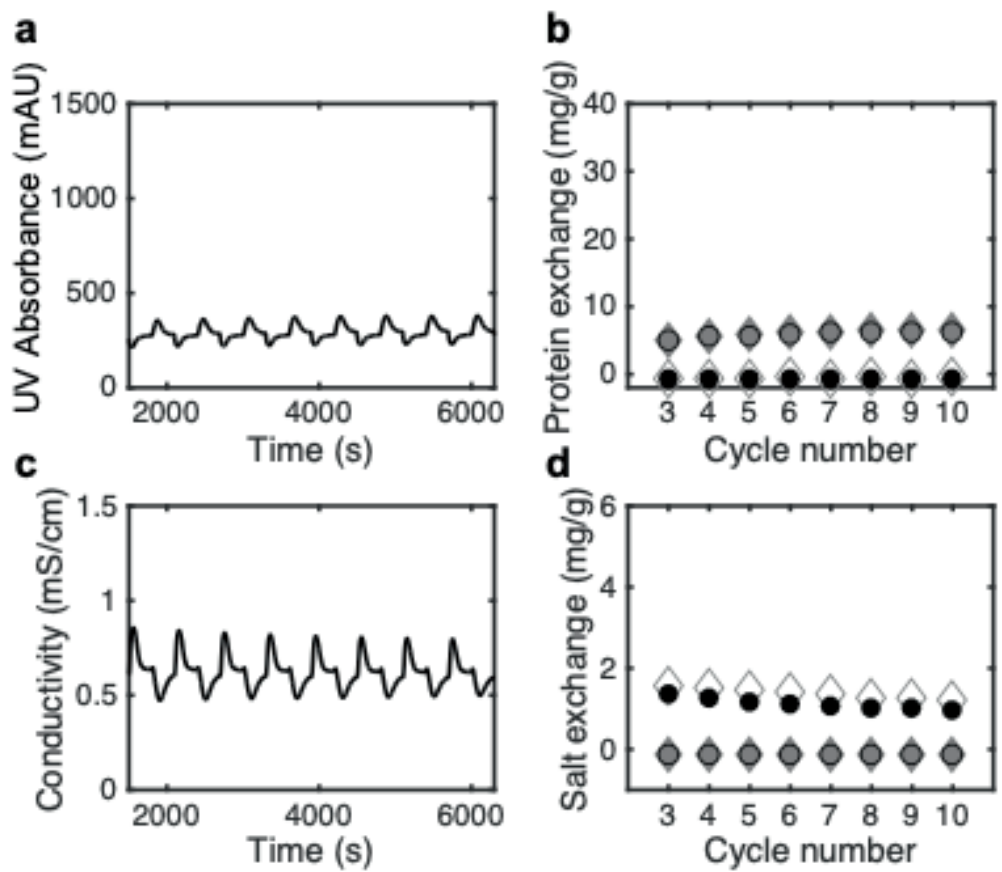

Figure S6.11. Indicators MAC/PSS matrix AD: UV absorbance (a) and conductivity signal (c) measured in the outlet of the electrochemical flow cell when using $\mathrm{Comp}^{+/-}$electrodes, at ad- and desorption time of $300 \mathrm{~s}$, and a potential of $-1.2 \mathrm{~V}$ during the active phase. $\mathbf{b}$ and $\mathbf{d}$ represent the amount of proteins and salt exchanged during the passive and active phase respectively. The grey filled diamond indicates adsorption in the passive phase and the empty diamond indicates adsorption in the active phase, while the black filled circle indicates desorption in the passive phase and the empty circle indicates desorption in the active phase. 

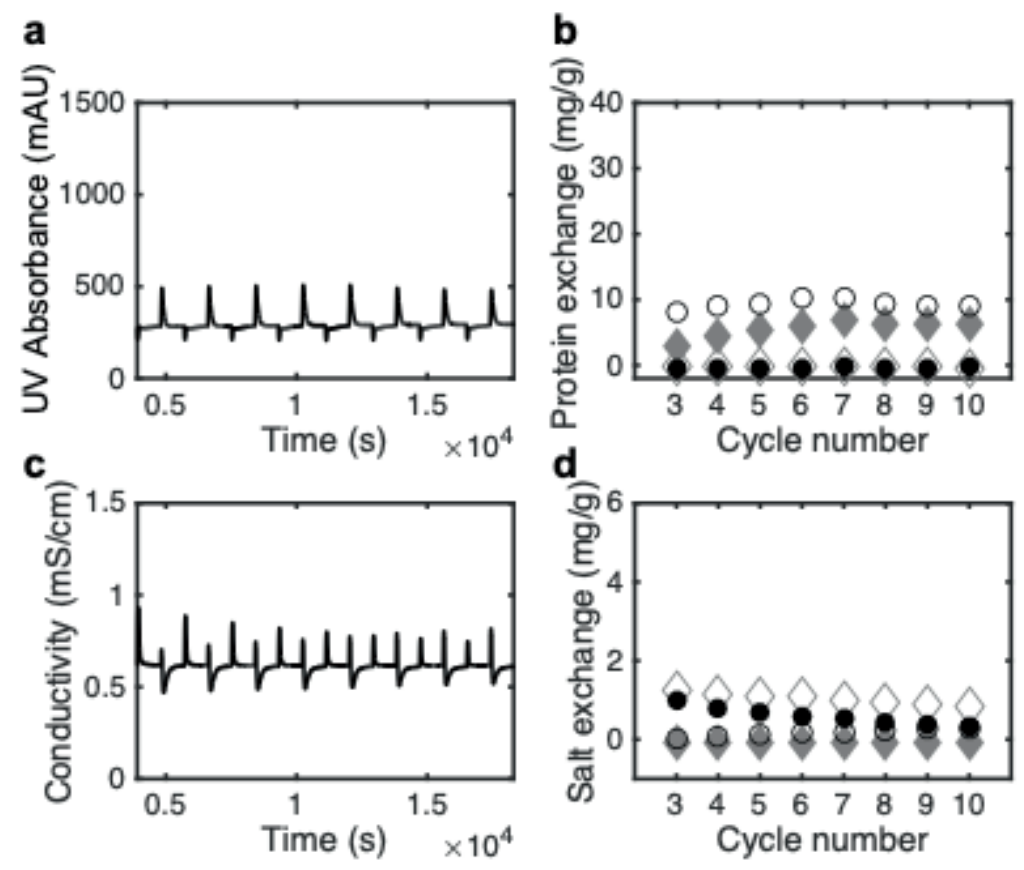

Figure S6.12. Indicators MAC/PSS matrix AD: UV absorbance (a) and conductivity signal (c) measured in the outlet of the electrochemical flow cell when using $\mathrm{Comp}^{+/}$electrodes, at ad- and desorption times of $900 \mathrm{~s}$, and a potential of $-1.2 \mathrm{~V}$ during the active phase. b) and d) represent the amount of proteins and salt exchanged during the passive and active phase respectively. The grey filled diamond indicates adsorption in the passive phase and the empty diamond indicates adsorption in the active phase, while the black filled circle indicates desorption in the passive phase and the empty circle indicates desorption in the active phase.
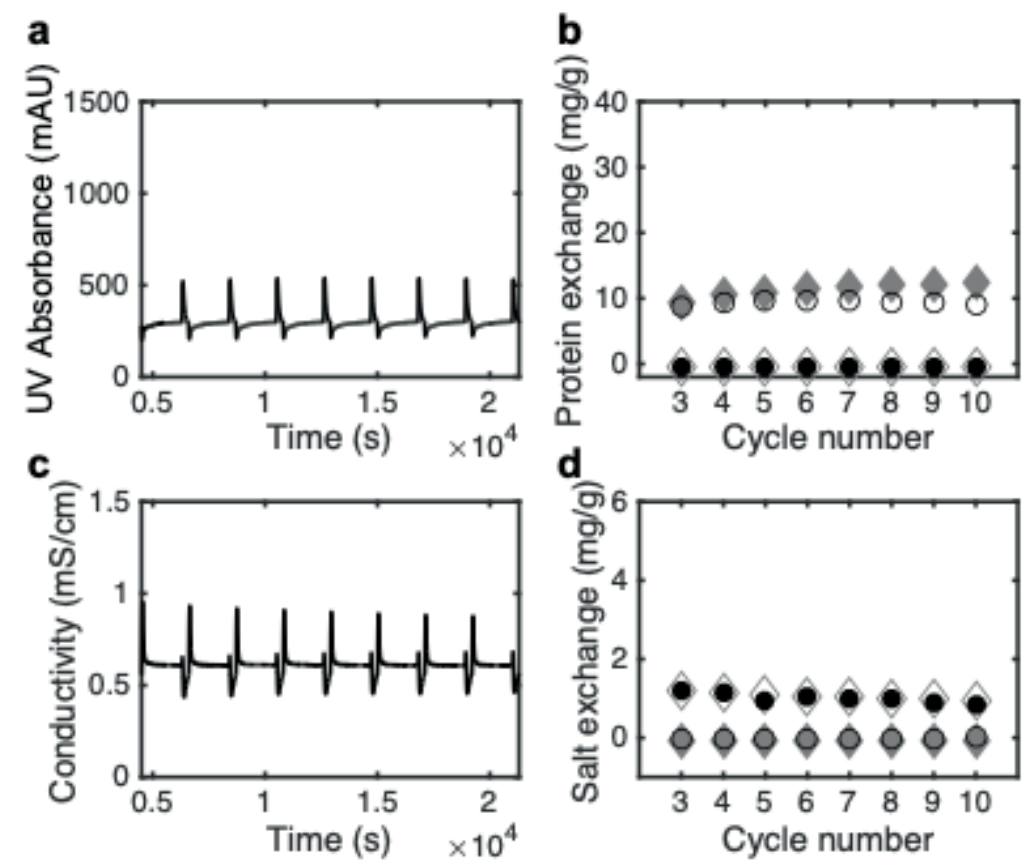

Figure S6.13. Indicators MAC/PSS matrix AD: UV absorbance (a) and conductivity signal (c) measured in the outlet of the electrochemical flow cell when using Comp ${ }^{+/-}$electrodes, at ad- and desorption time of $1800 \mathrm{~s}$ and $300 \mathrm{~s}$, respectovely, at a potential of $-1.2 \mathrm{~V}$ during the active phase. $\mathbf{b}$ and $\mathbf{d}$ represent the amount of proteins and salt exchanged during the passive and active phase respectively. The grey filled diamond indicates adsorption in the passive phase and the empty diamond indicates adsorption in the active phase, while the black filled circle indicates desorption in the passive phase and the empty circle indicates desorption in the active phase. 



\section{CHAPTER 7}

\section{CONTROLLING ELECTROSTATIC AND HYDRATION INTERACTIONS ELECTROCHEMICALLY FOR PROTEIN ADSORPTION AND DESORPTION}

When proteins in aqueous solution are exposed to solid substrates, they adsorb due to the dynamic interplay of electrostatic, van der Waals and hydration interactions. Once adsorbed it is very challenging to induce desorption. Here we use a gold electrode as the solid substrate and modulate the surface potential to systematically investigate adsorption as well as desorption, the latter being an essential but troublesome step in protein recovery. We show through surface plasmon resonance, atomic force microscopy, and electrowetting that biasing the electrode to more negative potentials (by $-0.4 \mathrm{~V}$ compared to the open circuit potential at $\mathrm{pH} 6$ ) results for the negatively charged protein $\beta$-lactoglobulin in an increase of the adsorption barrier by $6 \mathrm{~kJ} \cdot \mathrm{mol}^{-1}$. We clearly demonstrate that this applied potential results in an increased double layer potential of $-0.06 \mathrm{~V}$ and an increase in hydration repulsion. This indicates that an electric potential can directly influence the adsorption free energy, and it is even possible to use it to induce $\beta$-lactoglobulin desorption. These principles can be used in innovative separation technology that uses only one trigger to steer protein ad- and desorption.

This chapter has been submitted as:

P. A. Fritz, B. Bera, J. van den Berg, I. Visser, M. Kleijn, R. M. Boom, and K. Schroën. Controlling electrostatic and hydration interactions electrochemically for protein adsorption and desorption. 


\subsection{Introduction}

Although protein adsorption to and desorption from solid interfaces is of great importance in many application fields such as biotechnology, biophysics, medicine, pharmaceutics, and food science, the underlying mechanisms are far from resolved, due to their complexity. For this, multiple components (e.g. surface, protein, solvent), that have different properties (e.g. polarity, charge) depending on the environment they are exposed to $(\mathrm{pH}$, isoelectric strength, temperature) and thus contribute in various ways to system specific interactions would need to be considered. Further, these effects occur dynamically: when a solid surface is brought into contact with a protein solution, the surface will first be wetted by solvent molecules. Subsequently, proteins diffuse into the near-surface fluid, replace solvent molecules and adsorb, leading to an adsorbed layer where conformational rearrangements and replacements can still occur (Nakanaishi et al., 2001; Norde, 1995; Rabe et al., 2011).

Since proteins can have multiple contact points with the surface, the Gibbs free energy of adsorption is usually in the range of tens of $R T$ per mole of protein, with $R$ the universal gas constant and $T$ the temperature (Norde, 1995; Vogler, 2012). For $\beta$-lactoglobulin values between $-5 \mathrm{~kJ} \cdot \mathrm{mol}^{-1}(-2 R T)$ to $-55 \mathrm{~kJ} \cdot \mathrm{mol}^{-1}(-22 R T)$ depending on the surface (Bonomo et al., 2006; Fainerman et al., 2006; Omanovic and Roscoe, 2000), have been reported; the negative sign denotes attraction between proteins and surface (Norde, 1995; Vogler, 2012). Electrostatic interactions as well as van der Waals attraction, and hydration interaction are key factors defining the origin and strength of the binding of proteins to surfaces (Israelachvili, 2011; Kleijn et al., 2004; Lyklema, 2000; Mcumber et al., 2015). Once proteins adsorb, desorption is challenging due to structural rearrangements and relaxation at the interface (Norde, 1995). E.g. for $\beta$-lactoglobulin, maximum desorption upon rinsing with buffer could be achieved after around $1500 \mathrm{~s}$ of adsorption (the critical surface coverage at a concentration of $5 \cdot 10^{-8} \mathrm{M}$ ); for shorter and longer adsorption times, the amount of protein that could be removed was less (Rabe et al., 2007).

Although elution with buffer is widely used to achieve protein desorption, in many cases this cannot be effective because of the high adsorption energy; in order to influence that, we hypothesize, that electrochemical stimuli are more efficient, since they directly affect the solid surface potential, and thus the binding strength by both 
electrostatic and hydration repulsion. To quantify these effects, AFM has been used to study surface interactions and characterize the electric double layer (Butt, 2007; Butt et al., 2005; Ricci et al., 2014); on a gold electrode the double layer potential could be shifted between $-30 \mathrm{mV}$ and $20 \mathrm{mV}$ when varying the applied potential form -0.3 to $0.5 \mathrm{~V}$ versus a $\mathrm{Ag} / \mathrm{AgCl}(3 \mathrm{M})$ reference electrode (Barten et al., 2003a; Hillier et al., 1996).

Here we investigate for the first time to what extent the applied potential influences protein adsorption (Barten et al., 2003b; Kleijn et al., 2004; Moulton et al., 2003), and desorption when using electrodes directly, so without electrochemically responsive surface coatings (Mu et al., 2007; Su et al., 2017a). The approach we take revolves around a unique combination of atomic force microscopy, electrowetting (Mugele and Baret, 2005) and surface plasmon resonance, which allows us to systematically investigate and quantify the fundamental effect of the surface potential on protein ad- and desorption, and the underlying interactions. The obtained findings are directly linked to concrete applications in biosensors and separation (Maharjan et al., 2008) (e.g. electrochemical separation (Su and Hatton, 2017b; Yoon et al., 2019) and electrochemically modulated liquid chromatography (Collins and Arrigan, 2009; Harnisch and Porter, 2001; Porter and Takano, 2000; Su and Hatton, 2017a; Yamini et al., 2014)).

\subsection{Materials and Methods}

\subsection{1. $\beta$-Lactoglobulin purification and characterization}

$\beta$-lactoglobulin $\left(M_{w} 18.4 \mathrm{kDa}\right)$ was purified from whey protein containing $72.4 \%$ protein following Mailliart and Ribadeau-Dumas (1988). Whey protein solution was adjusted to $\mathrm{pH} 2$ at $14 \%$ sodium chloride concentration; after overnight stirring the solution was diluted 2 -fold and centrifuged (10 $\mathrm{min}$ at $10,000 \mathrm{~g}$ ). The sodium chloride concentration of the supernatant was subsequently adjusted to $23 \%$ and again centrifuged. The pellet was resuspended and the $\mathrm{pH}$ was adjusted to 7 , and resulting $\beta$-lactoglobulin solution was dialyzed against water and freeze dried for storage. The purity of $\beta$-lactoglobulin in the final product was measured using HPLC and was at least 99.9 \% (Figure S7.1 a). The isoelectric point was 4.6 (Zetasizer measurements in the range of $\mathrm{pH} 3$ to $\mathrm{pH} 8$; Figure $\mathbf{S 7 . 1} \mathbf{b}$ ). 


\subsubsection{Surface plasmon resonance (SPR)}

A SPR-device from Kinetic Evaluation Instruments (The Netherlands) was used in combination with an electrochemical cell (Figure 7.1 a). For the baseline, $5 \mathrm{mM}$ sodium chloride solution at $\mathrm{pH} 6$ was run through the cuvette and the SPR signal recorded. Subsequently, a $10 \mathrm{mg} / \mathrm{L} \beta$-lactoglobulin solution at $5 \mathrm{mM}$ sodium chloride $(\mathrm{pH}$ 6) was introduced in the cuvette. As blank, the same experiment was conducted without protein; these values were subtracted from those obtained for the $\beta$-lactoglobulin solution to identify the signal related to protein only.

For electrochemical measurements the gold surface of the SPR chip (SSENS, The Netherlands) was the working electrode, a platinum wire the reference electrode, and stainless steel the counter electrode. The reference electrode was calibrated using an $\mathrm{Ag} / \mathrm{AgCl}(3 \mathrm{M})$ reference electrode at $0.3 \mathrm{~V} \mathrm{vs} \mathrm{Ag} / \mathrm{AgCl}$ at $\mathrm{pH}$ 6. The open circuit potential $(\mathrm{OCP})$ of the gold electrode at $\mathrm{pH} 6$ and $5 \mathrm{mM} \mathrm{NaCl}$ was $0.1 \mathrm{~V}$ vs $\mathrm{Ag} / \mathrm{AgCl}$ $(3 \mathrm{M})$.

Adsorption (typically 3 hours) was measured for different constant applied potentials to the gold electrode, or for alternating potentials. Typically, OCP would then be applied for 10 to $1800 \mathrm{~s}$, followed by $-0.4 \mathrm{~V}$ for $1800 \mathrm{~s}$, which was repeated four times. All experiments where conducted in independent triplicates. Increase in energy barrier for adsorption $(\Delta E)$ upon applying $-0.4 \mathrm{~V}$ was determined using Equation 7.1 (Norde, 1995; Norde et al., 1991),

$$
\frac{j_{-0.4 V}}{j_{O C P}}=e^{-\Delta E / R T}
$$

where $j_{-0.4 V}$ and jocp represent the initial protein adsorption rate at an applied potential of $-0.4 \mathrm{~V}$ vs OCP, and at the OCP, respectively.

\subsubsection{Atomic force spectroscopy}

A Bruker Nanoscope Multimode SPM was used in combination with an electrochemical fluid cell (Figure 7.1 b). To obtain a truncated conical blunted tip, a pointed silicon AFM probe (MikroMasch, NSC36) was scratched on a silicon oxide surface for $1 \mathrm{~h}$, using the AFM in contact mode at a deflection set point of $8 \mathrm{~V}$ (Figure 7.1 c-e) (Ebeling et al., 2011). After scratching the probe radius $R_{p}$ was $70 \mathrm{~nm}$. 
The cantilever spring constant was found to be around $0.6 \mathrm{~N} \cdot \mathrm{m}^{-1}$ before and after each measurement.

a

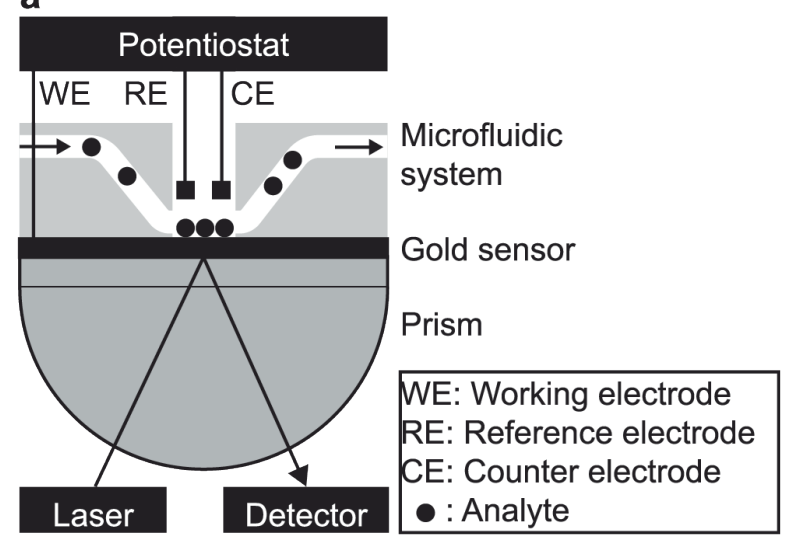

b

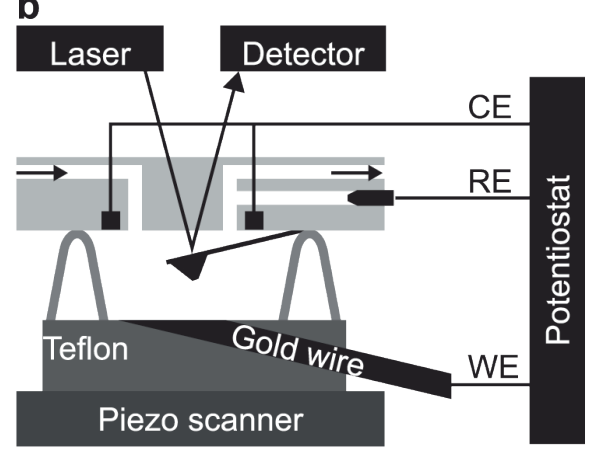

f

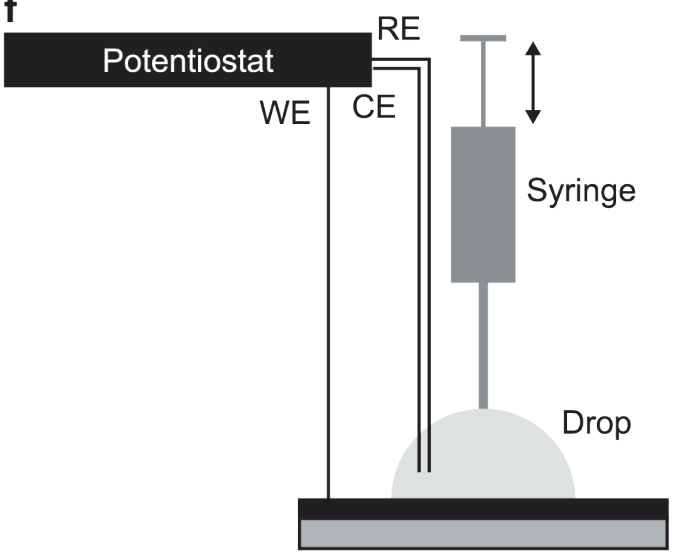

Gold sensor

C

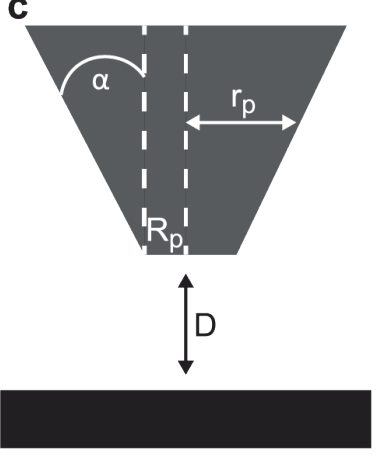

d

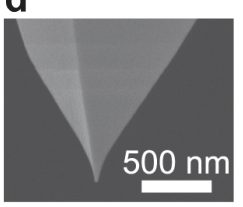

e

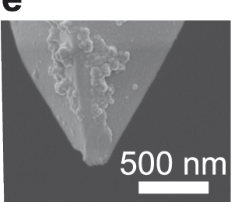

Figure 7.1 Overview of experimental set-ups, and details of probes. a Electrochemical surface plasmon resonance. b Electrochemical atomic force spectroscopy. c Truncated cone shaped probe used for atomic force spectroscopy with angle $\alpha$ and radius $R_{p}$ at the plane facing the gold substrate. $d$, e SEM image of the pointed and blunted atomic force spectroscopy probe, respectively. $\mathbf{f}$ Electrowetting.

Gold electrodes were obtained by fixing a gold wire in a Teflon sample holder and polishing the cross section $\left(2 \mathrm{~mm}^{2}\right)$ using a $40 \mathrm{~nm}$ silica suspension in water (OPU suspension, Struers bv, Denmark). This resulted in relatively flat gold surfaces containing areas with a peak-to-valley distance of less than $5 \mathrm{~nm}$ over an area of $0.1 \mu \mathrm{m}^{2}$, as determined by AFM imaging in the contact mode with a standard nitride tip (Barten et al., 2003a). The cross section of the gold wire was used as working electrode, while a platinum wire was the counter electrode, and a $3 \mathrm{M} \mathrm{Ag} / \mathrm{AgCl}$ electrode the reference electrode (Figure 7.1 b). As electrolyte, a $5 \mathrm{mM}$ sodium chloride solution at $\mathrm{pH} 6$ was used. All solutions were degassed by sonication for $30 \mathrm{~min}$ prior to the experiment. With a syringe, $10 \mathrm{~mL}$ of solution was flushed through the AFM cell, after which it was closed and the system left to equilibrate for $10 \mathrm{~min}$, 
after which force curves were measured over $500 \mathrm{~nm}$ at a ramp rate of $1 \mathrm{~Hz}$, biasing the working electrode to $0,-0.1,-0.2,-0.3$ and $-0.4 \vee$ vs OCP, respectively.

\subsubsection{Electrowetting}

The dynamic contact angle was measured (Teclis, France) on the same gold substrate used for the SPR experiments, and used as working electrode. As counter and reference electrodes, Pt-wires were immersed into the drop $(\mathrm{NaCl} 5 \mathrm{mM})$ as indicated in Figure 7.1 f. The drop was regulated using a 16G needle (Teclis, France) at $1 \mathrm{~mm}^{3} \cdot \mathrm{s}^{-1}$ and advancing and receding contact angles on the left and right side of the drop were measured. After a period of $50 \mathrm{~s}$, a potential ramp from 0 to $-0.5 \mathrm{~V}$ vs $\mathrm{Ag} / \mathrm{AgCl}(3 \mathrm{M})$ was applied to the working electrode, using $0.1 \mathrm{~V}$ intermediate steps.

All measurments have been performed at room temperature $\left(25^{\circ} \mathrm{C}\right)$.

\subsection{Results and discussion}

A typical $\beta$-lactoglobulin adsorption curve measured using SPR is depicted in Figure 7.2 a. Initially, $\beta$-lactoglobulin adsorbs quickly (after 60 seconds of baseline measurement), but after around 3 minutes the surface becomes saturated and the adsorption rate decreases (Figure 7.2 a inset). The maximum adsorbed amount when no potential (NP) or the open circuit potential is applied is comparable with values previously found using ellipsometry $\left(2.7 \mathrm{mg} \cdot \mathrm{m}^{-2}\right.$ ) (Elofsson et al., 1997; Marsh et al., 2002; Rabe et al., 2007). Under a potential of $-0.4 \mathrm{~V}$ proteins adsorb much slower, indicating an increase in adsorption barrier by $6.3 \mathrm{~kJ} \cdot \mathrm{mol}^{-1}$, and the maximum adsorbed amount after $3 \mathrm{~h}$ is reduced to $1.0 \mathrm{mg} \cdot \mathrm{m}^{-2}$ (Figure $7.2 \mathrm{a}$ ).

Activating the potential from open circuit conditions to $-0.4 \mathrm{~V}$ after adsorbing for $1800 \mathrm{~s}$, induces the desorption of some $\beta$-lactoglobulin (Figure $7.2 \mathrm{~d}$ ); the amount of surface bound proteins decreases by up to $15 \%$, which becomes slightly less upon repeated operation (Figure $7.2 \mathrm{e}$ ). The actual amount that can be desorbed depends on the adsorption time. 
a

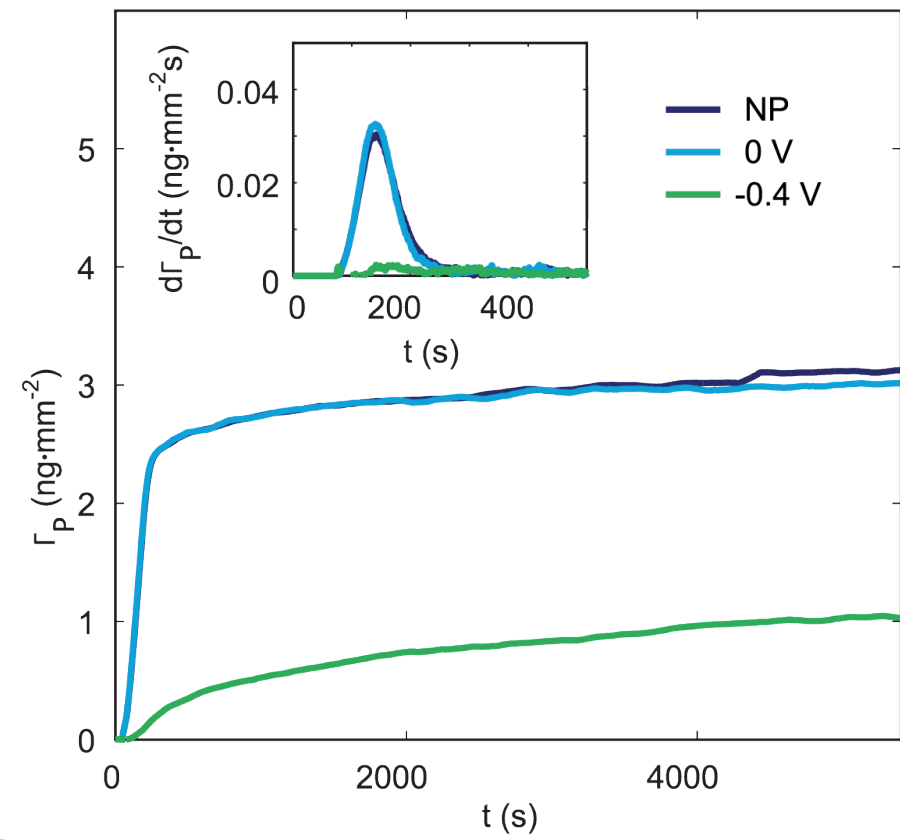

d

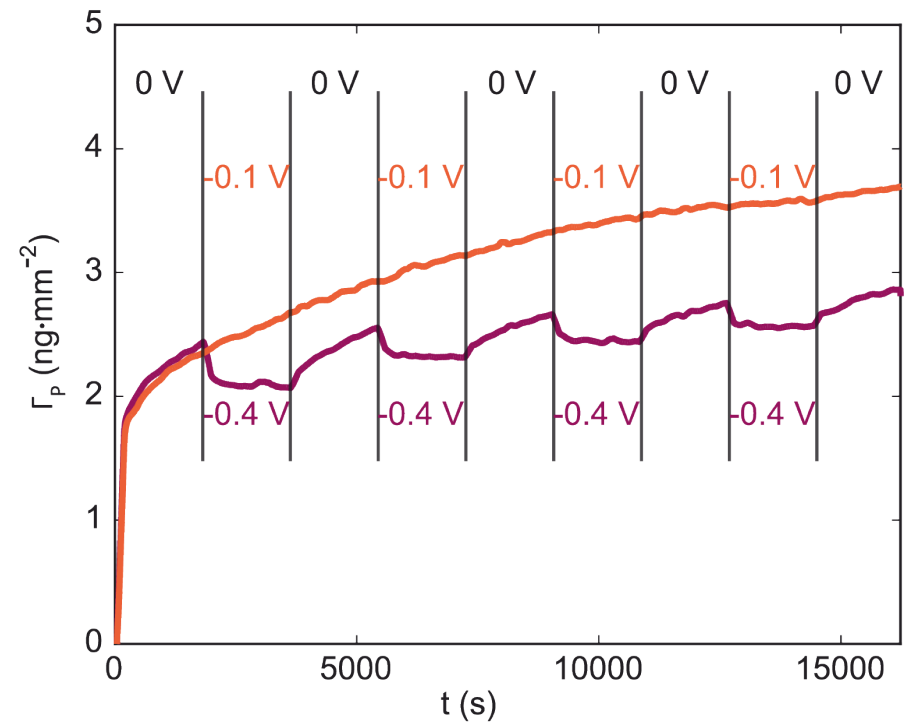

b

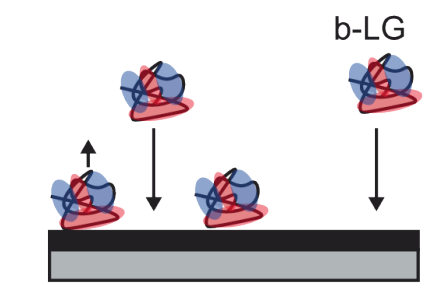

C

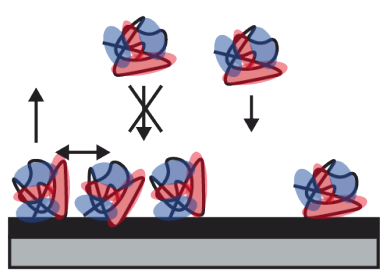

e

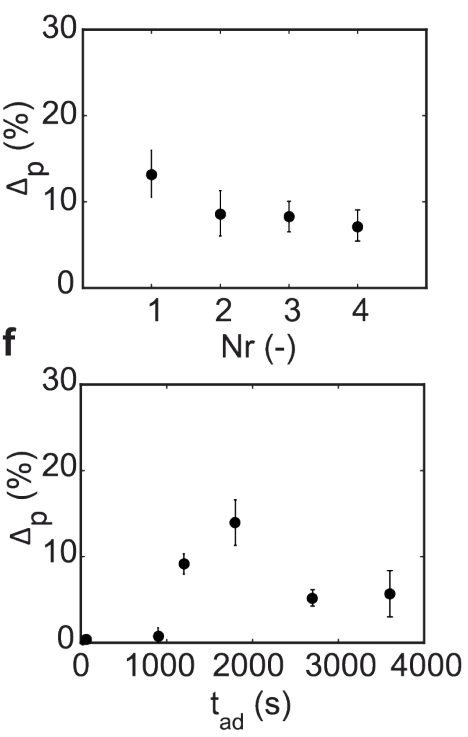

Figure 7.2 Electrochemically modulated protein adsorption and desorption. a Adsorbed amount of protein, and adsorption rate (inset) over time at different applied potentials leading to an extra adsorption barrier of $6.3 \mathrm{~kJ} \cdot \mathrm{mol}^{-1}$ at $-0.4 \mathrm{~V}$ relative to OCP (NP is no potential applied). b Protein adsorption to surface with low coverage. c Protein adsorption to surface with high coverage. d $\beta$-lactoglobulin adsorption at $0 \mathrm{~V}$ and desorption at $-0.1 \mathrm{~V}$ (orange), and $-0.4 \mathrm{~V}$ vs OCP (purple). e Desorption efficiency after repeated adsorption for $1800 \mathrm{~s}$ at $0 \mathrm{~V}$ vs. OCP, and subsequently desorption at $-0.4 \mathrm{~V}$ vs. OCP. $f$ Desorption efficiency of first cycle when $-0.4 \mathrm{~V}$ vs. OCP is applied, depending on different adsorption times.

Three situations can be distinguished (i) at low surface coverage, $\beta$-lactoglobulin adsorbs irreversibly (Figure 7.2 b), (ii) beyond a critical surface coverage the number of contact points per protein decreases due to crowding, which increases the desorption rate (Figure 7.2 c), and (iii) conformational rearrangements of the 
molecules in the adsorption layer result in relaxation and a decreased desorption rate constant (Rabe et al., 2007), as also illustrated in Figure 7.2 f. Which role the various forces play is explained next.

The repulsive forces between the gold surface and silicon probe, measured with AFM, increase upon applying a more negative potential (Figure 7.3 a). If we switch the potential from $0 \mathrm{~V}$ to $-0.4 \mathrm{~V}$, repulsion increases from $0.2 \mathrm{mN} \cdot \mathrm{m}^{-1}$ to $9 \mathrm{mN} \cdot \mathrm{m}^{-1}$ at $3 \mathrm{~nm}$ from the surface, and the distance over which the repulsive forces decay to practically zero (cutoff value: $0.01 \mathrm{mN} \cdot \mathrm{m}^{-1}$ ) increases from around $5 \mathrm{~nm}$ to $26 \mathrm{~nm}$.

a

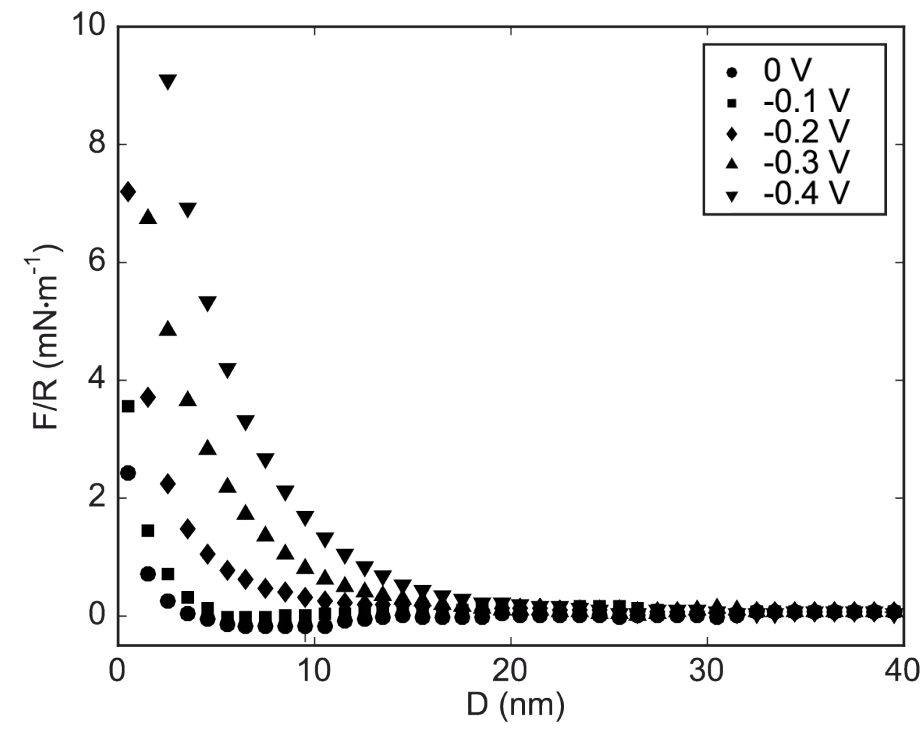

b

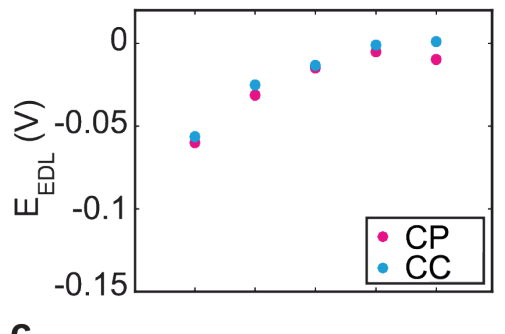

C

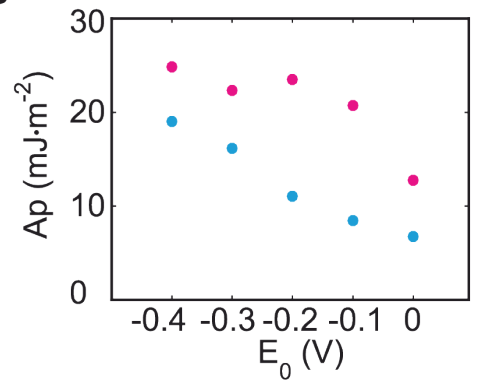

Figure 7.3 Surface interactions between gold electrode and silicon probe. a Measured energy-distance curves between truncated silicon probe and gold electrode biased at potentials ranging from 0 to $-0.4 \mathrm{~V}$ vs OCP. b Double layer potential EEDL, and $\mathbf{c}$ Hydration repulsion amplitude $A_{p}$ obtained after fitting interaction energy curves (Figure S7.4) either using constant potential (CP, blue) or constant charge (CC, red) boundary conditions.

Fitting the energy distance curves with DLVO theory allows distinction between electrostatic and van der Waals contributions, but also hydration interactions should be considered. The change in electrostatic potential $\psi$ in the diffuse double layer over distance $z$ follows from the nonlinear Poisson-Boltzmann equation (Equation 7.2).

$$
\frac{d^{2} \psi}{d z^{2}}=\kappa^{2} \frac{k T}{e} \sinh \left(\frac{e}{k T} \psi\right)
$$


Here $\kappa^{-1}$ is the Debye screening length, $e$ the elementary charge, $\varepsilon$ the relative dielectric constant (78.54), $\varepsilon_{0}$ the permittivity of vacuum and $k$ the Boltzmann constant. The excess of ions near the electrode (Figure 7.4 b) compensating the surface charge, lead to repulsion when a second negatively charged object approaches (Bard and Faulkner, 1944). This repulsion was calculated using Equation 7.3 and 7.4 (results shown in Figure $7.4 \mathrm{c}$ and $\mathbf{d}$, are for constant potential and constant charge boundary conditions, respectively).

$$
\begin{gathered}
\Pi_{E l}(D)=2 n_{0} k T\left[\cosh \left(\frac{e \psi}{k T}\right)-1\right]-\frac{\epsilon \epsilon_{0}}{2}\left(\frac{d \psi}{d z}\right)^{2} \\
\Phi_{E l}=\Pi_{E l}(D) \pi R_{p}+2 \pi \int_{R}^{\infty} \Pi_{E l}\left(D+\frac{r_{p}-R_{p}}{\tan \alpha}\right) d r
\end{gathered}
$$

Equation 7.3 gives the pressure in the gap (disjoining pressure $\Pi_{E I}$ ) between two flat surfaces, with $n_{0}$ the number of ions in the bulk $\left(\mathrm{m}^{-3}\right)$, and $D$ the distance between the two plates. For calculation of the electrostatic interaction energy $\left(\Phi_{E I}\right)$ between the adsorbing surface and the probe (Equation 7.4), the shape of the probe has been taken into account: $R_{p}$ and $r_{p}$ are radii at different locations on the cone as indicated in Figure $7.1 \mathrm{c}$, and $\alpha$ is the cone angel. Since the silicon probe has an oxidized surface layer, we used $-0.06 \mathrm{~V}$ for the surface potential of silica (Ebeling et al., 2011) and used the surface potential of the gold electrode as fit parameter. At constant potential boundary conditions, a maximum can be observed for the electrostatic interaction, leading to attractive forces at distances below $8 \mathrm{~nm}$ (Figure 7.4 c), this is due to charge reversal on the boundary (Figure S7.2 and S7.3) (Hillier et al., 1996).

The gold-water-silica system has a rather high Hamaker constant $\left(5.7 \cdot 10^{-20} \mathrm{~J}\right.$ (Barten et al., 2003a)), which leads to strong attraction at distances shorter than 10-15 nm (see Figure 7.4 e, calculated using Equation 7.5 and 7.6 (Ebeling et al., 2011; Israelachvili, 2011)).

$$
\Pi_{V d W}(D)=-\frac{A_{H}}{6 \pi D^{3}}
$$




$$
\Phi_{V d W}=\Pi_{V d W}(D) \pi R_{p}+2 \pi \int_{R}^{\infty} \Pi_{V d W}\left(D+\frac{r_{p}-R_{p}}{\tan \alpha}\right) d
$$

This attraction is, however, rarely detected in energy-distance curves as measured here (Figure 7.3 a) (Giesbers et al., 2002), which can be related to strong hydration forces acting at close proximity to the surface. Thus the contribution of hydration repulsion was calculated using Equation 7.7 (Kilpatrick et al., 2013) and 7.8, and results for different hydration repulsion amplitudes $A_{p}$ at a decay length of $1.29 \mathrm{~nm}$ are given in Figure $7.4 \mathrm{f}$.

$$
\begin{gathered}
\Pi_{H y}(D)=\frac{A_{p}}{D} e^{-\frac{D}{\lambda}} \\
\Phi_{H y}=\Pi_{H y}(D) \pi R_{p}+2 \pi \int_{R}^{\infty} \Pi_{H y}\left(D+\frac{r_{p}-R_{p}}{\tan \alpha}\right) d r
\end{gathered}
$$

To fit the energy distance curves in Figure $7.3 \mathrm{a}$, the different interaction contributions were added up. For a good fit we had to shift the data points by $5 \mathrm{~nm}$ to the right (Figure S7.4). A physical justification for this is the surface roughness of both the polished polycrystalline gold electrode and the blunted AFM probe, affecting the precise onset of the interactions (Barten et al., 2003a). In addition, factors such as the angle of the flattened cone surface with respect to the gold substrate and the exact value of its radius add to the fitting uncertainties. The double layer potential derived from the measured force curves (Figure 7.3 b) is more negative with increasing negative applied potential (leading to more electrostatic repulsion). When applying $-0.4 \mathrm{~V}$ vs OCP a double layer potential of $-0.06 \mathrm{~V}$ was achieved being a bit larger as compared to what was determined by Barten et al. (2003a) using a colloidal silica probe in a $1 \mathrm{mM} \mathrm{KNO}_{3}$ electrolyte at $\mathrm{pH} 4.7$ when applying $-0.3 \mathrm{~V}$ vs $\mathrm{Ag} / \mathrm{AgCl}$. Furthermore, the hydration repulsion amplitude increases (Figure $7.3 \mathrm{c}$ ) indicating stronger hydration repulsion at more negative potentials. 


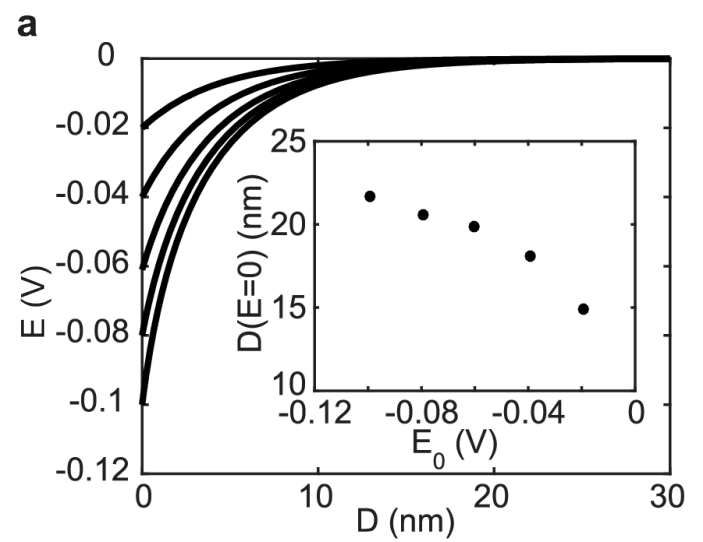

C

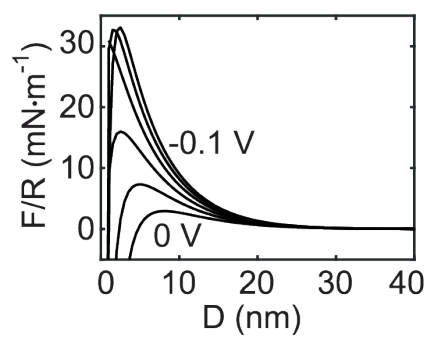

d

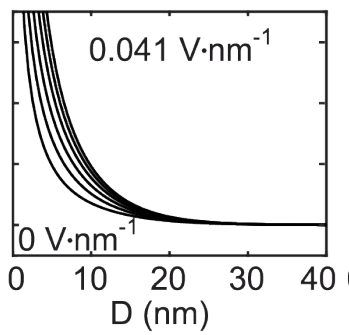

b

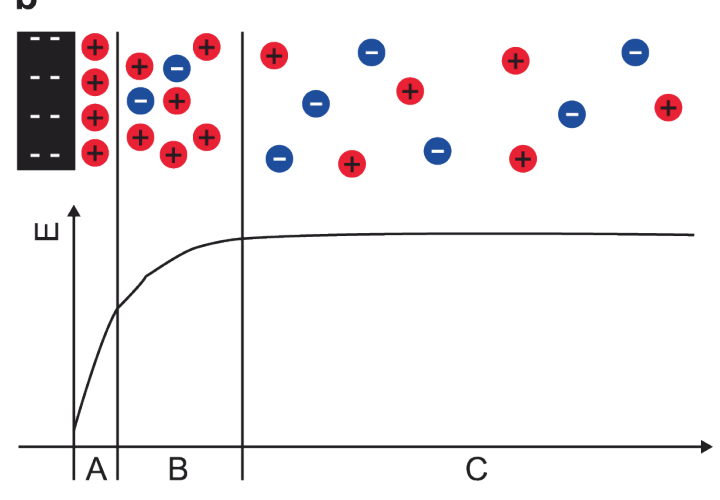

e

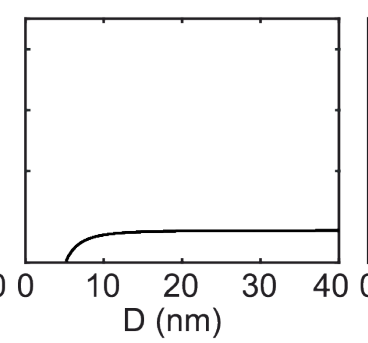

$\mathbf{f}$

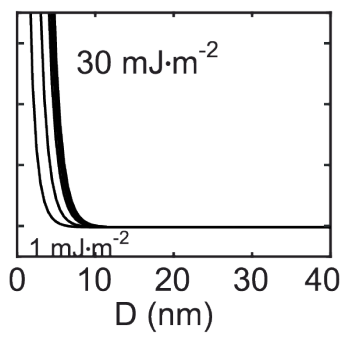

Figure 7.4 a Double layer potential $(E)$ profile from the electrode interface ( $E_{0}$ between -0.1 and $-0.02 \mathrm{~V}$ ) to the electrolyte solution. Inset: Distance at which the potential drops to $0 \mathrm{~V}$ (cutoff: $-5 \cdot 10^{-4} \mathrm{~V}$ ). b Schematic of ion distribution at electrode interface within the electric double layer and potential profile at interface. c Electrostatic interaction energy determined for constant potential boundary conditions, gold electrode was held at $0,-0.2,-0.4,-0.6,-0.8$ or $-1.0 \mathrm{~V}$ and silica probe set at $-0.06 \mathrm{~V}$. d Constant charge boundary conditions were determined using Graham's equation for values between 0 and $0.041 \mathrm{~V} \cdot \mathrm{nm}^{-1}$ for the gold electrode, and $-0.0173 \mathrm{~V} \cdot \mathrm{nm}^{-1}$ for the silica surface. $\mathbf{e}$ Van der Waals attraction for a Hamaker constant of $5.7 \cdot 10^{-20} \mathrm{~J}$, and $\mathbf{f}$ hydration repulsion for hydration repulsion amplitude between 1 and $30 \mathrm{~mJ} \cdot \mathrm{m}^{-2}$ with decay length of $1.29 \mathrm{~nm}$.

The hydration repulsion effects relate to a change in surface wetting, which is also determined experimentally at meso scale through contact angle measurements (Figure 7.5 a, b and S7.5). The advancing contact angle decreased from 80 to $72^{\circ}$ when shifting from 0 to $-0.5 \mathrm{~V}$. This implies that the interaction between water and surface becomes stronger, leading to increasing work of water adhesion ( $W$, Equation 7.9), and a more negative free energy of wetting per mole of surface sites $\left(\Delta G_{E}\right.$, Equation 7.10) (Vogler, 2012),

$$
\begin{gathered}
W=\gamma_{l v}\left(1+\cos \theta_{a}\right) \\
\Delta G_{E}=\left(\frac{R T}{3}\right) \ln \left[\frac{\left(1-\cos \theta_{a}\right)^{2}\left(2+\cos \theta_{a}\right)}{4}\right]
\end{gathered}
$$

where $\gamma_{V}$ is the surface tension of water and $\theta_{a}$ the advancing contact angle (Figure 7.5 b). Usually, $63^{\circ}$ is assumed as threshold advancing contact angle between 
hydrophilic and hydrophobic, since then the water orientation correlates with the $70: 30$ hydrogen-bonding-to-dispersion-force contributions for self-association of water (Vogler, 2012). For a gold substrate, the majority of water molecules lays flat or perpendicular on the surface contributing with at least one $\mathrm{H}$-atom to the hydrogen bond network. Thus can be concluded that a gold surface has more $\mathrm{H}$-donors than $\mathrm{H}$-acceptors and with an increasing negative potential this ratio is further distorted leading to a smaller contact angle and more hydration repulsion (Besseling, 1997; Velasco-Velez et al., 2014).

a

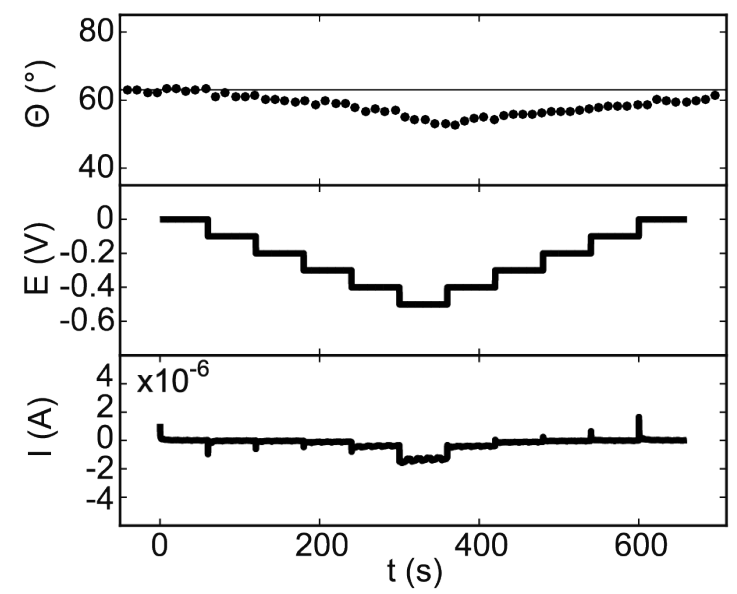

b

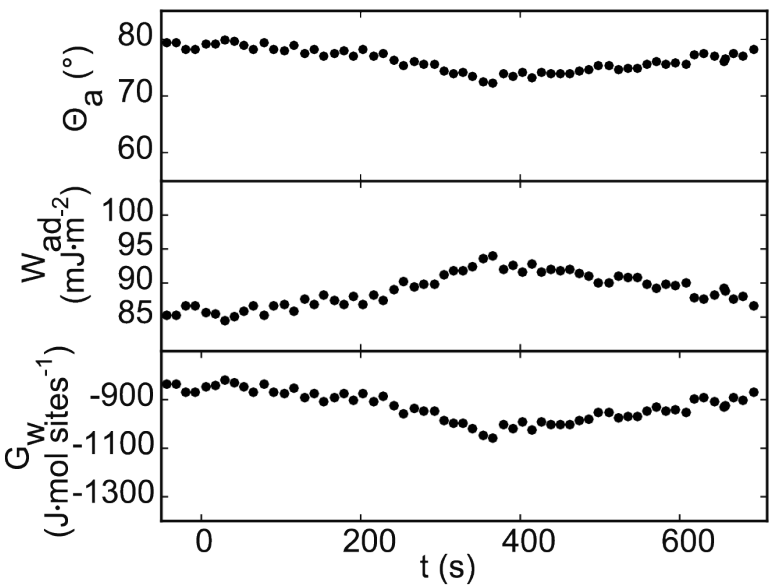

Figure 7.5 Dynamic contact angle of water at the gold electrode as a function of applied potential. a Average dynamic contact angle (top) depending on applied potential (middle) and current response (bottom). b Advancing contact angle (top) and resulting work of water adhesion (middle) and free energy of wetting a mole of surface sites (bottom).

All these findings together indicate that overlapping electric double layers and the arrangement of water molecules near the surface both contribute to repulsive forces at the interface. Furthermore, we conclude that both force contributions can be influenced by an externally applied potential increasing the repulsive forces at increasingly negative applied potential. This in turn influences the adsorption free energy, and thus not only decreases protein adsorption but also induces protein desorption especially when close to critical surface coverage. This effectively prevents stronger adsorption at high surface coverage.

The latter, as reported and analyzed for the first time in this manuscript, and it is essential when considering regeneration of electrode surfaces for adsorptive separation processes or electrochemical sensor interfaces. The findings indicate that in order to induce protein desorption by applying by an electric potential either an 
increase in the double layer potential can be used or an increase in hydration repulsion. Both can be influenced by an increase in the applied potential but also by choosing electrode materials with a low impedance, to increase the potential drop in the electrolyte (double layer potential). Furthermore, electrode materials that show good electrowetting properties should be favored.

\subsection{Conclusion}

We present a unique combination of techniques to study the influence of the surface potential of a solid substrate on protein adsorption as well as desorption. Upon changing the surface potential with a negative electric bias of $-0.4 \mathrm{~V}$ with respect to OCP the adsorption barrier increased by $6.3 \mathrm{~kJ} \cdot \mathrm{mol}^{-1}$ due to an increase in electrostatic and hydration repulsion leading to reduced and slower protein adsorption. Furthermore, the increase in the double layer potential, and work of water adhesion also triggered protein desorption. These are crucial findings that help to elucidate the complex mechanisms behind protein ad- and desorption, and how both can be modulated by the surface potential, and thus applied in the design of innovative biosensors as well as protein separation technologies that can be loaded and regenerated by the 'switch of a button'. 


\subsection{Supporting information}
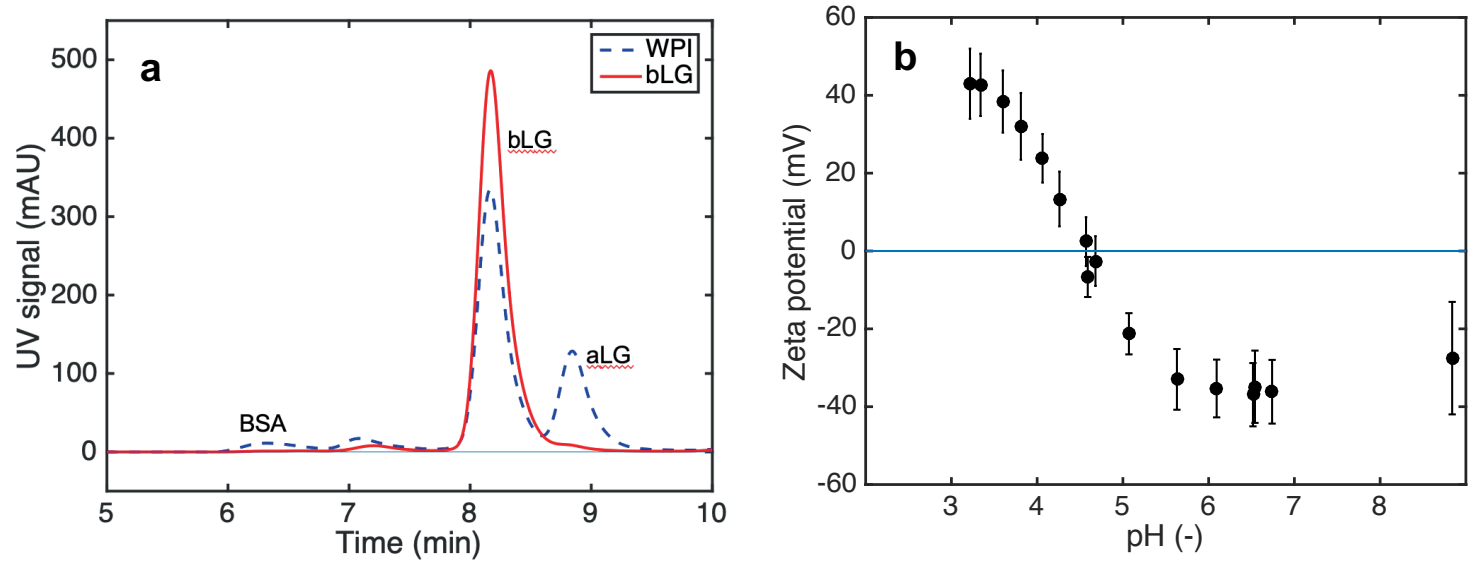

Figure S7.1. a HPLC retention peaks of the original whey protein isolate, and the purified $\beta$-lactoglobulin. $\mathbf{b}$ Zeta potential of $\beta$-lactoglobulin as function of $\mathrm{pH}$.
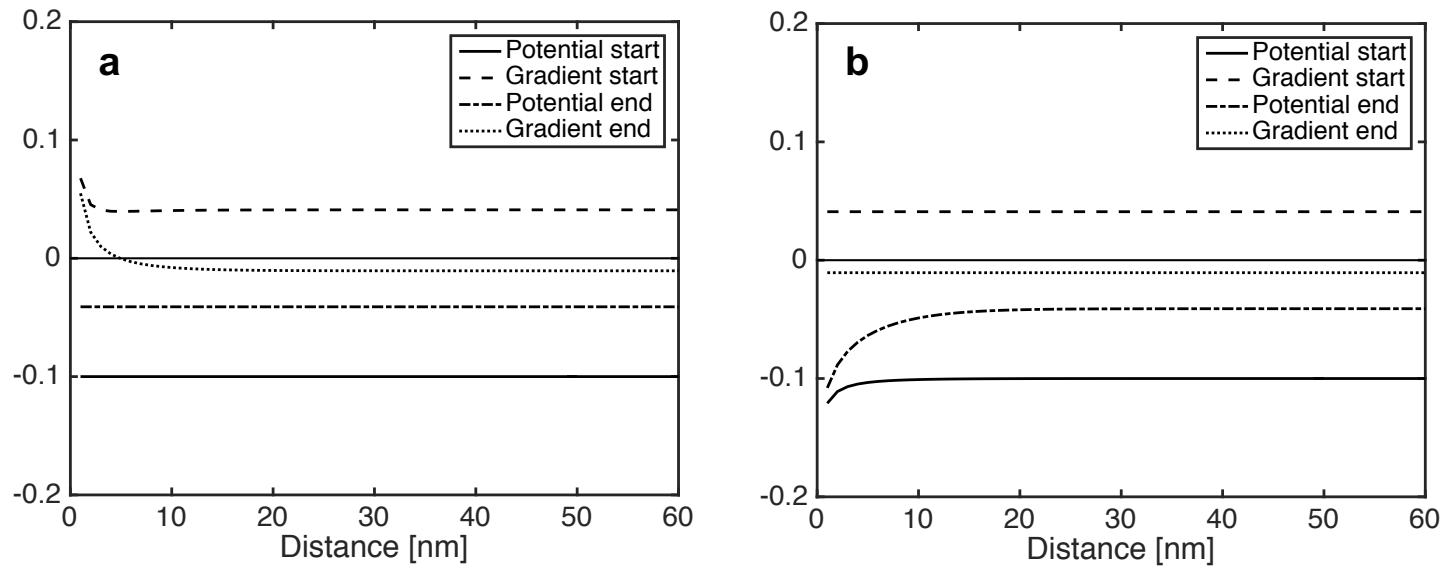

Figure S7.2. Boundary conditions for different distances between the surfaces. a Constant potential boundary conditions. $\mathbf{b}$ Constant charge boundary conditions. Solid line at $\mathrm{y}=0$ serves to guide the eye. Potential start indicates the potential on the left surface where as potential end indicates the potential on the right surface. The same notation counts for the gradient. 

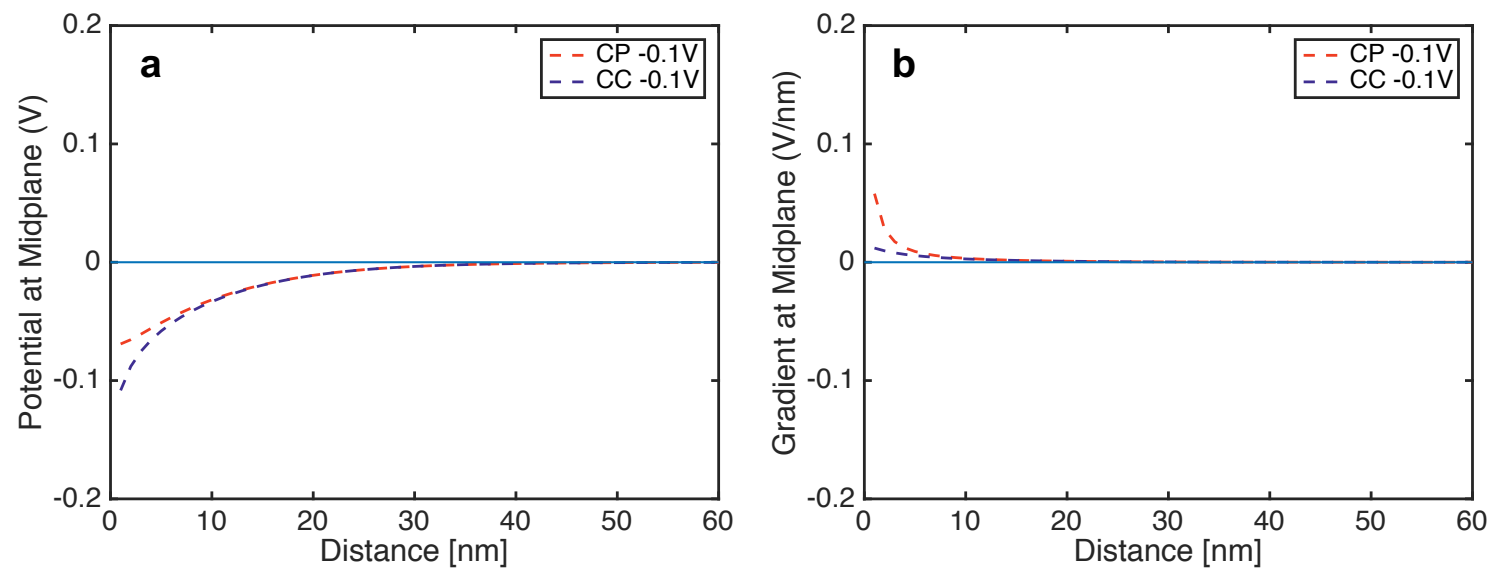

Figure S7.3. a Potential and $\mathbf{b}$ gradient at mid plane as a function of the distance between the surfaces at constant potential and constant charge boundary conditions.

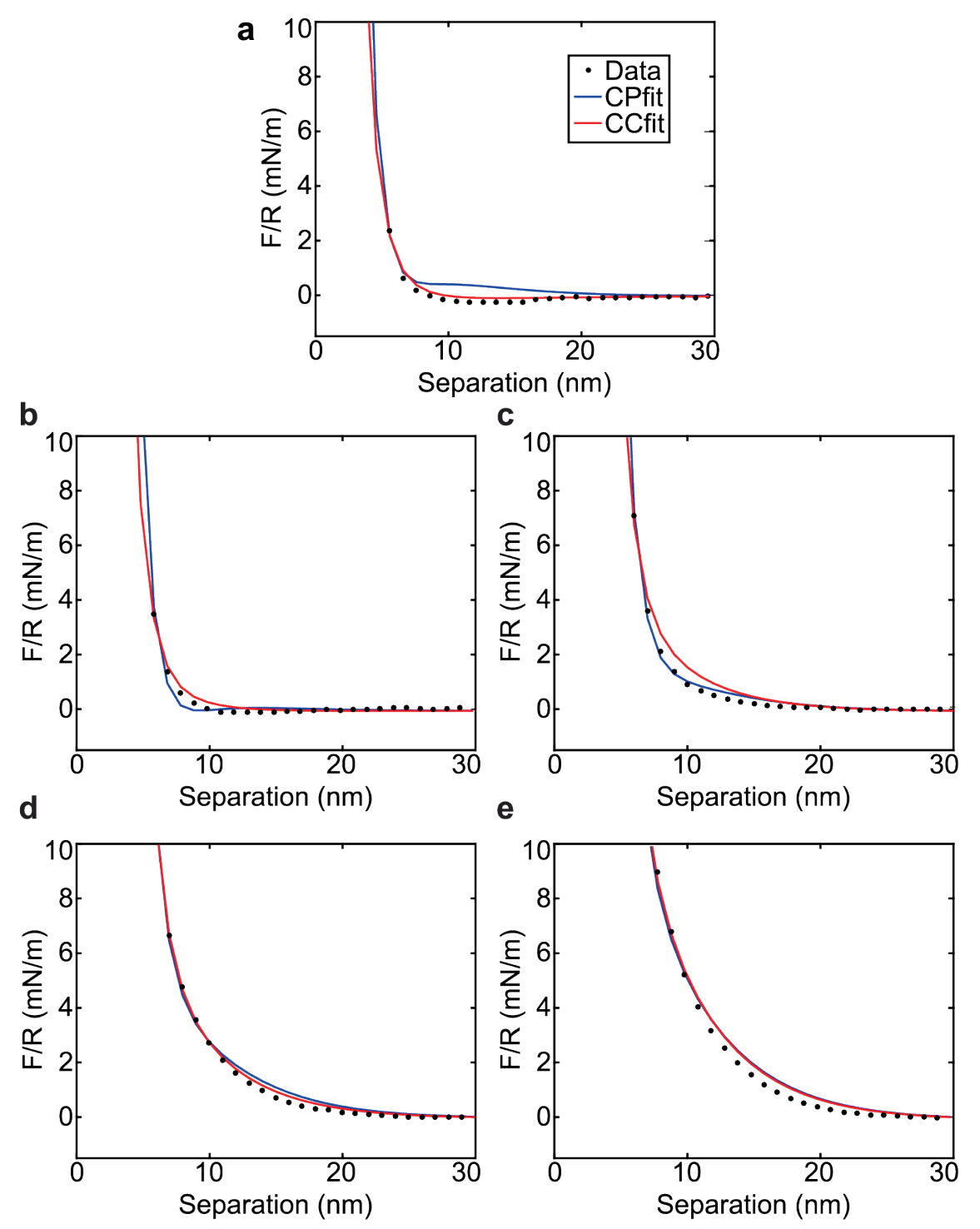

Figure S7.4. Constant charge and constant potential fits to AFM force curves a-e $0,-0.1,-0.2,-0.3$, $-0.4 \mathrm{~V}$ vs. OCP, respectively. 


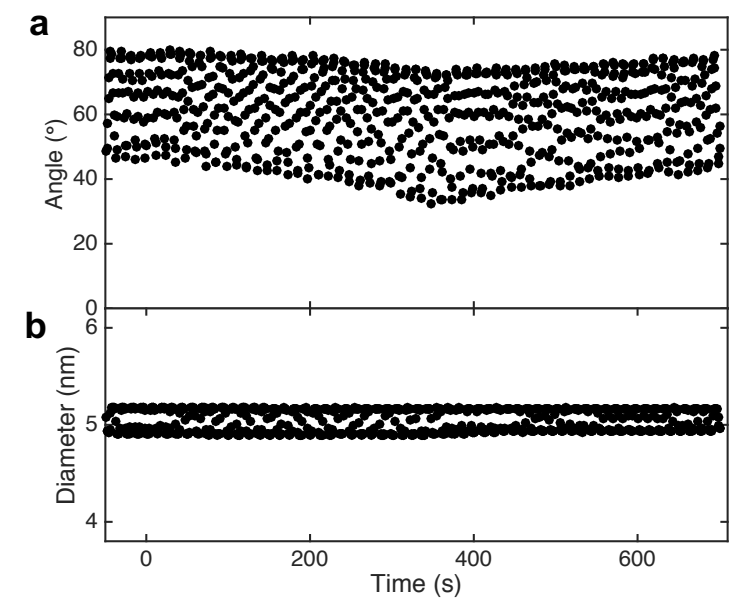

Figure S7.5. a Dynamic contact angle of water on gold electrode obtained while changing the applied potential as can be seen in the manuscript in Figure $\mathbf{7 . 5}$ and $\mathbf{b}$ corresponding drop diameters. 



\section{CHAPTER 8}

\section{SIMULTANEOUS SILICON OXIDE GROWTH}

\section{AND ELECTROPHORETIC DEPOSITION OF GRAPHENE OXIDE}

During electrophoretic deposition of graphene oxide (GO) sheets on silicon substrates, not only deposition but also simultaneous anodic oxidation of the silicon substrate takes place, leading to a three-layered material. SEM images reveal the presence of GO sheets on the silicon substrate, and this is also confirmed by XPS, albeit that the carbon portion increases with increasing emission angle, hinting at a thin carbon layer. With increasing applied potential and increasing conductivity of the GO solution the carbon signal decreases, while the overall thickness of the added layer formed on top of the silicon substrate increases. Through XPS spectra in which the Si2p peaks shifted under those conditions to $103-104 \mathrm{eV}$ we were able to conclude that significant amounts of oxygen are present, indicative of formation of an oxide layer. This leads us to conclude that GO can be deposited using electrophoretic deposition, but that at the same time silicon is oxidized, which may overshadow effects previously assigned to GO deposition.

This chapter has been published as:

P. A. Fritz, S. C. Lange, M. Giesbers, H. Zuilhof, R. M. Boom, and K. Schroën. Simultaneous silicon oxide growth and electrophoretic deposition of graphene oxide. Langmuir 35, 3717-3723 (2019). 


\subsection{Introduction}

Transparent electrodes are used in many electronic devices such as touch screens (Wang et al., 2012), thin-film photovoltaics (Yin et al., 2014), and transistors (Huang et al., 2012). Reduced graphene oxide (GO) thin films are promising cheaper alternatives (Kumar et al., 2015; Wöbkenberg et al., 2011) to the current standard indium-doped tin oxide (ITO). Graphene oxide is a 2D material synthesized by chemical exfoliation of graphite, (Dreyer et al., 2010) that in its original state shows low conductivity, but when reduced by either thermal, (Yang et al., 2012) chemical (Park et al., 2011) or electrochemical methods, (Shao et al., 2010; Yu et al., 2013; Zhang et al., 2012) the conductivity can become as high as $100 \mathrm{~S}^{\circ} \mathrm{cm}^{-1}$ (Gao, 2015). Further, their thermal stability, flexibility, and light transmittance of $80-85 \%$ at a wavelength of $550 \mathrm{~nm}$ make reduced GO thin films interesting candidates for flexible transparent electrodes (Gao et al., 2013; Karthick et al., 2013; Pham et al., 2010; Yin et al., 2010).

Many techniques to produce GO thin films have been proposed, amongst others spray-coating, (Chan Lee et al., 2015; Pham et al., 2010) spin-coating, (Becerril et al., 2008; Watcharotone et al., 2007) and electrophoretic deposition (An et al., 2010; Chavez-Valdez et al., 2013; Ishikawa et al., 2012; Liang et al., 2013). When applying the latter technique, GO sheets migrate along an electric field towards the anode due to the negatively charged oxygen groups (typically carboxyl and hydroxyl groups) present on the lateral plane and edges of the GO sheets. Once they reach the surface of the anode, the charged sheets deposit and form a new layer on the electrode, and further reduction can take place (Chavez-Valdez et al., 2013). This technique can be applied to many conductive substrates such as copper, nickel, aluminum and stainless steel, and is not limited to planar shapes (Chavez-Valdez et al., 2013; Hasan et al., 2010; Lake et al., 2012; Park and Park, 2014; Vlachova et al., 2015). A significant advantage of electrophoretic deposition is thus that the GO is directly reduced during the deposition process (An et al., 2010).

Apart from the metallic substrates mentioned above, also silicon has been used as substrate for electrophoretic deposition of GO, (An et al., 2010; Ishikawa et al., 2012; Liang et al., 2013) with e.g. the aim to reduce wear and tear in micro- and nanoelectromechanical systems (MEMS/NEMS). This use of GO has led to an order of magnitude reduction in both friction and wear volume, as compared to bare silicon, 
making GO a promising solid lubricant. For this purpose, layer thicknesses of up to $402 \mathrm{~nm}$ have been reported, (Liang et al., 2013) based primarily on ellipsometry and cross-sectional SEM images. In these estimates, the deposited GO layer has, however, to the best of our knowledge, never been distinguished from any anodically oxidized silicon layer underneath. To properly evaluate and understand the performance of the layer, a compositional analysis is, of course, required.

The anodic oxidation of silicon at the interface of an electrolyte has been described with the following reactions (Zhang, 2001).

$$
\begin{aligned}
& \mathrm{Si}+\mathrm{H}_{2} \mathrm{O}+h \rightarrow \mathrm{SiOH}+\mathrm{H}^{+} \\
& 2 \mathrm{SiOH} \rightarrow \mathrm{Si}_{2} \mathrm{O}+\mathrm{H}_{2} \mathrm{O} \\
& 2 \mathrm{Si}_{2} \mathrm{O} \rightarrow \mathrm{SiO}_{2}+3 \mathrm{Si}
\end{aligned}
$$

The majority of the oxygen atoms in an anodically grown oxide layer originate from water that is present at or is generated by redox reactions of salt or solvent molecules at the electrode interface. Water only enters the most outer layer, where proton loss occurs, which leads to hydroxyl or oxide ions traveling further into the silicon structure along the electric field (Zhang, 2001). In this paper, we describe the influence of several electrophoretic deposition parameters on the deposition of GO and growth of anodic silicon oxide, using SEM, XPS, ellipsometry, and color analysis.

\subsection{Materials and methods}

\subsubsection{Electrophoretic deposition of graphene oxide}

Prior to the coating process, the GO solution (Sigma-Aldrich, $4 \mathrm{mg} \cdot \mathrm{mL}^{-1}$ ) with a conductivity of $7 \mu \mathrm{S} \cdot \mathrm{cm}^{-1}$ was sonicated and depleted of oxygen using a $\mathrm{N}_{2}$ purge for $30 \mathrm{~min}$. The silicon substrates $(1 \times 4 \mathrm{~cm})$ were cleaned with piranha acid (3:1) for $15 \mathrm{~min}$. Later, two silicon substrates were immersed into the deposition cell at a distance of $10 \mathrm{~mm}$, and a constant potential of $5,20,30$ or $60 \mathrm{~V}$ was applied for $1 \mathrm{~h}$ (Power supply EPS 1001, Amersham Pharmacia Biotech, USA). To increase the conductivity of the GO solution, 10 or $1000 \mathrm{mM}$ sodium sulfate was added to reach conductivities of 16 and $485 \mu \mathrm{S} \cdot \mathrm{cm}^{-1}$, respectively. Next to $1 \mathrm{~h}$, silicon substrates were also coated for 0.5 and $2 \mathrm{~h}$ at $20 \mathrm{~V}$. As blanks, a silicon substrate was immersed in the 
GO solution for $1 \mathrm{~h}$ without applying a potential, and another silicon substrate was exposed to a sodium sulfate solution with a conductivity of $7 \mu \mathrm{S} \cdot \mathrm{cm}^{-1}$ but without any GO present, while applying a potential of $20 \mathrm{~V}$ for $1 \mathrm{~h}$.

Since GO solutions can vary in sheet size, oxidation degree and conductivity, as comparison, another $\mathrm{GO}$ solution $(\mathrm{GO}(2))$ was synthesized from graphite powder (Sigma-Aldrich, $<20 \mu \mathrm{m}$ ) by a modified Hummers method (Wang et al., 2017). The resulting GO solution ( $1 \mathrm{mg} \cdot \mathrm{mL}^{-1}$ ) with a conductivity of $14 \mu \mathrm{S} \cdot \mathrm{cm}^{-1}$ had a zeta potential of $-32.5 \mathrm{mV}$ (Zetasizer Nano-ZS, Malvern instruments, UK) and the d-spacing of the freeze-dried sample (FreeZone 2.5 Plus, Labconco, USA) was measured to be $8.62 \AA$ using X-ray diffraction spectroscopy (D8 Advanced, Bruker, USA). Silicon substrates were coated as described above at $30 \mathrm{~V}$ for $1 \mathrm{~h}$. All samples were produced in triplicate.

\subsubsection{Spin coating of $\mathrm{Si}$ substrates}

$\mathrm{GO}(2)$ solution was sonicated for $30 \mathrm{~min}$ and the silicon substrate $(1 \times 4 \mathrm{~cm})$ was cleaned with piranha acid (3:1) for $15 \mathrm{~min}$ and etched with $3.5 \% \mathrm{HF}$ for $4 \mathrm{~min}$. Subsequently $750 \mu \mathrm{L}$ GO solution was put onto the substrate for $1 \mathrm{~min}$ and spun at $600 \mathrm{rpm}$ for $2 \mathrm{~min}$ and $2000 \mathrm{rpm}$ for $1 \mathrm{~min}$. As comparison the same procedure was conducted without HF etching. All samples were produced in triplicate.

\subsubsection{Preparation of reference silicon substrates}

A silicon substrate $(1 \times 4 \mathrm{~cm})$ was first cleaned for $15 \mathrm{~min}$ in piranha acid and subsequently etched with $3.5 \% \mathrm{HF}$ for $4 \mathrm{~min}$ to remove oxygen. Another silicon wafer was heated to $1000^{\circ} \mathrm{C}$ for $1 \mathrm{~h}$ in a lab furnace (Thermo Fischer Scientific), to achieve a thermally grown oxide layer at the outside of the substrate. All samples were produced in triplicate.

\subsubsection{Surface characterization.}

The overall thickness of anodic silicon oxide and GO was characterized at three different positions on each sample (triplicate) using static angle ellipsometry at a wavelength of $632.8 \mathrm{~nm}$ and an incidence angle of $70^{\circ}$ (SE400, Sentech, Germany). Both the overall layer thickness and the refractive index were used as fitting parameters and average values with according standard deviations were calculated. 
Static $\left(80^{\circ}\right)$ and angle-resolved $\left(0-80^{\circ}\right.$ between sample and detector) X-ray photoelectron spectra were measured (XPS, JPS-900 JEOL, Japan) to evaluate the chemical composition of each surface. The element concentrations on the surfaces of the individual sample sets were averaged and the according standard deviations were calculated. Scanning electron microscopy (SEM) images of the top surface $(2,000$ and $10,000 \mathrm{x})$ and the cross section $(350,000 \mathrm{x})$ were taken using $2 \mathrm{kV}$ acceleration voltage (SEM, Magellan 400, FEI, USA). AFM surface topography images were acquired by an Asylum Research MFP-3D SA AFM (Oxford Instruments, United Kingdom). A sharp knife was used to scratch the surfaces. The scratched surfaces were rinsed with water.

\subsection{Results and discussion}

During electrophoretic deposition, GO sheets migrated towards the positively charged electrode (anode) and deposited on the silicon substrate. After the coating process, the resulting modified substrates had different colors depending on the process potential, solution conductivity and coating time used, varying between brownish, grey and blue (Figure 8.1, inserts).

In literature, these colors were directly related to the GO thickness, and were implied to correlate with a deposition of hundreds of nanometers of GO (Liang et al., 2013). The SEM images in Figure $\mathbf{8 . 1}$ and S8.1, however, reveal sharp contours of individual GO sheets and the visibility of the underlying substrate is first decreasing while going from 5 to $20 \mathrm{~V}$ and then increasing with further increasing coating potential (Figure 8.1 a-e; see also Figure $\mathbf{S 8 . 1}$ a and $\mathbf{b}$ in the Supporting Information), conductivity of the GO solution (Figure $8.1 \mathrm{f}$ and $\mathrm{g}$; see also Figure S8.1 $\mathrm{c}$ and $\mathrm{d}$ ) and coating time (Figure $\mathbf{8 . 1} \mathbf{h}$ and $\mathbf{i}$; see also Figure $\mathbf{S 8 . 1} \mathrm{e}$ and $\mathbf{f}$ ). For example, on the sample coated at $60 \mathrm{~V}$ only a few individual sheets can be found (Figure S8.1 b), thus the blue color of the surface is most likely not caused by the deposited GO, but expected to be related to an anodically grown silicon oxide layer (Henrie et al., 2004).

The presence of silicon oxide was confirmed using XPS analysis. In all samples a silicon, oxygen and carbon peak can be detected (Figure 8.2 a and S8.2). While native silicon has a Si2p peak at $99 \mathrm{eV}$ (Figure $\mathbf{8 . 3} \mathrm{d}$ and $\mathbf{e}$, bottom), the silicon peaks of the GO-coated silicon samples are between 103 and $104 \mathrm{eV}$ (Figure $8.2 \mathbf{f}$ and Figure S8.5). This is comparable to the Si2p peak position of a thermally oxidized 
silicon substrate (Figure $\mathbf{8 . 3} \mathrm{d}$ and $\mathrm{e}$, top) and of a sample exposed to a sodium sulfate solution for $1 \mathrm{~h}$ while applying $20 \mathrm{~V}$ (Figure S8.9). As a reference, $\mathrm{GO}$ sheets can also be spin coated onto an etched silicon substrate, but in this case the Si2p position stays at $99 \mathrm{eV}$, because no silicon oxidation occurs during the process (Figure S8.8).
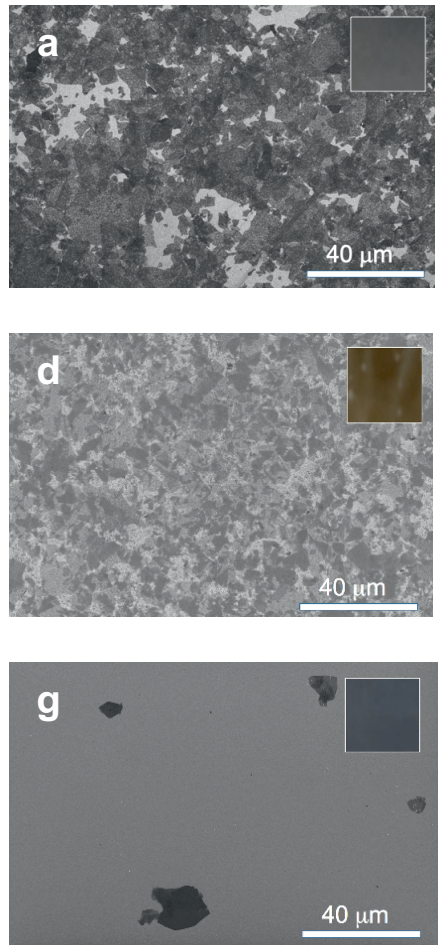
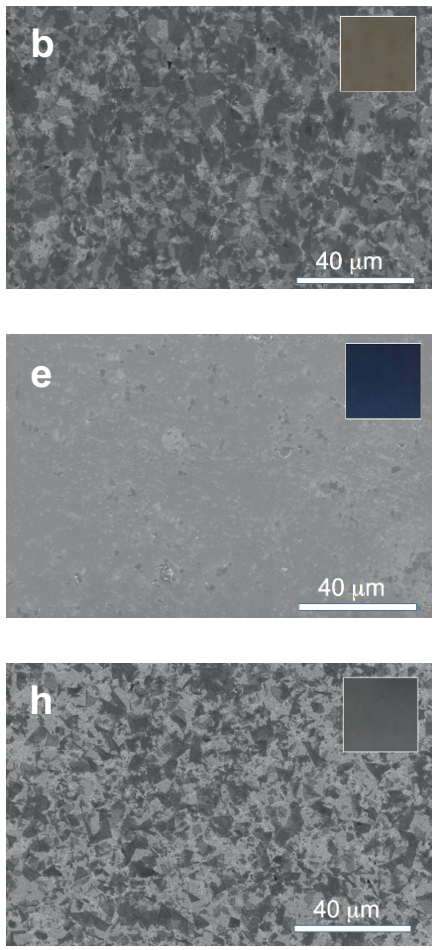
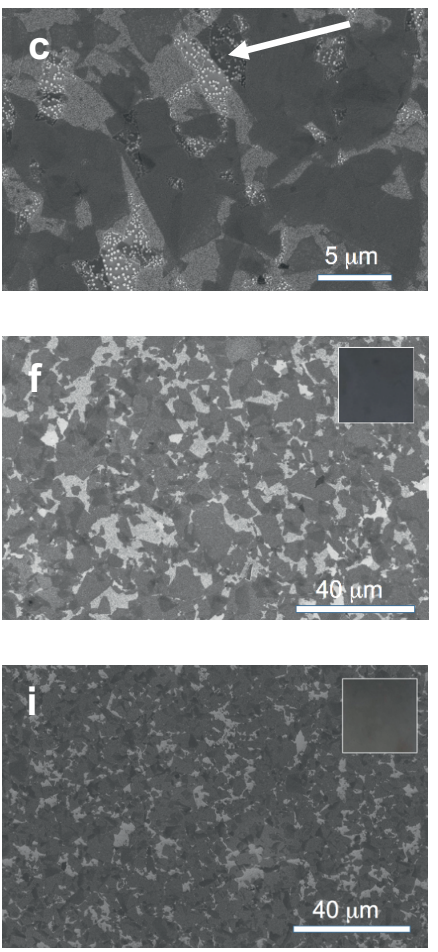

Figure 8.1. SEM images of samples coated for $60 \mathrm{~min}$ at $5(\mathbf{a}), 20(\mathbf{b}, \mathbf{c}), 30$ (d) and $60 \mathrm{~V}(\mathbf{e})$ with a solution conductivity of $7 \mu \mathrm{S}^{\cdot} \mathrm{cm}^{-1} ; 60 \mathrm{~min}$ at $20 \mathrm{~V}$ with a solution conductivity of 16 (f) and $485 \mu \mathrm{S}^{\cdot} \mathrm{cm}^{-1}$ (g); and $7 \mu \mathrm{S}^{\cdot} \mathrm{cm}^{-1}$ for 30 (h) and 120 (i) min (Magnification: $2000 \mathrm{x}$, except b, which is 10,000 x). Arrow in c indicates $\mathrm{MnO}_{2}$ crystals.

The high O/C ratio of 1.6 in the XPS wide scan of the sample coated at $20 \mathrm{~V}$ for $1 \mathrm{~h}$ (Figure $\mathbf{8 . 2}$ a) reveals the presence of more oxygen-containing species on the surface next to GO that has an $\mathrm{O} / \mathrm{C}$ ratio of 0.4 (Figure $8.3 \mathrm{a}$ ). While the $\mathrm{O} 1 \mathrm{~s}$ narrow scan of GO (Figure $8.3 \mathrm{c}$ ) can be fitted with two peaks related to carbonyl and carboxyl groups on the GO sheets, the O1s narrow scans of the GO coated Si-substrates reflect the additional presence of $\mathrm{SiO}_{2}$ and $\mathrm{MnO}_{2}$ (Figure 8.2 e and Figure S8.4). The appearance of the manganese peak can be traced back to the potassium permanganate customarily used during liquid synthesis of graphene oxide. During the electrophoretic coating process $\mathrm{MnO}_{2}$ can be formed, leading to the deposition of $\mathrm{MnO}_{2}$ nanoparticles (arrow in Figure 8.1 $\mathrm{c}$ and S8.1; see also the spikes in S8.11). A similar process has been discussed by $\mathrm{Yu}$ et al. for the synthesis of graphene $/ \mathrm{MnO}_{2}$ 
nanostructured textiles for improved capacitance in large-scale energy storage systems (Yu et al., 2011).

a

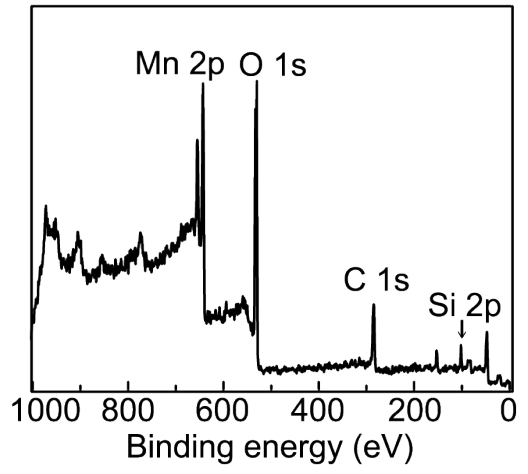

C

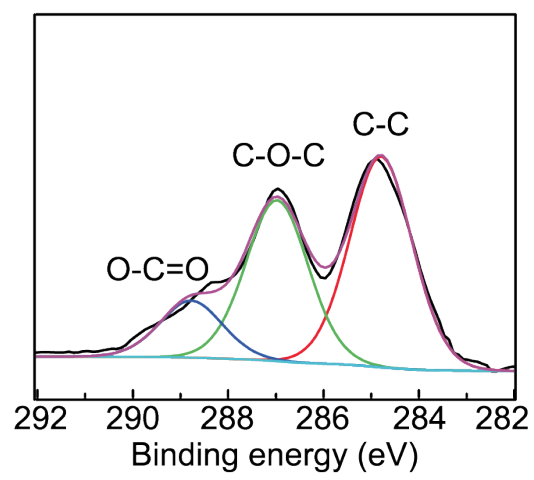

e

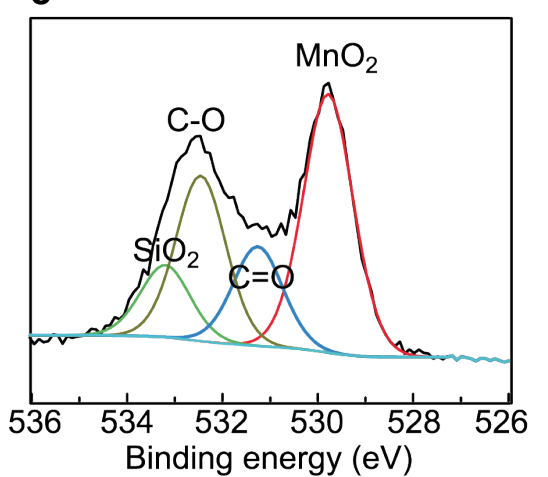

b

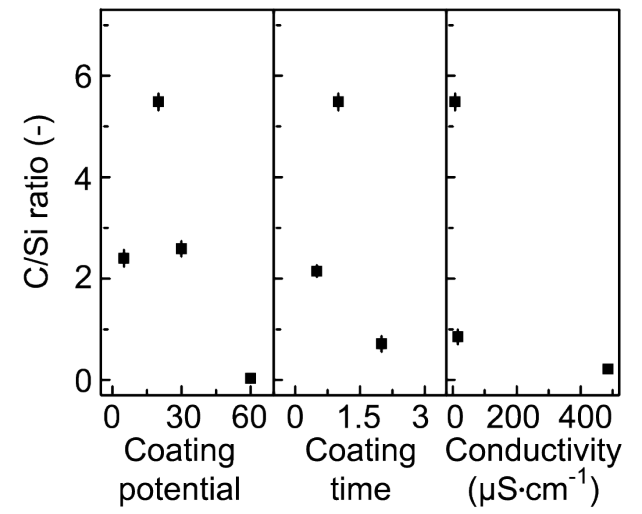

d

(V)

(h)

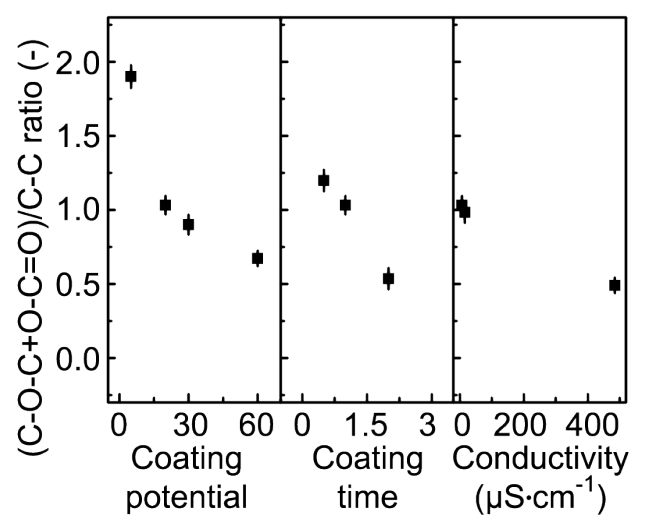

( $\mathrm{V})$

(h)

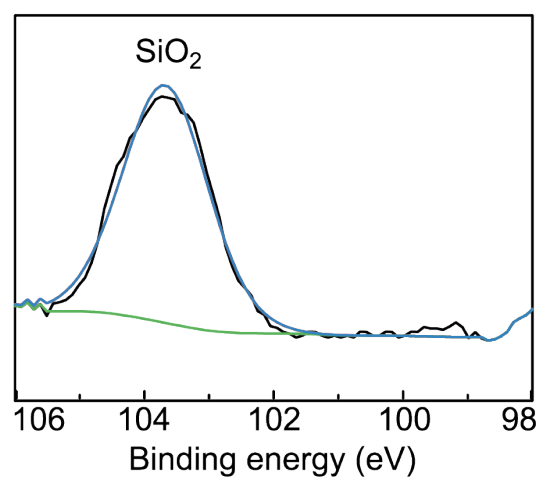

Figure 8.2. a XPS wide scan of a sample coated at $20 \mathrm{~V}$ for $1 \mathrm{~h}$ at $7 \mu \mathrm{S}^{\cdot} \mathrm{cm}^{-1}$. b Ratio of C/Si from XPS wide scans of samples coated at various potentials, coating times, and solution conductivities (standard deviations are $<10 \%$ ) c C1s XPS narrow scan of a sample coated at $20 \mathrm{~V}$ for $1 \mathrm{~h}$ at $7 \mu \mathrm{S}^{\cdot} \mathrm{cm}^{-1}$. d Ratio of O-bound carbon atoms versus $\mathrm{C}$-bound carbon atoms (standard deviations are $<10 \%$ ). O1s (e) and Si2p (f) XPS narrow scan of a sample coated at $20 \mathrm{~V}$ for $1 \mathrm{~h}$ at $7 \mu \mathrm{S}^{\cdot} \mathrm{cm}^{-1}$.

Looking at the XPS C1s narrow scans (Figure 8.2 c and S8.3) of the coated Si substrates the ratio of carbon atoms bound to oxygen atoms e.g. in carbonyl or 
carboxyl groups versus carbon atoms connected with only other carbon atoms decreases by $12-60 \%$ compared to the original ratio in $\mathrm{GO}$, indicating the electrochemical reduction of GO during the electrophoretic deposition process (Figure $8.2 \mathrm{~d}$ ) (An et al., 2010). Although the detailed mechanism behind this deoxygenation of carboxyl groups on the GO sheets is not the topic of our investigation, two literature-based hypotheses deserve mentioning, namely a Kolbe-like release of $\mathrm{CO}_{2}$ after contact with the electrode, leading to potential crosslinks between different GO sheets, (Diba et al., 2016; Vijh and Conway, 1967) or crosslinking between protic functional groups of $\mathrm{GO}$ and Si-containing molecules such as tetraethyl orthosilicates (Kou and Gao, 2011; Liu et al., 2015).

a

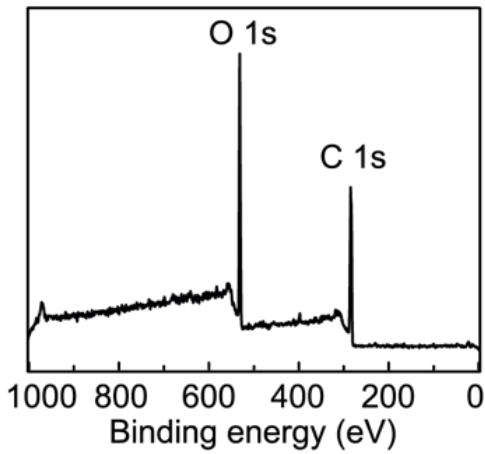

b

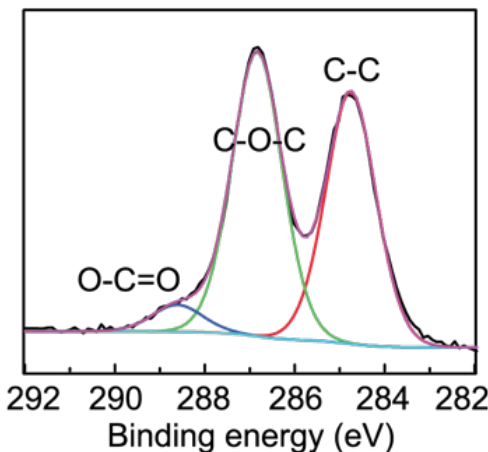

C

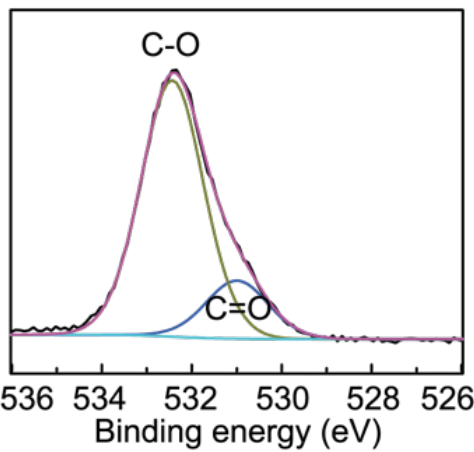

d

e
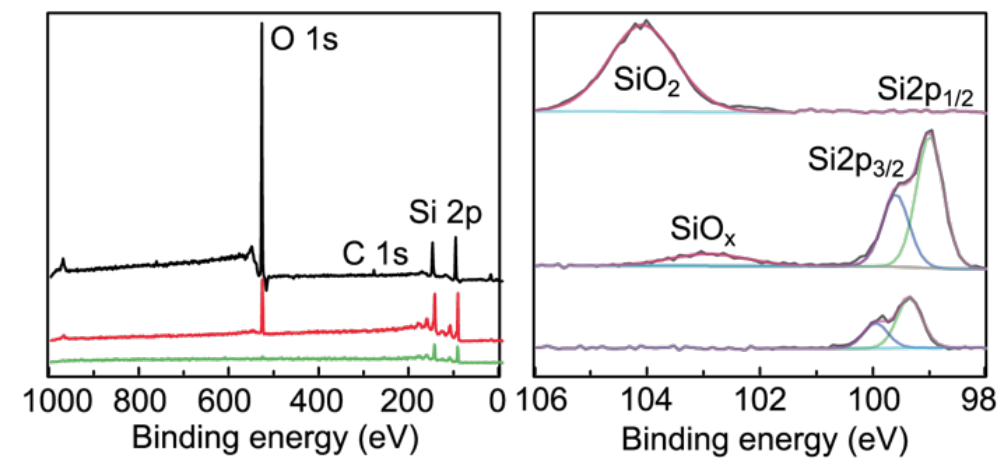

Figure 8.3. a XPS wide scan, $\mathbf{b}$ C1s narrow scan and $\mathbf{c}$ O1s narrow scan of GO. $\mathbf{d}$ XPS wide scan and e Narrow scan of HF-etched silicon substrate (bottom), silicon substrate exposed to $\mathrm{O}_{2}$ (middle), and thermally oxidized silicon substrate (top).

The samples coated at $30 \mathrm{~V}$ with the alternatively prepared $\mathrm{GO}(2)$ solution show similar results, the SEM image (Figure S8.7 a) reveals the presence of GO sheets on the surface and an O/C ratio of 5.6, as well as the shift of the Si peak to $103 \mathrm{eV}$ (Figure S8.7 c) indicative of the presence of silicon oxide. The absence of the Mn peak in the XPS spectra of $\mathrm{GO}(2)$ can be related to different ion residues in solution, similar 
to the samples coated with GO solution containing additional sodium sulfate (Figure \$8.2 c, d and Figure $\mathbf{8 8 . 4} \mathrm{c}$, d). This indicates that GO solution characteristics, such as the solution conductivity, are of high importance for the coating process and the resulting quality of the deposited layer.

a

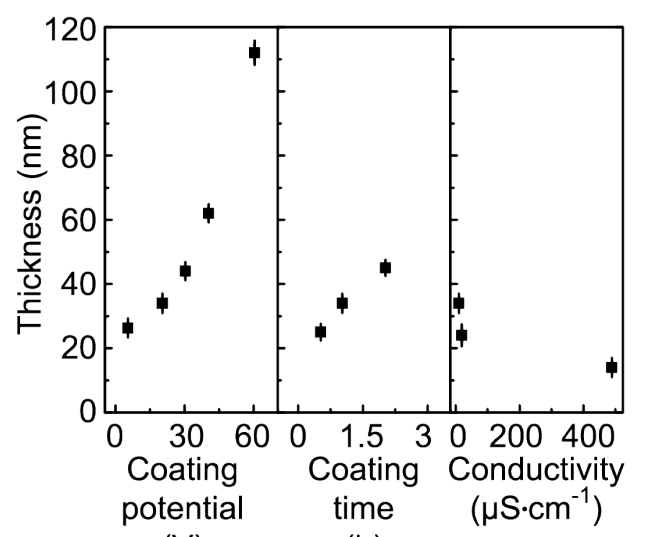

(V)

C

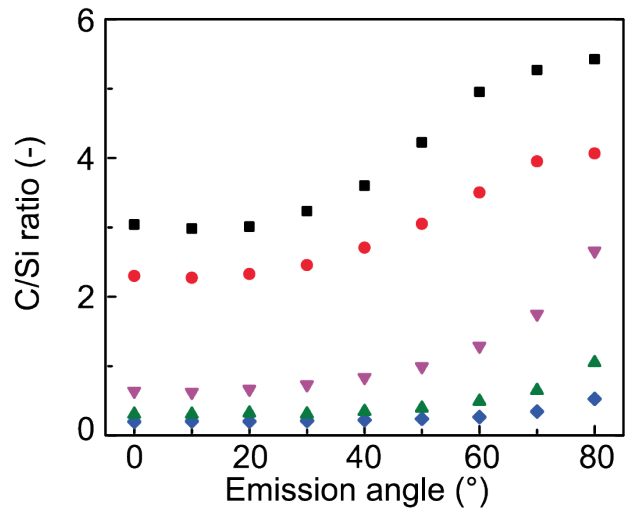

b

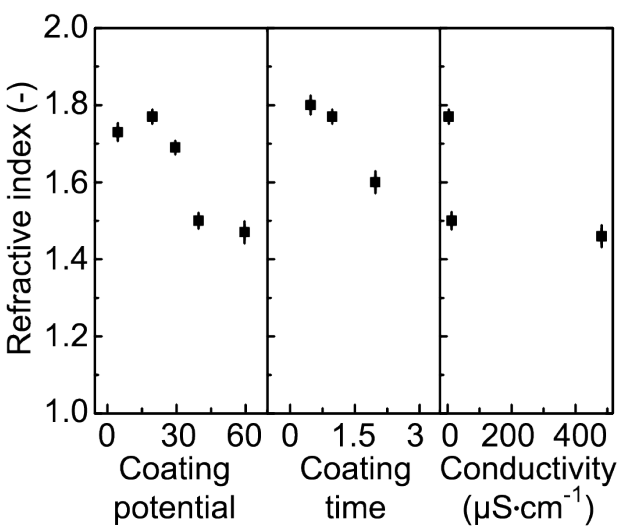

(V)

(h)

d

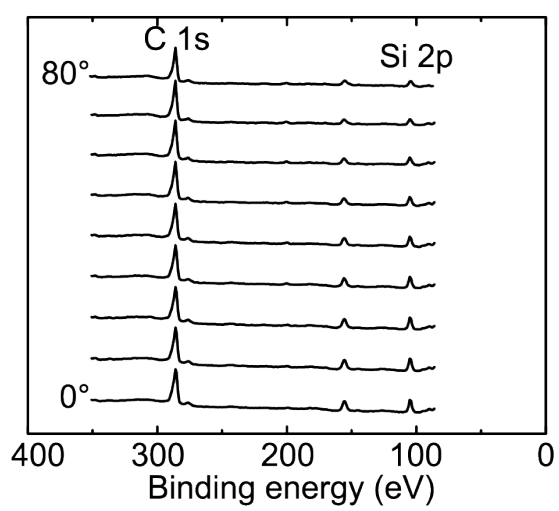

Figure 8.4. a Overall layer thickness ( $\mathrm{Si}$ oxide and $\mathrm{GO}$ ) and $\mathbf{b}$ Overall refractive index determined by ellipsometry (standard deviation is $<10 \%$ and $<2 \%$, respectively). c Ratio of $\mathrm{C}$ versus Si signal over XPS emission angle of samples coated at various potentials, coating times, and solution conductivities. Black square: $20 \mathrm{~V}, 1 \mathrm{~h}$, at $7 \mathrm{uS}{ }^{\cdot} \mathrm{cm}^{-1}$. Red circle: $30 \mathrm{~V}$, for $1 \mathrm{~h}$, at $7 \mu \mathrm{S}^{\cdot} \mathrm{cm}^{-1}$. Purple star: $40 \mathrm{~V}, 1 \mathrm{~h}$, at $7 \mu \mathrm{S}^{\cdot} \mathrm{cm}^{-1}$. Blue diamond: $60 \mathrm{~V}, 1 \mathrm{~h}$, at $7 \mu \mathrm{S}^{\circ} \mathrm{cm}^{-1}$. Pink downward triangle: $20 \mathrm{~V}, 1 \mathrm{~h}$, at $16 \mu \mathrm{S}^{\circ} \mathrm{cm}^{-1}$. Green upward triangle: $20 \mathrm{~V}, 1 \mathrm{~h}$ with, at $485 \mu \mathrm{S}^{\circ} \mathrm{cm}^{-1}$. Cross: $20 \mathrm{~V}, 0.5 \mathrm{~h}$, at $7 \mu \mathrm{S}^{\cdot} \mathrm{cm}^{-1}$. Star: $20 \mathrm{~V}, 2 \mathrm{~h}$, at $7 \mu \mathrm{S}^{\cdot} \mathrm{cm}^{-1}$. d XPS wide scans at different emission angles of a sample coated at $20 \mathrm{~V}$ for $1 \mathrm{~h}$ at a solution conductivity of $7 \mu \mathrm{S} \cdot \mathrm{cm}^{-1}$.

While no GO deposition and no silicon oxidation can be detected on a silicon substrate simply exposed to the GO solution without applying a potential (Figure S8.10), the overall thickness (silicon oxide and GO) of the layers formed when coated at $20 \mathrm{~V}$ for $1 \mathrm{~h}$ was $34 \mathrm{~nm}$, and height differences measured by AFM after applying a scratch in the GO layer (Figure S8.11), suggest the GO thickness to be 
around $15 \mathrm{~nm}$. With increasing potential the overall thickness increased to $112 \mathrm{~nm}$ (Figure 8.4 a), but the $\mathrm{C} / \mathrm{Si}$ ratio decreased as depicted in Figure $\mathbf{8 . 2} \mathbf{b}$.

The angle-resolved XPS images show a steeper rise of the C/Si ratio over the emission angles with higher coating potential (Figure $8.4 \mathrm{c}$ ) indicating that the carbon layer gets thinner, whereas the position of the Si2p peak is not changing (Figure $8.4 \mathbf{d}$ ). Furthermore, the refractive index shifts from 1.77 to 1.46 (Figure 8.4 b) at high coating potential, and as such becomes equal to the value of thermally oxidized silicon (1.46). This indicates that with increasing potential the amount of GO deposition is decreasing, which can be related to an increase in electrolysis of water and bubble formation at the interface, destroying the GO layer (Chávez-Valdez and Boccaccini, 2012; Diba et al., 2014). Furthermore due to the increased current, anodic silicon oxidation is favored (Zhang, 2001). This is supported by the cross sectional SEM images in Figure 8.5. On the $20 \mathrm{~V}$ sample the porous $\mathrm{GO}$ layer is visible, whereas at $60 \mathrm{~V}$ the layer appears flat and dense, similar to the sample coated with the reference $\mathrm{GO}(2)$ solution at $30 \mathrm{~V}$ (Figure S8.7 b).
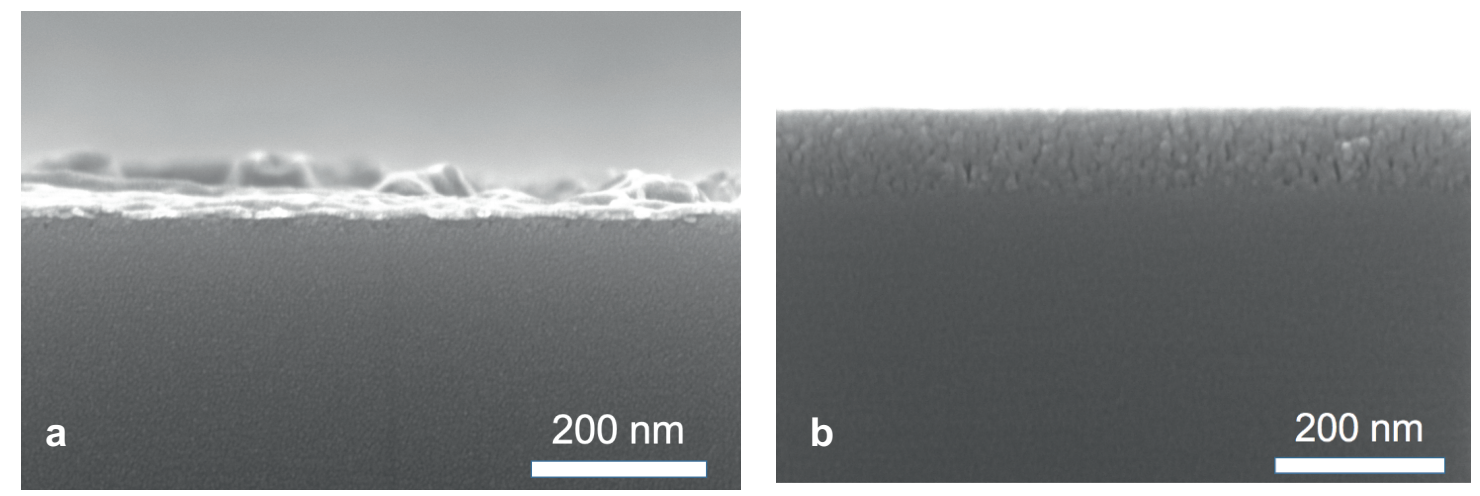

Figure 8.5. Cross sectional SEM images of samples coated at $\mathbf{a} 20 \mathrm{~V}$ and $\mathbf{b} 60 \mathrm{~V}$ for $1 \mathrm{~h}$ at a solution conductivity of $7 \mu \mathrm{S} \mathrm{cm}^{-1}$.

With increasing solution conductivity, both the C1s XPS peak and the overall thickness of the layers are decreasing, and the refractive index approaches 1.46 (i.e., again reaches the reference value for silicon oxide). This can be related to a stronger screening of the charges on the sheets and thus slower particle motion and less GO deposition at higher ionic strength (Besra and Liu, 2007; Ferrari and Moreno, 1997). At the same time as described before also a higher anion concentration leads to a higher ionic current, which can be related to more extensive surface reactions such as silicon oxidation (Zhang, 2001) and water electrolysis. The later causes bubble 
formation, and can thus lead to detachment of GO sheets (Chávez-Valdez and Boccaccini, 2012). A study of the layer composition as function of time shows that the C1s peak is first increasing (Figure S8.2 f) as is the overall layer thickness (Figure $\mathbf{8 . 4}$ a), which is then followed by a decrease in carbon signal (Figure $\mathbf{5 8 . 2} \mathbf{~ g}$ ) at a continued increase in overall layer thickness. This together with the decrease in refractive index towards that of silicon oxide (Figure $8.4 \mathrm{~b}$ ), leads us to conclude that first GO deposition takes place, while the GO thickness is later stagnating or even lost, while Si oxidation becomes the main factor for increasing overall thickness of the formed layer. This means that within one processing step a three-layered substrate (silicon / silicon oxide / graphene oxide) can be produced, and that the thickness of the different layers can be adjusted to some extent, depending on the coating time, coating potential and the conductivity of the solution.

\subsection{Conclusion}

During electrophoretic deposition of GO, away from deposition and anodic reduction of GO, also oxidation of the Si substrate takes place. This anodic oxidation of Si leads to color changes, and changes in the overall layer thickness. In literature, these effects were contributed to GO deposition, but here we show via SEM, XPS and AFM analyses the complex composition of the formed adlayer, in which both $\mathrm{GO}$ and SiOx appear in amounts and ratios dependent on deposition voltage, solution conductivity and reaction times. The more detailed analysis of the origin of this adlayer allows a better understanding of the use of GO layers in a wide variety of applications. 


\subsection{Supporting information}
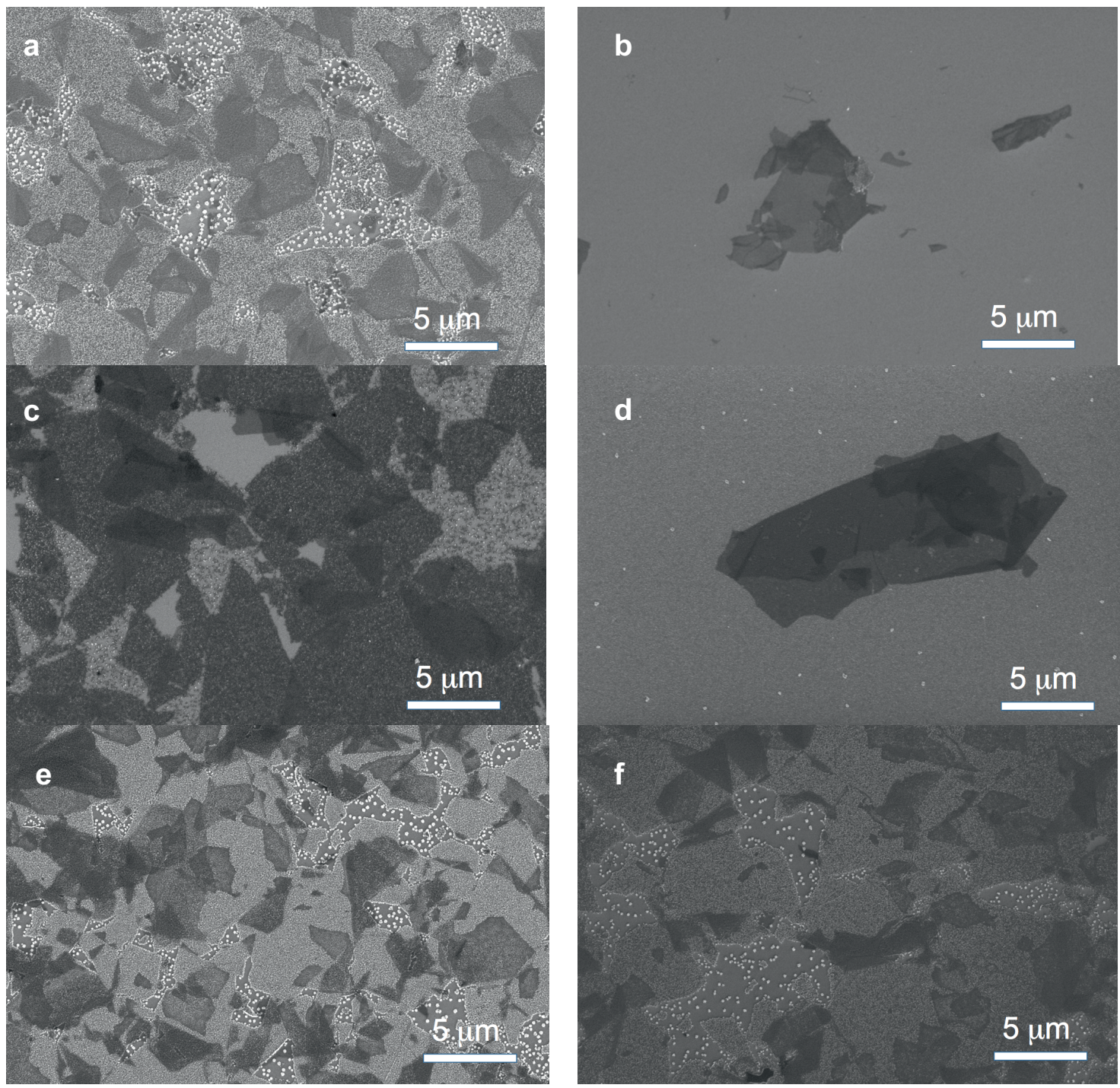

Figure S8.1. High magnification SEM images (10000 $\mathrm{x}$ ) of samples coated for $60 \mathrm{~min}$ at $\mathbf{a} 30$ and $\mathbf{b}$ $60 \mathrm{~V}$ at a solution conductivity of $7 \mu \mathrm{S}^{\cdot} \mathrm{cm}^{-1}$. In image $\mathbf{c}$ and $\mathbf{d}$, samples coated for $60 \mathrm{~min}$ at $20 \mathrm{~V}$ with a solution conductivity of 16 and $485 \mu \mathrm{S}^{\cdot} \mathrm{cm}^{-1}$, respectively. SEM images of samples coated at $20 \mathrm{~V}$ and $7 \mu \mathrm{S} \cdot \mathrm{cm}^{-1}$ for $30(\mathbf{e})$ and $120(\mathbf{f}) \mathrm{min}$ 
a

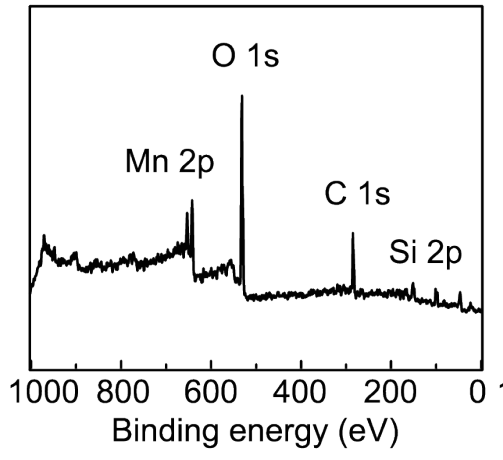

b

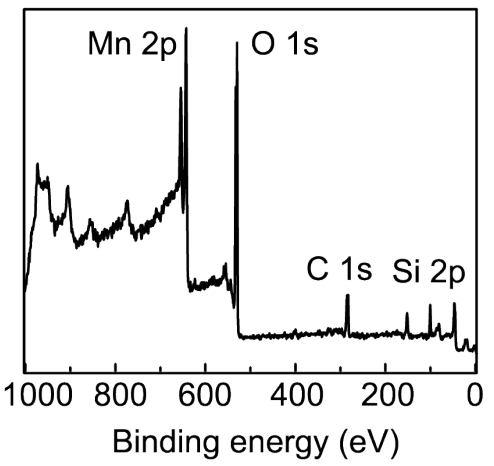

.

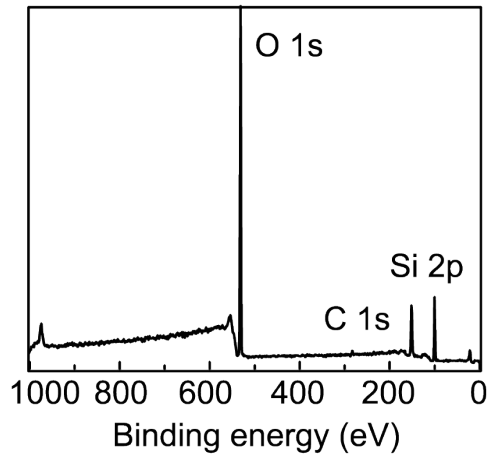

d

e

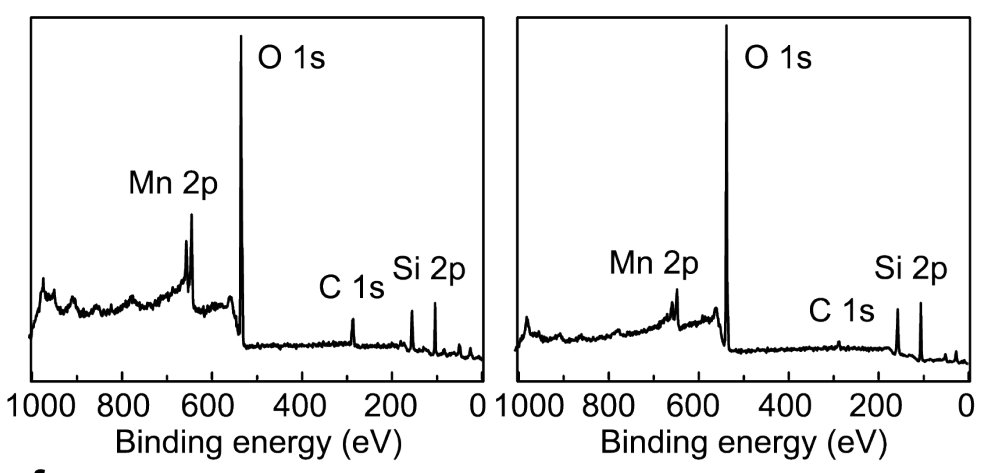

f

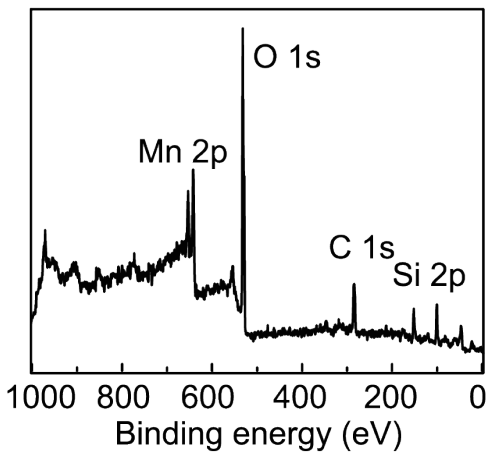

g

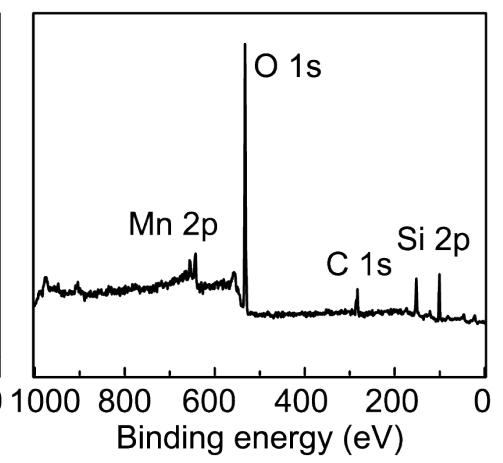

Figure S8.2. XPS wide scans of samples coated at 5,30 and $60 \mathrm{~V}$ for $1 \mathrm{~h}$, respectively (image $\mathbf{a}, \mathbf{b}$ and c) at a solution conductivity of $7 \mu \mathrm{S}^{\cdot} \mathrm{cm}^{-1}$. XPS of samples coated at $20 \mathrm{~V}$ for $1 \mathrm{~h}$ with a solution conductivity of 16 (d) and $485 \mu \mathrm{S}^{\circ} \mathrm{cm}^{-1}$ (e) and samples coated at $20 \mathrm{~V}$ at a solution conductivity of $7 \mu \mathrm{S} \cdot \mathrm{cm}^{-1}$ for $\mathrm{f} 30 \mathrm{~min}$ and $\mathrm{g} 120 \mathrm{~min}$. 
a

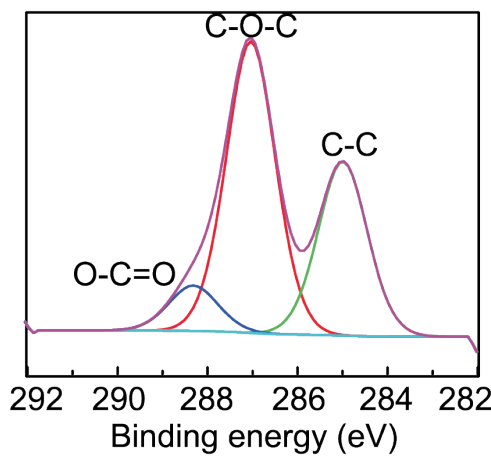

d

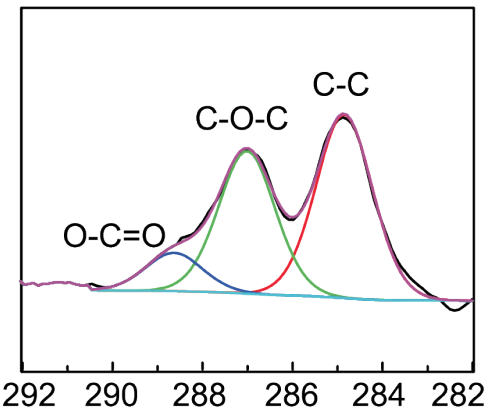

Binding energy (eV)

f

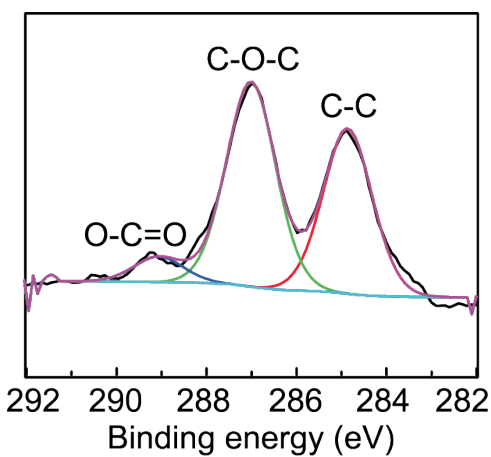

b

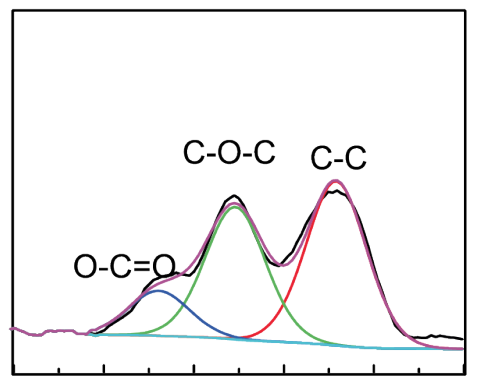

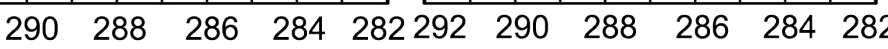

Binding energy (eV)
C

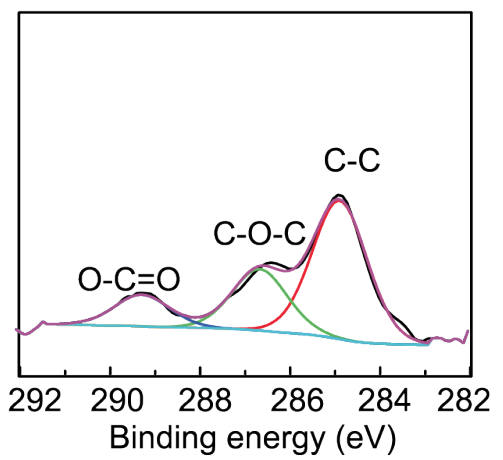

e

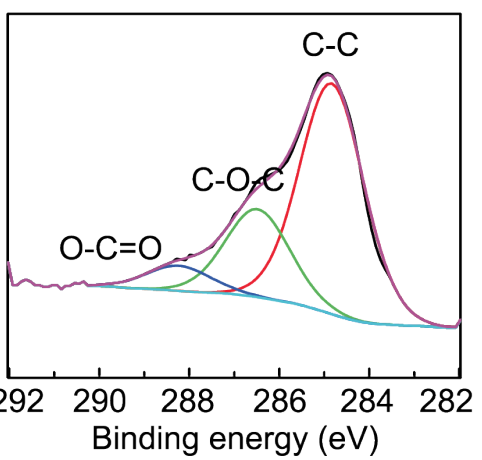

g

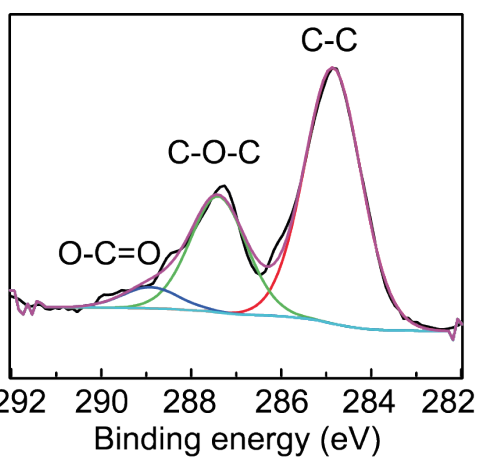

Figure S8.3. XPS C1s narrow scan of samples coated at 5,30 and $60 \mathrm{~V}$ for $1 \mathrm{~h}$, respectively (image a, b and c) at a solution conductivity of $7 \mu \mathrm{S}^{\cdot} \mathrm{cm}^{-1}$. XPS of samples coated at $20 \mathrm{~V}$ for $1 \mathrm{~h}$ with a solution conductivity of 16 (d) and $485 \mu \mathrm{S}^{\circ} \mathrm{cm}^{-1}$ (e) and samples coated at $20 \mathrm{~V}$ at a solution conductivity of $7 \mu \mathrm{S} \cdot \mathrm{cm}^{-1}$ for $\mathbf{f} 30 \mathrm{~min}$ and $\mathbf{g} 120 \mathrm{~min}$. 
a

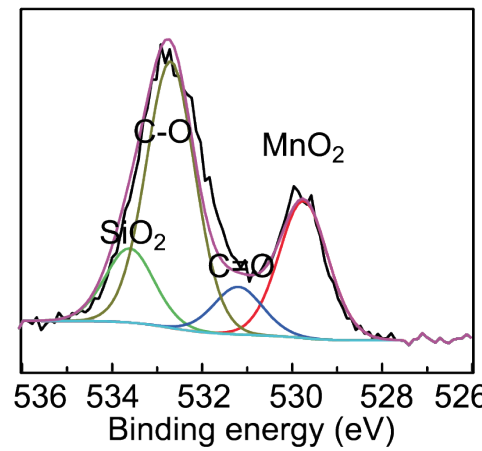

Binding energy (eV) b

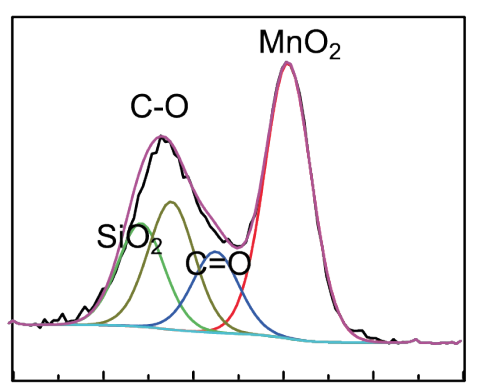

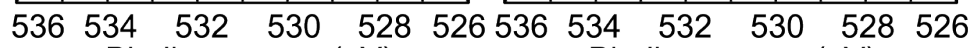
Binding energy $(\mathrm{eV}) \quad$ Binding energy $(\mathrm{eV})$
C

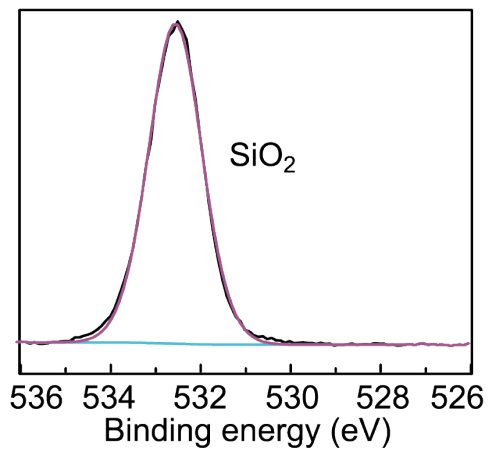

d

e
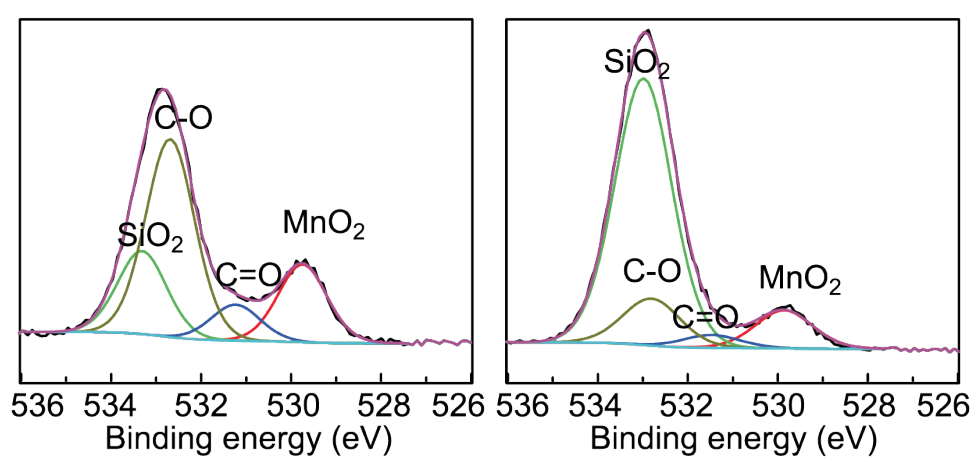

$\mathbf{f}$

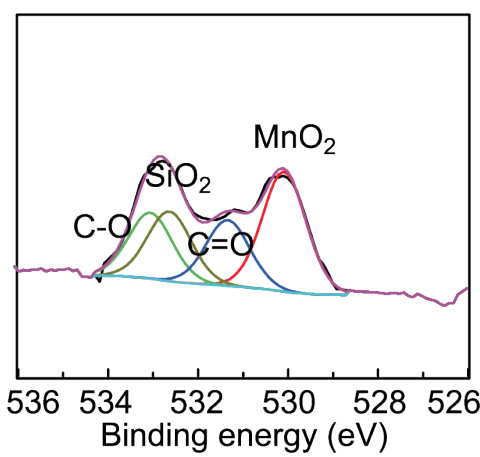

g

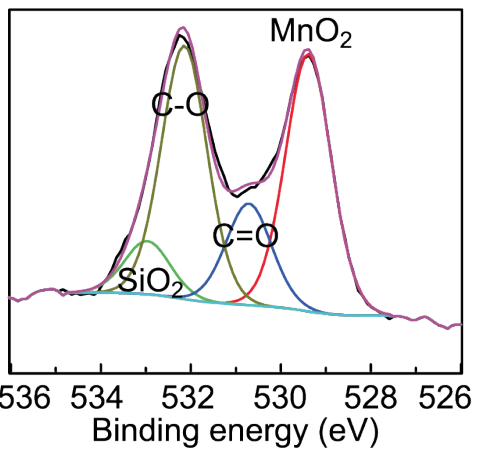

Figure S8.4. XPS O1s narrow scan of samples coated at 5, 30 and $60 \mathrm{~V}$ for $1 \mathrm{~h}$, respectively (image a, b and c) at a solution conductivity of $7 \mu \mathrm{S}^{\cdot} \mathrm{cm}^{-1}$. XPS of samples coated at $20 \mathrm{~V}$ for $1 \mathrm{~h}$ with a solution conductivity of $16(\mathbf{d})$ and $485 \mu \mathrm{S}^{\cdot} \mathrm{cm}^{-1}(\mathbf{e})$ and samples coated at $20 \mathrm{~V}$ at a solution conductivity of $7 \mu \mathrm{S}^{\cdot} \mathrm{cm}^{-1}$ for $\mathrm{f} 30 \mathrm{~min}$ and $\mathrm{g} 120 \mathrm{~min}$. 
a

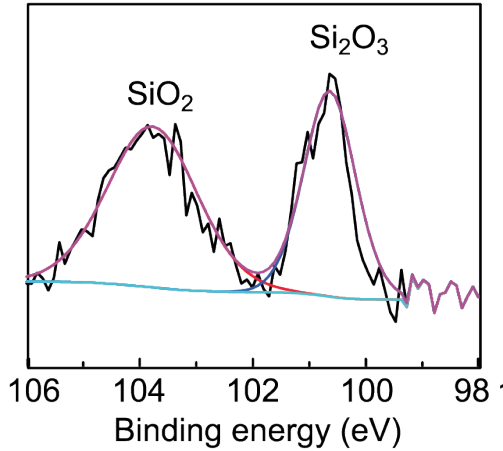

d

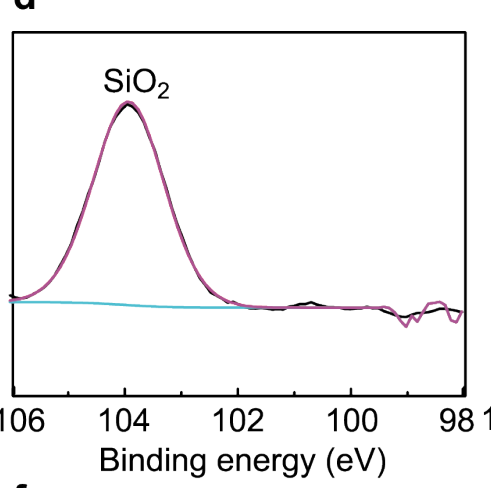

f

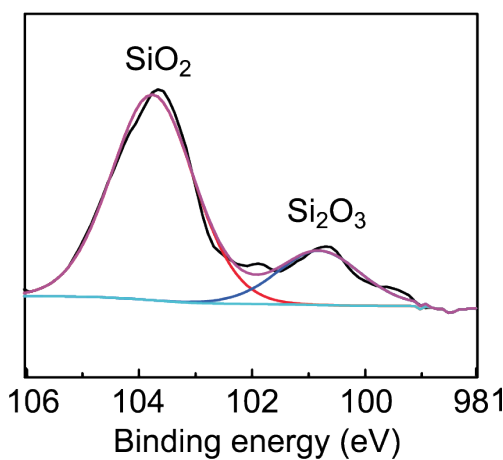

b

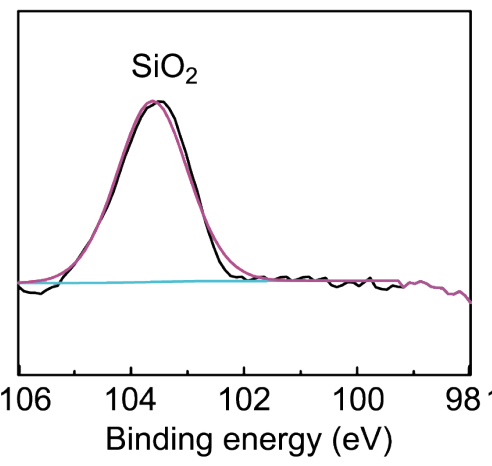

e

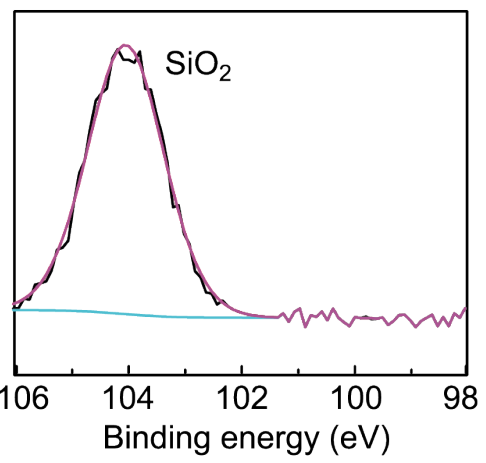

g

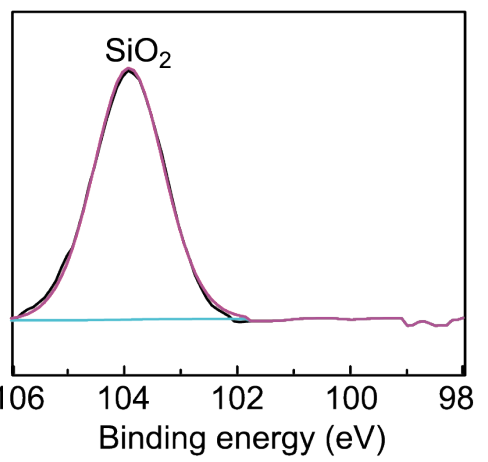

Figure S8.5. XPS Si2p narrow scan of samples coated at 5,30 and $60 \mathrm{~V}$ for $1 \mathrm{~h}$, respectively (image a, b and c) at a solution conductivity of $7 \mu \mathrm{S}^{\cdot} \mathrm{cm}^{-1}$. XPS of samples coated at $20 \mathrm{~V}$ for $1 \mathrm{~h}$ with a solution conductivity of 16 (d) and $485 \mu \mathrm{S}^{\cdot} \mathrm{cm}^{-1}(\mathbf{e})$ and samples coated at $20 \mathrm{~V}$ at a solution conductivity of $7 \mu \mathrm{S} \cdot \mathrm{cm}^{-1}$ for $\mathbf{f} 30 \mathrm{~min}$ and $\mathrm{g} 120 \mathrm{~min}$. 

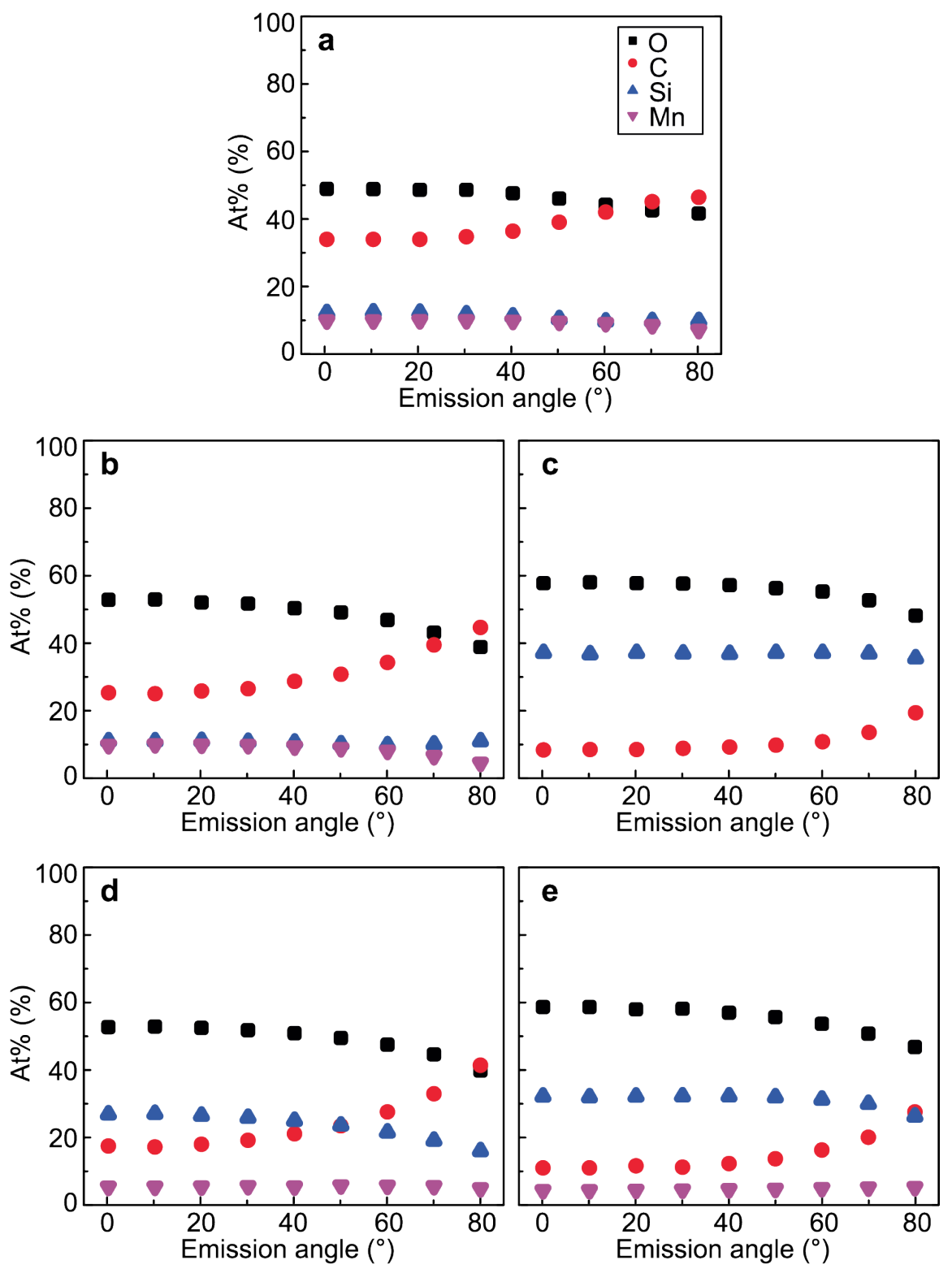

Figure S8.6. Angle-resolved XPS results (a-c) of samples coated at 20,30 and $60 \mathrm{~V}$ for $1 \mathrm{~h}$ respectively, at a solution conductivity of $7 \mu \mathrm{S}^{\cdot} \mathrm{cm}^{-1}$ and samples coated at $20 \mathrm{~V}$ for $1 \mathrm{~h}$ with a solution conductivity of d 16 and e $485 \mu \mathrm{S} \cdot \mathrm{cm}^{-1}$. 
a

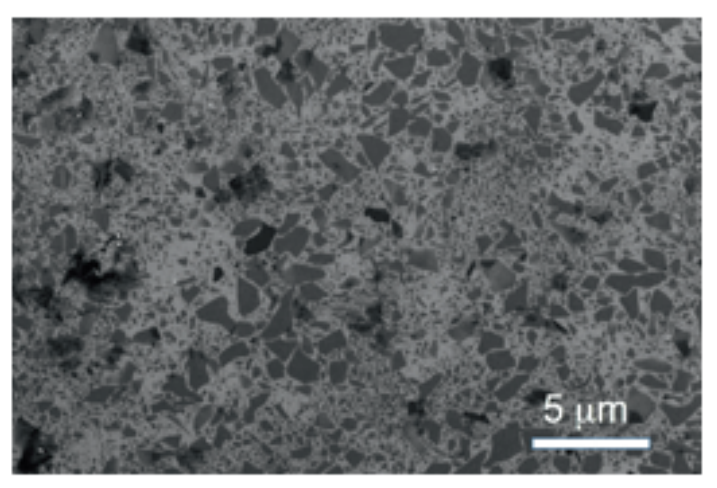

b
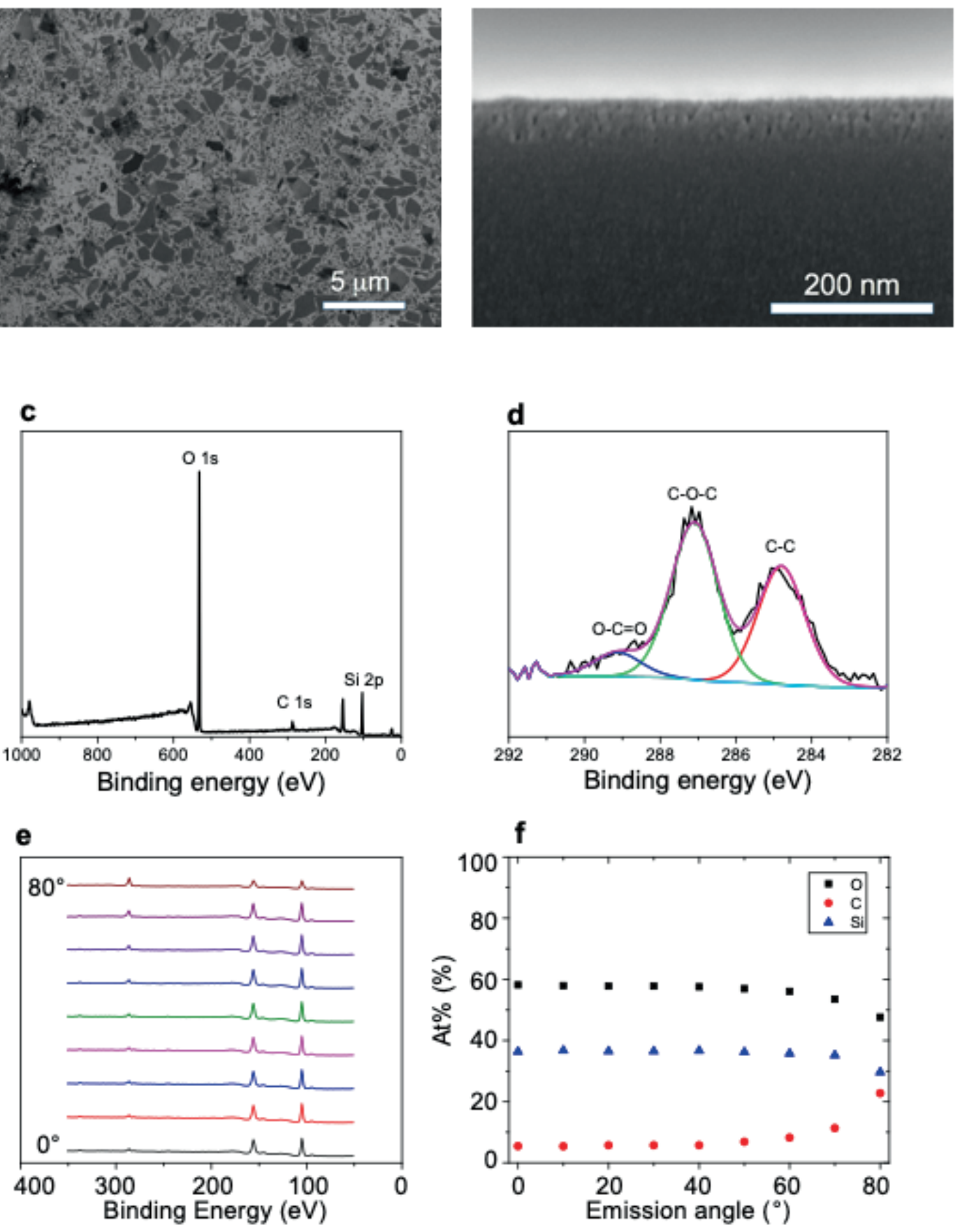
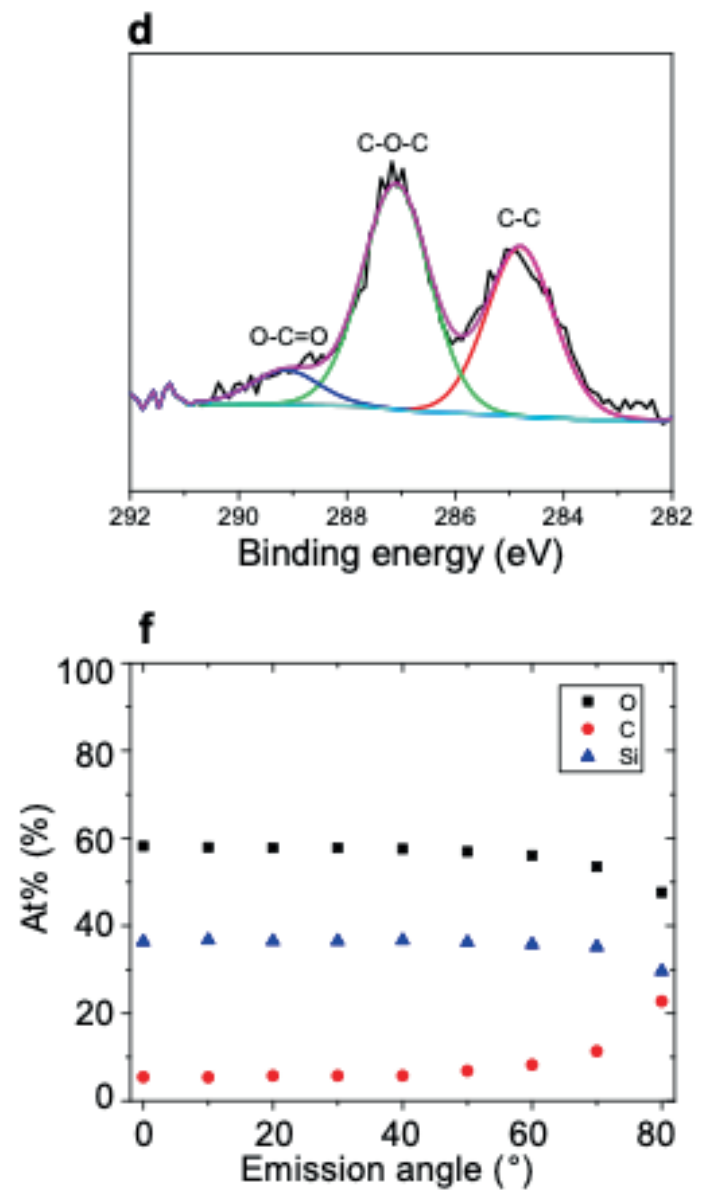

Figure S8.7. SEM image of the top (a) and the cross section (b) of a silicon substrate coated with GO2 solution at $30 \mathrm{~V}$ for $1 \mathrm{~h}$. XPS wide scan (c), C1s narrow scan (d) and angle resolved XPS spectra of the sample described in a. $\mathbf{f}$ Change in atomic $\%$ of the $\mathrm{C} 1 \mathrm{~s}$, O1s and Si2p peak with varying XPS emission angle. 


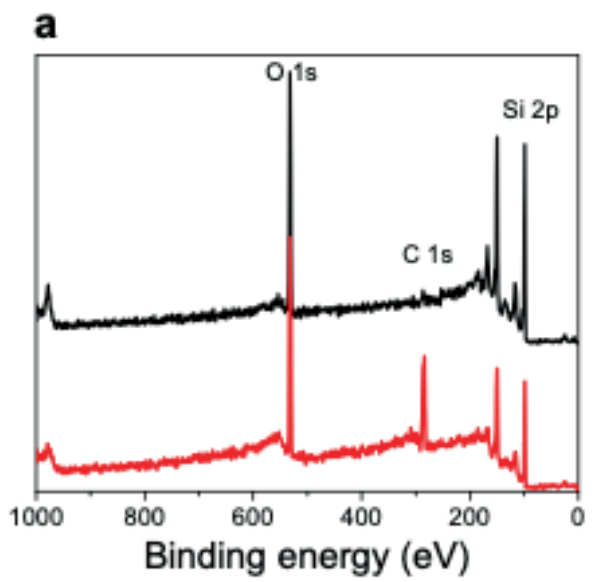

b

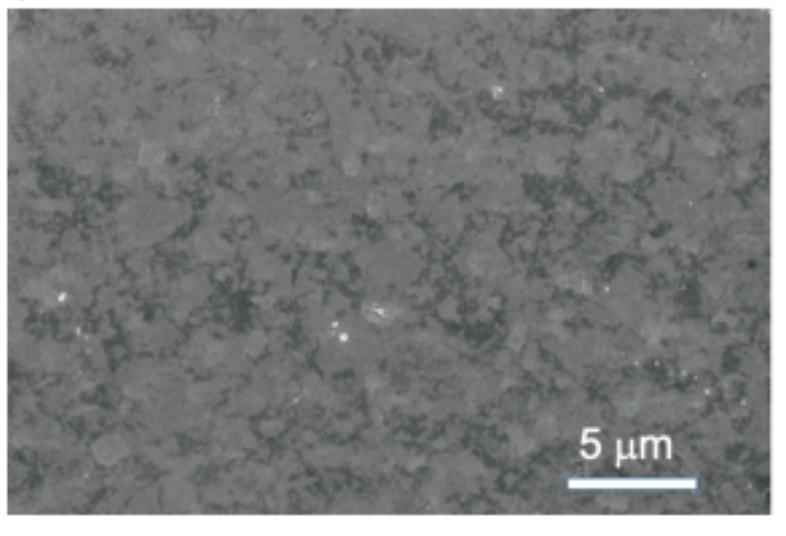

Figure S8.8. Si substrates spin coated with GO2 solution. a XPS wide scan of $\mathrm{Si}$ substrate spin coated with (red) and without (black) previous etching with HF. b SEM image of sample spin coated after etching with HF.
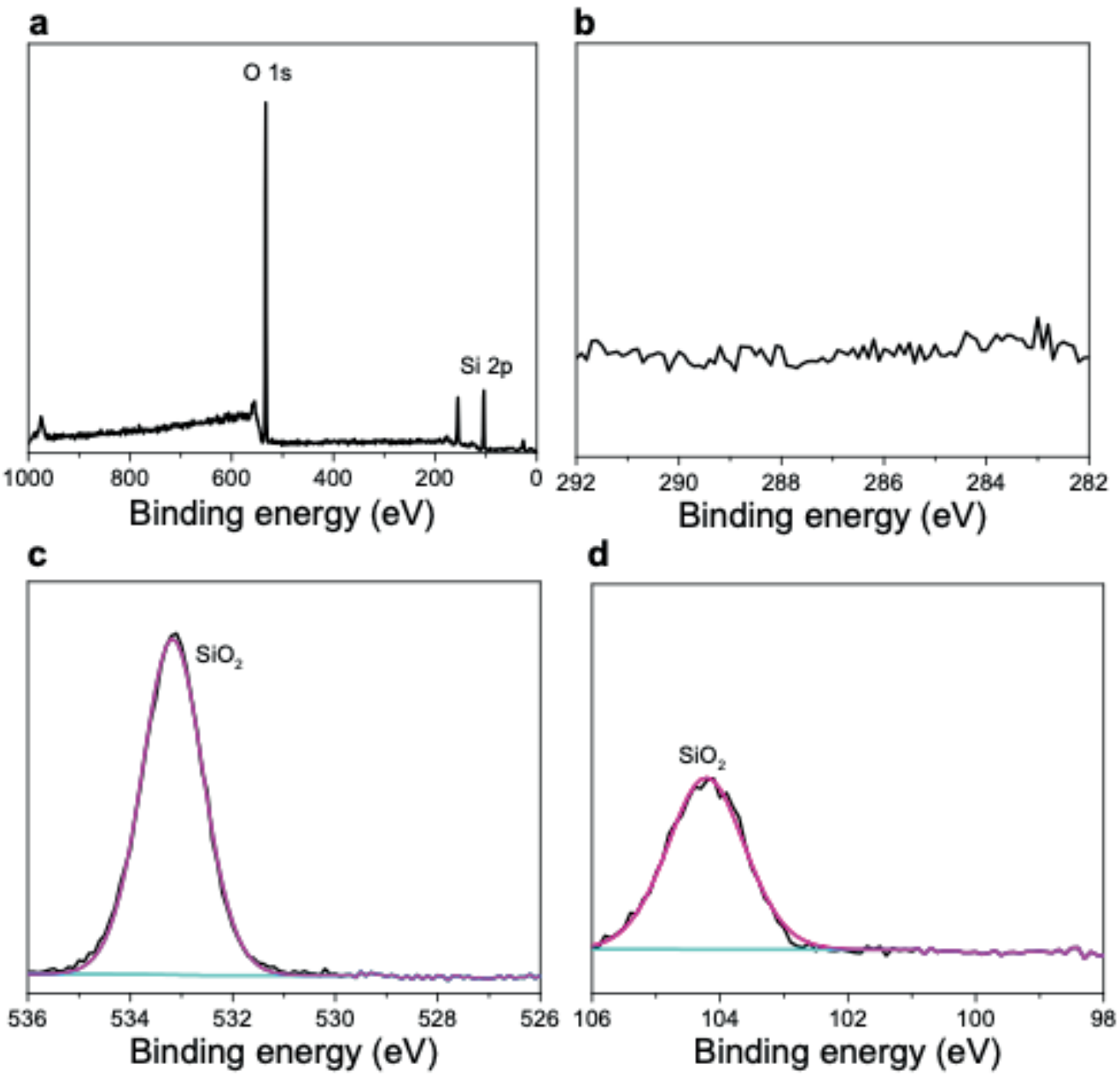

Figure S8.9. XPS spectra of Si substrates exposed to $20 \mathrm{~V}$ for $1 \mathrm{~h}$ in $7 \mu \mathrm{S}^{\cdot} \mathrm{cm}^{-1}$ sodium sulfate. a Wide scan, b C1s narrow scan, $\mathbf{c}$ O1s narrow scan, $\mathbf{d}$ Si2p narrow scan. 

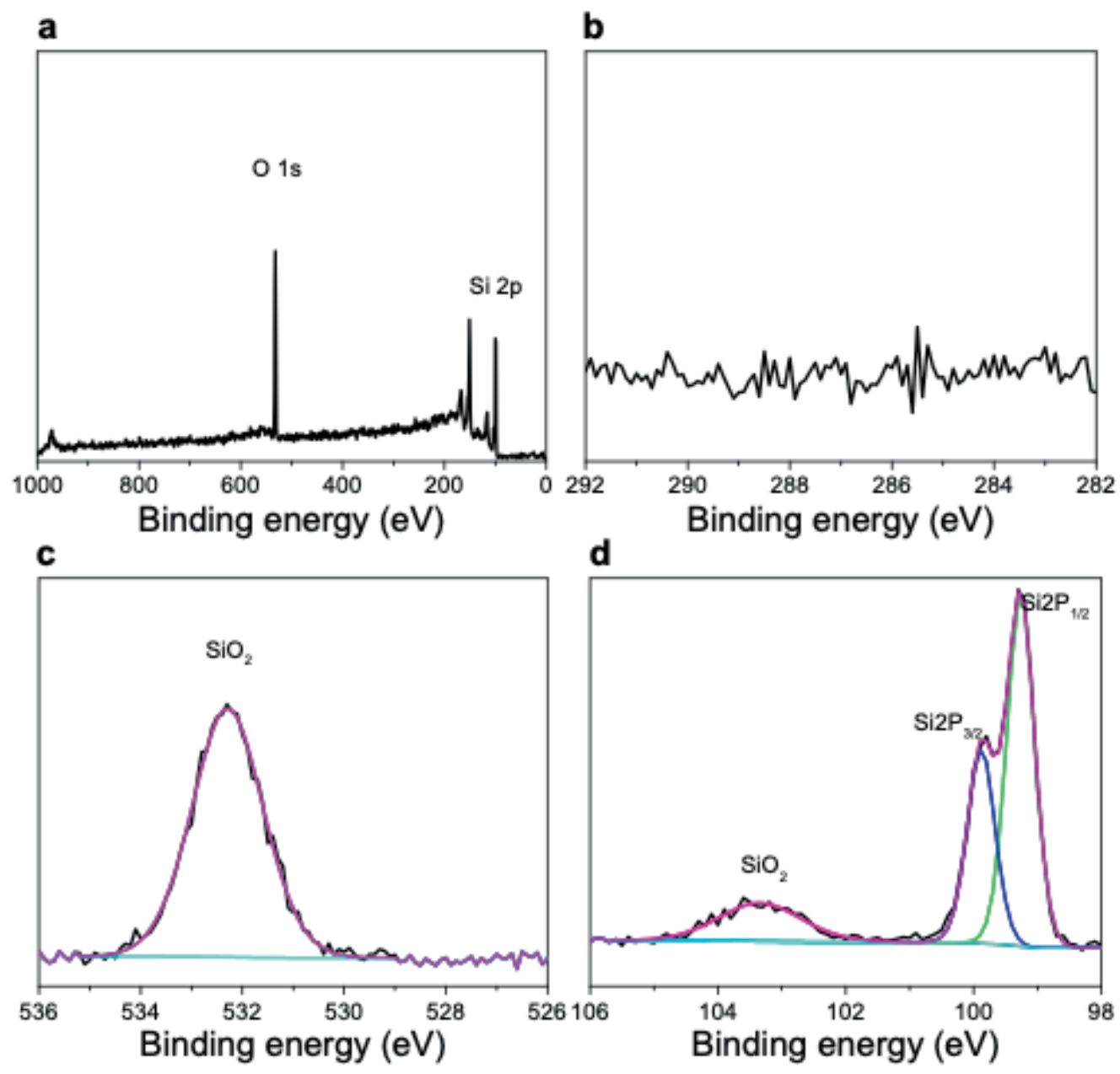

Figure S8.10. XPS spectra of Si substrates exposed to GO solution for $1 \mathrm{~h}$ without applied potential. a wide scan, b C1s narrow scan, c O1s narrow scan, d Si2p narrow scan.
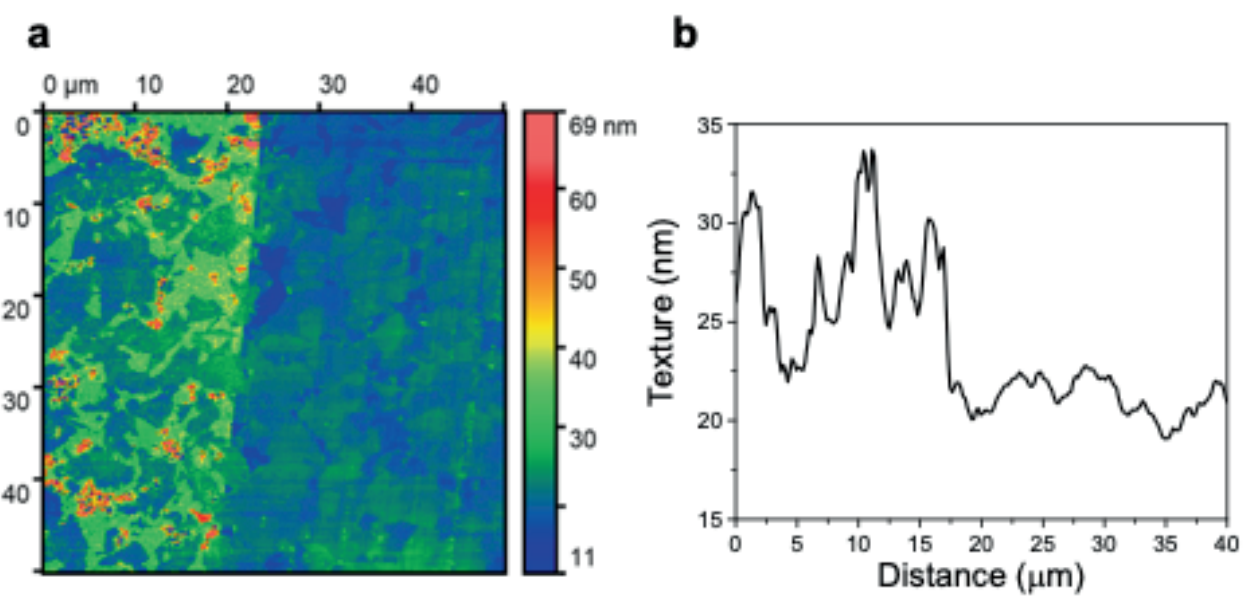

Figure S8.11. AFM image of Si substrate coated with $\mathrm{GO}$ at $20 \mathrm{~V}$ for $1 \mathrm{~h}$ and $7 \mu \mathrm{S}^{\circ} \mathrm{cm}^{-1}$ indicating height differences on the sample. a Top view b Texture. 



\section{CHAPTER 9}

\section{GENERAL DISCUSSION}




\section{GENERAL DISCUSSION}

The aim of this thesis was to explore and extend the use of electrochemical principles that are currently mostly applied for salt removal, and selective isolation or fractionation of proteins. This included the identification and quantification of the underlying phenomena, and establishing design rules for innovative processes that make use of these principles, ideally applied to practical situations. In doing so, the environmental impact of the currently used chromatographic separation processes may be mitigated by avoiding the extensive use of solvents or buffers. Furthermore, electrochemical separation processes have the potential to switch very fast between different states e.g. from negative to positive surface charge or high to low surface wetting, which will allow size reduction of the current separation installations and thus process costs. Electrochemical separation of macromolecules is still in its early stages of development, and the thesis aims to bring this technology to a next level.

\subsection{Main findings in perspective}

As described in Chapter 2, electrochemical stimuli have been used mainly for the separation of low molecular weight ions whereas for proteins and cells electrochemical separation solutions are still scarce. A prominent process within the class of electrochemical ion separation processes is capacitive deionization, which stores ions in electric double layers by biasing the electrodes (Suss et al., 2015; Yoon et al., 2019). Some strategies exist to render the process more selective towards a specific type of ion by considering the difference in ion size and shape, resulting in different adsorption rates (Seo et al., 2010; Zhao et al., 2012b); however, the selectivity is rather low, and especially difficult to maintain when working with complex streams that may result in separation loss for example due to fouling of the electrodes. This becomes even more prominent when aiming at electrochemical protein separation and selectively targeting one protein solely via an electric field. Inverted capacitive deionization is considered a better starting point for electrochemical protein separation, since the target molecules adsorb spontaneously due to specific interactions with the electrode surface that even may be modified to prevent undesired interactions, and desorb only due to an applied electric bias (Gao et al., 2015a). 
Chapters 3 and 4 focus on the separation of ions by inverted capacitive deionization using conventional activated carbon electrodes and polyelectrolyteactivated carbon composite electrodes. These studies show that this process can be performed either by adding polyelectrolytes into the activated carbon electrode matrix or by mounting ion exchange membranes in front of it. When polyelectrolyte-activated carbon composite electrodes are used in combination with ion exchange membranes, the performance is even competitive with conventional membrane capacitive deionization using the same carbon material, and shows very low exergy losses. The process may be further improved by the use of materials with more micropores to increase the amount of stored ions (Suss et al., 2015); however, this would diverge from the components of interest of this thesis, and thus this was no longer pursued.

When aiming at the electrochemical separation of proteins, protein migration to and from the electrode interface needs to be controlled and irreversible adsorption avoided. To approach this challenge we analyze in Chapter 5 to 8 , as well as in some additional data discussed below, how protein attraction and repulsion to and from the electrode interface can be impacted by the surface chemistry, and most importantly how this can be altered by the applied potential. For this we investigate salt and protein ad- and desorption in electrochemical flow cells and analyze at fundamental level how the applied potential influences electrostatic and hydration interactions, key players in protein-surface interactions.

In Chapter 5 we show that a zwitterionic coating on a carbon electrode strongly decreases irreversible protein adsorption. This is also illustrated in Figure 9.1, were we study protein ad- and desorption to and from gold-chips that are modified with zwitterionic coatings in a surface plasmon resonance (SPR) device. The SPR allows to monitor the amount of protein directly at the interface and gives thus additional information about the events occurring at the electrode. Figure $9.1 \mathrm{c}$ shows that for thick and dense polymer layers (blue), hardly any $\beta$-lactoglobulin adsorption occurs and that this is not influenced by the potential applied. This is useful if the aim is to decrease electrode fouling and thus increase their life time in desalination processes as discussed in Chapter 5.

If the zwitterionic layer is however only very thin up to $0.8 \mathrm{ng} / \mathrm{mm}^{2}$, protein adsorbs on the coated gold electrodes and some desorption is detected at a positive potential, because the protein is positively charged at $\mathrm{pH} 4$. This indicates that in principle by varying the thickness and density of the anti-fouling coatings, suitable 
electrodes for controlled protein ad- and desorption can be made, although the protein adsorption is in this case still below monolayer coverage, which is at $2-3 \mathrm{ng} / \mathrm{mm}^{2}$.

a

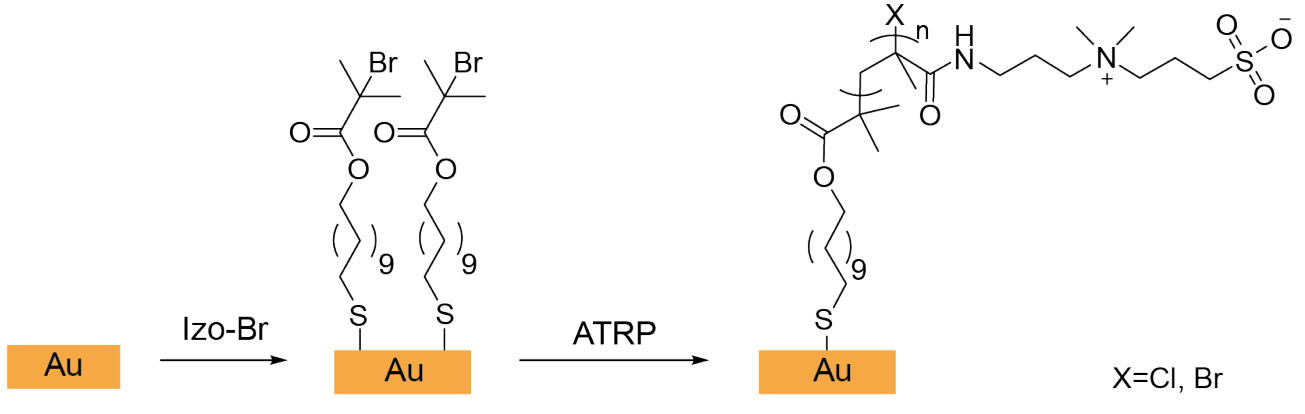

b

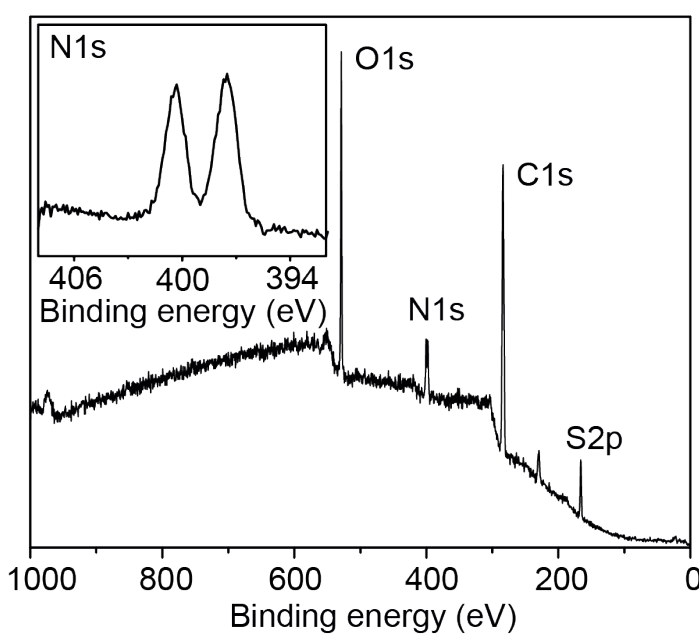

C

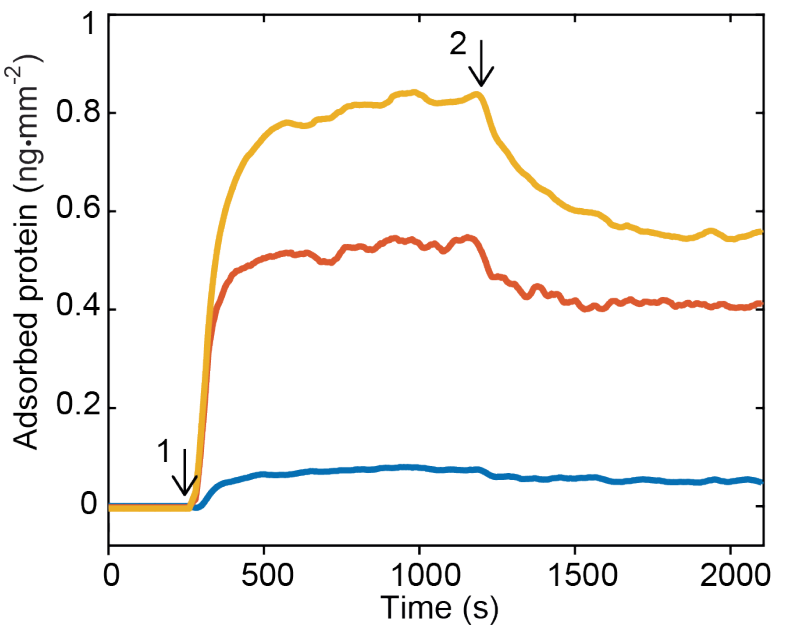

Figure 9.1. $\beta$-lactoglobulin adsorption to zwitterionic coatings. a Reaction scheme of ATRP initiator application (Izo-Br) on SPR-gold-chip and subsequent ATRP to grow zwitterionic brushes made of sulfobetadine monomers. The reaction conditions can be found elsewhere (Lange et al., 2016). b X-ray photoelectron spectroscopy wide scan of SPR-gold chip after ATRP and N1s narrow scan (insertion). c $\beta$-lactoglobulin adsorption to SPR-gold chip with zwitterionic brush of different length and initiator dilution (Izo-Br:Octanethiol). Blue: $10.1 \mathrm{~nm}$ and 1:10, Red: $2.7 \mathrm{~nm}$ and 1:10, Yellow: $3.4 \mathrm{~nm}$ and 1:20. The brush length was measured with ellipsometry. The protein was dissolved in $10 \mathrm{mM}$ phosphate buffer at $\mathrm{pH} 6$ and exposed to the surface after $300 \mathrm{~s}$ (arrow 1). After $900 \mathrm{~s}$ (arrow 2) the surface was rinsed with buffer and exposed to a potential of $0.2 \mathrm{~V}$.

By adding polyelectrolytes on the SPR-gold-chip surface, spontaneous protein adsorption can be increased as shown in Figure $\mathbf{9 . 2}$ for an anionic polymer, while protein desorption is still possible by applying a potential. This is in line with Chapter 6 , where polyelectrolyte-activated carbon composite electrodes were used (the same as in Chapter 4), or alternatively dip coated activated carbon electrodes. When following the concentration of proteins and ions in the outlet of the electrochemical flow cell in Chapter 6, we see, that at $0 \mathrm{~V}$ applied, proteins adsorb and salt desorbs, whereas at $-1.2 \mathrm{~V}$, salt adsorbs and proteins desorb. This indicates an ion exchange process which 
is further supported by the capacitive ion storage and protein release. The dip coated electrodes show the highest protein and salt adsorption per gram polymer used; their salt yield being comparable to standard activated carbon electrodes. This makes these electrodes interesting candidates for use in processes aimed at subsequent removal of salt and protein, or for enrichment of specific proteins as measured for $\beta$-lactoglobulin. Especially the latter is interesting for example for the dairy industry, to prepare fractions rich in $\beta$-lactoglobulin that are known to be highly effective in foam and emulsion formation.

a

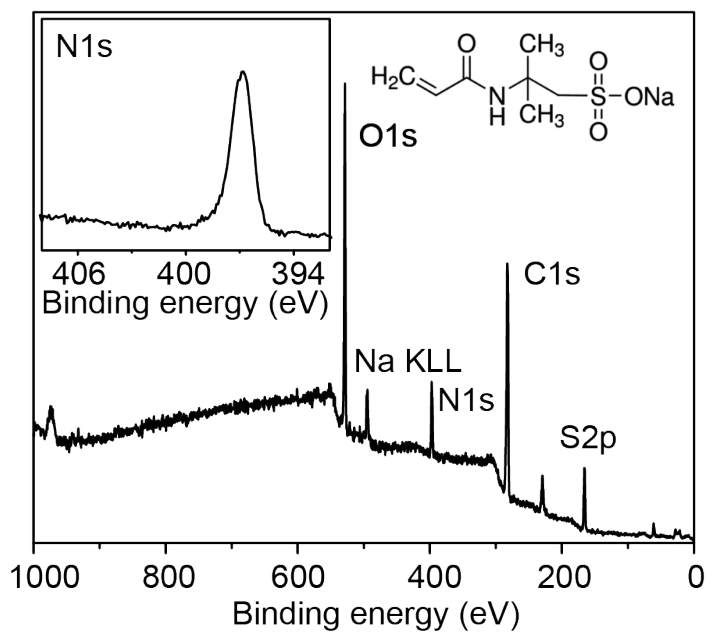

b

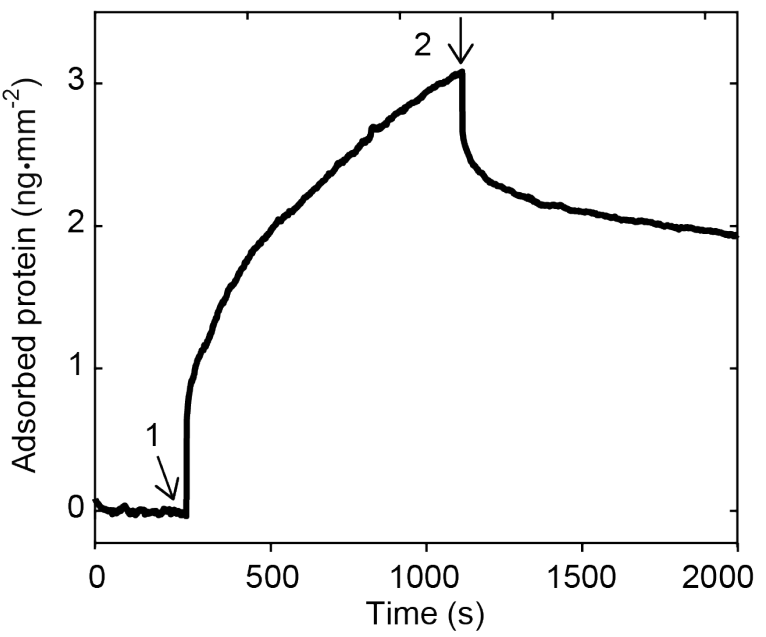

Figure 9.2. $\beta$-lactoglobulin adsorption on anionic coating. a X-ray photoelectron spectroscopy wide scan and N1s narrow scan (insertion left) of SPR gold chip modified with 2-acrylamido-2-methyl-1propanesulfonate (insertion right) following the same reaction scheme as shown in Figure 1a. b $\beta$-lactoglobulin adsorption to SPR gold chip with 2-acrylamido-2-methyl-1-propanesulfonate brush of thickness $0.6 \mathrm{~nm}$ and initiator dilution (Izo-Br:Octanethiol) 1:100. $\beta$-lactoglobulin was dissolved in $5 \mathrm{mM}$ sodium chloride at pH 6 and exposed to the surface after $300 \mathrm{~s}$ (arrow 1). After $900 \mathrm{~s}$ (arrow 2) the surface was rinsed with buffer and exposed to a potential of $0.6 \mathrm{~V}$.

In Chapter 7, we describe in depth the underlying mechanisms as studied using model surfaces. The applied potential to a gold electrode does not only influence the electrostatic interactions but it also changes the wetting of the surface which is related to the hydration repulsion. This indicates that the electrowetting properties of the electrodes should be addressed by the design criterion for electrochemical protein separation processes. Since these measurements could only be conducted on a gold substrate, in Chapter 8 a method is proposed to coat silicon wafers with graphene oxide sheets, which makes these surfaces suitable for reflectometric studies, allowing 
similar studies on carbon-based materials, which will support the design of novel electrodes.

\subsection{Process design and outlook}

In comparison to chromatography and filtration, electrochemical separation has the advantage that it can be used to target both protein and salt separation separately or combined, depending on the electrode properties (Table 9.1). While reversed osmosis filtration has developed into a mature process to desalinate water, even at high salt concentration, it requires high energy inputs. For protein separation, filtration can only be used for fractionation, resulting in products of relatively low purity, since separation by size is not specific. Furthermore, membrane fouling is a major drawback when filtering protein-rich streams. In contrast, chromatography is known for protein products of very high purity, but due to the high costs, this process is rarely used for desalination or to produce protein fractions. In addition to that, this method requires large amounts of solvents or buffers.

With filtration and chromatography, separation of salt and protein within one processing step is not possible, making this a unique feature of electrochemical separation processes. At the same time, electrochemical separation can be used for only salt separation (e.g. when coating the electrodes with zwitterionic electrodes (Chapter 5)) or only protein separation (e.g. when adding a large amount of polyelectrolytes into the matrix of the electrodes (Chapter 6)). Inherent to electrochemical process is that solvent use is zero, while protein purities may be high (although not demonstrated in this thesis), since the adsorption step still depends on molecule specific surface interactions. This points out the versatility of this promising and seminal technology.

Nevertheless, the advantages of electrochemical separation processes need to be weighed against its resource efficiency, and have to be concretized into a full life cycle analysis and compared to conventional adsorptive separation and filtration processes. This should be combined with the separation performance and cost of the electrodes, module and peripheral system, which allows further development of the separation, therewith improving the competitiveness relative to current separation technologies. 
Table 9.1. Comparing applications, energy and solvent use as well as processing cost for filtration, chromatography and electrochemical separation. Note: the symbol + stands for high target suitability, high energy and solvent use as well as high cost.

\begin{tabular}{|c|c|c|c|c|c|c|c|}
\hline \multirow{2}{*}{$\begin{array}{l}\text { Separation } \\
\text { method }\end{array}$} & \multicolumn{4}{|c|}{ Target } & \multirow{2}{*}{$\begin{array}{c}\text { Energy } \\
\text { use }\end{array}$} & \multirow{2}{*}{$\begin{array}{c}\text { Solvent } \\
\text { use }\end{array}$} & \multirow[t]{2}{*}{ Cost } \\
\hline & Salt & $\begin{array}{c}\text { Protein } \\
\text { (pure) }\end{array}$ & $\begin{array}{l}\text { Protein } \\
\text { (fraction) }\end{array}$ & $\begin{array}{c}\text { Salt }+ \\
\text { Protein }\end{array}$ & & & \\
\hline Filtration & ++ & - & ++ & - & ++ & - & + \\
\hline Chromatography & - & ++ & + & - & + & ++ & ++ \\
\hline $\begin{array}{l}\text { Electrochemical } \\
\text { separation }\end{array}$ & ++ & + & ++ & ++ & + & - & + \\
\hline
\end{tabular}

Key points that should be addressed to bring the technology further are the design criteria for the electrodes and respective coatings depending on the target molecule as summarized in Table 9.2, as well as for process upscaling (Table 9.3). For salt removal, increasing the number of nanopores increases the ion separation capacity, and should be strived for. However, protein separation has different requirements due to the larger molecular size of the proteins and their tendency to clog pores. Furthermore, the use of antifouling coatings is beneficial for adsorptive removal of salt from complex mixtures. If for example protein contaminants are present in the saline water, an antifouling coating prevents protein adsorption and thus increases the lifetime of the electrodes, as shown in Chapter 5. Charged coatings may be used for inverted or enhanced capacitive deionization although compared to nanopores the effect that can be created is less relevant. However, when considering selective electrochemical protein separation, the use of charged polymers is essential since this largely increases the protein separation capacity and makes it selective, without making the electrode preparation more complex. Also depending on the location of the polymer simultaneous salt separation is possible as shown in Chapter 6.

Table 9.2. Design criteria for salt and protein separation

\begin{tabular}{lcccc}
\hline Design criteria & & Target & & $\begin{array}{c}\text { Complexity of } \\
\text { preparation }\end{array}$ \\
\cline { 2 - 4 } & Salt & Protein & Salt + protein & ++ \\
of nanopores & ++ & - & + & + \\
\hline Antifouling coatings & ++ & - & ++ & + \\
\hline $\begin{array}{l}\text { Charged } \\
\text { coatings }\end{array}$ & + & ++ & + & ++ \\
\hdashline $\begin{array}{l}\text { Responsive } \\
\text { coatings }\end{array}$ & - & ++ & + & + \\
\hline
\end{tabular}


As a next step, electro-responsive materials/coatings such as mercaptohexadecanoic acid and ferrocene (as discussed in Chapter 2) are promising for protein separation, in spite of their complexity of preparation. It is expected that both types of coatings will allow protein separation at lower potentials, and imporve the surface regeneration, leading to even more stable long-term operation.

The technology of electro-responsive coatings is however still in full development and depending on the target molecule and aim of the separation, different properties can be chosen. Potential developments for protein separation could be using tailored block-copolymers that contain zwitterionic and cationic or anionic parts. In Figure 9.3, examples of cationic-zwitterionic block-copolymer coatings and their functioning are shown. When a positive potential is applied, the cationic polymer is repelled from the electrode, and forms positively charged anchoring points within the zwitterionic part of the layer. When exposed to a solution, negatively charged proteins bind to the islands, while the rest of the electrode is protected by the antifouling properties of the zwitterionic portion of the coating. If the potential is reversed, the cationic polymer is attracted towards the electrode surface and is no longer reachable for the protein - thus the repulsive properties of the zwitterionic blocks dominate, leading to repellence of the previously bound proteins.

These coatings may be prepared using established polymerization techniques such as addition-fragmentation chain transfer polymerization (RAFT) or initiated ATRP. Furthermore, irreversible protein adsorption may be avoided and improved control over ad- and desorption might be achieved.

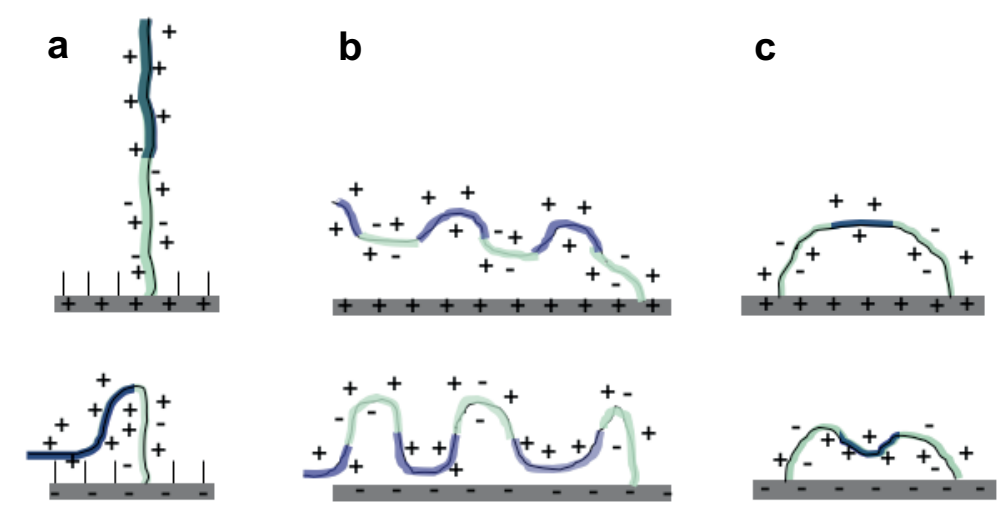

Figure 9.3. Schematic of electro-responsive block-copolymers. a block copolymer with a zwitter ionic and a cationic part attached with one end to the electrode surface. b multi block copolymer attached with one end to the electrode surface and $\mathbf{c}$ block co polymer attached with both ends to the electrode surface. 
Next to that, in order to make electrochemical (protein) separation practically relevant, the separation capacity needs to be increased. In capacitive deionization the most conventional set up consists of two parallel electrodes with the target solution flowing in between these two electrodes, although also column systems exist, such as used in electrochemical modulated liquid chromatography (Harnisch and Porter, 2001).

In upscaling electrochemical reactors, it is very important to ensure homogeneity in electric current or potential, just as homogeneity of geometry, kinematics and thermal conditions are important in thermo-chemical reactors (Sulaymon, A. H.Abbar, 2012). A homogeneous electric current and/or potential throughout the system is ensured by a constant electrode gap. Scaling to larger volumes and capacities van be achieved by englarging the surface area of the individual electrodes while keeping the electrode gap constant (Walsh and Ponce de León, 2018). As mentioned before for salt separation, this can be achieved via three dimensional electrodes such as made from porous carbon materials (Suss et al., 2015). An additional approach often chosen for upscaling of flow-by capacitive deionization, is stacking of electrode pairs (Welgemoed and Schutte, 2005) which is possible in various ways (see also Table 9.3). For cost-effective, truly large scale application for separations with intermediate added value as in the food sector, a plate configuration may be too capital intensive, and spiral wound configurations should be preferred, as customary in membrane separation, especially nanofiltration and reverse osmosis (Table 9.3).

Table 9.3. Comparing stack, column and spiral wound configurations for up-scaling. Note: the symbol + stands for large electrode size, good electrode packing as well as high flow rate and costs.

\begin{tabular}{lccccc}
\hline Configuration & Electrode size & Electrode packing & Flow rate & Cost \\
\hline Stack & ++ & + & ++ & ++ \\
\hdashline Spiral wound & ++ & ++ & ++ & - \\
\hdashline Column & + & - & + & ++ \\
\hline
\end{tabular}

\subsection{Conclusion}

The work described in this thesis showed that ion as well as protein separation is possible with porous carbon electrodes modified with polyelectrolytes while using electrochemical stimuli. This is the first step towards a new generation of adsorptive separation processes that are more resource efficient, have a strongly reduced environmental impact and are economically feasible. To drive this ambition further 
research is required to build on our findings and optimize surface chemistry, electrode composition, and process design. 

REFERENCES 
Alexander, P., Brown, C., Arneth, A., Finnigan, J., Moran, D., and Rounsevell, M.D.A. (2017). Losses, inefficiencies and waste in the global food system. Agric. Syst. 153, 190-200.

Ali, I. (2014). Water treatment by adsorption columns: evaluation at ground level. Sep. Purif. Rev. 43, 175-205.

Ameli, A., and Alizadeh, N. (2011). Nanostructured conducting molecularly imprinted polymer for selective extraction of salicylate from urine and serum samples by electrochemically controlled solidphase micro-extraction. Anal. Chim. Acta 707, 62-68.

Ameli, A., and Alizadeh, N. (2012). Nanostructured conducting molecularly imprinted polymer for selective uptake/release of naproxen by the electrochemically controlled sorbent. Anal. Biochem. 428, 99-106.

An, S.J., Zhu, Y., Lee, S.H., Stoller, M.D., Emilsson, T., Park, S., Velamakanni, A., An, J., and Ruoff, R.S. (2010). Thin film fabrication and simultaneous anodic reduction of deposited graphene oxide platelets by electrophoretic deposition. J. Phys. Chem. Lett. 1, 1259-1263.

Anderson, M.A., Cudero, A.L., and Palma, J. (2010). Capacitive deionization as an electrochemical means of saving energy and delivering clean water. Comparison to present desalination practices: Will it compete? Electrochim. Acta 55, 3845-3856.

Arai, T., and Norde, W. (1990). The behavior of some model proteins at solid-liquid interfaces 1 . Adsorption from single protein solutions. Colloids and Surfaces 51, 1-15.

Arca, M., Mirkin, M. V, and Bard, A.J. (1995). Polymer Films on Electrodes. 26. Study of ion transport and electron transfer at polypyrrole films by scanning electrochemical microscopy. J.

Phys. Chem. 99, 5040-5050.

Arcila-Velez, M.R., and Roberts, M.E. (2014). Redox solute doped polypyrrole for high-charge capacity polymer electrodes. Chem. Mater. 26, 1601-1607.

Asghar, A., Anjum, F.M., and Allen, J.C. (2011). Utilization of dairy byproduct proteins, surfactants, and enzymes in frozen dough. Crit. Rev. Food Sci. Nutr. 51, 374-382.

Aslan, M., Zeiger, M., Jäckel, N., Grobelsek, I., Weingarth, D., and Presser, V. (2016). Improved capacitive deionization performance of mixed hydrophobic/hydrophilic activated carbon electrodes. J. Phys. Condens. Matter 28, 114003.

Balasubramanian, M., Giacomini, M.T., Lee, H.S., McBreen, J., and Sukamto, J.H. (2002). X-ray absorption studies of poly(vinylferrocene) polymers for anion separation. J. Electrochem. Soc. 149, D137.

Balogun, M., Qiu, W., Yang, H., Fan, W., Huang, Y., Fang, P., Li, G., Ji, H., and Tong, Y. (2016). A monolithic metal-free electrocatalyst for oxygen evolution reaction and overall water splitting. Energy Environ. Sci. 9, 3411-3416.

Bard, A.J., and Faulkner, L.R. (1944). Electrochemical methods - fundamentals and applications (John Wiley \& Sons, Inc.).

Barten, D., Kleijn, J.M., Duval, J., Leeuwen, H.P. V, Lyklema, J., and Cohen Stuart, M.A. (2003a). Double layer of a gold electrode probed by AFM force measurements. Langmuir 19, 1133-1139.

Barten, D., Kleijn, J.M., and Cohen Stuart, M.A. (2003b). Adsorption of a linear polyelectrolyte on a gold electrode. Phys. Chem. Chem. Phys. 5, 4258. 
Beaglehole, D., Webster, B., and Werner, S. (1998). Ellipsometry study of the adsorption of molecules at electrolyte interfaces with gold and stainless steel. J. Colloid Interface Sci. 202, 541550.

Becerril, H.A., Mao, J., Liu, Z., Stoltenberg, R.M., Bao, Z., and Chen, Y. (2008). Evaluation of Solution-Processed Reduced Graphene Oxide Films as Transparent Conductors. ACS Nano 2, 463470.

Beer, P.D., and Bayly, S.R. (2005). Anion Sensing by Metal-Based Receptors. In Anion Sensing, (Springer Berlin Heidelberg), pp. 125-162.

Besra, L., and Liu, M. (2007). A review on fundamentals and applications of electrophoretic deposition (EPD). Prog. Mater. Sci. 52, 1-61.

Besseling, N.A.M. (1997). Theory of Hydration Forces between Surfaces. Langmuir 13, 2113-2122.

Bi, H., Liu, Z., Xu, F., Tang, Y., Lin, T., and Huang, F. (2016a). Three-dimensional porous graphenelike carbon cloth from cotton as a free-standing lithium-ion battery anode. J. Mater. Chem. A 4, 11762-11767.

Bi, H., Lin, T., Xu, F., Tang, Y., Liu, Z., and Huang, F. (2016b). New Graphene Form of Nanoporous Monolith for Excellent Energy Storage. Nano Lett. 16, 349-354.

Biesheuvel, P.M. (2015). Activated carbon is an electron-conducting amphoteric ion adsorbent. 1-9. Biesheuvel, P.M., and Dykstra, J.E. (2018). The difference between Faradaic and Nonfaradaic processes in Electrochemistry. 1-10.

Biesheuvel, P.M., and van der Wal, A. (2010). Membrane capacitive deionization. J. Memb. Sci. 346, 256-262.

Biesheuvel, P.M., Zhao, R., Porada, S., and van der Wal, A. (2011). Theory of membrane capacitive deionization including the effect of the electrode pore space. J. Colloid Interface Sci. 360, 239-248.

Biesheuvel, P.M., Porada, S., Levi, M., and Bazant, M.Z. (2014). Attractive forces in microporous carbon electrodes for capacitive deionization. J. Solid State Electrochem. 18, 1365-1376.

Biesheuvel, P.M., Hamelers, H.V.M., and Suss, M.E. (2015). Theory of Water Desalination by Porous Electrodes with Immobile Chemical Charge. Colloids Interface Sci. Commun. 9, 1-5.

Blaedel, W.J., and Strohl, J.H. (1965). Partition Chromatography by Electrodeposition in a Mercury Film. Anal. Chem. 37, 64-67.

Bonomo, R., Minim, L., Coimbra, J., Fontan, R., Mendes da Silva, L., and Minim, V. (2006).

Hydrophobic interaction adsorption of whey proteins: Effect of temperature and salt concentration and thermodynamic analysis. J. Chromatogr. B 844, 6-14.

Bos, M.A., Shervani, Z., Anusiem, A.C.I., Giesbers, M., Norde, W., and Kleijn, J.M. (1994). Influence of the electric potential of the interface on the adsorption of proteins. Colloids Surfaces $B$ Biointerfaces 3, 91-100.

Breadmore, M.C. (2007). Recent advances in enhancing the sensitivity of electrophoresis and electrochromatography in capillaries and microchips. Electrophoresis 28, 254-281.

Bruckenstein, S., Fensore, A.T., and Hillman, A.R. (1998). Time-Resolved Mono-anion, Di-anion, and Solvent Transfers into a Poly(vinylferrocene)-Modified Electrode. J. Electrochem. Soc. 145, 250252. 
Butt, H.-J. (2007). Analyzing Electric Double Layers with the Atomic Force Microscope. In Encyclopedia of Electrochemistry, (Weinheim, Germany: Wiley-VCH Verlag GmbH \& Co. KGaA), pp. 225-248.

Butt, H.J., Cappella, B., and Kappl, M. (2005). Force measurements with the atomic force microscope: Technique, interpretation and applications. Surf. Sci. Rep. 59, 1-152.

Cao, Q., Zuo, C., Li, L., and Zhang, Y. (2012). Modulation of electroosmotic flow by electric fieldresponsive polyelectrolyte brushes: a molecular dynamics study. Microfluid Nanofluid 12, 649-655. Cerci, Y. (2002). Exergy analysis of a reverse osmosis desalination plant in California. Desalination 142, 257-266.

Chan Lee, S., Some, S., Wook Kim, S., Jun Kim, S., Seo, J., Lee, J., Lee, T., Ahn, J.-H., Choi, H.J., and Chan Jun, S. (2015). Efficient Direct Reduction of Graphene Oxide by Silicon Substrate. Sci. Rep. 5, 12306.

Chang, T.M.S. (1977). Biomedical Applications of Immobilized Enzymes and Proteins (Boston, MA: Springer US).

Chang, Y., Chang, W., Shih, Y., Wei, T., and Hsiue, G. (2011). Zwitterionic Sulfobetaine-Grafted Poly(vinylidene fluoride) Membrane with Highly Effective Blood Compatibility via Atmospheric PlasmaInduced Surface Copolymerization. Appl. Mater. Interfaces 3, 1228-1237.

Chavez-Valdez, A., Shaffer, M.S.P., and Boccaccini, A.R. (2013). Applications of graphene electrophoretic deposition. A review. J. Phys. Chem. B 117, 1502-1515.

Chávez-Valdez, A., and Boccaccini, A.R. (2012). Innovations in electrophoretic deposition: Alternating current and pulsed direct current methods. Electrochim. Acta 65, 70-89.

Chen, S., Zheng, J., Li, L., and Jiang, S. (2005). Strong Resistance of Phosphorylcholine SelfAssembled Monolayers to Protein Adsorption : Insights into Nonfouling Properties of Zwitterionic Materials. J. Am. Chem. Soc. 127, 14473-14478.

Chen, Y., Tao, J., Ezzeddine, A., Mahfouz, R., Al-Shahrani, A., Alabedi, G., and Khashab, N. (2015). Superior Performance Nanocomposites from Uniformly Dispersed Octadecylamine Functionalized Multi-Walled Carbon Nanotubes. C 1, 58-76.

Cheng, Y.L., and Lee, T.Y. (1992). Separation of fructose and glucose mixture by zeolite Y. Biotechnol. Bioeng. 40, 498-504.

Chou, Y., Venault, A., Cho, C., Sin, M., Yeh, L., Jhong, J., Chinnathambi, A., Chang, Y., and Chang, Y. (2017). Epoxylated Zwitterionic Triblock Copolymers Grafted onto Metallic Surfaces for General Biofouling Mitigation. Langmuir 33, 9822-9835.

Cohen, I., Avraham, E., Noked, M., Soffer, A., and Aurbach, D. (2011). Enhanced Charge Efficiency in Capacitive Deionization Achieved by Surface-Treated Electrodes and by Means of a Third Electrode. J. Phys. Chem. C 115, 19856-19863.

Cole, M.A., Voelcker, N.H., and Thissen, H. (2007). Electro-induced protein deposition on lowfouling surfaces. Smart Mater. Struct. 16, 2222-2228.

Collins, C.J., and Arrigan, D.W.M. (2009). A review of recent advances in electrochemically modulated extraction methods. Anal Bioanal Chem 393, 835-845.

Coskun, O. (2016). Separation Tecniques: Chromatography. North. Clin. Istanbul 3, 156-160. 
Cuatrecasas, P. (1970). Protein Purification by Affinity Chromatography. J. Biol. Chem. 245, 30593065.

Dahanayaka, M., Liu, B., Hu, Z., Chen, Z., Law, A.W., and Zhou, K. (2017). Corrugated graphene layers for sea water desalination using capacitive deionization. Phys. Chem. Chem. Phys 19, 85528562.

Deinhammer, R.S., Porter, M.D., and Shimazu, K. (1995). Retention characteristics of polypyrrole as a stationary phase for the electrochemically modulated liquid chromatographic (EMLC) separations of dansyl amino acids. J. Electroanal. Chem. 387, 35-46.

Deng, J., Xiong, T., Xu, F., Li, M., Han, C., Gong, Y., Wang, H., and Wang, Y. (2015). Inspired by bread leavening: one-pot synthesis of hierarchically porous carbon for supercapacitors. Green Chem. 17, 4053-4060.

Deore, B., Chen, Z., and Nagaoka, T. (2000). Potential-Induced Enantioselective Uptake of Amino Acid into Molecularly Imprinted Overoxidized Polypyrrole. Anal. Chem. 72, 3989-3994.

Diba, M., García-Gallastegui, A., Klupp Taylor, R.N., Pishbin, F., Ryan, M.P., Shaffer, M.S.P., and Boccaccini, A.R. (2014). Quantitative evaluation of electrophoretic deposition kinetics of graphene oxide. Carbon N. Y. 67, 656-661.

Diba, M., Fam, D.W.H., Boccaccini, A.R., and Shaffer, M.S.P. (2016). Electrophoretic deposition of graphene-related materials: A review of the fundamentals. Prog. Mater. Sci. 82, 83-117.

Dincer, I. (2002). The role of exergy in energy policy making. Energy Policy 30, 137-149.

Dincer, I., and Rosen, M.A. (2013). Exergy and Energy Analyses. In Exergy, (Elsevier), pp. 21-30.

Długołęcki, P., and Wal, A. Van Der (2013). Energy Recovery in Membrane Capacitive Deionization.

Environ. Sci. Technol. 47, 4904-4910.

Dreyer, D.R., Park, S., Bielawski, C.W., and Ruoff, R.S. (2010). The chemistry of graphene oxide. Chem. Soc. Rev. 39, 228-240.

Du, X., Zhang, H., Hao, X., Guan, G., and Abudula, A. (2014). Facile Preparation of Ion-Imprinted Composite Film for Selective Electrochemical Removal of Nickel (II) lons. Appl. Mater. Interfaces 6 , 9543-9549.

Duval, J., Lyklema, J., Kleijn, J.M., and van Leeuwen, H.P. (2001). Amphifunctionally Electrified Interfaces: Coupling of Electronic and lonic Surface-Charging Processes. Langmuir 17, 7573-7581. Dykstra, J.E., Zhao, R., Biesheuvel, P.M., and Wal, A. Van Der (2016). Resistance identification and rational process design in Capacitive Deionization. Water Res. 88, 358-370.

Eads, E.A., and Payne, D.A. (1964). Electrochemical Fractionation : Potentiostatic Chromatography and Elution Voltammetry X-Ray Fluorescence Spectrometric Analysis of Rubidium ( I ) and Cesium ( I ) Salts of 5-Nitrobarbituric Acid. Anal Chem 36, 2371-2372.

Ebeling, D., Ende, D. Van Den, and Mugele, F. (2011). Electrostatic interaction forces in aqueous salt solutions of variable concentration and valency. Nanotechnology 22, 1-10.

El-Deen, A.G., Boom, R.M., Kim, H.Y., Duan, H., Chan-Park, M.B., and Choi, J.H. (2016a). Flexible 3D Nanoporous Graphene for Desalination and Bio-decontamination of Brackish Water via Asymmetric Capacitive Deionization. ACS Appl. Mater. Interfaces 8, 25313-25325. 
El-Deen, A.G., Boom, R.M., Kim, H.Y., Duan, H., Chan-Park, M.B., and Choi, J.-H. (2016b). Flexible 3D Nanoporous Graphene for Desalination and Bio-decontamination of Brackish Water via Asymmetric Capacitive Deionization. ACS Appl. Mater. Interfaces 8, 25313-25325.

Elofsson, U.M., Paulsson, M.A., and Arnebrant, T. (1997). Adsorption of b-Lactoglobulin A and B in Relation to Self-Association: Effect of Concentration and pH. Langmuir 7463, 1695-1700.

Erdmann, M., David, R., Fornof, A., and Gaub, H.E. (2009). Electrically controlled DNA adhesion. Nat. Nanotechnol. 5, 154-159.

Espenscheid, M.W., and Martin, C.R. (1985). Electroactive ion exchange polymers. J. Electroanal. Chem. 188, 73-84.

Espenscheid, M.W., and Martin, C.R. (1989). Ion Exchange Voltammetry with Electroactive lonomers. Electroanalysis 1, 93-95.

Ezzat, M., and Huang, C. (2016). Zwitterionic polymer brush coatings with excellent anti-fog and antifrost properties. RSC Adv. 6, 61695-61702.

Fainerman, V.B., Miller, R., Ferri, J.K., Watzke, H., Leser, M.E., and Michel, M. (2006). Reversibility and irreversibility of adsorption of surfactants and proteins at liquid interfaces. Adv. Colloid Interface Sci. 123-126, 163-171.

Fang, F., and Szleifer, I. (2006). Controlled release of proteins from polymer-modified surfaces. PNAS 103, 5769-5774.

Farmer, J.C., Fix, D. V., Mack, G., Pekala, R.W., and Poco, J.F. (1996a). Capacitive Deionization of $\mathrm{NaCl}$ and NaNO3 Solutions with Carbon Aerogel Electrodes. J. Electrochem. Soc. 143, 159.

Farmer, J.C., Fix, D. V, Mack, G. V, Pekala, R.W., and Poco, J.F. (1996b). Capacitive deionization of $\mathrm{NH} 4 \mathrm{ClO} 4$ solutions with carbon aerogel electrodes. J. Appl. Electrochem. 26, 1007-1018.

Fermín, D.J. (2006). Nanoscale Probing of Electrode Surfaces by Scanning Force Microscopy. 60, 789-794.

Ferrari, B., and Moreno, R. (1997). Electrophoretic deposition of aqueous alumina slips. J. Eur.

Ceram. Soc. 17, 549-556.

Fitzsimons, L. (2011). A Detailed Study of Desalination Exergy Models and their Application to a Semiconductor Ultra-Pure Water Plant. Dublin City University.

Fitzsimons, L., Corcoran, B., Young, P., Foley, G., and Regan, F. (2012). Modelling the Activity of Seawater and Implications for Desalination Exergy Analyses. In 9th International Conference on Heat Transfer, Fluid Mechanics and Thermodynamics, (Malta), p.

Fitzsimons, L., Corcoran, B., Young, P., and Foley, G. (2015). Exergy analysis of water purification and desalination: A study of exergy model approaches. Desalination 359, 212-224.

Fomina, N., Johnson, C.A., Maruniak, A., Bahrampour, S., Lang, C., Davis, R.W., Kavusi, S., and Ahmad, H. (2016). An electrochemical platform for localized pH control on demand. Lab Chip 16, 2236-2244.

Frasconi, M., Tel-Vered, R., Elbaz, J., and Willner, I. (2010). Electrochemically stimulated pH changes: A route to control chemical reactivity. J. Am. Chem. Soc. 132, 2029-2036.

Fréchette, J., and Vanderlick, T.K. (2001). Double Layer Forces over Large Potential Ranges as Measured in an Electrochemical Surface Forces Apparatus. Langmuir 17, 7620-7627. 
Fritz, P.A., Zisopoulos, F.K., Verheggen, S., Schroën, K., and Boom, R.M. (2018). Exergy analysis of membrane capacitive deionization (MCDI). Desalination 444, 162-168.

Fritz, P.A., Boom, R.M., and Schroen, K. (2019a). Polyelectrolyte-activated carbon composite electrodes for inverted membrane capacitive deionization (iMCDI). Sep. Purif. Technol. 220, 145-151. Fritz, P.A., Lange, S.C., Giesbers, M., Zuilhof, H., Boom, R.M., and Schroen, C.G.P.H. (2019b). Simultaneous Silicon Oxide Growth and Electrophoretic Deposition of Graphene Oxide. Langmuir 35, 3717-3723.

Fritzmann, C., Löwenberg, J., Wintgens, T., and Melin, T. (2007). State-of-the-art of reverse osmosis desalination. Desalination 216, 1-76.

Fu, Y., Zhu, J., Hu, C., Wu, X., and Wang, X. (2014). Covalently coupled hybrid of graphitic carbon nitride with reduced graphene oxide as a superior performance lithium-ion battery anode. Nanoscale 6, 12555-12564.

Fuciños, C., Fuciños, P., Estévez, N., Pastrana, L.M., Vicente, A.A., and Luisa, M. (2019). Onestep chromatographic method to purify a -lactalbumin from whey for nanotube synthesis purposes. Food Chem. 275, 480-488.

Gaikwad, M.S., and Balomajumder, C. (2016). Polymer coated Capacitive Deionization Electrode for Desalination: A mini review. Electrochem. Energy Technol. 2, 1-5.

Galanakis, C.M. (2012). Recovery of high components from food wastes : Conventional , emerging technologies and commercialized applications. Trends Food Sci. Technol. 26, 68-87.

Gao, W. (2015). Graphene Oxide - Reduction recipes, spectroscopy, and applications (Cham: Springer International Publishing).

Gao, K., Shao, Z., Wu, X., Wang, X., Li, J., Zhang, Y., Wang, W., and Wang, F. (2013). Cellulose nanofibers/reduced graphene oxide flexible transparent conductive paper. Carbohydr. Polym. 97, 243-251.

Gao, Q., Li, P., Zhao, H., Chen, Y., Jiang, L., and Ma, P.X. (2017a). Methacrylate-ended polypeptides and polypeptoids for antimicrobial and antifouling coatings. Polym. Chem. 8, 6386-6397. Gao, X., Omosebi, A., Landon, J., and Liu, K. (2015a). Enhanced Salt Removal in an Inverted Capacitive Deionization Cell Using Amine Modified Microporous Carbon Cathodes. Environ. Sci. Technol. 49, 10920-10926.

Gao, X., Omosebi, A., Landon, J., and Liu, K. (2015b). Surface charge enhanced carbon electrodes for stable and efficient capacitive deionization using inverted adsorption-desorption behvior. Energy Environ. Sci. 8, 897-909.

Gao, X., Porada, S., Omosebi, A., Liu, K., Biesheuvel, P.M., and Landon, J. (2016a). Complementary surface charge for enhanced capacitive deionization. Water Res. 92, 275-282.

Gao, X., Omosebi, A., Holubowitch, N., Liu, A., Ruh, K., Landon, J., and Liu, K. (2016b). Polymercoated composite anodes for efficient and stable capacitive deionization. Desalination 399, 16-20.

Gao, X., Omosebi, A., Holubowitch, N., Landon, J., and Liu, K. (2017b). Capacitive Deionization Using Alternating Polarization : Effect of Surface Charge on Salt Removal. Electrochim. Acta 233, 249-255. 
Gao, X., Omosebi, A., Landon, J., and Liu, K. (2018). Voltage-Based Stabilization of Microporous Carbon Electrodes for Inverted Capacitive Deionization. J. Phys. Chem. C 122, 1158-1168.

García-Quismondo, E., Santos, C., Lado, J., Palma, J., and Anderson, M.A. (2013). Optimizing the Energy Efficiency of Capacitive Deionization Reactors Working under Real-World Conditions. Environ. Sci. Technol. 47, 11866-11872.

García, U.P., Ibanez, J.G., and Batina, N. (2011). Electrochemical Modulation of the Thickness of Polypyrrole Films by Using Different Anionic Dopants. Internatioal J. Electrochem. Sci. 6, 5172-5188.

Ge, H., and Wallace, G. (1992). Ion exchange properties of polypyrrole. React. Polym. 18, 133-140.

Ge, H., Teasdale, P., and Wallace, G. (1991). Electrochemical chromatography -packings , mechanisms of interaction. J. Chromatogr. 544, 305-316.

Geissler, C., and Powers, H.J. (2012). Human Nutrition (Elsevir).

Genborg, A., and Sharp, M. (1993). Studies of mediated oxidation reactions of $\mathrm{Fe}(\mathrm{CN}) 4-6$,

$\mathrm{Ru}(\mathrm{CN}) 4-6$ and catechol at glassy-carbon electrodes coated with thin films of poly-

(styrenesulphonate) containing Os(bipy)3+/2+3 redox sites. Electrochim. Acta 38, 1321-1329.

Geng, X., and Wang, L. (2008). Liquid chromatography of recombinant proteins and protein drugs. J. Chromatogr. B Anal. Technol. Biomed. Life Sci. 866, 133-153.

Gerberding, S.J., and Byers, C.H. (1998). Preparative ion-exchange chromatography of proteins from dairy whey. J. Chromatogr. A 808, 141-151.

Gibbs, J.W. (1878). On the equilibrium of heterogeneous substances. Am. J. Sci. s3-16, 441-458.

Giddings, J.C. (1991). Unified separation science (New York: Wiley).

Giesbers, M., Kleijn, J.M., and Cohen Stuart, M.A. (2002). The electrical double layer on gold probed by electrokinetic and surface force measurements. J. Colloid Interface Sci. 248, 88-95. van der Goot, A.J., Pelgrom, P.J.M., Berghout, J.A.M., Geerts, M.E.J., Jankowiak, L., Hardt, N.A., Keijer, J., Schutyser, M.A.I., Nikiforidis, C. V, and Boom, R.M. (2016). Concepts for further sustainable production of foods. J. Food Eng. 168, 42-51.

Gräslund, S., Nordlund, P., Weigelt, J., Bray, J., Gileadi, O., Knapp, S., Oppermann, U., Arrowsmith, C., Hui, R., Ming, J., et al. (2008). Protein production and purification. Nat. Methods 5 , 135-146.

Grumelli, D.E., Garay, F., Barbero, C.A., and Calvo, E.J. (2006). Dynamics of Ion Exchange between Self-assembled Redox Polyelectrolyte Multilayer Modified Electrode and Liquid Electrolyte. J. Phys. Chem. B 110, 15345-15352.

Guo, Z., and Zheng, S. (2009). Separation of p-chloronitrobenzene and o-chloronitrobenzene by selective adsorption using Silicalite-1 zeolite. Chem. Eng. Jour 155, 654-659.

Hage, D.S., Anguizola, J.A., Bi, C., Li, R., Matsuda, R., Pfaunmiller, E., Vargas, J., and Zheng, X. (2012). Pharmaceutical and biometical applications of affinity chromatography: Recent trends and developments. J Pharm Biomed Anal 69, 93-105.

Han, L., Karthikeyan, K.G., and Gregory, K.B. (2015). Energy Consumption and Recovery in Capacitive Deionization Using Nanoporous Activated Carbon Electrodes. J. Electrochem. Soc. 162, E282-E288. 
Harnisch, J.A., and Porter, M.D. (2001). Electrochemically modulated liquid chromatography: an electrochemical strategy for manipulating chromatographic retention. Analyst 126, 1841-1849.

Hasan, S.A., Rigueur, J.L., Harl, R.R., Krejci, A.J., Gonzalo-Juan, I., Rogers, B.R., and Dickerson, J.H. (2010). Transferable Graphene Oxide Films with Tunable Microstructures. ACS Nano 4, 7367-7372.

Hasan, T., Gazda, D., Porter, M.D., and Houk, R.S. (2005). On-line cleanup of blanks by electrochemically modulated liquid chromatography for inductively coupled plasma-mass spectrometry. Anal. Chim. Acta 537, 63-68.

Hatton, F.L., Engström, J., Forsling, J., Malmström, E., and Carlmark, A. (2017). Biomimetic adsorption of zwitterionic-xyloglucan block copolymers to CNF: towards tailored super-absorbing cellulose materials. RSC Adv. 7, 14947-14958.

He, Y., Hower, J., Chen, S., Bernards, M.T., Chang, Y., and Jiang, S. (2008). Molecular Simulation Studies of Protein Interactions with Zwitterionic Phosphorylcholine Self-Assembled Monolayers in the Presence of Water. Langmuir 24, 10358-10364.

Hemmatifar, A., Palko, J.W., Stadermann, M., and Santiago, J.G. (2016). Energy breakdown in capacitive deionization. Water Res. 104, 303-311.

Henrie, J., Kellis, S., Schultz, S., and Hawkins, A. (2004). Electronic color charts for dielectric films on silicon. Opt. Express 12, 1464-1469.

Hepel, M., and Dentrone, L. (1996). Controlled Incorporation of Heavy Metals from Aqueous Solutions and Their Electrorelease Using Composite Polypyrrole Films. Electroanalysis 8, 996.

Herden, H., Einicke, W.-D., Jusek, M., Messow, U., and Schöller, R. (1984). Adsorption Studies of n-Olefin/n-Paraffin Mixtures on X- and Y-Zeolites. J. Colloid Interface Sci. 97, 559-564.

Hevert, H.W., and Hevert, S.C. (1980). Second law analysis: An alternative indicator of system efficiency. Energy 5, 865-873.

Higgins, S.J. (1997). Conjugated polymers incorporating pendant functional groups-synthesis and characterisation. Chem. Soc. Rev. 26, 247-257.

Hilbrig, F., and Freitag, R. (2003). Protein purification by affinity precipitation. J. Chromatogr. B 790, 79-90.

Hillier, A.C., Kim, S., and Bard, A.J. (1996). Measurement of Double-Layer Forces at the Electrode/Electrolyte Interface Using the Atomic Force Microscope: Potential and Anion Dependent Interactions. J. Phys. Chem. 3654, 18808-18817.

Hillman, A.R., Hughes, N.A., and Bruckenstein, S. (1992). Solvation Phenomena in Polyvinylferrocene Films: Effect of History and Redox State. J. Electrochem. Soc. 139, 74-77. Hodneland, C.D., and Mrksich, M. (2000). Biomolecular Surfaces that Release Ligands under Electrochemical Control. J. Am. Chem. Soc. 122, 4235-4236.

Hokkanen, S., and Bhatnagar, A. (2016). A review on modification methods to cellulose-based adsorbents to improve adsorption capacity. Water Res. 91, 156-173.

Holmstrom, N., Askendal, A., and Tengvall, P. (1998). In vitro studies on blood protein adsorption to gold and pyrolytic carbon at pre-set electrical potentials. Colloids Surfaces B Biointerfaces $11,265-$ 271. 
Hook, A.L., Thissen, H., Hayes, J.P., and Voelcker, N.H. (2006). Spatially controlled electrostimulated DNA adsorption and desorption for biochip applications. 21, 2137-2145.

Hu, K., Fan, F.F., Bard, A.J., and Hillier, A.C. (1997). Direct Measurement of Diffuse Double-Layer Forces at the Semiconductor / Electrolyte Interface Using an Atomic Force Microscope. 5647, 82988303.

Huang, W.E.I., Zhang, Y., and Bao, S. (2013). Desalination by capacitive deionization with carbonbased materials as electrode: A review. Surf. Rev. Lett. 20, 1-10.

Huang, X., Zeng, Z., Fan, Z., Liu, J., and Zhang, H. (2012). Graphene-based electrodes. Adv. Mater. 24, 5979-6004.

Huang, Z.-H., Yang, Z., Kang, F., and Inagaki, M. (2017). Carbon electrodes for capacitive deionization. J. Mater. Chem. A 5, 470-496.

Hui, S., and Yang, Y. (2017). Dual-ions electrochemical deionization: a desalination generator.

Energy Environ. Sci. 10, 2081-2089.

Huyskens, C., Helsen, J., Groot, W.J., and De Haan, A.B. (2013). Membrane capacitive deionization for biomass hydrolysate desalination. Sep. Purif. Technol. 118, 33-39.

Inglezakis, V.J., and Zorpas, A.A. (2012). Heat of adsorption, adsorption energy and activation energy in adsorption and ion exchange systems. Desalin. Water Treat. 39, 149-157.

Ishikawa, R., Ko, P.J., Kurokawa, Y., Konagai, M., and Sandhu, a (2012). Electrophoretic deposition of high quality transparent conductive graphene films on insulating glass substrates. J. Phys. Conf. Ser. 352, 012003.

Israelachvili, J.N. (2011). Intermolecular and Surface Forces (Elsevier).

Israelachvili, J.N., and Pashley, R.M. (1983). Molecular layering of water at surfaces and origin of repulsive hydration forces. Nature 306, 249-250.

Jayathilakan, K., Sultana, K., Radhakrishna, K., and Bawa, A.S. (2012). Utilization of byproducts and waste materials from meat, poultry and fish processing industries : a review. J Food Sci Technol 49, 278-293.

Jenkins, G.H. (1966). Introduction to cane sugar technology (Elsevier).

Ji, Y., An, Q., Zhao, Q., Sun, W., Lee, K., Chen, H., and Gao, C. (2012). Novel composite nanofiltration membranes containing zwitterions with high permeate flux and improved anti-fouling performance. J. Memb. Sci. 390-391, 243-253.

Jia, B., and Zhang, W. (2016). Preparation and Application of Electrodes in Capacitive Deionization (CDI): a State-of-Art Review. Nanoscale Res. Lett. 11, 64.

Jiang, M., Ray, W.W., Mukherjee, B., and Wang, J. (2004). Electrochemically controlled release of lipid/DNA complexes: a new tool for synthetic gene delivery system. Electrochem. Commun. 6, 576582.

Jung, S., Choi, J., and Kim, J. (2012). Application of capacitive deionization (CDI) technology to insulin purification process. Sep. Purif. Technol. 98, 31-35.

Jureviciute, I., Bruckenstein, S., and Hillman, A.R. (2000). Counter-ion specific effects on charge and solvent trapping in poly(vinylferrocene) films. J. Electroanal. Chem. 488, 73-81. 
Kahraman, N., Cengel, Y.A., Wood, B., and Cerci, Y. (2004). Exergy analysis of a combined RO, NF, and EDR desalination plant Nafiz. Desalination 171, 217-232.

Kang, J., Kim, T., Shin, H., Lee, J., Ha, J., and Yoon, J. (2016). Direct energy recovery system for membrane capacitive deionization. Desalination 398, 144-150.

Kanoh, H., Ooi, K., Miyai, Y., and Katoh, S. (1991). Selective Electroinsertion of Lithium lons into a $\mathrm{Pt} / \mathrm{I}-\mathrm{MnO} 2$ Electrode in Aqueous Phase. Langmuir 7, 1841-1842.

Kao, L.T., Hsu, H., and Gratzl, M. (2008). Reagentless pH-stat for Microliter Fluid Specimens. Anal. Chem. 80, 4065-4069.

Karthick, R., Brindha, M., Selvaraj, M., and Ramu, S. (2013). Stable colloidal dispersion of functionalized reduced graphene oxide in aqueous medium for transparent conductive film. J. Colloid Interface Sci. 406, 69-74.

Kawaguchi, M., Hayashi, K., and Takahashi, A. (1988). Effect of applied potential on the ellipsometric thickness of sodium poly(styrenesulfonates) adsorbed on a platinum surface. Macromolecules 21, 1016-1020.

Khan, G.F., and Wernet, W. (1997). Adsorption of proteins on electro-conductive polymer films. Thin Solid Films 300, 265-271.

Kilpatrick, J.I., Loh, S., and Jarvis, S.P. (2013). Directly Probing the Effects of lons on Hydration Forces at Interfaces. J. Am. Chem. Soc. 135, 2628-2634.

Kim, Y.J., and Choi, J.H. (2012). Selective removal of nitrate ion using a novel composite carbon electrode in capacitive deionization. Water Res. 46, 6033-6039.

Kim, S., Choi, J., and Kim, J. (2012). Removal of acetic acid and sulfuric acid from biomass hydrolyzate using a lime addition - capacitive deionization (CDI) hybrid process. Process Biochem. 47, 2051-2057.

Kim, S., Lee, J., Kim, C., and Yoon, J. (2016). Na2FeP2O7 as a Novel Material for Hybrid Capacitive Deionization. Electrochim. Acta 203, 265-271.

Kim, S., Yoon, H., Shin, D., Lee, J., and Yoon, J. (2017). Electrochemical selective ion separation in capacitive deionization with sodium manganese oxide. J. Colloid Interface Sci. 506, 644-648.

Kim, Y.J., Kim, J.H., and Choi, J.H. (2013). Selective removal of nitrate ions by controlling the applied current in membrane capacitive deionization (MCDI). J. Memb. Sci. 429, 52-57.

Kleijn, J.M., Barten, D., and Cohen Stuart, M.A. (2004). Adsorption of charged macromolecules at a gold electrode. Langmuir 20, 9703-9713.

Kokh, D.B., Corni, S., Winn, P.J., Hoefling, M., Gottschalk, K.E., and Wade, R.C. (2010).

ProMetCS : An Atomistic Force Field for Modeling Protein - Metal Surface Interactions in a Continuum Aqueous Solvent. 1753-1768.

Kou, L., and Gao, C. (2011). Making silica nanoparticle-covered graphene oxide nanohybrids as general building blocks for large-area superhydrophilic coatings. Nanoscale 3, 519-528.

Kubáň, P., Šlampová, A., and Boček, P. (2010). Electric field-enhanced transport across phase boundaries and membranes and its potential use in sample pretreatment for bioanalysis.

Electrophoresis 31, 768-785.

Kumar, S., Kumar, S., Srivastava, S., Yadav, B.K., Lee, S.H., Sharma, J.G., Doval, D.C., and 
Malhotra, B.D. (2015). Reduced graphene oxide modified smart conducting paper for cancer biosensor. Biosens. Bioelectron. 73, 114-122.

Kuznetsov, V., and Papastavrou, G. (2012). Adhesion of Colloidal Particles on Modi fi ed Electrodes. Langmuir 28, 16567-16579.

Lahann, J., Mitragotri, S., Tran, T.-N., Kaido, H., Sundaram, J., Choi, I.S., Hoffer, S., Somorjai, G.A., and Langer, R. (2003). A Reversibly Switching Surface. Science (80-. ). 299, 371-374.

Lake, J.R., Cheng, A., and Selverston, S. (2012). Graphene metal oxide composite supercapacitor electrodes. J. Vac. Sci. Technol. B 30, 03D118.

Lam, P., Elliker, P.R., Wnek, G.E., and Przybycien, T.M. (1995). Towards an electrochemically modulated chromatographic stationary phase. J. Chromatogr. A 707, 29-33.

Lange, S.C., van Andel, E., Smulders, M.M.J., and Zuilhof, H. (2016). Efficient and Tunable ThreeDimensional Functionalization of Fully Zwitterionic Antifouling Surface Coatings. Langmuir 32, 1019910205.

Lee, J., Park, K., Eum, H., and Lee, C. (2006). Desalination of a thermal power plant wastewater by membrane capacitive deionization. Desalination 196, 125-134.

Lee, J., Kim, S., and Yoon, J. (2017). Rocking Chair Desalination Battery Based on Prussian Blue Electrodes. ACS Omega 2, 1653-1659.

Li, H., Pan, L., Lu, T., Zhan, Y., Nie, C., and Sun, Z. (2011). A comparative study on electrosorptive behavior of carbon nanotubes and graphene for capacitive deionization. J. Electroanal. Chem. 653, 40-44.

Li, Z., Song, B., Wu, Z., Lin, Z., Yao, Y., Moon, K., and Wong, C.P. (2015). 3D porous graphene with ultrahigh surface area for microscale capacitive deionization. Nano Energy 11, 711-718.

Liang, H., Bu, Y., Zhang, J., Cao, Z., and Liang, A. (2013). Graphene oxide film as solid lubricant. ACS Appl. Mater. Interfaces 5, 6369-6375.

Liang, Q., Ye, L., Huang, Z., Xu, Q., Bai, Y., Kang, F., and Yang, Q. (2014). A honeycomb-like porous carbon derived from pomelo peel for use in high-performance supercapacitors. Nanoscale 6 , 13831-13837.

Liljegren, G., and Nyholm, L. (2003). Electrochemically controlled solid-phase microextraction and preconcentration using polypyrrole coated microarray electrodes in a flow system. Analyst $128,232-$ 236.

Liljegren, G., Pettersson, J., Markides, K.E., and Nyholm, L. (2002). Electrochemical solid-phase microextraction of anions and cations using polypyrrole coatings and an integrated three-electrode device. Analyst 127, 591-597.

Lindall, C.M., Crayston, J.A., Cole-hamilton, D.J., Glidleb, A., and Peacockb, R.D. (1996).

Preparation, voltammetry, coulometry and electrochemical quartz crystal gravimetry of [Os(bipy)2(PVPS)nCIRu(edta)] redox polymers. J. Mater. Chem. 6, 1259-1269.

Liu, L., Guo, X., Tallon, R., Huang, X., and Chen, J. (2017). Highly porous N-doped graphene nanosheets for rapid removal of heavy metals from water by capacitive deionization. ChemComm 53 , 881-884. 
Liu, X., Wang, X., Tan, F., Zhao, H., Quan, X., Chen, J., and Li, L. (2012). An electrochemically enhanced solid-phase microextraction approach based on molecularly imprinted polypyrrole / multiwalled carbon nanotubes composite coating for selective extraction of fluoroquinolones in aqueous samples. Anal. Chim. Acta 727, 26-33.

Liu, Y., Mu, L., Liu, B., Zhang, S., Yang, P., and Kong, J. (2004). Controlled protein assembly on a switchable surfaceElectronic supplementary information (ESI) available: preparation and characterization of LD-MHA-SAM-n and the assembled protein films. See http://www.rsc.org/suppdata/cc/b4/b400776j/. Chem. Commun. 0, 1194.

Liu, Y., Chang, Y., Chang, Y., and Shih, Y. (2010). Preparation of Amphiphilic PolymerFunctionalized Carbon Nanotubes for Low-Protein-Adsorption Surfaces and Protein-Resistant Membranes. Appl. Mater. Interfaces 2, 3642-3647.

Liu, Y., Meng, X., Liu, Z., Meng, M., Jiang, F., Luo, M., Ni, L., Qiu, J., Liu, F., and Zhong, G. (2015). Preparation of a Two-Dimensional lon-Imprinted Polymer Based on a Graphene Oxide/SiO2 Composite for the Selective Adsorption of Nickel lons. Langmuir 31, 8841-8851.

Lu, D.R., Lee, S.J., and Park, K. (1992). Calculation of solvation interaction energies for protein adsorption on polymer surfaces. J. Biomater. Sci. Polym. Ed. 3, 127-147.

Lyklema, J. (2000). Fundamentals of Interface and Colloid Science (Academic Press).

Ma, X., and Shrotriya, P. (2012). Molecular Dynamics simulation of electrical field induced conformational transition and associated frictional performance of monomolecular films. J. Phys. D. Appl. Phys. 45, 375306.

Maharjan, P., Woonton, B.W., Bennett, L.E., Smithers, G.W., DeSilva, K., and Hearn, M.T.W. (2008). Novel chromatographic separation - The potential of smart polymers. Innov. Food Sci. Emerg. Technol. 9, 232-242.

Mailliart, P., and Ribadeau-Dumas, B. (1988). Preparation of b-Lactoglobulin and b-LactoglobulinFree Proteins from Whey Retentate by $\mathrm{NaCl}$ Salting Out at Low pH. J. Food Sci. 53, 743-745.

Mandal, S., and Kulkarni, B.D. (2011). Separation Strategies for Processing of Dilute Liquid Streams. Int. J. Chem. Eng. 2011, 1-19.

Marsh, R.J., Jones, R.A.L., and Sferrazza, M. (2002). Adsorption and displacement of a globular protein on hydrophilic and hydrophobic surfaces. Colloids Surfaces B Biointerfaces 23, 31-42.

Mattson, J.S., and Smith, C.A. (1973). Enhanced Protein Adsorption at the Solid-Solution Interface: Dependence on Surface Charge. Science (80-. ). 181, 1055-1057.

Mcumber, A.C., Randolph, T.W., and Schwartz, D.K. (2015). Electrostatic Interactions Influence Protein Adsorption (but Not Desorption) at the Silica - Aqueous Interface. J. Phys. Chem. Lett. 6, 2583-2587.

Mechanics, F., Fitzsimons, L., and Sensing, E. (2012). Modelling the Activity of Seawater and Implications for Desalination Exergy Analyses.

Mekonnen, M.M., and Hoekstra, A.Y. (2016). Four billion people facing severe water scarcity. Sci. Adv. 2, e1500323.

Mendes, P.M. (2008). Stimuli-responsive surfaces for bio-applications. Chem. Soc. Rev. 37, 25122529. 
Mi, Y., Zhao, Q., Ji, Y., An, Q., and Gao, C. (2015). A novel route for surface zwitterionic functionalization of polyamide nano fi Itration membranes with improved performance. J. Memb. Sci. 490, 311-320.

Miller, L.L., and Zhou, X.Q. (1987). Poly(N-methylpyrrolylium) poly(styrenesulfonate) - a conductive, electrically switchable cation exchanger that cathodically binds and anodically releases dopamine. Macromolecules 20, 1594-1597.

Morales-Cid, G., Cárdenas, S., Simonet, B.M., and Valcárcel, M. (2010). Sample treatments improved by electric fields. TrAC Trends Anal. Chem. 29, 158-165.

Morimoto, K., Toya, M., Fukuda, J., and Suzuki, H. (2008). Automatic Electrochemical Micro-pHStat for. Anal. Chem. 80, 905-914.

Morr, C. V, and Ha, E.Y.W. (1993). Whey Protein Concentrates and Isolates: Processing and Functional Properties. Crit. Rev. Food Sci. Nutr. 33, 431-476.

Morrissey, B.., Smith, L.E., Stromberg, R.., and Fenstermaker, C.A. (1976). Ellipsometric investigation of the effect of potential on blood protein conformation and adsorbance. J. Colloid Interface Sci. 56, 557-563.

Mossad, M., and Zou, L. (2013). Study of fouling and scaling in capacitive deionisation by using dissolved organic and inorganic salts. J. Hazard. Mater. 244-245, 387-393.

Moulton, S.E., Barisci, J.N., Bath, A., Stella, R., and Wallace, G.G. (2003). Investigation of protein adsorption and electrochemical behavior at a gold electrode. J. Colloid Interface Sci. 261, 312-319.

Mu, L., Liu, Y., Cai, S., and Kong, J. (2007). A smart surface in a microfluidic chip for controlled protein separation. Chem. - A Eur. J. 13, 5113-5120.

Mugele, F., and Baret, J. (2005). Electrowetting: from basics to applications. J. Phys. Condens. Matter 17, R705-R774.

Muskal, N., and Mandler, D. (2000). The electrochemistry of thiol self-assembled monolayers (SAMs) on a hanging mercury drop electrode (HMDE). Curr. Sep. 19, 49-54.

Nakanaishi, K., Sakiyama, T., and Imamura, K. (2001). On the Adsorption of Proteins on Solid Surfaces, a Common but Very Complicated Phenomenon. J. Biosci. Bioeng. 91, 233-244.

Nam, D., and Choi, K. (2017). Bismuth as a New Chloride-Storage Electrode Enabling the Construction of a Practical High Capacity Desalination Battery. J. Am. Chem. Soc. 139, 11055-11063.

Narayanan, S.R. (1994). Review Preparative affinity chromatography of proteins. J. Chromatogr. A 658, 237-258.

Neyestani, T.R., Djalali, M., and Pezeshki, M. (2003). Isolation of a-lactalbumin, b-lactoglobulin, and bovine serum albumin from cow's milk using gel filtration and anion-exchange chromatography including evaluation of their antigenicity. Protein Expr. Purif. 29, 202-208.

Ng, C.C.A., Magenau, A., Ngalim, S.H., Ciampi, S., Chockalingham, M., Harper, J.B., Gaus, K., and Gooding, J.J. (2012). Using an Electrical Potential to Reversibly Switch Surfaces between Two States for Dynamically Controlling Cell Adhesion. Angew. Commun. 51, 7706-7710.

Nikitas, P. (2000). Theory of electrochemically modulated liquid chromatography. 484, 137-143.

Norde, W. (1995). Adsorption of Proteins at Solid-Liquid Interfaces. Cells Mater. 5, 97-112. 
Norde, W., Arai, T., and Shirahama, H. (1991). Protein adsorption in model systems. Biofouling 4, 37-51.

Oladunni, J., Zain, J.H., Hai, A., Banat, F., Bharath, G., and Alhseinat, E. (2018). A comprehensive review on recently developed carbon based nanocomposites for capacitive deionization: From theory to practice. Sep. Purif. Technol. 207, 291-320.

Omanovic, S., and Roscoe, S.G. (2000). Interfacial Behavior of $\beta$-Lactoglobulin at a Stainless Steel Surface: An Electrochemical Impedance Spectroscopy Study. J. Colloid Interface Sci. 227, 452-460. Oren, Y. (2008). Capacitive deionization (CDI) for desalination and water treatment - past, present and future (a review). Desalination 228, 10-29.

Oschatz, M., Lee, J.T., Kim, H., Nickel, W., Borchardt, L., Cho, W.I., Ziegler, C., Kaskel, S., and Yushin, G. (2014). Micro- and mesoporous carbide-derived carbon prepared by a sacrificial template method in high performance lithium sulfur battery cathodes. J. Mater. Chem. A 2, 17649-17654.

Ouyang, H., Xia, Z., and Zhe, J. (2009). Static and dynamic responses of polyelectrolyte brushes under external electric field. Nanotechnology 20.

Oyarzun, D.I., Hemmatifar, A., Palko, J.W., Stadermann, M., and Santiago, J.G. (2018). Adsorption and capacitive regeneration of nitrate using inverted capacitive deionization with surfactant functionalized carbon electrodes. Sep. Purif. Technol. 194, 410-415.

Palko, J.W., Oyarzun, D.I., Ha, B., Stadermann, M., and Santiago, J.G. (2018). Nitrate removal from water using electrostatic regeneration of functionalized adsorbent. Chem. Eng. J. 334, 12891296.

Pandolfo, A.G., and Hollenkamp, A.F. (2006). Carbon properties and their role in supercapacitors. J. Power Sources 157, 11-27.

Papastavrou, G. (2010). Combining electrochemistry and direct force measurements: from the control of surface properties towards applications. Colloid Polym. Sci. 288, 1201-1214.

Park, J.H., and Park, J.M. (2014). Electrophoretic deposition of graphene oxide on mild carbon steel for anti-corrosion application. Surf. Coatings Technol. 254, 167-174.

Park, S., An, J., Potts, J.R., Velamakanni, A., Murali, S., and Ruoff, R.S. (2011). Hydrazinereduction of graphite- and graphene oxide. Carbon N. Y. 49, 3019-3023.

Parsegian, V.A., and Zemb, T. (2011). Hydration forces: Observations, explanations, expectations, questions. Curr. Opin. Colloid Interface Sci. 16, 618-624.

Parsons, D.F., Bostrom, M., Lo Nostro, P., and Ninham, B.W. (2011). Hofmeister effects: interplay of hydration, nonelectrostatic potentials, and ion size. Phys. Chem. Chem. Phys 13, 12352-12367.

Pascal, V., Laetitia, D., Joel, L., Marc, S., and Serge, P. (2007). New concept to remove heavy metals from liquid waste based on electrochemical $\mathrm{pH}$-switchable immobilized ligands. Appl. Surf. Sci. 253, 3263-3269.

Pasta, M., Wessells, C.D., Cui, Y., and Mantia, F. La (2012a). A Desalination Battery. Nano Lett. 12, 23-27.

Pasta, M., Battistel, A., and Mantia, F. La (2012b). Batteries for lithium recovery from brines. Energy Environ. Sci. 5, 9487-9491. 
Pattnaik, P. (2005). Surface Plasmon Resonance. Appl. Biochem. Biotechnol. 126, 79-92.

Pavitt, A.S., Bylaskab, E.J., and Tratnyek, P.G. (2017). Oxidation potentials of phenols and anilines: correlation analysis of electrochemical and theoretical values. Environ. Sci. Process Impacts 19, 339.

Pedersen-bjergaard, S., and Rasmussen, K.E. (2008). Electrical potential can drive liquid-liquid extraction for sample preparation in chromatography. Trends Anal. Chem. 27, 934-941.

Pedersen, L., Mollerup, J., Hansen, E., and Jungbauer, A. (2003). Whey proteins as a model system for chromatographic separation of proteins. J. Chromatogr. B 790, 161-173.

Pei, L., and Lucy, C.A. (2014). Insight into the stability of poly (diallydimethylammoniumchloride ) and polybrene poly cationic coatings in capillary electrophoresis. J. Chromatogr. A 1365, 226-233.

Petersen, N.J., Rasmussen, K.E., Pedersen-Bjergaard, S., and Gjelstad, A. (2011).

Electromembrane Extraction from Biological Fluids EME of Different Basic Drugs. Anal. Sci. 27, 965972.

Pham, V.H., Cuong, T.V., Hur, S.H., Shin, E.W., Kim, J.S., Chung, J.S., and Kim, E.J. (2010). Fast and simple fabrication of a large transparent chemically-converted graphene film by spray-coating. Carbon N. Y. 48, 1945-1951.

Porada, S., Zhao, R., Van Der Wal, A., Presser, V., and Biesheuvel, P.M. (2013). Review on the science and technology of water desalination by capacitive deionization. Prog. Mater. Sci. $58,1388-$ 1442.

Porter, M.D., and Takano, H.D. (2000). CHROMATOGRAPHY: LIQUID | Electrochemically Modulated Liquid Chromatography. In Encyclopedia of Separation Science, (Elsevier), pp. 636-646. Pranzetti, A., Mieszkin, S., Iqbal, P., Rawson, F.J., Callow, M.E., Callow, J.A., Koelsch, P., Preece, J.A., and Mendes, P.M. (2013). An electrically reversible switchable surface to control and study early bacterial adhesion dynamics in real-time. Adv. Mater. 25, 2181-2185.

Puthusseri, D., Aravindan, V., Madhavi, S., and Ogale, S. (2014). 3D micro-porous conducting carbon beehive by single step polymer carbonization for high performance supercapacitors: the magic of in situ porogen formation. Energy Environ. Sci. 7, 728-735.

Qian, B., Wang, G., Ling, Z., Dong, Q., Wu, T., Zhang, X., and Qiu, J. (2015). Sulfonated Graphene as Cation-Selective Coating: A New Strategy for High-Performance Membrane Capacitive Deionization. Adv. Mater. Interfaces 2, 1500372.

Qiu, H., Lv, L., Pan, B., Zhang, Q., Zhang, W., and Zhang, Q. (2009). Critical review in adsorption kinetic models. J. Zhejiang Univ. Sci. A 10, 716-724.

Rabe, M., Verdes, D., RankI, M., Artus, G.R.J., and Seeger, S. (2007). A Comprehensive Study of Concepts and Phenomena of the Nonspecific Adsorption of b-Lactoglobulin. ChemPhysChem 8, 862 872.

Rabe, M., Verdes, D., and Seeger, S. (2011). Understanding protein adsorption phenomena at solid surfaces. Adv. Colloid Interface Sci. 162, 87-106.

Rentsch, S., Siegenthaler, H., Papastavrou, G., April, R. V, Final, I., and June, F. (2007). Diffuse Layer Properties of Thiol-Modified Gold Electrodes Probed by Direct Force Measurements. Langmuir 23, 9083-9091. 
Ricci, M., Spijker, P., and Voitchovsky, K. (2014). Water-induced correlation between single ions imaged at the solid-liquid interface. Nat. Commun. 5, 1-8.

Roques-Carmes, T., Membrey, F., Filiâtre, C., and Foissy, a (2002). Potentiality of reflectometry for the study of the adsorption on dielectric and metal substrates: application to the adsorption of polyvinylimidazole on silica and gold. J. Colloid Interface Sci. 245, 257-266.

Roques-Carmes, T., Membrey, F., Kaisheva, M., Filiâtre, C., and Foissy, A. (2006). Reflectometric study of the adsorption of poly(vinyl imidazole) on a gold electrode, effects of $\mathrm{pH}$, and applied potential. J. Colloid Interface Sci. 299, 504-512.

Rose, M., Korenblit, Y., Kockrick, E., Borchardt, L., Oschatz, M., Kaskel, S., and Yushin, G. (2011). Hierarchical Micro- and Mesoporous Carbide-Derived Carbon as a High-Performance Electrode Material in Supercapacitors. Small 7, 1108-1117.

Rosen, M.A., Dincer, I., and Kanoglu, M. (2008). Role of exergy in increasing efficiency and sustainability and reducing environmental impact. Energy Policy 36, 128-137.

Rosso, M., Nguyen, A.T., Jong, E. De, Baggerman, J., Paulusse, J.M.J., Giesbers, M., Fokkink, R.G., Norde, W., Schroen, K., Rijn, C.J.M. Van, et al. (2011). Protein-Repellent Silicon Nitride Surfaces: UV-Induced Formation of Oligoethylene Oxide Monolayers. Appl. Mater. Interfaces 3, 697704.

Sahai, N. (2000). Estimating adsorption enthalpies and affinity sequences of monovalent electrolyte ions on oxide surfaces in aqueous solution. Geochim. Cosmochim. Acta 64, 3629-3641.

Sahin, M., Sahin, Y., and Ozcan, A. (2008). Ion chromatography-potentiometric detection of inorganic anions and cations using polypyrrole and overoxidized polypyrrole electrode. Sensors Actuators B 133, 5-14.

Salleh, F., Hassan, A., Yahya, R., Lafia-Araga, R.A., Azzahari, A.D., and Nazir, M.N.Z.M. (2014). Improvement in the mechanical performance and interfacial behavior of kenaf fiber reinforced high density polyethylene composites by the addition of maleic anhydride grafted high density polyethylene. J. Polym. Res. 21, 439.

Schaferling, M., and Bauerle, P. (2004). Porphyrin-functionalized oligo- and polythiophenes. J. Mater. Chem. 14, 1132-1141.

Scopes, R.K. (1994). Protein Purification (New York, NY: Springer New York).

Seader, J.D., Henley, E.J., and Roper, D.K. (1998). Separation Process Principles (John Wiley \& Sons, Inc.).

Seo, S., Jeon, H., Kwang, J., Kim, G., Park, D., Nojima, H., Lee, J., and Moon, S. (2010). Investigation on removal of hardness ions by capacitive deionization (CDI) for water softening applications. Water Res. 44, 2267-2275.

Shao, Y., Wang, J., Engelhard, M., Wang, C., and Lin, Y. (2010). Facile and controllable electrochemical reduction of graphene oxide and its applications. J. Mater. Chem. 20, 743-748. Simmons, N.J., Chin, K.O.A., Harnisch, J.A., Vaidya, B., Trahanovsky, W.S., Porter, M.D., and Angelici, R.J. (2000). Synthesis and characterization of a catechol-terminated alkanethiolate monolayer adsorbed on gold. J. Electroanal. Chem. 482, 178-187. 
Simon, G., Hanak, L., Grevlllot, G., Szanya, T., and Marton, G. (1996). Amino acid separation by preparative temperature-swing chromatography with flow reversal. J. Chromatogr. 732, 1-15.

Simon, R.A., Mallouk, T.E., Daube, K.A., and Wrighton, M.S. (1985). Electrochemical characterization of surface-bound redox polymers derived from 1,1'-bis[((3(triethoxysilyl)propyl)amino)carbonyl]cobaltocenium: charge transport, anion binding, and use in photoelectrochemical hydrogen generation. Inorg. Chem. 24, 3119-3126.

Singh, C., and Paul, A. (2015). Physisorbed hydroquinone on activated charcoal as a supercapacitor: An application of proton-coupled electron transfer. J. Phys. Chem. C 119, 11382-11390.

Song, B.H., and Palmore, G.T.R. (2006). Redox-Active Polypyrrole: Toward Polymer-Based Batteries. Adv. Mater. 18, 1764-1768.

Song, Y., Ye, G., Wang, Z., Kopeć, M., Xie, G., Yuan, R., Chen, J., Kowalewski, T., Wang, J., and Matyjaszewski, K. (2016a). Controlled Preparation of Well-Defined Mesoporous Carbon/Polymer Hybrids via Surface-Initiated ICAR ATRP with a High Dilution Strategy Assisted by Facile Polydopamine Chemistry. Macromolecules 49, 8943-8950.

Song, Y., Ye, G., Wu, F., Wang, Z., Liu, S., Kopec, M., Wang, Z., Chen, J., Wang, J., and Matyjaszewski, K. (2016b). Bioinspired Polydopamine (PDA) Chemistry Meets Ordered Mesoporous Carbons (OMCs): A Benign Surface Modification Strategy for Versatile Functionalization. Chem. Mater. 28, 5013-5021.

Spiegler, K.S., and EI-Sayed, Y.M. (2001). The energetics of desalination processes. Desalination 134, 109-128.

Srimuk, P., Kaasik, F., Kruner, B., Tolosa, A., Fleischmann, S., Jackel, N., Tekeli, M.C., Aslan, M., Suss, M.E., and Presser, V. (2016). MXene as a novel intercalation-type pseudocapacitive cathode and anode for capacitive deionization. J. Mater. Chem. A 4, 18265-18271.

Srimuk, P., Lee, J., Fleischmann, S., Choudhury, S., Jackel, N., Zeiger, M., Kim, C., Aslan, M., and Presser, V. (2017). Faradaic deionization of brackish and sea water via pseudocapacitive cation and anion intercalation into few-layered molybdenum disulfide. J. Mater. Chem. A 5, 15640-15649. Srinivasan, S., and Stoner, G. (1970). Adsorption of Blood Proteins on Metals Using Capacitance Techniques. J. Phys. Chem. 74, 1088-1094.

Stolberg, L., Richer, J., Lipkgwski, J., and Irish, D.E. (1986). Adsorption of pyridine at the polycrystalline gold-solution interface. J. Electronal. Chem. 207, 213-234.

Su, X., and Hatton, T.A. (2017a). Redox-electrodes for selective electrochemical separations. Adv. Colloid Interface Sci. 244, 6-20.

Su, X., and Hatton, T.A. (2017b). Electrosorption at functional interfaces: from molecular-level interactions to electrochemical cell design. Phys. Chem. Chem. Phys. 19, 23570-23584.

Su, X., Kulik, H.J., Jamison, T.F., and Hatton, T.A. (2016). Anion-Selective Redox Electrodes : Electrochemically Mediated Separation with Heterogeneous Organometallic Interfaces. Adv. Funct. Mater. 26, 3394-3404. 
Su, X., Hu, J., Kauke, M.J., Dalbosco, L., Thomas, J., Gonzalez, C.C., Zhu, E., Franzreb, M., Jamison, T.F., and Hatton, T.A. (2017a). Redox Interfaces for Electrochemically Controlled Protein Surface Interactions: Bioseparations and Heterogeneous Enzyme Catalysis. Chem. Mater. 29, 57025712.

Su, Y., Zhi, Z., Gao, Q., Xie, M., Yu, M., Lei, B., Li, P., and Ma, P.X. (2017b). Autoclaving-Derived Surface Coating with In Vitro and In Vivo Antimicrobial and Antibiofilm Efficacies. Adv. Healthc. Mater. 6, 1601173.

Suarez-Herrera, M.F., and Feliu, J.M. (2009). Electrochemical Properties of Thin Films of Polythiophene Polymerized on Basal Plane Platinum Electrodes in Nonaqueous Media. J. Phys. Chem. B 113, 1899-1905.

Sulaymon, A. H.Abbar, A.H. (2012). Scale-Up of Electrochemical Reactors. In Electrolysis, (InTech), pp. 189-202.

Suss, M.E., and Presser, V. (2018). Water Desalination with Energy Storage Electrode Materials. Joule 2, 10-15.

Suss, M.E., Baumann, T.F., Bourcier, W.L., Spadaccini, C.M., Rose, K.A., Santiago, J.G., and Stadermann, M. (2012). Capacitive desalination with flow-through electrodes. Energy Environ. Sci. 5, 9511.

Suss, M.E., Baumann, T.F., Worsley, M.A., Rose, K.A., Jaramillo, T.F., Stadermann, M., and Santiago, J.G. (2013). Impedance-based study of capacitive porous carbon electrodes with hierarchical and bimodal porosity. J. Power Sources 241, 266-273.

Suss, M.E., Porada, S., Sun, X., Biesheuvel, P.M., Yoon, J., and Presser, V. (2015). Water desalination via capacitive deionization: what is it and what can we expect from it? Energy Environ. Sci. 8, 2296-2319.

Szargut, J. (1989). Chemical Exergies of the Elements. Appl. Energy 32, 269-286.

Takada, T., Baba, T., and Abe, S. (2016). Simple Process for Sidewall Modification of Chain Radicals Generated by Ultraviolet-Induced C - Cl Bond Dissociation of Polystyrene Derivatives. C 2, 20.

Tamer, U., Yates, B., Galal, A., Gbatu, T., LaRue, R., Schmiesing, C., Temsamani, K., Ceylan, O., and Mark Jr, H.B. (2003). Electrochemically Aided Control of Solid Phase Micro-Extraction (EASPME) Using Conducting Polymer-Coated Solid Substrates Applicable to Neutral Analytes. Microchim. Acta 143, 205-215.

Tamime, A.Y. (2008). Milk Processing and Quality Management (Oxford, UK: Wiley-Blackwell).

Tang, W., Liang, J., He, D., Gong, J., Tang, L., Liu, Z., Wang, D., and Zeng, G. (2019). Various cell architectures of capacitive deionization: Recent advances and future trends. Water Res. 150, 225251.

Tarhan, Ö., and Harsa, Ş. (2014). Nanotubular structures developed from whey-based a-lactalbumin fractions for food applications. Biotechnol. Prog. 30, 1301-1310.

Teasdale, P.R., and Walfacet, G.G. (1993). Molecular Recognition Using Conducting Polymers: Basis of an Electrochemical Sensing Technology. Analyst 118, 329-334. 
Terefe, N.S., Glagovskaia, O., De Silva, K., and Stockmann, R. (2014). Application of stimuli responsive polymers for sustainable ion exchange chromatography. Food Bioprod. Process. 92, 208225.

Trócoli, R., Battistel, A., and Mantia, F. La (2015). Nickel Hexacyanoferrate as Suitable Alternative to Ag for Electrochemical Lithium Recovery. ChemSusChem 8, 2514-2519.

Ugur, T., Yucel, S., Nusret, E., Yasemin, U., Kadir, P., and Attila, Y. (2004). Preparation of sulfonated overoxidized polypyrrole film applicable as an SPME tool for cationic analytes. J.

Electroanal. Chem. 570, 6-12.

United Nation (2017). Sustainable Development Goal 6.

United Nations (2016). 2016 United Nations World Water Development Report, Water and Jobs.

Velasco-Velez, J.-J., Wu, C.H., Pascal, T.A., Wan, L.F., Guo, J., Prendergast, D., and Salmeron, M. (2014). The structure of interfacial water on gold electrodes studied by $x$-ray absorption spectroscopy. Science (80-. ). 346, 831-834.

Vijh, A.K., and Conway, B.E. (1967). Electrode Kinetic Aspects of the Kolbe Reaction. Chem. Rev. 67, 623-664.

Vlachova, J., Labuda, J., Hynek, D., Zitka, O., and Kizek, R. (2015). Utilization of graphene oxide electrophoretic deposition for construction of electrochemical sensors and biosensors. J. Met.

Nanotechnologies 3, 57-63.

Vogler, E.A. (2012). Protein adsorption in three dimensions. Biomaterials 33, 1201-1237.

Vorotyntsev, M.A., Vieil, E., and Heinze, J. (1998). Charging process in polypyrrole films: effect of ion association. J. Electroanal. Chem. 450, 121-141.

Walsh, G. (2002). Proteins: Biochemistry and Biotechnology (John Wiley \& Sons, Ltd.).

Walsh, F.C., and Ponce de León, C. (2018). Progress in electrochemical flow reactors for laboratory and pilot scale processing. Electrochim. Acta 280, 121-148.

Wang, H., Zhang, D., Yan, T., Wen, X., Zhang, J., Shi, L., and Zhong, Q. (2013). Three-dimensional macroporous graphene architectures as high performance electrodes for capacitive deionization. J. Mater. Chem. A 1, 11778.

Wang, H., Yan, T., Liu, P., Chen, G., Shi, L., Zhang, J., Zhong, Q., and Zhang, D. (2016). In situ creating interconnected pores across $3 \mathrm{D}$ graphene architectures and their application as high performance electrodes for flow-through deionization capacitors. J. Mater. Chem. A 4, 4908-4919. Wang, J., Rivas, G., Jiang, M., and Zhang, X. (1999). Electrochemically Induced Release of DNA from Gold Ultramicroelectrodes. Langmuir 15, 6541-6545.

Wang, J., Liang, M., Fang, Y., Qiu, T., Zhang, J., and Zhi, L. (2012). Rod-coating: Towards largearea fabrication of uniform reduced graphene oxide films for flexible touch screens. Adv. Mater. 24 , 2874-2878.

Wang, J., Ge, X., Liu, Z., Thia, L., Yan, Y., Xiao, W., and Wang, X. (2017). Heterogeneous Electrocatalyst with Molecular Cobalt lons Serving as the Center of Active Sites. J. Am. Chem. Soc. 139, 1878-1884. 
Wang, P., Meng, J., Xu, M., Yuan, T., Yang, N., Sun, T., Zhang, Y., Feng, X., and Cheng, B. (2015a). A simple but efficient zwitterionization method towards cellulose membrane with superior antifouling property and biocompatibility. J. Memb. Sci. 492, 547-558.

Wang, Y., El-deen, A.G., Li, P., Oh, B.H.L., Guo, Z., Khin, M.M., Vikhe, Y.S., Wang, J., Hu, R.G., Boom, R.M., et al. (2015b). High-Performance Capacitive Deionization Disinfection of Water with Graphene Oxide- graft - Electrode Coating. ACS Nano 9, 10142-10157.

Wang, Z., Feng, Y., Hao, X., Huang, W., and Feng, X. (2014a). A novel potential-responsive ion exchange film system for heavy metal removal. J. Mater. Chem. A 2, 10263-10272.

Wang, Z., Ma, Y., Hao, X., Huang, W., Guan, G., and Abudula, A. (2014b). Enhancement of heavy metals removal efficiency from liquid wastes by using potential-triggered proton self-exchange effects. Electrochim. Acta 130, 40-45.

Watcharotone, S., Dikin, D. a, Stankovich, S., Piner, R., Jung, I., Dommett, G.H.B., Evmenenko, G., Wu, S.E., Chen, S.F., Liu, C.P., et al. (2007). Graphene- Silica Composite Thin Films as Transparent Conductors. Nano Lett. 7, 1888-1892.

Welgemoed, T.J., and Schutte, C.F. (2005). Capacitive Deionization Technology: An alternative desalination solution. Desalination 183, 327-340.

Wesselingh, J.A., Vonk, P., and Kraaijeveld, G. (1995). Exploring the Maxwell-Stefan description of ion exchange. Chem. Eng. J. 57, 75-89.

White, N., Misovich, M., Alemayehu, E., Yaroshchuk, A., and Bruening, M.L. (2016). Highly selective separations of multivalent and monovalent cations in electrodialysis through Nafion membranes coated with polyelectrolyte multilayers. Polymer (Guildf). 103, 478-485.

Wöbkenberg, P.H., Eda, G., Leem, D.-S., de Mello, J.C., Bradley, D.D.C., Chhowalla, M., and Anthopoulos, T.D. (2011). Reduced Graphene Oxide Electrodes for Large Area Organic Electronics. Adv. Mater. 23, 1558-1562.

Woo, S., Dokko, K., Nakano, H., and Kanamura, K. (2008). Preparation of three dimensionally ordered macroporous carbon with mesoporous walls for electric double-layer capacitors. J. Mater. Chem. 18, 1674-1680.

World Economic Forum (2017). The Global Risks Report 2017.

Wu, J., and Pawliszyn, J. (2001). Preparation and applications of polypyrrole films in solid-phase microextraction. J. Chromatogr. A 909, 37-52.

Wu, B., Mathews, N., and Sum, T.-C. (2017). Surface Plasmon Resonance. In Plasmonic Organic Solar Cells, Springer, ed. pp. 25-31.

Wu, J., Mullett, W.M., and Pawliszyn, J. (2002). Electrochemically Controlled Solid-Phase Microextraction Based on Conductive Polypyrrole Films. Anal. Chem. 74, 4855-4859.

Wu, T., Wang, G., Dong, Q., Qian, B., Meng, Y., and Qiu, J. (2015). Asymmetric capacitive deionization utilizing nitric acid treated activated carbon fiber as the cathode. Electrochim. Acta 176, 426-433.

Wu, T., Wang, G., Zhan, F., Dong, Q., Ren, Q., Wang, J., and Qiu, J. (2016a). Surface-treated carbon electrodes with modified potential of zero charge for capacitive deionization. Water Res. 93, 30-37. 
Wu, T., Wang, G., Zhan, F., Dong, Q., Ren, Q., Wang, J., and Qiu, J. (2016b). Surface-treated carbon electrodes with modified potential of zero charge for capacitive deionization. Water Res. 93, 30-37.

Xie, Y., Liao, C., and Zhou, J. (2013). Effects of external electric fields on lysozyme adsorption by molecular dynamics simulations. Biophys. Chem. 179, 26-34.

Xie, Y., Pan, Y., Zhang, R., Liang, Y., and Li, Z. (2015). Modulating protein behaviors on responsive surface by external electric fields: A molecular dynamics study. Appl. Surf. Sci. 326, 55-65.

Xing, F., Li, T., Li, J., Zhu, H., Wang, N., and Cao, X. (2017). Nano Energy Chemically exfoliated MoS2 for capacitive deionization of saline water. Nano Energy 31, 590-595.

Xu, B., Zhang, L., Pei, Y.T., Luo, J.K., Tao, S.W., De Hosson, J.T.M., and Fu, Y.Q. (2012). ElectroResponsive Polystyrene Shape Memory Polymer Nanocomposites. Nanosci. Nanotechnol. Lett. 4, 17.

Yamini, Y., Seidi, S., and Rezazadeh, M. (2014). Electrical field-induced extraction and separation techniques: Promising trends in analytical chemistry - A review. Anal. Chim. Acta 814, 1-22.

Yang, J., Zou, L., and Roy, N. (2013). Ion-selective carbon nanotube electrodes in capacitive deionisation. Electrochim. Acta 91, 11-19.

Yang, L., Kong, J., Yee, W.A., Liu, W., Phua, S.L., Toh, C.L., Huang, S., and Lu, X. (2012). Highly conductive graphene by low-temperature thermal reduction and in situ preparation of conductive polymer nanocomposites. Nanoscale 4, 4968.

Yang, Z., Jin, L., Lu, G., Xiao, Q., Zhang, Y., Jing, L., Zhang, X.-X., Yan, Y.-M., and Sun, K.-N. (2014). Sponge-Templated Preparation of High Surface Area Graphene with Ultrahigh Capacitive Deionization Performance. Adv. Funct. Mater. 24, 3917-3925.

Yates, B.J., Temsamani, K.R., Ceylan, O., Oztemiz, S., Gbatu, T.P., LaRue, R.A., Tamer, U., and Mark, H.. (2002). Electrochemical control of solid phase micro-extraction: conducting polymer coated film material applicable for preconcentration/analysis of neutral species. Talanta 58, 739-745.

Yeo, J., and Choi, J. (2013). Enhancement of nitrate removal from a solution of mixed nitrate, chloride and sulfate ions using a nitrate-selective carbon electrode. Desalination 320, 10-16.

Yeo, W., Hodneland, C.D., and Mrksich, M. (2001). Electroactive Monolayer Substrates that Selectively Release Adherent Cells. Chembiochem 7, 590-593.

Yeo, W., Yousaf, M.N., and Mrksich, M. (2003). Dynamic Interfaces between Cells and Surfaces: Electroactive Substrates that Sequentially Release and Attach Cells. J. Am. Chem. Soc. 125, 1499414995.

Yeung, B.C.L., Iqbal, P., Allan, M., Lashkor, M., Preece, J.A., and Mendes, P.M. (2010). Tuning Specific Biomolecular Interactions Using Electro-Switchable Oligopeptide Surfaces. Adv. Funct. Mater. 20, 2657-2663.

Yin, Z., Wu, S., Zhou, X., Huang, X., Zhang, Q., Boey, F., and Zhang, H. (2010). Electrochemical deposition of $\mathrm{ZnO}$ nanorods on transparent reduced graphene oxide electrodes for hybrid solar cells. Small 6, 307-312.

Yin, Z., Zhu, J., He, Q., Cao, X., Tan, C., Chen, H., Yan, Q., and Zhang, H. (2014). Graphene-Based materials for solar cell applications. Adv. Energy Mater. 4, 1-19. 
Yoon, H., Lee, J., Kim, S., and Yoon, J. (2018). Hydrometallurgy Electrochemical sodium ion impurity removal system for producing high purity $\mathrm{KCl}$. Hydrometallurgy $175,354-358$.

Yoon, H., Lee, J., Kim, S., and Yoon, J. (2019). Review of concepts and applications of electrochemical ion separation (EIONS) process. Sep. Purif. Technol. 215, 190-207.

Yousaf, M.N., Houseman, B.T., and Mrksich, M. (2001a). Turning On Cell Migration with Electroactive Substrates. Angew. Chem. Int. Ed. 40, 1093-1096.

Yousaf, M.N., Houseman, B.T., and Mrksich, M. (2001b). Using electroactive substrates to pattern the attachment of two different cell populations. Proc. Natl. Acad. Sci. 98, 5992-5996.

Yu, G., Hu, L., Vosgueritchian, M., Wang, H., Xie, X., McDonough, J.R., Cui, X., Cui, Y., and Bao, Z. (2011). Solution-processed graphene/MnO2nanostructured textiles for high-performance electrochemical capacitors. Nano Lett. 11, 2905-2911.

Yu, H., He, J., Sun, L., Tanaka, S., and Fugetsu, B. (2013). Influence of the electrochemical reduction process on the performance of graphene-based capacitors. Carbon N. Y. 51, 94-101.

Zhai, S., Ma, Y., Chen, Y., Li, D., Cao, J., Liu, Y., Cai, M., Xie, X., Chen, Y., and Luo, X. (2014). Synthesis of an amphiphilic block copolymer containing zwitterionic sulfobetaine as a novel $\mathrm{pH}-$ sensitive drug carrier. Polym. Chem. 5, 1285-1297.

Zhang, X.G. (2001). Electrochemistry of Silicon and Its Oxide (Kluwer Academic Publishers).

Zhang, C., He, D., Ma, J., Tang, W., and Waite, T.D. (2018a). Faradaic reactions in capacitive deionization (CDI) - problems and possibilities : A review. Water Res. 128, 314-330.

Zhang, P., Fritz, P.A., Schroën, K., Duan, H., Boom, R.M., and Chan-Park, M.B. (2018b).

Zwitterionic Polymer Modified Porous Carbon for High-Performance and Antifouling Capacitive Desalination. Appl. Mater. Interfaces 10, 33564-33573.

Zhang, X., Zhang, D., Chen, Y., Sun, X., and Ma, Y. (2012). Electrochemical reduction of graphene oxide films: Preparation, characterization and their electrochemical properties. Chinese Sci. Bull. 57, 3045-3050.

Zhao, C., Liu, G., Sun, N., Zhang, X., Wang, G., Zhang, Y., Zhanga, H., and Zhao, H. (2018).

Biomass-derived $\mathrm{N}$-doped porous carbon as electrode materials for $\mathrm{Zn}$-air battery powered capacitive deionization. Chem. Eng. J. 334, 1270-1280.

Zhao, R., Biesheuvel, P.M., and Wal, A. Van Der (2012a). Energy consumption and constant current operation in membrane capacitive deionization. Energy Environ. Sci. 5, 9520.

Zhao, R., Soestbergen, M. Van, Rijnaarts, H.H.M., Wal, A. Van Der, Bazant, M.Z., and Biesheuvel, P.M. (2012b). Time-dependent ion selectivity in capacitive charging of porous electrodes. J. Colloid Interface Sci. 384, 38-44.

Zhao, Y., Zhang, P., Sun, J., Liu, C., Yi, Z., Zhu, L., and Xu, Y. (2015). Versatile antifouling polyethersulfone filtration membranes modified via surface grafting of zwitterionic polymers from a reactive amphiphilic copolymer additive. J. Colloid Interface Sci. 448, 380-388.

Zheng, F., Yang, Y., and Chen, Q. (2014). High lithium anodic performance of highly nitrogen-doped porous carbon prepared from a metal-organic framework. Nat. Commun. 5, 1-10. 
Zhou, A., and Xie, N. (1999). Investigation of Electrosorption of Organic Molecules onto Gold and Nickel Electrodes Using an Electrochemical Quartz Crystal Microbalance. J. Colloid Interface Sci. 220, 281-287.

Zhou, A., Xie, Q., Wu, Y., Cai, Y., Nie, L., and Yao, S. (2000). Study of the Adsorption of Glutathione on a Gold Electrode by Using Electrochemical Quartz Crystal Impedance, Electrochemical Impedance Spectroscopy, and Cyclic Voltammetry. J. Colloid Interface Sci. 229, 12-20.

Zhu, M., Lan, J., Zhang, X., Sui, G., and Yang, X. (2017). Porous carbon derived from Ailanthus altissima with unique honeycomb-like microstructure for high-performance supercapacitors. New $\mathrm{J}$. Chem. 41, 4281-4285.

Zotti, G., Zecchin, S., Schiavon, G., Berlin, A., Pagani, G., and Canavesi, A. (1997). DopingInduced lon-Exchange in the Highly Conjugated Self-Doped Polythiophene from Anodic Coupling of 4(4H-Cyclopentadithien-4-yl)butanesulfonate. Chem. Mater. 9, 2940-2944. 

MISCELANEOUS 


\section{SUMMARY}

Separation process are at the heart of many industries ranging from chemical to medical and food. To create innovative products, first specific components need to be purified or fractionated from the source of origin. Generally, this requires a large amount of energy and chemicals; therefore, more sustainable processing options need to be designed. Within this thesis, we investigate electrochemical separation processes for ions and proteins. These processes bear great potential to reduce energy and solvent use compared to membrane or chromatographic techniques, since electric energy is directly used without further conversion, and fast and easy switching between e.g. negative and positive surfaces makes it unnecessary to regenerate solid supports with solvents or buffers. Nevertheless, to unfold the full potential of electrochemical separation processes, development of electrodes for selective separation, and the design of the overall process need to be taken to a next level. Both issues are crucial when extending the principles about ion separation to other molecules such as proteins that to date have been hardly explored in this context.

We first review in Chapter 2 the current state of the art of electrochemical separation processes using capacitive or faradaic principles applied to small ions, proteins, and cells. An important point that is reviewed is the difference between capacitive deionization and inverted capacitive deionization. In the former process, ions are stored capacitively in the electric double layer when a constant electrode potential or current is applied. The release of the ions occurs when releasing the electric bias or by reversing it. In contrast during the latter process, ions are stored due to chemical surface charges and the additional electric potential is only applied during the regeneration phase. Since in this option selective surface coatings may be used, it allows for specifically targeting molecules within a mixture, and still operating without solvents for regeneration. This is a major advantage for protein separation, and thus used in the following chapters, starting from a system with salt that later becomes more complex by the introduction of protein.

In Chapter $\mathbf{3}$ and $\mathbf{4}$ we investigate simple and modified activated carbon electrodes in an inverted capacitive deionization process. We found that ion adsorption at $0 \mathrm{~V}$ was possible for simple activated carbon electrodes when used with ion 
exchange membranes in front of the electrodes (inverted capacitive membrane deionization). We also found a similar separation pattern for electrodes with polyelectrolytes added to the matrix, which in combination with ion exchange membranes resulted in a process that is competitive with conventional capacitive deionization at very low exergy loss. When working with a solution that contains proteins, electrode fouling is a challenge. In Chapter $\mathbf{5}$ we show that the application of hierarchical carbon electrodes coated with a zwitterionic polymer brush avoids protein adsorption and increases the life time of the electrodes during desalination. However, when proteins need to be separated, they need to interact with the surface of the electrode while still desorbing upon an electric trigger.

In Chapter $\mathbf{6}$ we show that activated carbon electrodes containing cationic and anionic polyelectrolytes have the ability to reversibly adsorb and release protein by applying an electric potential bias. While proteins were adsorbed, salt was desorbed and vice versa, therewith also showing a potential for desalting protein solutions. In Chapter 7 we investigated the forces acting on salt and proteins in detail, and measured an increase in electric and hydration repulsion at the electrode interface due to an externally applied electric potential which influenced surface wetting. Overall, we concluded that the changes induced by the electric potential were sufficient to influence protein ad- and desorption. Since in Chapter 7 the measurements were conducted on gold substrates, we presented in Chapter 8 as a first step toward carbon based electrodes reduced graphene oxide coated silicon substrates to study protein ad- and desorption.

The findings described in this thesis are important for the development of novel electrochemical separation processes for complex molecules, and they lay the ground work for a next generation of sustainable separation technologies. Thus, in Chapter 9 our findings were put into perspective, and we highlighted research required to further design electrochemical separation processes. We indicated that suitable (responsive) surface coatings, and optimized process designs for large scale applications are key to unlock the full potential of electrochemical separation processes and to guarantee their successful implementation. 


\section{ACKNOWLEDGMENT}

The last four years have passed so quickly that it seems unreal to start writing the acknowledgment. They have been informative, exciting and for sure truly inspiring. For an incredible supervision and mentorship I would like to thank Karin. You slowed me down when I got overexcited and motivated me when being insecure, always being honest and direct. I truly appreciate all the time and effort you put into our weekly meetings, reviewing my publications and discussing social skills and the future. You helped me to identify strengths and weaknesses so that I am now ready for my next step. I would also like to thank Remko for his trust and support throughout my PhD, challenging me scientifically an motivating me with honest feedback. Next to that I also very much appreciate the input Prof. Mary gave me, when staying at Nanyang Technological University in Singapore. This allowed me to learn a lot about polymer synthesis and surface modifications as well as cultural differences between Europe and Singapore. In the same context I would like to thank Penghui, for being curious, positive and engaged in our shared projects.

Thank you also, to my project partners from the Institute for Sustainable Process Technology (ISPT), Peter de Jong, Hilde Wijngaard, Guilherme Ferreira, Albert van der Padt, Edwin Poiesz and Wil Duivenvoorden, always making the cluster meetings interesting and a good source for inspirations. At the same time I would like to thank Eric Karruppannan, Johan Belgraver and Hans Meijer for excellent creativity and support at the workshop as well as Han Zuilhof, Mieke Kleijn and Louis de Smet for additional scientific advice within Wageningen University. Also the chats with Jouke Dykstra and Maarten Biesheuvel wherever we met on the globe helped me to develop and improve my scientific understanding of electrochemical separation processes and made me appreciate the positive atmosphere in the CDI community.

Furthermore, Bijoy you really arrived at FPE at the right moment and I would like to thank you a lot for your help and patience in scientific and Dutch matters. Maurice and Jos you have been an incredible help with installing new devices in the lab and challenging, thus improving, my experimental set ups. Thanks also to all my students who helped me to realize and further develop my ideas: Robert, Loes, Hilde, Saskia, 
Laurens, Sander, Nikolin, Koen, Ivy, Tom and Jelle. I learned as much from you as hopefully vice versa.

A big thank you as well to all my colleagues and friends at FPE for making all the coffee breaks, lunches and social events fun. Especially, I would like to thank Juliana, Patricia, Fiona, Eline, Isabel, Eric, Elvira, Konstantina, Anton, Victor, Dimitri, Victor, Qinhui, Lu, $\mathrm{Yu}$, Izabella, and Anouk for continuously smiling and laughing along side of me at work, or during numerous international dinners. Andrea and Anna, you are amazing colleagues, housemates and friends. Without you Wageningen wouldn't have been the same.

I also would like to thank my family for their support, Mama, Papa, Ann-Kristina, Pipo without you I wouldn't have reached this far. The same counts for the support from Mahler, little Frederik, Georg, Gertraud, and all my friends around the world, thank you for believing in me. Last but not least my biggest source of motivation, energy and compassion. Alex, thanks for being at my side no matter what. 


\section{ABOUT THE AUTHOR}

Pina Fritz was born on October $26^{\text {th }} 1989$ in Paderborn (Germany). After graduating from the European School of Karlsruhe (Germany) in 2008, obtaining her European baccalaureate, she moved to Zurich (Switzerland) to study Food Science at the Swiss Federal Institute of Technology. In 2012 she completed her bachelor's degree and in 2015 her master's degree. In between she went for an exchange semester to the National University of

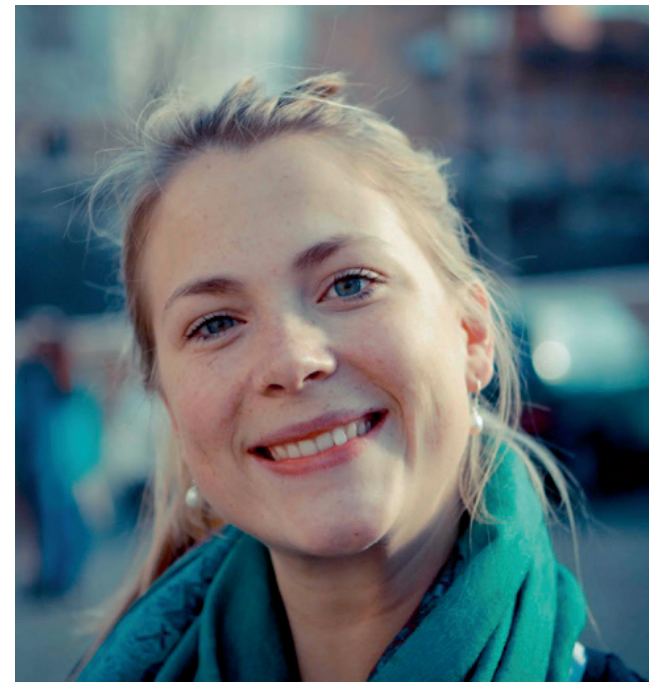
Singapore. Her bachelor thesis focused on the genetic modification of rice to increase the iron content in the grain and her master thesis was about the rheological behavior of polymer suspensions during a 3D printing process. After graduating from her master's program, she spent 7 months at Harvard University (USA) working on skeletal muscle tissue engineering, followed by a junior scientist position at Food Concept and Physical Design in Flüh (Switzerland). In 2015, Pina started her joint-PhD program at Wageningen University (The Netherlands) and Nanyang Technological University (Singapore). During her research she focused on the development of electrochemical separation processes for ions and proteins. This included the design of suitable polyelectrolyte-carbon electrodes, and studying their separation performance as well as defining suitable exergy indicators. Next to this she also studied in great detail the underlying surface forces impacting protein ad- and desorption to and from electrode surfaces depending on the applied potential.

Contact: pina.fritz@gmail.com 


\section{PUBLICATIONS}

P.A. Fritz, F.K. Zisopoulos, S. Verheggen, K. Schroën, R.M. Boom, Exergy analysis of membrane capacitive deionization (MCDI), Desalination. 444 (2018) 162-168.

P. Zhang, P.A. Fritz, K. Schroën, H. Duan, R.M. Boom, M.B. Chan-Park, Zwitterionic Polymer Modified Porous Carbon for High-Performance and Antifouling Capacitive Desalination, Appl. Mater. Interfaces. 10 (2018) 33564-33573.

P.A. Fritz, S.C. Lange, M. Giesbers, H. Zuilhof, R.M. Boom, K. Schroën, Simultaneous Silicon Oxide Growth and Electrophoretic Deposition of Graphene Oxide, Langmuir. 35 (2019) 3717-3723.

P.A. Fritz, R.M. Boom, K. Schroën, Polyelectrolyte-activated carbon composite electrodes for inverted membrane capacitive deionization (iMCDI), Sep. Purif. Technol. 220 (2019) 145-151.

P.A. Fritz, T. Bruschinski, S. Sahin, P. Zhang, L. Smet, M. Chan-Park, R. Boom, K. Schroën, Steering protein and salt ad- and desorption by an electrical switch applied to tailor made electrodes. Submitted.

P.A. Fritz, B. Bera, J. Berg, I. Visser, M. Kleijn, R. Boom, K. Schroën, Controlling electrostatic and hydration interactions electrochemically for protein adsorption and desorption. Submitted.

P.A. Fritz, R. Boom, K. Schroën, Electrochemically driven adsorptive separation techniques: from ions to proteins and cells in liquid streams 


\section{TRAINING ACTIVITIES}

\begin{tabular}{|c|c|c|}
\hline Course name & Organizing institute (s) & Year \\
\hline \multicolumn{3}{|l|}{ Discipline specific courses } \\
\hline Objective molecular affinity separations & OSPT & 2016 \\
\hline Advanced thermodynamics & OSPT & 2016 \\
\hline $\begin{array}{l}\text { International school on modeling and simulation in } \\
\text { food and bio processes }\end{array}$ & ISEKI-Food Association & 2016 \\
\hline Process economics \& cost engineering & OSPT & 2016 \\
\hline Numerical methods for chemical engineers & OSPT & 2016 \\
\hline Ameland summer school: smart materials & 4TU & 2017 \\
\hline Sustainability analysis in food biobased production & VLAG & 2016 \\
\hline Graphene and 2-dimensional materials & $\begin{array}{l}\text { Coursera (Manchester } \\
\text { University) }\end{array}$ & 2016 \\
\hline $\begin{array}{l}\text { Elektrochemie für naturwissenschaftler, ingenieure } \\
\text { und techniker }\end{array}$ & $\begin{array}{l}\text { DECHEMA } \\
\text { Forschungsinsitut }\end{array}$ & 2016 \\
\hline Han-sur-lesse winter school & WUR & 2018 \\
\hline \multicolumn{3}{|l|}{ General courses } \\
\hline PhD competence assessment & WGS & 2016 \\
\hline PhD week & VLAG & 2016 \\
\hline Teaching an supervising thesis students & WGS & 2016 \\
\hline Teaching an online course & $\begin{array}{l}\text { Educational staff } \\
\text { development }\end{array}$ & 2016 \\
\hline Brain training & WGS & 2018 \\
\hline Adobe & WGS & 2018 \\
\hline Debate training & WGS & 2018 \\
\hline Career Perspectives & WGS & 2019 \\
\hline \multicolumn{3}{|l|}{ Optional } \\
\hline Preparation of research proposal & FPE & 2016 \\
\hline Weekly group meetings & FPE & 2015-2019 \\
\hline PhD excursion (Switzerland, Germany) & FPE & 2016 \\
\hline PhD excursion (Canada) & FPE & 2018 \\
\hline
\end{tabular}




\section{LIST OF CONFERENCES}

\begin{tabular}{lllc}
\hline Conferences & Oral/Poster & Title & Year \\
\hline $\begin{array}{l}\text { CDI\&E 2017 } \\
\text { (Seoul, Korea) }\end{array}$ & Oral presentation & $\begin{array}{l}\text { Exergy analysis for } \\
\text { capacitive deionization (CDI) }\end{array}$ & 2017 \\
\hline $\begin{array}{l}\text { EIS conference } \\
\text { (Bologna, Italy) }\end{array}$ & Oral presentation & $\begin{array}{l}\text { Simultaneous Silicon Oxide Growth and } \\
\text { Electrophoretic Deposition of Graphene } \\
\text { Oxide }\end{array}$ & 2018 \\
\hline $\begin{array}{l}\text { CDI\&E 2019 } \\
\text { (Beijing, China) }\end{array}$ & Oral presentation & $\begin{array}{l}\text { Polyelectrolyte-activated carbon } \\
\text { composite electrodes for } \\
\text { inverted (membrane) capacitive deionization }\end{array}$ & Inverted capacitive protein separation \\
\hline $\begin{array}{l}\text { ACS conference } \\
\text { (San Diego, USA) }\end{array}$ & Oral presentation & $\begin{array}{l}\text { Capacitive protein separation: from } \\
\text { electrode preparation to process design } \\
\text { that also affects salt removal }\end{array}$ & 2019 \\
\hline
\end{tabular}

\section{TEACHING}

Laboratory class of enzymology $(2017,2018,2019)$

5 BSc students

6 MSc students 
The research described in this thesis was supported financially by the Institute for Sustainable Process Technology (ISPT)

Printed by Digiforce - Proefschriftmaken.nl 